\title{
Singlet Oxygen Quantum Yields in Environmental Waters
}

\section{Review Article}

Author(s):

Ossola, Rachele (D); Jönsson, Oskar M.; Moor, Kyle; McNeill, Kristopher (D)

Publication date:

2021-04-14

Permanent link:

https://doi.org/10.3929/ethz-b-000478666

\section{Rights / license:}

Creative Commons Attribution 4.0 International

Originally published in:

Chemical Reviews 121(7), https://doi.org/10.1021/acs.chemrev.0c00781

\section{Funding acknowledgement:}

188565 - Reactivity-based characterization of photoexcited natural organic matter (SNF) 


\title{
Singlet Oxygen Quantum Yields in Environmental Waters
}

\author{
Rachele Ossola, Oskar Martin Jönsson, Kyle Moor, and Kristopher McNeill*
}

Cite This: Chem. Rev. 2021, 121, 4100-4146

Read Online

\section{ACCESS | Lلll Metrics \& More | 回 Article Recommendations | S1 Supporting Information}

ABSTRACT: Singlet oxygen $\left({ }^{1} \mathrm{O}_{2}\right)$ is a reactive oxygen species produced in sunlit waters via energy transfer from the triplet states of natural sensitizers. There has been an increasing interest in measuring apparent ${ }^{1} \mathrm{O}_{2}$ quantum yields $\left(\Phi_{\Delta}\right)$ of aquatic and atmospheric organic matter samples, driven in part by the fact that this parameter can be used for environmental fate modeling of organic contaminants and to advance our understanding of dissolved organic matter photophysics. However, the lack of reproducibility across research groups and publications remains a challenge that significantly limits the usability of literature data. In the first part of this review, we critically evaluate the experimental techniques that have been used to determine $\Phi_{\Delta}$ values of natural organic matter, we identify and quantify sources of errors that potentially explain the large variability in the

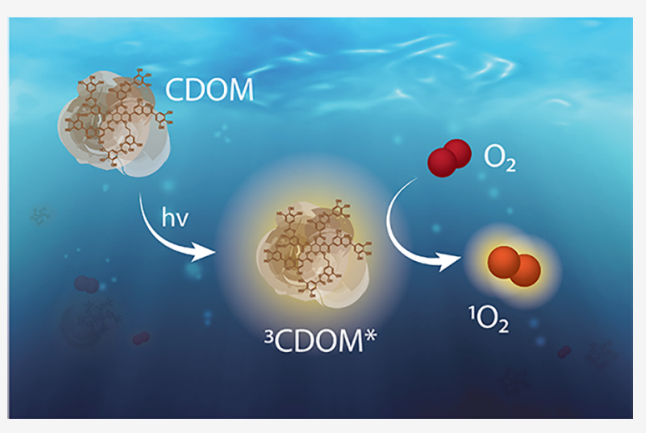
literature, and we provide general experimental recommendations for future studies. In the second part, we provide a qualitative overview of known $\Phi_{\Delta}$ trends as a function of organic matter type, isolation and extraction procedures, bulk water chemistry parameters, molecular and spectroscopic organic matter features, chemical treatments, wavelength, season, and location. This review is supplemented with a comprehensive database of $\Phi_{\Delta}$ values of environmental samples.

\section{CONTENTS}

1. Introduction

2. Brief Overview of Surface Water ${ }^{1} \mathrm{O}_{2}$ Photophysics

3. Methods for $\Phi_{\Delta}$ Determination in Environmental Systems

3.1. Derivation of the Basic Equations

3.1.1. Chemical Probe Methods

3.1.2. ${ }^{1} \mathrm{O}_{2}$ Phosphorescence Methods

3.2. Critical Discussion of the Parameters

3.2.1. Measuring Light Absorption

3.2.2. Measuring ${ }^{1} \mathrm{O}_{2}$ Production

3.3. Error Analysis

3.4. Experimental Recommendations and Research Gaps

4. Singlet Oxygen Quantum Yields in Environmentally Relevant Samples

4.1. Structure of the Database

4.2. DOM Types

4.2.1. Freshwater DOM

4.2.2. Wastewater DOM

4.2.3. Soil Organic Matter

4.2.4. Marine DOM

4.2.5. Atmospheric Organic Matter

4.2.6. Other Organic Matter Samples

4.3. Isolation and Extraction Procedures

4.3.1. Aquatic and Soil Samples

4.3.2. Atmospheric Samples

4.4. Short Summary and Research Gaps

5. Trends and Interpretation of Results

5.1. Water Chemistry

5.2. Molecular, Optical, and Photophysical DOM Features

4103

4104

4105

4105

4106

4107

4107

4112

4116

4119

4121

4122

4122

4122

4123

4124

4124

4124

4125

4125

4125

4127

4127

5.2.1. Macroscopic and Microscopic Molecular Features

5.2.2. Optical Features

5.2.3. Photophysical Features

5.3. Chemical Treatments

5.3.1. Reductive Treatments

5.3.2. Oxidative Treatments

5.4. Wavelength Dependence

5.5. Seasonal and Spatial Variability

5.5.1. Freshwater to Saltwater Systems

5.5.2. Lake Superior

5.6. Short Summary and Research Gaps

6. Final Remarks

Associated Content

Supporting Information

Author Information

Corresponding Author

Authors

Received: July 24, 2020

Published: March 8, 2021
4128

4128

4130

4130

4131

4133

4133

4133

4133

4134

4135

4135

4136

4136

4137

4138

4138

4138

4138

4138 


\section{Notes}

Biographies

Acknowledgments

Abbreviations list

References

\section{INTRODUCTION}

Singlet oxygen $\left({ }^{1} \mathrm{O}_{2}\right)$ is a photochemically produced reactive intermediate (PPRI) and an important oxidant ubiquitous in sunlit aquatic environments. In natural and engineered systems, singlet oxygen is generated via energy transfer from triplet states of chromophoric dissolved organic matter (CDOM), the fraction of dissolved organic matter (DOM) able to absorb light that include aromatic ketones, coumarins, chromones, and quinones. ${ }^{1-5}$ In atmospheric aqueous phases such as fog, cloud, and rain droplets, ${ }^{1} \mathrm{O}_{2}$ can be produced via an analogous sensitization process involving atmospheric organic matter. ${ }^{6-11}$ In these diverse environments, ${ }^{1} \mathrm{O}_{2}$ contributes to the transformation of pollutants and biomolecules, ${ }^{1,12-16}$ to the deactivation of pathogens, ${ }^{17-21}$ to the formation and the photochemical aging of organic aerosols, $7,22,23$ and to the sunlight-induced oxidation of natural organic matter. ${ }^{24-26}$

In order to quantitatively assess the role of ${ }^{1} \mathrm{O}_{2}$ in these processes, one has to quantify the ability of natural chromophores to sensitize its formation. A useful parameter in this respect is the apparent singlet oxygen quantum yield $\left(\Phi_{\Delta}\right)$, which represents the moles of ${ }^{1} \mathrm{O}_{2}$ produced per moles of photons absorbed by a sensitizer (eq 1 ). In this context, the term "apparent" indicates that the actual sensitizer(s) responsible for ${ }^{1} \mathrm{O}_{2}$ production is unknown, a consequence of the complex molecular nature of CDOM. ${ }^{27-29}$

$$
\Phi_{\Delta}=\frac{\mathrm{mol}^{1} \mathrm{O}_{2} \text { formed }}{\text { mol photons absorbed }}
$$

The apparent ${ }^{1} \mathrm{O}_{2}$ quantum yield is an intensive property of CDOM, as it accounts for variations in sensitizers' absorption spectra and concentration, as well as for variations in light intensity. This feature makes $\Phi_{\Delta}$ a useful parameter in environmental chemistry studies, as it can be used to predict variations in ${ }^{1} \mathrm{O}_{2}$ steady-state concentrations as a function of light intensity (thus, water depth, presence of other water constituents, DOM concentration, seasonal light intensity fluctuations, etc.). ${ }^{2}$ Indeed, apparent quantum yields of PPRIs, including ${ }^{1} \mathrm{O}_{2}$, are needed as the input parameters in predictive models of steady-state concentrations and micropollutants' lifetimes. ${ }^{2,30,31}$ Furthermore, apparent quantum yields can also be used to study and compare the sensitizing properties of natural chromophores based on their origin (i.e., microbially vs terrestrially derived DOM $)^{8,32-39}$ and to understand the effect of environmental factors (e.g., photooxidation) on the photoreactivity of dissolved organic matter. ${ }^{40-43}$ Finally, the study of $\Phi_{\Delta}$ values offers the prospect of deepening our understanding of basic photophysical properties of DOM.

Despite the importance of apparent ${ }^{1} \mathrm{O}_{2}$ quantum yields, the array of techniques and large variability of experimental conditions employed over the years strongly limits a confident use of literature values for the above purposes. In this work, we reviewed 59 papers published between 1977 and March 2020 (Table 1) reporting apparent ${ }^{1} \mathrm{O}_{2}$ quantum yields of environmental samples, from natural and impacted aquatic environ-

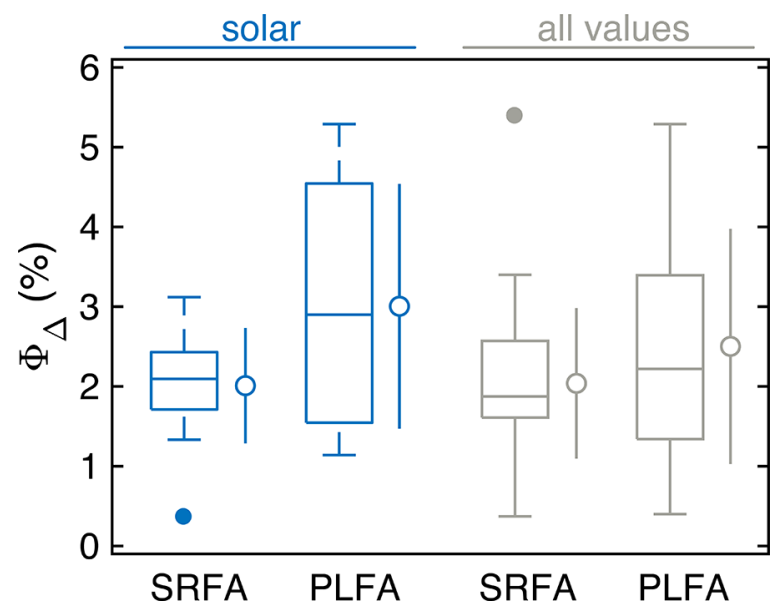

Figure 1. Comparison between apparent ${ }^{1} \mathrm{O}_{2}$ quantum yields of SRFA and PLFA across studies shown as boxplot and average (empty circles). Data in blue are solar-integrated quantum yields, while data in gray are all available values. In this analysis, we included only data for untreated DOM samples (database field "Treatment" = "none (isolate*)"). We did not consider potential variability in source material of different IHSS stocks (see section 3.2.2.5).

ments to atmospheric aquatic phases. We collected a total number of $857 \Phi_{\Delta}$ values ranging from $\approx 0 \%$ to $33 \%$ (median $=2.29 \%$ ). Even though several of these papers discuss trends and significant variations within their data sets, their conclusions often do not hold valid when $\Phi_{\Delta}$ values are compared across studies. Indeed, several independent studies measured higher $\Phi_{\Delta}$ for Pony Lake fulvic acid (PLFA), a microbial DOM end-member, compared to Suwannee River fulvic acid (SRFA), a terrestrial DOM end-member (Table 1). On average, $\Phi_{\Delta}^{\mathrm{PLFA}} / \Phi_{\Delta}^{\mathrm{SRFA}}=1.63 \pm 0.63$ when considering all studies $\left(N=13\right.$, median of $1.58 ; \Phi_{\Delta}^{\mathrm{PLFA}} / \Phi_{\Delta}^{\mathrm{SRFA}}=1.86 \pm 0.53$ when excluding the three values $<1$ ). However, if we pool all of the available data together, there appears to be no statistical difference between the $\Phi_{\Delta}$ values found for the two isolates. For instance, for data collected with the same light source (solar), the averages $\left( \pm\right.$ standard deviation) are $\Phi_{\Delta}=(2.0 \pm$ $0.7) \%(N=12)$ and $(3.0 \pm 1.5) \%(N=12)$ for SRFA and PLFA, respectively (Figure 1 ). Very comparable results are obtained from the average of all data, independent of the light source, i.e., $\Phi_{\Delta}=(2.0 \pm 0.9) \%(N=34)$ and $(2.5 \pm 1.5) \%(N$ $=18)$ for SRFA and PLFA, respectively. Similarly, several authors observed a strong positive correlation between E2:E3 (the ratio between the absorbance at 250 or $254 \mathrm{~nm}$ to that at $365 \mathrm{~nm})^{44,45}$ and $\Phi_{\Delta}$, but the slopes of the regression line vary considerably from one study to another, even if referred to the same light source (i.e., from 0.08 to 2.2 for xenon light; see section 5.2.2). From our point of view, these observations highlight the need of standardizing and better constraining the way we perform ${ }^{1} \mathrm{O}_{2}$ quantum yield measurements in environmental samples, in terms of general methodology, data treatment, data reporting, and quality control.

The first objective of this review is to critically evaluate the experimental methods that have been used to determine $\Phi_{\Delta}$ in environmental samples, with the specific aim of identifying potential sources of errors that might explain the large variations in literature values. Based on this analysis, we suggest guidelines and best practices to be considered for future studies. A second objective is to provide a comprehensive database of curated $\Phi_{\Delta}$ values of environ- 
Table 1. Overview of Apparent ${ }^{1} \mathrm{O}_{2}$ Quantum Yield Studies Found in the Environmental Photochemistry Literature from 1977 to March $2020^{a}$

\begin{tabular}{|c|c|c|c|}
\hline study & light source & $\Phi_{\Delta}^{\mathrm{SRFA}}(\%)$ & $\Phi_{\Delta}^{\mathrm{PLFA}}(\%)$ \\
\hline \multicolumn{4}{|c|}{ chemical probe, absolute, radiometry } \\
\hline \multicolumn{4}{|l|}{ UVC } \\
\hline Lester et al., 2013 & mercury $(255 \mathrm{~nm})$ & $3.20 \pm 0.05$ & \\
\hline \multicolumn{4}{|l|}{ UVB and UVA } \\
\hline Haag et al., 1984 & xenon $(365,405,545 \mathrm{~nm})$ & & \\
\hline \multirow[t]{2}{*}{ Frimmel et al., 1987} & xenon $(365 \mathrm{~nm})$ & $1.00 \pm 0.30(\mathrm{pH} \mathrm{7})$ & \\
\hline & & $1.80 \pm \underset{3.5)}{0.54}(\mathrm{pH}$ & \\
\hline Dalrymple et al., $2010^{b}$ & mercury $(365 \mathrm{~nm})$ & & \\
\hline Nkhili et al., $2014^{c}$ & UVA bulbs $(365 \mathrm{~nm})$ & & \\
\hline Sharpless et al., $2014^{b}$ & mercury $(365 \mathrm{~nm})$ & 1.8 & \\
\hline Hong et al., 2018 & mercury $(290-450 \mathrm{~nm})$ & $1.71 \pm 0.02$ & \\
\hline Li et al., 2019 & UVA bulbs $(380 \mathrm{~nm})$ & & \\
\hline Zhao et al., 2020 & mercury $(290-450 \mathrm{~nm})$ & $1.61 \pm 0.12$ & \\
\hline Zhou et al., 2020 & mercury $(290-450 \mathrm{~nm})$ & $1.23 \pm 0.05$ & $2.90 \pm 0.11$ \\
\hline \multicolumn{4}{|l|}{$\begin{array}{l}\text { solar } \\
\text { Glover and Rosario-Ortiz } 2013\end{array}$} \\
\hline Glover and Rosario-Ortiz, 2013 & xenon (solar) & 0.37 & 1.14 \\
\hline Mostafa and Rosario-Ortiz, 2013 & xenon (solar) & $2.11 \pm 0.31^{d}$ & $2.04 \pm 0.27^{d}$ \\
\hline Cawley et al., 2014 & xenon (solar) & & \\
\hline Mostafa et al., 2014 & xenon (solar) & & \\
\hline Zhang et al., 2014 & xenon (solar) & $1.85 \pm 0.15^{d}$ & $1.34 \pm 0.09^{d}$ \\
\hline Fu et al., 2016 & xenon (solar) & & \\
\hline McKay et al., 2016 & xenon (solar) & $1.8 \pm 0.1$ & \\
\hline McKay et al., 2017 & xenon (solar) & & \\
\hline Silva et al., 2017 & xenon (solar), mercury (255 nm) & & \\
\hline Tenorio et al., 2017 & xenon (solar), cutoff at $310 \mathrm{~nm}$ & & \\
\hline Zhou et al., 2017 & xenon (solar) & $3.12 \pm 0.22$ & $4.55 \pm 0.26$ \\
\hline Zhou et al., 2018 & xenon (solar) & $2.83 \pm 0.07$ & $4.96 \pm 0.11$ \\
\hline Chen et al., 2019 & xenon (solar) & & \\
\hline Wang et al., 2019 & xenon (solar) & $2.2 \pm 0.1$ & \\
\hline Wenk et al., 2019 & xenon (solar), cutoff at $320 \mathrm{~nm}$ & $1.61 \pm 0.12^{d}$ & $1.55 \pm 0.12^{d}$ \\
\hline
\end{tabular}

chemical probe, absolute, actinometry

UVB and UVA

Zepp et al., 1977

Baxter and Carey, 1982

Zepp et al., 1985

Faust and Allen, 1992

Aguer et al., 1997

Halladja et al., 2007

Sharpless, 2012

Janssen et al., 2014

Bodhipaksha et al., 2015

Marchisio et al., 2015

Bodhipaksha et al., 2017

Maizel and Remucal, 2017 (ES\&T)

Maizel and Remucal, 2017 (ESPI)

Maizel et al., 2017

Berg et al., 2019

mercury $(365 \mathrm{~nm})$

mercury $(365 \mathrm{~nm})$

mercury $(315,365,405 \mathrm{~nm})$

xenon $(335 \mathrm{~nm})$

mercury $(365 \mathrm{~nm})$

xenon $(320,340,375,400,425,450,500 \mathrm{~nm})$

xenon $(310,370,415 \mathrm{~nm})$

UVB bulbs $(315 \mathrm{~nm})$

mercury $(365 \mathrm{~nm})$

UVB bulbs $(315 \mathrm{~nm})$, UVA bulbs $(365 \mathrm{~nm})$, blue bulbs $(420 \mathrm{~nm})$

Mercury $(290-450 \mathrm{~nm})$

UVA bulbs $(365 \mathrm{~nm})$

UVA bulbs $(365 \mathrm{~nm})$

UVA bulbs $(365 \mathrm{~nm})$

UVA bulbs $(365 \mathrm{~nm})$

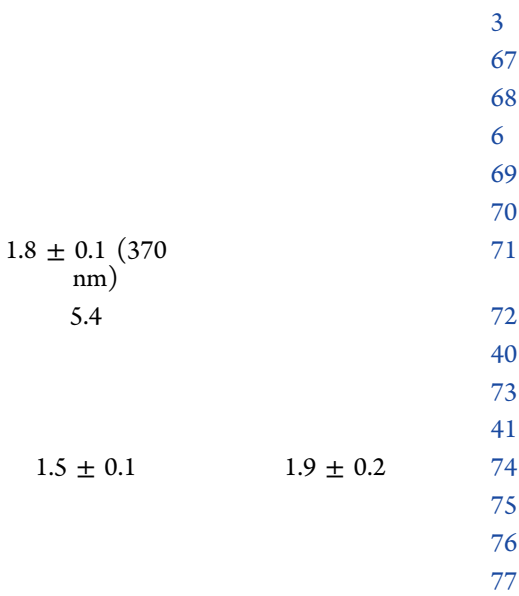

solar

xenon (solar), cutoff at $310 \mathrm{~nm}$

\begin{tabular}{|c|c|}
\hline 2.14 & 3.4 \\
\hline $1.33 \pm 0.07(\mathrm{pH} 7)$ & $2.53 \pm 0.14(\mathrm{pH} 7)$ \\
\hline $2.08 \pm 0.11(\mathrm{pH} 3)$ & $3.27 \pm 0.18(\mathrm{pH} \mathrm{3})$ \\
\hline
\end{tabular}

xenon (solar), cutoff at $290 \mathrm{~nm}$ $2.08 \pm 0.11(\mathrm{pH} 3) \quad 3.27 \pm 0.18(\mathrm{pH} 3)$

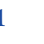

.

65

Laurentiis et al., 2013

McCabe and Arnold, 2016

xenon (solar), cutoff at $295 \mathrm{~nm}$

xenon (solar)

xenon (solar), cutoff at $295 \mathrm{~nm}$

xenon (solar),

O'Connor et al., 2019

Wan et al., 2019

xenon (solar), cutoff at $300 \mathrm{~nm}$ 
Table 1. continued

\begin{tabular}{|c|c|c|c|c|}
\hline study & light source & $\Phi_{\Delta}^{\mathrm{SRFA}}(\%)$ & $\Phi_{\Delta}^{\mathrm{PLFA}}(\%)$ & ref \\
\hline Zhou et al., 2019 & xenon (solar), cutoff at $290 \mathrm{~nm}$ & $2.66 \pm 0.12$ & $5.29 \pm 0.12$ & 80 \\
\hline \multicolumn{5}{|l|}{ chemical probe, relative } \\
\hline Cory et al., 2009 & UVA bulbs $(365 \mathrm{~nm})$ & 0.47 & 0.69 & 81 \\
\hline Peterson et al., 2012 & UVA bulbs $(365 \mathrm{~nm})$ & & & 36 \\
\hline Chu et al., 2015 & UVA bulbs $(365 \mathrm{~nm})$ & & & 82 \\
\hline \multirow[t]{2}{*}{ Manfrin et al., 2019} & UVA bulbs $(365 \mathrm{~nm})$, UVB bulbs $(310 \mathrm{~nm})$ & $1.9 \pm 0.6(\mathrm{UVA})$ & & 9 \\
\hline & & $3.4 \pm 1.1(\mathrm{UVB})$ & & \\
\hline \multicolumn{5}{|c|}{${ }^{1} \mathrm{O}_{2}$ phosphorescence, time-resolved } \\
\hline Paul et al, 2004 & Nd:YAG laser $(350,450,480,500,550 \mathrm{~nm})$ & $0.57(480 \mathrm{~nm})$ & & 83 \\
\hline Carlos et al., 2012 & Ti:sapphire laser $(400 \mathrm{~nm})$ & & & 84 \\
\hline Pozdnyakov et al., 2017 & Nd:YAG laser $(532 \mathrm{~nm})$ & & & 85 \\
\hline Partanen et al., 2020 & $\begin{array}{l}\text { Ti:sapphire laser }(300,320,330,340,365,380,400,430,470,500 \text {, } \\
\quad 540,560 \mathrm{~nm})\end{array}$ & $1.4(365 \mathrm{~nm})$ & $2.4(365 \mathrm{~nm})$ & 39 \\
\hline \multicolumn{5}{|c|}{${ }^{1} \mathrm{O}_{2}$ phosphorescence, steady-state } \\
\hline Sandvik et al., 2000 & mercury $(280-380 \mathrm{~nm})$ & & & 37 \\
\hline Bilski et al., 2003 & mercury $(365 \mathrm{~nm})$ & & & 86 \\
\hline
\end{tabular}

${ }^{a}$ Studies are divided into five groups according to the classification provided in Figure 3 and are ordered chronologically within each group. For absolute chemical probe studies, we further subdivided the papers based on the light source. When available, we report $\Phi_{\Delta}$ values for SRFA and PLFA to provide a better sense of the variability observed also under comparable experimental conditions. ${ }^{b}$ Some measurements were performed via a reference chemical probe method (Table S8). ${ }^{c}$ Unclear if authors used radiometry or chemical actinometry. ${ }^{d}$ Exceptions of the general trend $\Phi_{\Delta}^{\mathrm{SRFA}}<\Phi_{\Delta}^{\mathrm{PLFA}}$

mentally relevant samples. Even within the limitations of the current methods, we believe that this data set still represents a valuable resource for modelers and other scientists interested in understanding and characterizing the natural variability of DOM photoreactivity. Finally, we provide an overview of results accumulated over more than 40 years of $\Phi_{\Delta}$ research, including $\Phi_{\Delta}$ trends as a function of relevant water chemistry parameters, spectroscopic and molecular DOM features, light sources, and irradiation wavelengths. In this final section, we also revise available data on spatial and temporal variability in apparent ${ }^{1} \mathrm{O}_{2}$ quantum yields.

\section{BRIEF OVERVIEW OF SURFACE WATER ${ }^{1} \mathrm{O}_{2}$ PHOTOPHYSICS}

Here we provide a brief summary of basic photophysical processes pertaining to ${ }^{1} \mathrm{O}_{2}$ in natural systems (Figure 2). A more detailed discussion of its photophysical properties and of sensitized ${ }^{1} \mathrm{O}_{2}$ production in biological, environmental, and photocatalytic systems can be found elsewhere. ${ }^{1,4,87-93}$ In this review, we use the term CDOM to indicate any complex mixture of organic molecules present in the aquatic environment (i.e., surface waters but also the atmospheric aqueous phases) that are able to absorb sunlight. Note that the same scheme is also valid for discrete-molecule sensitizers (Sens) such as the ones used as $\Phi_{\Delta}$ reference compounds (e.g., perinaphthenone).

Upon absorption of light $(h \nu), \mathrm{CDOM}$ is promoted to its singlet excited state, ${ }^{1} \mathrm{CDOM}^{*}$. ${ }^{1} \mathrm{CDOM}^{*}$ is a short-lived intermediate (estimated lifetime of 150 ps to $6 \mathrm{~ns}$ ), ${ }^{94}$ which either relaxes back to its ground state via internal conversion (IC; nonradiative deactivation) or fluorescence emission (F; radiative deactivation), or undergoes intersystem crossing (ISC) that converts it to a triplet excited state $\left({ }^{3} \mathrm{CDOM}^{*}\right)$. The (apparent) intersystem crossing quantum yield or ${ }^{3} \mathrm{CDOM}^{*}$ quantum yield $\left(\Phi_{\mathrm{T}}\right)$ describes the conversion efficiency of ${ }^{1} \mathrm{CDOM}^{*}$ into ${ }^{3} \mathrm{CDOM}^{*}$. Compared to the singlet excited state, ${ }^{3} \mathrm{CDOM}^{*}$ has a longer lifetime (10-30 $\mu \mathrm{s})^{95}$ and can participate in bimolecular chemical reactions before decaying back to the ground state. Depending on the substrate, ${ }^{3} \mathrm{CDOM}^{*}$ can act as an oxidant (electron transfer reactions) or undergo energy transfer reactions; ${ }^{5}$ alternatively, ${ }^{3} \mathrm{CDOM}^{*}$ can relax back to the ground state via radiative (phosphorescence emission; P) or nonradiative processes (i.e., intersystem crossing) with a first-order rate constant $k_{\mathrm{d}}^{\mathrm{T}}$, which is equivalent to the inverse of the natural triplet lifetime (the term "natural" indicates the lifetime in the absence of quenchers). Due to its relatively high concentrations in sunlit aquatic systems compared to other natural ${ }^{3} \mathrm{CDOM}^{*}$ quenchers $\left(\sim 260 \mu \mathrm{mol} \mathrm{L}{ }^{-1} \text { at } 25{ }^{\circ} \mathrm{C}\right)^{96}$ and its high reactivity with excited triplet states, oxygen is the most prominent ${ }^{3} \mathrm{CDOM}^{*}$ quencher, and is thus the species that largely controls its lifetime in the natural environment. ${ }^{5}$ Upon energy transfer, $\mathrm{O}_{2}$ is converted to singlet oxygen $\left({ }^{1} \mathrm{O}_{2}\right)$, while ${ }^{3} \mathrm{CDOM}^{*}$ decays back to its (singlet) ground state. In principle, energy transfer from triplet sensitizers can produce ${ }^{1} \mathrm{O}_{2}$ in two excited states, i.e., $\mathrm{O}_{2}\left({ }^{1} \Delta_{\mathrm{g}}\right)$ and $\mathrm{O}_{2}\left({ }^{1} \Sigma_{\mathrm{g}}^{+}\right)$, which are 94 and $157 \mathrm{~kJ} \mathrm{~mol}^{-1}$, respectively, above $\mathrm{O}_{2}$ (not indicated in Figure 2; see Paterson et al. ${ }^{97}$ for more information on the symbols ${ }^{1} \Delta_{\mathrm{g}}$ and $\left.{ }^{1} \Sigma_{\mathrm{g}}^{+}\right) .{ }^{89,98}$ However, due to the extremely efficient deactivation of $\mathrm{O}_{2}\left({ }^{1} \Sigma_{\mathrm{g}}^{+}\right)$to $\mathrm{O}_{2}\left({ }^{1} \Delta_{\mathrm{g}}\right)$, the relevance of the ${ }^{1} \Sigma_{\mathrm{g}}^{+}$state is negligible in most systems. ${ }^{89}$ Thus, in this review, ${ }^{1} \mathrm{O}_{2}$ indicates only the first singlet oxygen excited state, i.e., $\mathrm{O}_{2}\left({ }^{1} \Delta_{\mathrm{g}}\right)$. Furthermore, ${ }^{1} \mathrm{O}_{2}$ can also be produced from the sensitizer's singlet excited state (i.e., $\left.{ }^{1} \mathrm{CDOM}^{*}\right)^{89,98,99}$ and from direct excitation of $\mathrm{O}_{2}$ at $765 \mathrm{~nm}^{100,101}$ (not indicated in Figure 2). To the authors' knowledge, ${ }^{1} \mathrm{O}_{2}$ production via these two additional mechanisms remains untested in the context of aquatic photochemistry. The first-order rate constant of the triplet-sensitized production of ${ }^{1} \mathrm{O}_{2}$ can be obtained as $k_{\mathrm{O} 2}\left[\mathrm{O}_{2}\right] f_{\Delta}$, where $k_{\mathrm{O} 2}$ is the second-order rate constants for the physical quenching of ${ }^{3} \mathrm{CDOM}^{*}$ with $\mathrm{O}_{2},\left[\mathrm{O}_{2}\right]$ is the dissolved oxygen concentration, and $f_{\Delta}$ is the fraction of physical quenching that leads to ${ }^{1} \mathrm{O}_{2}$ production. Note that a fraction $1-f_{\Delta}$ of all oxygen quenching events deactivates 


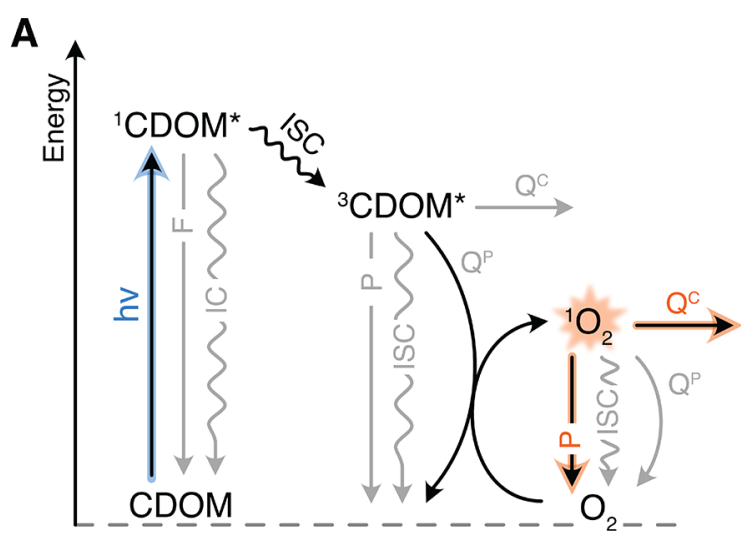

B

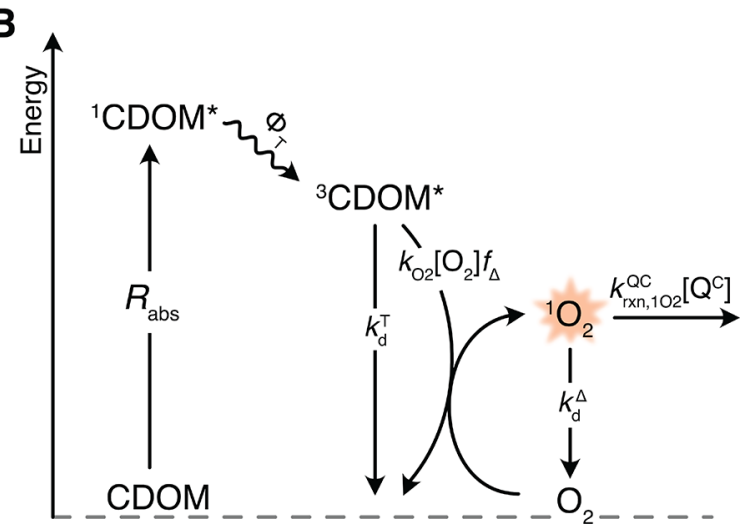

Figure 2. Schematic overview of the photophysical properties of CDOM and ${ }^{1} \mathrm{O}_{2}$. Panel A shows a qualitative overview of all possible photophysical processes, while quantitative parameters relevant for understanding ${ }^{1} \mathrm{O}_{2}$ photophysics are indicated in panel $\mathbf{B}$. In panel $\mathbf{A}$, gray arrows indicate processes that occur but are not directly relevant for ${ }^{1} \mathrm{O}_{2}$ measurements; colored arrows indicate processes that are directly monitored in $\Phi_{\Delta}$ measurements; straight arrows are radiative decays or chemical reactions, while wavy arrows are nonradiative processes. Legend for panel A: F, fluorescence; IC, internal conversion; ISC, intersystem crossing; $\mathrm{P}$, phosphorescence; $\mathrm{Q}^{\mathrm{C}}$, chemical quencher; $\mathrm{Q}^{\mathrm{P}}$, physical quencher. Legend for panel $\mathbf{B}: R_{\mathrm{abs}}$, rate of light absorption; $\Phi_{\mathrm{T}}$, apparent ${ }^{3} \mathrm{CDOM}^{*}$ quantum yield; $k_{\mathrm{d}}^{\mathrm{T}}$, (natural) ${ }^{3} \mathrm{CDOM}^{*}$ decay rate constant; $k_{\mathrm{d}}^{\Delta}$, (natural) ${ }^{1} \mathrm{O}_{2}$ decay rate constant; $k_{\mathrm{O} 2}$, second-order rate constants for the physical quenching of ${ }^{3} \mathrm{CDOM}^{*}$ with $\mathrm{O}_{2} ; f_{\Delta}$, fraction of physical quenching that leads to ${ }^{1} \mathrm{O}_{2}$ production; $k_{\mathrm{rxn}, 1 \mathrm{O} 2}^{\mathrm{QC}}$, second-order rate constant for the reaction of $\mathrm{Q}^{\mathrm{C}}$ with ${ }^{1} \mathrm{O}_{2}$.

${ }^{3} \mathrm{CDOM}^{*}$ without resulting in the formation of singlet oxygen (not indicated in Figure 2B). Similar to ${ }^{3} \mathrm{CDOM}^{*}$, singlet oxygen is a transient species with a natural lifetime on the order of microseconds in distilled water $(\sim 3.5 \mu \mathrm{s}) .{ }^{101-103} \mathrm{In}$ the presence of a chemical quencher $\left(\mathrm{Q}^{\mathrm{C}}\right),{ }^{1} \mathrm{O}_{2}$ can chemically react to form endoperoxides and ring-opened products with a second-order rate constant $k_{\mathrm{rxn}, 1 \mathrm{O} 2}^{\mathrm{QC}} \cdot{ }^{1,104}$ Alternatively, ${ }^{1} \mathrm{O}_{2}$ can undergo energy-transfer quenching (i.e., physical quenchers, $\mathrm{Q}^{\mathrm{P}}$, such as with tertiary aliphatic amines) ${ }^{105}$ and be converted back to its ground state. In the absence of quenchers, ${ }^{1} \mathrm{O}_{2}$ can decay back to the ground state via radiative (phosphorescence emission at $1270 \mathrm{~nm}$ ) and nonradiative pathways (intersystem crossing) with a first-order rate constant $k_{\mathrm{d}}^{\Delta}$, which represents the inverse of the natural ${ }^{1} \mathrm{O}_{2}$ lifetime. Notably, $k_{\mathrm{d}}^{\Delta}$ changes considerably from $\mathrm{D}_{2} \mathrm{O}\left(1 / k_{\mathrm{d}}^{\Delta}=\tau_{\Delta} \approx 67 \mu \mathrm{s}\right)$ to $\mathrm{H}_{2} \mathrm{O}\left(\tau_{\Delta} \approx 3.5\right.$ $\mu \mathrm{s})$ due to vibrational energy differences between $\mathrm{H}-\mathrm{O}$ and $\mathrm{D}-\mathrm{O}$ bonds. ${ }^{89,102,106,107}$ This fact has been exploited in several contexts either as a diagnostic test for ${ }^{1} \mathrm{O}_{2}$ involvement in chemical reactions ${ }^{29,90,102}$ or as a way to overcome the low time-resolution and sensitivity of the first singlet oxygen phosphorescence detectors (vide infra).

From a quantitative point of view, the singlet oxygen quantum yield of a sensitizer can be expressed as a function of photophysical and chemical properties of the system according to eq $2^{71,89,98}$

$$
\Phi_{\Delta}=\Phi_{\mathrm{T}}\left(\frac{k_{\mathrm{O} 2}\left[\mathrm{O}_{2}\right]}{k_{\mathrm{d}}^{\mathrm{T}}+k_{\mathrm{O} 2}\left[\mathrm{O}_{2}\right]}\right) f_{\Delta}
$$

This equation shows that $\Phi_{\Delta}$, which is the fraction of excited CDOM that produces ${ }^{1} \mathrm{O}_{2}$, is itself a product of three fractions: the fraction of ${ }^{1} \mathrm{CDOM}^{*}$ that becomes ${ }^{3} \mathrm{CDOM}^{*}\left(\Phi_{\mathrm{T}}\right)$, the fraction of triplets that are quenched by oxygen $\left(k_{\mathrm{O} 2}\left[\mathrm{O}_{2}\right] /\right.$ $\left.\left(k_{\mathrm{d}}^{\mathrm{T}}+k_{\mathrm{O} 2}\left[\mathrm{O}_{2}\right]\right)\right)$, and the fraction of these quenching events that lead to formation of ${ }^{1} \mathrm{O}_{2}\left(f_{\Delta}\right)$. In theory, eq 2 should contain an additional term that accounts for the production of ${ }^{1} \mathrm{O}_{2}$ from the quenching of ${ }^{1} \mathrm{CDOM}^{*} .{ }^{87,89,99}$ Considering the sizable difference in triplet and singlet excited state lifetimes (i.e., 150 ps to $6 \mathrm{~ns}^{94}$ vs 10 to $30 \mu \mathrm{s}^{95}$ for ${ }^{1} \mathrm{CDOM}^{*}$ and ${ }^{3} \mathrm{CDOM}^{*}$, respectively) and the relatively low $\mathrm{O}_{2}$ concentrations in surface waters compared to organic solvents, this contribution is expected to be minor. We also point out that $\Phi_{\Delta}<\Phi_{\mathrm{T}}$, as $k_{\mathrm{d}}^{\mathrm{T}}>0$, and that apparent ${ }^{1} \mathrm{O}_{2}$ quantum yields depend on dissolved oxygen concentrations (see also section 5.1).

Based on the kinetic scheme in Figure 2A, it is possible to recognize the relevant photophysical processes that need to be monitored to measure apparent ${ }^{1} \mathrm{O}_{2}$ quantum yields. The first important parameter is the number of photons absorbed by the chromophore (the denominator of eq 1 , blue shaded arrow in Figure $2 \mathrm{~A}$ ). In $\Phi_{\Delta}$ determinations, this parameter is generally quantified as the rate of light absorption $\left(R_{\mathrm{abs}}\right.$, in (mol absorbed photons) $L^{-1} s^{-1}$ ), which represents the number of absorbed photons per unit of time and volume. $R_{\mathrm{abs}}$ is proportional to the overlap between the absorption spectrum of the sensitizer and the emission spectrum of the light source (more details in section 3.2.1.1). A second important quantity is the moles of ${ }^{1} \mathrm{O}_{2}$ produced (the numerator of eq 1 ), which can be estimated in several ways (orange shaded arrows in Figure 2A). A common technique involves the use of a probe compound (a chemical quencher) that can trap ${ }^{1} \mathrm{O}_{2}$. From the probe depletion kinetics, it is possible to calculate the amount of photogenerated ${ }^{1} \mathrm{O}_{2}$. Alternatively, one can observe ${ }^{1} \mathrm{O}_{2}$ directly by monitoring its phosphorescence emission $1270 \mathrm{~nm}$. These two approaches are the basis of the techniques that have been used to measure $\Phi_{\Delta}$ in environmental samples and are described more in detail in the following section.

\section{METHODS FOR $\Phi_{\Delta}$ DETERMINATION IN ENVIRONMENTAL SYSTEMS}

There are two well-defined experimental strategies that have been used to measure $\Phi_{\Delta}$ in environmental samples. As introduced in the discussion above, the first approach consists of quantifying ${ }^{1} \mathrm{O}_{2}$ via reaction with a chemical probe, while the second method is based on direct observation of ${ }^{1} \mathrm{O}_{2}$ via its weak phosphorescence signal at $1270 \mathrm{~nm}$ (Figure 3). Within each category, we identified further distinctions based on minor variations in the experimental approach. Chemical probe methods are classified as absolute or relative based on the strategy employed to quantify the rate of light absorption 

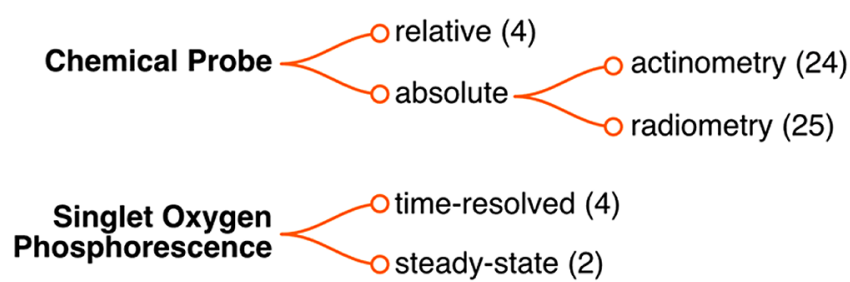

Figure 3. Schematic of the main methods to quantify ${ }^{1} \mathrm{O}_{2}$ quantum yields in environmental samples classified as chemical probe and singlet oxygen phosphorescence methods. In parentheses we report the number of literature studies per each subgroup (see also Table 1). Note that different chemical probe methods are classified based on the strategy used to measure the absolute spectral irradiance, which is needed to calculate the rate of light absorption.

$\left(R_{\mathrm{abs}}\right)$. Absolute probe-based methods require the explicit calculation of $R_{\mathrm{abs}}$; in contrast, relative methods avoid this step by using a reference ${ }^{1} \mathrm{O}_{2}$ sensitizer whose $\Phi_{\Delta}$ is well-known. Absolute probe-based methods can be further divided into two groups based on how the absolute spectral irradiance is determined, either by chemical actinometry or radiometry. On the other hand, phosphorescence-based methods can be classified as steady-state or time-resolved based on the experimental setup used to produce and detect the ${ }^{1} \mathrm{O}_{2}$ phosphorescence signal. To date, there have been no timeresolved probe-based methods, as all of the reported experiments have determined apparent ${ }^{1} \mathrm{O}_{2}$ quantum yields via steady-state irradiation. Likewise, there have been no absolute phosphorescence-based measurements, as all those reported so far have been relative to a reference sensitizer.

Since the first report in $1977,{ }^{3}$ probe-based methods have been the technique of choice for measurements of apparent ${ }^{1} \mathrm{O}_{2}$ quantum yields of environmental samples due to the straightforward experimental setup and protocol (53 of 59 studies; 90\%), with actinometry (24 of 59; $41 \%$ ) and radiometry ( 25 of 59 ; $42 \%$ ) being equally popular variants. Despite being more robust (see discussion in section 3.3), relative chemical probe methods have only rarely been used ( 4 studies over 59; 7\%). A clear disadvantage of all probe-based methods is that they detect ${ }^{1} \mathrm{O}_{2}$ indirectly and are thus susceptible to interference from other photochemically produced reactive intermediates (see section 3.2.2.4). Similarly to the relative chemical probe method, ${ }^{1} \mathrm{O}_{2}$ phosphorescence has not yet gained wide popularity among the environmental chemistry community ( 6 of $59 ; 10 \%$ ), probably because of the more specialized and expensive equipment demands, both in terms of light sources (e.g., Nd:YAG pulsed lasers for the timeresolved methods) and high-sensitivity detectors (e.g., $77 \mathrm{~K}$ cooled Ge diode), and the requisite use of highly concentrated DOM solutions. Note also that, until the most recent generation of thermoelectrically cooled NIR photomultiplier tubes, these experiments had to be conducted in $\mathrm{D}_{2} \mathrm{O}$ to overcome the low sensitivity and poor time resolution of $\mathrm{Ge}$ photodiode detectors, ${ }^{89,108,109}$ which might have further lowered the general appeal of this approach (more details in section 3.1.2). This disadvantage is a direct consequence of the low ${ }^{1} \mathrm{O}_{2}$ phosphorescence emission quantum yield in $\mathrm{H}_{2} \mathrm{O}$ (i.e., $\left.<10^{-6}\right) .{ }^{89}$ On the other hand, ${ }^{1} \mathrm{O}_{2}$ phosphorescence is a more selective technique than probe-based methods because it detects the near-infrared emission that is unique to this reactive species, thus it is not prone to interferences from other PPRIs. ${ }^{90}$
In the following sections, we derive the general $\Phi_{\Delta}$ equations needed for each of the methods in Figure 3 (section 3.1 ), we critically discuss all of the important parameters that are part of the equations (section 3.2), we qualitatively summarize and estimate the magnitude of most common errors in ${ }^{1} \mathrm{O}_{2}$ quantum yield determinations (section 3.3), and we provide general guidelines for future studies (section 3.4). Due to the ease of application and widespread use in the literature, our discussion will be more oriented toward probebased methods.

\subsection{Derivation of the Basic Equations}

3.1.1. Chemical Probe Methods. Chemical probe methods involve the use of a probe compound $(\mathrm{P})$ that reacts selectively with ${ }^{1} \mathrm{O}_{2}$ (eq 3). Furfuryl alcohol (FFA) is the most widely used ${ }^{1} \mathrm{O}_{2}$ probe in environmental studies, ${ }^{1,103}$ even if alternative compounds have been developed and used in the past $^{1,110,111}$ and in other fields. ${ }^{29,112-117}$

$$
\mathrm{P}+{ }^{1} \mathrm{O}_{2} \stackrel{k_{\mathrm{rxn}, 102}^{\mathrm{P}}}{\longrightarrow} \text { products }
$$

The rate of disappearance of $\mathrm{P}$ can be written according to eq 4.

$$
\begin{aligned}
-\frac{\mathrm{d}[\mathrm{P}]}{\mathrm{d} t} & =k_{\mathrm{rxn}, 1 \mathrm{O} 2}^{\mathrm{P}}\left[{ }^{1} \mathrm{O}_{2}\right]_{\mathrm{ss}}[\mathrm{P}] \\
& =R_{\mathrm{abs}} \Phi_{\Delta} \frac{k_{\mathrm{rxn}, 1 \mathrm{O} 2}^{\mathrm{P}}[\mathrm{P}]}{k_{\mathrm{d}}^{\Delta}+\left(k_{\mathrm{phys}, 1 \mathrm{O} 2}^{\mathrm{P}}+k_{\mathrm{rxn}, 1 \mathrm{O} 2}^{\mathrm{P}}\right)[\mathrm{P}]}
\end{aligned}
$$

where $R_{\mathrm{abs}}$ ((mol absorbed photons) $\left.\mathrm{L}^{-1} \mathrm{~s}^{-1}\right)$ is the rate of light absorption of the sensitizer, either CDOM or a singlemolecule sensitizer, $\Phi_{\Delta}$ is the apparent ${ }^{1} \mathrm{O}_{2}$ quantum yield, $k_{\mathrm{rxn}, 1 \mathrm{O} 2}^{\mathrm{P}}\left(\mathrm{L} \mathrm{mol}^{-1} \mathrm{~s}^{-1}\right)$ is the bimolecular rate constant for the reaction of the probe molecule with ${ }^{1} \mathrm{O}_{2}, k_{\text {phys,102 }}^{\mathrm{P}}\left(\mathrm{L} \mathrm{mol}^{-1} \mathrm{~s}^{-1}\right)$ is the bimolecular rate constant for physical quenching of the $\mathrm{P}$ with ${ }^{1} \mathrm{O}_{2}$, and $k_{\mathrm{d}}^{\Delta}\left(\mathrm{s}^{-1}\right)$ is the singlet oxygen deactivation rate constant to ground state $\mathrm{O}_{2} \cdot{ }^{48,118}$ In this equation, degradation via direct photolysis and reaction with other PPRIs is assumed to be negligible. In addition, this equation assumes that the only deactivation processes for ${ }^{1} \mathrm{O}_{2}$ are solvent-dependent deactivation and quenching by the probe compound, which is usually the case.

If $\left(k_{\text {phys }, 1 \mathrm{O} 2}^{\mathrm{P}}+k_{\mathrm{rxn}, 1 \mathrm{O} 2}^{\mathrm{P}}\right)[\mathrm{P}] \ll k_{\mathrm{d}}^{\Delta}$, probe molecule loss follows pseudo-first order kinetics (eq 5), where $k_{\mathrm{obs}, \mathrm{P}}\left(\mathrm{s}^{-1}\right)$ is defined according to eq 6 .

$$
\begin{aligned}
& -\frac{\mathrm{d}[\mathrm{P}]}{\mathrm{d} t}=\frac{R_{\mathrm{abs}} \Phi_{\Delta} k_{\mathrm{rxn}, 1 \mathrm{O} 2}^{\mathrm{P}}}{k_{\mathrm{d}}^{\Delta}}[\mathrm{P}]=k_{\mathrm{obs}, \mathrm{P}}[\mathrm{P}] \\
& k_{\mathrm{obs}, \mathrm{P}}=k_{\mathrm{rxn}, 1 \mathrm{O} 2}^{\mathrm{P}}\left[{ }^{1} \mathrm{O}_{2}\right]_{\mathrm{ss}}=\frac{R_{\mathrm{abs}} \Phi_{\Delta} k_{\mathrm{rxn}, 1 \mathrm{O} 2}^{\mathrm{P}}}{k_{\mathrm{d}}^{\Delta}}
\end{aligned}
$$

Equation 6 can be rearranged to provide an expression for the singlet oxygen quantum yield (eq 7).

$$
\Phi_{\Delta}=\frac{k_{\mathrm{obs}, \mathrm{P}}}{k_{\mathrm{rxn}, 1 \mathrm{O} 2}^{\mathrm{P}}} \frac{k_{\mathrm{d}}^{\Delta}}{R_{\mathrm{abs}}}
$$

Equation 7 represents the starting point of three probe-based methods and is also the basic equation for absolute quantum yields determinations. Note that this equation is valid under the assumption that $\left(k_{\mathrm{phys}, 1 \mathrm{O} 2}^{\mathrm{P}}+k_{\mathrm{rxn}, 1 \mathrm{O} 2}^{\mathrm{P}}\right)[\mathrm{P}] \ll k_{\mathrm{d}}^{\Delta}$, thus that $[\mathrm{P}]$ is low enough to not influence the ${ }^{1} \mathrm{O}_{2}$ steady-state concentration. So far, most apparent ${ }^{1} \mathrm{O}_{2}$ quantum yields 
determinations in environmental samples have been based on this approximation, even if this not the only possible approach for data analysis. For example, in early $\Phi_{\Delta}$ measurements, P was deliberately added at high concentrations to trap all the photogenerated ${ }^{1} \mathrm{O}_{2} \cdot{ }^{33,47}$ Alternatively, a few authors adopted the initial rate approach based on zeroth-order kinetics. $^{38,49,52,69,70,73}$ Details on data analysis approaches can be found in section 3.2.2.2. Note that this review focuses on $\Phi_{\Delta}$ measurements in environmental samples; readers interested in steady-state ${ }^{1} \mathrm{O}_{2}$ quantum yield determinations in other systems should refer to other reviews on the subject. ${ }^{29,98}$

Even within the steady-state approach, several variants can be found in the literature. Indeed, authors have expressed eq 7 in terms of ${ }^{1} \mathrm{O}_{2}$ formation rates $\left(R_{1 \mathrm{O}_{2}}\right.$; eq $8){ }^{7,8,32,34,35,40,41,43,46,48,51,55,57,59,62-64,80}$ steady-state concentrations $\left(\left[{ }^{1} \mathrm{O}_{2}\right]_{\mathrm{ss}}\right.$, eq 9), ${ }^{42,54,56,61,66,72,78}$ quantum yield coefficients $\left(f_{\mathrm{P}}\right.$; eq 10$),{ }^{75}$ or beta factors $(\beta$, eq 11$) .{ }^{3,68}$

$$
\begin{aligned}
& \Phi_{\Delta}=\frac{R_{1 \mathrm{O} 2}}{R_{\mathrm{abs}}} ; R_{1 \mathrm{O} 2}=\frac{k_{\mathrm{d}}^{\Delta} k_{\mathrm{obs}, \mathrm{P}}}{k_{\mathrm{rxn}, 1 \mathrm{O} 2}^{\mathrm{P}}} \\
& \Phi_{\Delta}=\left[{ }^{1} \mathrm{O}_{2}\right]_{\mathrm{ss}} \frac{k_{\mathrm{d}}^{\Delta}}{R_{\mathrm{abs}}} ;\left[{ }^{1} \mathrm{O}_{2}\right]_{\mathrm{ss}}=\frac{k_{\mathrm{obs}, \mathrm{P}}}{k_{\mathrm{rxn}, 1 \mathrm{O} 2}^{\mathrm{P}}} \\
& \Phi_{\Delta}=f_{\mathrm{P}} \frac{k_{\mathrm{d}}^{\Delta}}{k_{\mathrm{rxn}, 1 \mathrm{O} 2}^{\mathrm{P}}} ; f_{\mathrm{P}}=\frac{k_{\mathrm{obs}, \mathrm{P}}}{R_{\mathrm{abs}}} \\
& \Phi_{\Delta}=\beta \frac{k_{\mathrm{obs}, \mathrm{P}}}{R_{\mathrm{abs}}} ; \beta=\frac{k_{\mathrm{d}}^{\Delta}}{k_{\mathrm{rxn}, 1 \mathrm{O} 2}^{\mathrm{P}}}
\end{aligned}
$$

Absolute methods based on the use of a chemical probe can be classified into two groups, depending on how one determines the absolute spectral irradiance at the liquid surface $\left(I_{0, \lambda}\right)$, which is needed to calculate $R_{\mathrm{abs}}$ (various approaches described in section 3.2.1.1). A first possibility is to measure directly $I_{0, \lambda}$ by spectroradiometry. Alternatively, the absolute irradiance can be obtained via chemical actinometry.

On the other hand, relative probe-based methods rely on the use of a reference sensitizer, generally a single-molecule sensitizer, to indirectly quantify $I_{0, \lambda}$ (thus, $\Phi_{\Delta}$ ). Specifically, if DOM and a reference sensitizer are irradiated under the same light conditions, the variation in the pseudo-first-order degradation rate constants of the probe with the two sensitizers $\left(k_{\mathrm{obs}, \mathrm{P}}^{\mathrm{DOM}}\right.$ and $\left.k_{\mathrm{obs}, \mathrm{P}}^{\mathrm{Sens}}\right)$ depends only on the differences in ${ }^{1} \mathrm{O}_{2}$ quantum yields and the rates of light absorption.

$$
\begin{aligned}
& k_{\mathrm{obs}, \mathrm{P}}^{\mathrm{DOM}}=\frac{R_{\mathrm{abs}}^{\mathrm{DOM}} \Phi_{\Delta}^{\mathrm{DOM}} k_{\mathrm{rxn}, 1 \mathrm{O} 2}^{\mathrm{P}}}{k_{\mathrm{d}}^{\Delta}} \\
& k_{\mathrm{obs}, \mathrm{P}}^{\text {Sens }}=\frac{R_{\mathrm{abs}}^{\text {Sens,ref }} \Phi_{\Delta}^{\text {Sens, ref }} k_{\mathrm{rxn}, 1 \mathrm{O} 2}^{\mathrm{P}}}{k_{\mathrm{d}}^{\Delta}}
\end{aligned}
$$

By combining eqs 12 and 13 and solving for $\Phi_{\Delta}^{\mathrm{DOM}}$, one arrives at the following expression, where $R_{\mathrm{abs}}$ can be calculated with either the absolute or the normalized lamp irradiance.

$$
\Phi_{\Delta}^{\mathrm{DOM}}=\frac{k_{\mathrm{obs}, \mathrm{P}}^{\mathrm{DOM}}}{k_{\mathrm{obs}, \mathrm{P}}^{\text {Sens,ref }}} \frac{R_{\mathrm{abs}}^{\text {Sens, ref }}}{R_{\mathrm{abs}}^{\mathrm{DOM}}} \Phi_{\Delta}^{\text {Sens,ref }}
$$

Note that, when using monochromatic light (i.e., with full width at half-maximum, FWHM $<5 \mathrm{~nm}$; see section 3.2.1.5), $R_{\mathrm{abs}}^{\text {Sens,ref }} / R_{\mathrm{abs}}^{\mathrm{DOM}} \approx \varepsilon_{\lambda}^{\text {Sens,ref }}[$ Sens $] / \alpha_{\lambda}^{\mathrm{DOM}}$, where $\varepsilon_{\lambda}^{\text {Sens,ref }}\left(\right.$ in $\mathrm{L} \mathrm{mol}^{-1}$ $\mathrm{cm}^{-1}$ ) is the decadic molar extinction coefficient of the reference sensitizer, and $\alpha_{\lambda}^{\mathrm{DOM}}\left(\right.$ in $\mathrm{cm}^{-1}$ ) is the decadic absorption coefficient of the DOM solution. However, apparent quantum yield measurements are typically performed with narrow (FWHM between 5 and $50 \mathrm{~nm}$ ) and broad (FWHM >50 nm) bandwidth light sources (section 3.2.1.5); thus, the use of an integral form for $R_{\mathrm{abs}}$ remains necessary. Furthermore, if the DOM solution contains a considerable amount of suspended particles, eq 14 , and $R_{\mathrm{abs}}^{\mathrm{DOM}}$ in particular, should be readapted to account for the effect of scattering and/ or absorption from these particles (see sections 3.2.1.1 and 3.2.1.4).

3.1.2. ${ }^{1} \mathrm{O}_{2}$ Phosphorescence Methods. In ${ }^{1} \mathrm{O}_{2}$ phosphorescence experiments, singlet oxygen is directly observed via its phosphorescence emission in the near-infrared (NIR; peak at around $1270 \mathrm{~nm}) .^{87,89,109,119,120}$ This emission can be monitored either in a steady-state or in a time-resolved manner. In the first case, the photogenerated signal $S(\lambda)$ can be described as a function of wavelength according to eq $15 .{ }^{119}$

$$
S(\lambda)=\kappa k_{\mathrm{r}}^{\Delta} \tau_{\Delta} \Phi_{\Delta} R_{\mathrm{abs}}
$$

where $\kappa$ is an instrument response factor that accounts for optical efficiencies, detector response, alignment, etc., $k_{\mathrm{r}}^{\Delta}$ (in $\mathrm{s}^{-1}$ ) is the radiative relaxation rate constant of ${ }^{1} \mathrm{O}_{2}$, and $\tau_{\Delta}$ is the natural ${ }^{1} \mathrm{O}_{2}$ lifetime in the experimental media (i.e., $\tau_{\Delta}=1$ / $\left.k_{\mathrm{d}}^{\Delta}\right)$. In time-resolved measurements, $S$ will change as a function of time as indicated by eq $16 .^{119,121}$

$$
S(t)=S_{0} \frac{\tau_{\Delta}}{\tau_{\Delta}-\tau_{\mathrm{T}}}\left(e^{-t / \tau_{\Delta}}-e^{-t / \tau_{\mathrm{T}}}\right)
$$

where $\tau_{\mathrm{T}}$ is the lifetime of the triplet state of the sensitizer. The parameter $S_{0}$ relates to the overall ${ }^{1} \mathrm{O}_{2}$ signal intensity and, similar to eq 15 , is proportional to $\Phi_{\Delta}, \kappa$, and $k_{\mathrm{r}}^{\Delta}$. ${ }^{119}$

In both cases, comparing the ${ }^{1} \mathrm{O}_{2}$ signal $(S$, either its area or its amplitude) produced from DOM to a reference sensitizer irradiated under the same conditions yields eq 17 , which is analogous to the equation used in relative chemical probe determinations (eq 14). Note that, as for eq 14, the presence of particulate matter requires an appropriate treatment of $R_{\mathrm{abs}}^{\mathrm{DOM}}$ to correct for light scattering and/or absorption.

$$
\Phi_{\Delta}^{\mathrm{DOM}}=\frac{S_{\mathrm{DOM}}}{S_{\text {Sens,ref }}} \frac{R_{\mathrm{abs}}^{\text {Sens,ref }}}{R_{\mathrm{abs}}^{\mathrm{DOM}}} \Phi_{\Delta}^{\text {Sens,ref }}
$$

There are nevertheless a few differences that are worth highlighting. First, steady-state ${ }^{1} \mathrm{O}_{2}$ phosphorescence measurements have typically been performed in $\mathrm{D}_{2} \mathrm{O}$ in the presence of high DOM concentrations $\left(>100 \mathrm{mg}_{\mathrm{C}} \mathrm{L}^{-1}\right)$ in order to overcome the problems of low ${ }^{1} \mathrm{O}_{2}$ phosphorescence intensity and poor sensitivity and temporal resolution of the Ge detectors used in early studies. Due to the $\sim 20$ times longer lifetime in $\mathrm{D}_{2} \mathrm{O}$ compared to $\mathrm{H}_{2} \mathrm{O},{ }^{87,89,101}$ the use of deuterated water increases the intensity of the ${ }^{1} \mathrm{O}_{2}$ phosphorescence signal by slowing the nonradiative decay rate to ground state oxygen. ${ }^{90}$ On the other hand, this strategy comes with a side effect, as in deuterated water, physical and chemical quenching of ${ }^{1} \mathrm{O}_{2}$ by DOM also becomes relevant (because of the lower $k_{\mathrm{d}}^{\Delta}$ value in $\mathrm{D}_{2} \mathrm{O}$ compared to $\mathrm{H}_{2} \mathrm{O}$; see the Supporting Information, section S1 and Figure S1) and needs to be accounted for in quantum yield calculations. This correction can be done by determining $\tau_{\Delta}$ in the two solutions via time-resolved phosphorescence and by introducing a multiplying term $\tau_{\Delta}^{\text {Sens,ref }} / \tau_{\Delta}^{\mathrm{DOM}}$ in the apparent ${ }^{1} \mathrm{O}_{2}$ quantum 
yield formula. ${ }^{37,86,119}$ This same correction has to be accounted for in time-resolved ${ }^{1} \mathrm{O}_{2}$ phosphorescence measurements in $\mathrm{D}_{2} \mathrm{O}$ that use the area of the ${ }^{1} \mathrm{O}_{2}$ signal. ${ }^{119}$ Notably, due to advances in ${ }^{1} \mathrm{O}_{2}$ phosphorescence detection technologies over the past 30 years, time-resolved measurements can now be performed reliably in $\mathrm{H}_{2} \mathrm{O}$. Additionally, eq 17 has to be multiplied by an additional factor $t_{\text {collection }}^{\text {Sens,ref }} / t_{\text {collection }}^{\mathrm{DOM}}$ that accounts for the difference in collection times ( $\left.t_{\text {collection }}\right)$ between the reference sensitizer and DOM. ${ }^{39}$ Furthermore, one could use optically matched solutions with constant laser energies to further simplify eq 17 , as under these conditions $R_{\mathrm{abs}}^{\text {Sens,ref }} / R_{\mathrm{abs}}^{\mathrm{DOM}}{ }^{83-85}$ Lastly, one author used the slope of a phosphorescence intensity vs power plot as the ${ }^{1} \mathrm{O}_{2}$ signal $(S$ in eq 17$)^{84,98}$

\subsection{Critical Discussion of the Parameters}

In this section, we critically analyze and discuss the parameters needed for apparent ${ }^{1} \mathrm{O}_{2}$ quantum yield calculations with the aim of identifying possible sources of errors, and we provide guidelines on best practices to be considered for future studies. For the sake of simplicity, we divide the discussion into two major topics, which represent the two key components of $\Phi_{\Delta}$ determinations (eq 1): (1) Measuring light absorption (section 3.2.1) and (2) measuring singlet oxygen production (section 3.2.2). In the first section, we define equations for $R_{\mathrm{abs}}$ and we highlight problems related to the selection of the integration range (section 3.2.1.1). We then describe the challenges of obtaining absolute irradiance measurements via radiometry (section 3.2.1.2) and the working principles of chemical actinometry (section 3.2.1.3), giving an overview of the pros and cons of the methods that have been used in environmental photochemistry studies. Finally, we discuss the collection and treatment of absorption spectra (section 3.2.1.4) and the problems related to the selection of the light source (section 3.2.1.5). In the part focusing on measuring ${ }^{1} \mathrm{O}_{2}$ production, we discuss important aspects pertinent to ${ }^{1} \mathrm{O}_{2}$ probe compounds, including selection of the probe (section 3.2.2.1), data analysis protocols (section 3.2.2.2), bimolecular rate constants with ${ }^{1} \mathrm{O}_{2}$ ( $k_{\mathrm{rxn}, 102}^{\mathrm{P}}$; section 3.2.2.3), and accurate measurements of unimolecular rate constants $\left(k_{\mathrm{obs}, \mathrm{P}}\right.$; section 3.2.2.4). We next discuss the problem of selecting good reference sensitizers (section 3.2.2.5) and we present updated literature values for the ${ }^{1} \mathrm{O}_{2}$ deactivation rate constant $\left(k_{\mathrm{d}}^{\Delta}\right.$; section 3.2.2.6).

3.2.1. Measuring Light Absorption. 3.2.1.1. Rate of Light Absorption: Expressions, Approximations, and Integration Range. The rate of light absorption $\left(R_{\mathrm{abs}}\right)$ represents the moles of photons absorbed by the chromophore of interest per unit of time and volume [units of (mol absorbed photons) $\mathrm{L}^{-1} \mathrm{~s}^{-1}$; in the environmental photochemistry literature, the unit Einstein (E) is often used instead of (mol absorbed photons), even if IUPAC discourages its use $\left.{ }^{122,123}\right]$. Several authors have calculated $R_{\mathrm{abs}}$ according to eq 18 (Table S1), which directly derives from the physics of light absorption. $^{88,124}$

$$
R_{\mathrm{abs}}=\frac{1}{l} \sum_{\lambda} I_{0, \lambda}\left(1-10^{-\alpha_{\lambda} l}\right) \Delta \lambda
$$

where $I_{0, \lambda}$ (in (mol photons) $\mathrm{s}^{-1} \mathrm{~nm}^{-1} \mathrm{~cm}^{-2}$ ) is the absolute spectral irradiance at the liquid surface, $\alpha_{\lambda}\left(\right.$ in $\left.\mathrm{cm}^{-1}\right)$ is the decadic absorption coefficient of the chromophore solution, and $l$ (in $\mathrm{cm}$ ) is the optical path length. Equation 18 should be used only in the presence of a single chromophore or class of chromophores (i.e., CDOM), a typical situation when irradiating, for example, DOM isolates or whole natural waters with negligible levels of nitrate, or solutions of single-molecule sensitizers. If the solution contains more than one class of chromophores (i.e., CDOM and nitrate) and one wants to obtain $R_{\mathrm{abs}}$ for only one component $i$, eq 19 should be used instead.

$$
R_{\mathrm{abs}}^{i}=\frac{1}{l} \sum_{\lambda} I_{0, \lambda} \frac{\alpha_{\lambda}^{i}}{\alpha_{\lambda}^{\mathrm{tot}}}\left(1-10^{-\alpha_{\lambda}^{\mathrm{tot} l}}\right) \Delta \lambda
$$

where $\alpha_{\lambda}^{i}$ is the decadic absorption coefficient of component $i$, and $\alpha_{\lambda}^{\text {tot }}=\sum_{i} \alpha_{\lambda}^{i}$ is the total decadic absorption coefficient of the solution. Several authors have applied this equation to assess $R_{\mathrm{abs}}$ for DOM in whole waters with relatively high levels of nitrate, ${ }^{55,66}$ in the presence of halides, ${ }^{54}$ or in the presence of other whole water matrix components. ${ }^{31,58,63}$ Note that for $\alpha_{\lambda}^{\text {tot }}=\alpha_{\lambda}^{i}$, eq 19 simplifies to eq 18. Furthermore, both equations take into account the self-screening caused by chromophore itself and are therefore valid at any chromophore concentration. This is not the case for simplified versions that have been used in some studies (see the Supporting Information, section S2 and Table S1). A version of eq 19 should also be employed to calculate DOM's rate of light absorption in the presence of light scattering particles.

Even if there appears to be an overall literature agreement on the $R_{\mathrm{abs}}$ equations, there is less consensus on the wavelength range to be used in these calculations. This detail becomes particularly important when using polychromatic light sources, such as xenon lamps and natural sunlight. The most common approach is to integrate between 290 and $400 \mathrm{~nm}$ (Table S2) following the rationale that solar radiation below $290 \mathrm{~nm}$ does not reach the Earth surface, and that ${ }^{1} \mathrm{O}_{2}$ production is "negligible" above $400 \mathrm{~nm} .{ }^{54,56}$ Despite their apparent reasonability, these assumptions are the origin of a systematic error in final $\Phi_{\Delta}$ values. Evidence of this error was clearly shown in 2016 by McKay and co-workers, ${ }^{60}$ who performed their polychromatic apparent quantum yield calculations using three integration ranges and obtained different results in each case. For example, for SRFA, they reported $\Phi_{\Delta}=(19.0 \pm$ $0.5) \%$ for $\Delta \lambda=290-350 \mathrm{~nm},(2.13 \pm 0.06) \%$ for $\Delta \lambda=290-$ $400 \mathrm{~nm}$, and $(1.13 \pm 0.06) \%$ for $\Delta \lambda=290-450 \mathrm{~nm}$. More recently, O'Connor et al. ${ }^{78}$ obtained $\Phi_{\Delta}$ in the range of $0.7 \%$ to $3.5 \%$ using an integration range of $275-600 \mathrm{~nm}$ (solar lamp), and found that reducing the integration interval to 275-400 nm would cause a dramatic increase in apparent ${ }^{1} \mathrm{O}_{2}$ quantum yields (2.2-15.9\%). Interestingly, until 2018, the majority of the studies ( 14 of $16,88 \%$; Table S2) were still adopting the often-used limit of $400 \mathrm{~nm}$ as upper integration wavelength $\left(\lambda_{\text {up }}\right)$. In the past two years, the tendency has inverted, with more authors extending $\lambda_{\text {up }}$ up to $700 \mathrm{~nm}$ (Table S2). Note that this problem does not arise when using narrow bandwidth and single wavelength sources as far as the integration range is selected to cover the entire bandwidth.

Following on these results and motivated by the apparent confusion in the choice of $\lambda_{\text {up }}$ (Table S2), we performed a more systematic analysis to find out the minimal integration range needed to obtain reliable quantum yield values. We performed chemical probe measurements using SRFA and a xenon lamp, and we quantified the surface irradiance via both absolute and relative methods. Using the experimental rate constants, we calculated $\Phi_{\Delta}^{\mathrm{SRFA}}$ by incrementally increasing $\lambda_{\text {up }}$ to up to $800 \mathrm{~nm}$ (details in section S3). Figure 4 summarizes the results of our analyses. In all cases, $\Phi_{\Delta}$ converged to an 


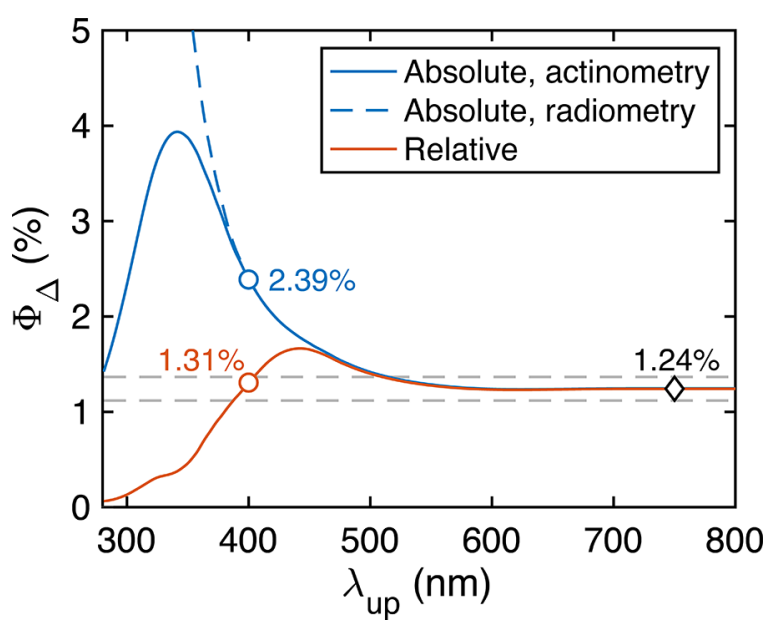

Figure 4. $\Phi_{\Delta}$ for SRFA calculated using PNA as the actinometer (continuous blue line) or PN as the reference sensitizer (continuous orange line) as a function of the upper wavelength $\left(\lambda_{\text {up }}\right)$ set in the integration range for $R_{\mathrm{abs}}$. The effect of $\lambda_{\text {up }}$ when performing radiometric irradiance measurements (dashed blue line) was estimated from the PNA data. In these calculations, the lower wavelength was set to $280 \mathrm{~nm}$. As the wavelength range used in the calculations increased, the apparent quantum yield converged to a value of $1.24 \%$ for all three methods. The gray dotted lines indicate a $\pm 10 \%$ interval of the converged quantum yield value of $1.24 \%$ (black diamond). Blue and orange circles indicate the $\Phi_{\Delta}$ obtained from the same experimental data using $\lambda_{\text {up }}=400 \mathrm{~nm}$. Details of the calculations can be found in the Supporting Information (section S3).

invariant value of $1.24 \%$ for $\lambda_{\text {up }}>580 \mathrm{~nm}$ and was within $10 \%$ of this converged value for $\lambda_{\text {up }}>500 \mathrm{~nm}$. At lower wavelengths, $\Phi_{\Delta}$ followed different trends depending on the method used to quantify the incident irradiance. When using PNA or radiometry, $\Phi_{\Delta}$ was considerably overestimated for $\lambda_{\text {up }}$ $=400 \mathrm{~nm}$ (+92\%, blue line), while, with $\mathrm{PN}, \Phi_{\Delta}$ was fortuitously within $10 \%$ of the converged value $(+6 \%$, orange line). The considerable variability in errors between the two approaches reflects differences in the absorption spectra of the reference compounds, either PNA or PN. On the other hand, the large variability as a function of $\lambda_{\text {up }}$ can only be justified by recognizing that DOM absorption at wavelengths longer than $400 \mathrm{~nm}$ is more relevant than currently believed. Thus, if we limit the integration range to $290-400 \mathrm{~nm}, R_{\mathrm{abs}}$ will be underestimated, resulting in a considerable overestimation of $\Phi_{\Delta}$. From our analysis, the minimum integration range when using a xenon lamp (and SRFA) appears to be $\Delta \lambda=290-600$ $\mathrm{nm}$, similarly to what was proposed recently by O'Connor et al. $^{78}$ However, as we discuss in section 3.3 , this range is susceptible to variations based on differences in DOM absorption spectra and concentrations. Further discussions on the effect of $\lambda_{\text {up }}$ on apparent ${ }^{1} \mathrm{O}_{2}$ quantum yields and recommendation for future studies are provided in sections 3.3 and 3.4, respectively.

3.2.1.2. Absolute Irradiance Measurements via Radiometry. Obtaining an accurate measurement of absolute spectral irradiance at the liquid surface $\left(I_{0, \lambda}\right)$ is the most critical part of absolute $\Phi_{\Delta}$ determinations. The most popular choice (25 of 49 studies) is to measure $I_{0, \lambda}$ directly at the sample position (or at the water surface) with a calibrated spectroradiometer (or a calibrated bolometer, used in early studies ${ }^{33,47}$ ). With a spectroradiometer, the incoming light is captured by input optics, which directs the light toward a detector that converts it to an electrical signal. ${ }^{125,126}$ Spectroradiometers can detect emissions in a spectrally resolved manner, while radiometers are used to measure total irradiance values (i.e., not spectrally resolved) at a single wavelength or over a wavelength range. ${ }^{126}$

However, performing an accurate irradiance measurement is more challenging than it might appear at first glance. A critical problem involves the conversion of the $I_{0, \lambda}$ value measured with the spectroradiometer outside the liquid sample to a value that represents the irradiance inside the solution (also called fluence rate), ${ }^{127}$ which is the parameter needed in photochemical calculations. Bolton and Linden ${ }^{127}$ listed all possible corrections that one has to consider when working with a Petri dish illuminated from the top with a collimated light beam (i.e., reflection due to air-water refractive index differences, spatial variations in $I_{0, \lambda}$, not perfectly collimated beam, etc.). Even if some authors adopted this setup and guidelines, ${ }^{46,48}$ the majority of the studies employed a different irradiation setup and did not provide any information on irradiance corrections (Table S3). These corrections might not considerably impact $I_{0, \lambda}$ when using dark-painted beakers irradiated from the top, but are critical when using cylindrical glassware irradiated from the side, as the in-solution irradiance can be 1.5 to 3 times higher than the value measured with the spectroradiometer due to lens and internal reflection effects. ${ }^{118,128}$

In addition, radiometers have to be recalibrated periodically (ideally, at least once a year) to provide accurate measurements. ${ }^{127}$ The radiometer head has to be carefully oriented toward the incident light, since small angular deviations can lead to distorted readings. This effect can be minimized by using a cosine corrector, an integrating sphere, or a diffuse reflectance panel. ${ }^{129}$ Other difficulties include wavelengthdependent sensitivities and variable power output from the lamp, as radiometry is typically conducted at the beginning and/or at the end of the experiment, and not for its entire duration. Taken together, all of these minor details can introduce biases and systematic errors that are hard to assess and quantify a posteriori and contribute to the variability of measured $\Phi_{\Delta}$ values.

3.2.1.3. Absolute Irradiance Measurements via Chemical Actinometry. Absolute irradiance measurement via chemical actinometry eliminates some of the drawbacks of radiometric measurements, as it provides in-solution irradiance values. In general, the absolute spectral irradiance $\left(I_{0, \lambda}\right)$ can be expressed as the product of relative irradiance $\left(I_{\lambda, \text { rel }}\right)$ and a wavelengthindependent scaling factor $(\gamma)$, which represent the (wavelength-integrated) irradiance (eq 20). ${ }^{130}$

$$
I_{0, \lambda}=\gamma I_{\lambda, \text { rel }}
$$

where $I_{\lambda, \text { rel }}$ is calculated from eq 21 using the irradiance spectrum measured with a spectroradiometer (either calibrated or uncalibrated) or the spectrum provided by the lamp manufacturer $\left(I_{\lambda, \mathrm{m}}\right){ }^{130}$

$$
I_{\lambda, \text { rel }}=\frac{I_{\lambda, \mathrm{m}}}{\sum_{\lambda} I_{\lambda, \mathrm{m}}}
$$

The scaling factor $\gamma$ is then calculated from the direct photolysis rate constant of the actinometer compound and its direct photolysis quantum yield (see below).

Two types of chemical actinometers have been used in $\Phi_{\Delta}$ studies: high optical density (OD) or low OD actinometers. ${ }^{131,132}$ High OD actinometers are highly concentrated 
solutions that absorb virtually all incident photons (solution absorbance $>2$ ), meaning that, similarly to radiometric measurements, they do not include increased irradiance due to internal reflections. The most common high OD actinometer used in $\Phi_{\Delta}$ determinations is the ferrioxalate actinometer, ${ }^{133}$ which was employed in seven publications before 2015 (Table S4). Low OD actinometers are dilute solutions (typically, solution absorbance $<0.02$ ) that can directly provide fluence rate values (i.e., incident irradiance inside the solution) if the actinometer solution is kept in the same reaction vessel as the experimental sample. The most widely used low OD actinometer in environmental photochemistry studies is the $p$ nitroanisole/pyridine system (PNA-py), ${ }^{128,130}$ which was employed in thirteen studies. In addition, one publication used $p$-nitroacetophenone/pyridine (PNAP-py), and four authors used 2-nitrobenzaldehyde (2-NBA) (Table S4). Faust and Allen ${ }^{6}$ used also valerophenone as the chemical actinometer in some of their $\Phi_{\Delta}$ measurements; due to its limited application, this compound is not included in the discussion.

In the following sections, we briefly describe the working principles of the most commonly used actinometers used in $\Phi_{\Delta}^{\mathrm{DOM}}$ measurements and the parameters needed for accurate irradiance quantification.

3.2.1.3.1. $p$-Nitroanisole/pyridine (PNA-py) and p-Nitroacetophenone/pyridine (PNAP-py). The PNA and PNAP actinometers were developed by Dulin and Mill in 1984 for the measurement of UV sunlight irradiation, ${ }^{128}$ and are the most common actinometers in environmental photochemistry. ${ }^{130,132}$ Both systems are based on a photonucleophilic aromatic substitution reaction involving the attack of pyridine (the nucleophile) on the aromatic ring of nitrobenzene derivatives (i.e., $p$-nitroanisole or $p$-nitroacetophenone). This actinometer can be used in the range 300-400 nm (UVA - UVB), where PNA(P) absorbs light (Figure 5). A clear advantage of this actinometer over 2-NBA or ferrioxalate is that the direct photolysis quantum yield can be customized by varying the initial concentration of pyridine (see eqs 23 and 24), allowing

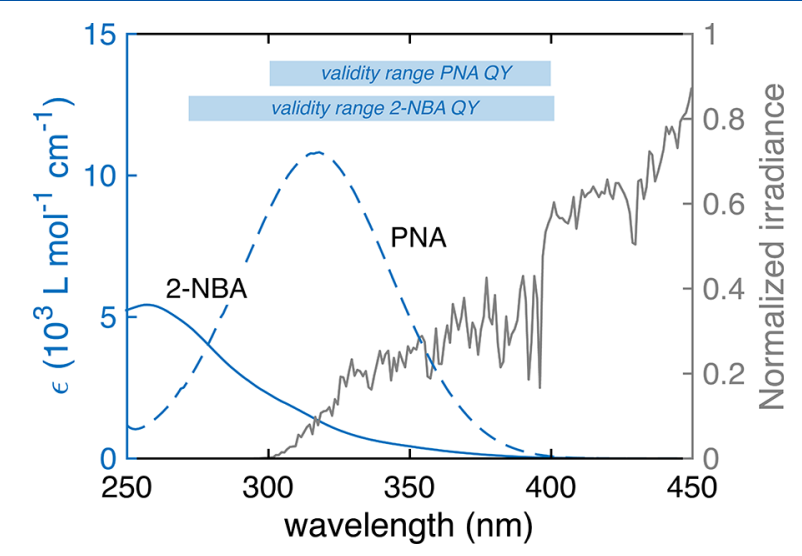

Figure 5. Comparison between literature molar extinction coefficients for PNA (dashed blue line; values from Laszakovits et al. ${ }^{130}$ ) and 2NBA (continuous blue line; values from Galbavy et al. ${ }^{134}$ ), and the normalized solar irradiance (gray; values from Appel and McNeill ${ }^{135}$ for $40{ }^{\circ} \mathrm{N}$, normalized by the maximum irradiance value in the interval 300-800 $\mathrm{nm}$ ). In the graph, we also indicate the validity range for the quantum yields according to Laszakovits et al. ${ }^{130}$ and Galbavy et al. ${ }^{134}$ for PNA and 2-NBA, respectively. one to match the time scale of the actinometry experiment to that of the ${ }^{1} \mathrm{O}_{2}$ probe.

At sufficiently high pyridine concentrations (i.e., [py]/ $[\mathrm{PNA}(\mathrm{P})]$ from 10 to 1000$), \mathrm{PNA}(\mathrm{P})$ degrades with pseudofirst-order kinetics, which allows one to calculate the scaling factor $\gamma$ via eq 22 (derivation in section S4.1). ${ }^{130}$

$$
\gamma=\frac{k_{\mathrm{obs}, \mathrm{PNA}(\mathrm{P})}[\mathrm{PNA}(\mathrm{P})]_{0} l}{\Phi_{\mathrm{dir}}^{\mathrm{PNA}(\mathrm{P})} \sum_{\lambda} I_{\lambda, \mathrm{rel}}\left(1-10^{-\varepsilon_{\lambda}^{\mathrm{PNA}(\mathrm{P})}[\mathrm{PNA}(\mathrm{P})]_{0} l}\right) \Delta \lambda}
$$

where $k_{\mathrm{obs}, \mathrm{PNA}(\mathrm{P})}$ is the $\mathrm{PNA}(\mathrm{P})$ pseudo-first-order degradation rate constant measured under the same conditions (i.e., reaction vessel and irradiation intensity) as the experimental solution, $[\mathrm{PNA}(\mathrm{P})]_{0}$ (in mol $\mathrm{L}^{-1}$ ) is the initial $\mathrm{PNA}(\mathrm{P})$ concentration, $l$ is the path length, $I_{\lambda \text {,rel }}$ is defined according to eq $21, \varepsilon_{\lambda}^{\mathrm{PNA}(\mathrm{P})}$ (in $\mathrm{L} \mathrm{mol}^{-1} \mathrm{~cm}^{-1}$ ) is the molar extinction coefficient for $\operatorname{PNA}(\mathrm{P})$, and $\Phi_{\mathrm{dir}}^{\mathrm{PNA}(\mathrm{P})}$ is the $\mathrm{PNA}(\mathrm{P})$ direct photolysis quantum yield defined according to eqs 23 and $24 .^{130}$

$$
\begin{aligned}
& \Phi_{\mathrm{dir}}^{\mathrm{PNA}}=0.29[\mathrm{py}]+2.9 \times 10^{-4} \\
& \Phi_{\mathrm{dir}}^{\mathrm{PNAP}}=\left(7.4 \times 10^{-3}\right)[\mathrm{py}]+1.1 \times 10^{-5}
\end{aligned}
$$

The direct photolysis quantum yields for PNA and PNAP are constant in the wavelength range $300-400 \mathrm{~nm},{ }^{128,130}$ and have very low temperature dependence. ${ }^{128}$ The molar extinction coefficient for PNA has recently been reassessed by Laszakovits et al., ${ }^{130}$ and it is available in the wavelength range $250-400$ $\mathrm{nm}$. It is a good practice to use purified PNA (i.e., crystallized from petroleum ether or hexane) ${ }^{136}$ for actinometry measurements, as the impurities present in the commercially available material influence the absorption coefficients of the actinometric solution (Figure S5A). If recrystallized PNA is not available, one can use eq 25 , which is a modified version of eq 22 that accounts for the additional light screening caused by impurities that might be present in the PNA stock (derivation in section S4.2).

$$
\gamma=\frac{k_{\mathrm{obs}, \mathrm{PNA}(\mathrm{P})}}{2.303 \Phi_{\mathrm{dir}}^{\mathrm{PNA}(\mathrm{P})} \sum_{\lambda} I_{\lambda, \mathrm{rel}} \varepsilon_{\lambda}^{\mathrm{PNA}(\mathrm{P}), \text { lit }} S_{\lambda}^{\mathrm{PNA}(\mathrm{P}), \mathrm{sol}} \Delta \lambda}
$$

where $S_{\lambda}^{\mathrm{PNA}(\mathrm{P}) \text {,sol }}$ is the screening factor of the $\mathrm{PNA}(\mathrm{P})$ solution used in the actinometry experiment. The screening factor is

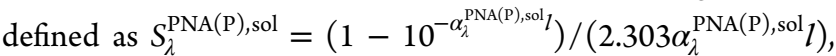
where $\alpha_{\lambda}^{\mathrm{PNA}(\mathrm{P}) \text {,sol }}$ is the molar absorption coefficient of the experimental PNA(P) solution before irradiation. Thus, in this case, one would record the actinometer solution absorbance at the beginning of the experiment instead of quantifying the initial PNA(P) concentration via HPLC. Due to light screening from pyridine (Figure S5B), one should adopt eq 25 also when using an irradiation source that extends below $300 \mathrm{~nm}$ (e.g., xenon lamp without filters, UVB bulbs; see also Figure 6).

Besides accounting for possible impurities in the PNA stock, eq 25 does not require the accurate quantification of the initial PNA(P) concentration (thus, the initial degradation rate, which is defined as $\left.k_{\text {obs,PNA(P) }}[\operatorname{PNA}(\mathrm{P})]_{0}\right)$, a quantity that is more subject to analytical errors than rate constants. Thus, even if eq 22 is the preferred one in apparent ${ }^{1} \mathrm{O}_{2}$ quantum yield determinations, we believe that eq 25 is more robust, and we therefore recommend its application in future studies. In addition, most authors (Table S5) used a simplified form of eq 22 that is valid only in low absorbance ranges. Even if they 
generally used low PNA concentrations $\left([\mathrm{PNA}]_{0} \leq 10 \mu \mathrm{mol}\right.$ $\mathrm{L}^{-1}$, resulting in $<8 \%$ error in $\gamma$; see Figure S4), we do not recommend such an approach, as it introduces unnecessary approximation errors.

All studies published before 2017 used the direct PNA photolysis quantum yields from Dulin and Mill (Table S5), which is approximately $30 \%$ larger than the value from Laszakovits and co-workers. ${ }^{128,130}$ According to a calculation performed by Maizel et al., ${ }^{76}$ the use of the PNA quantum yield value from Dulin and Mill results in an apparent $\Phi_{\Delta}$ increase of $\sim 12 \%$ (i.e., for Crystal lake, $\Phi_{\Delta}$ (Dulin and Mill)/ $\Phi_{\Delta}($ Laszakovits $\left.)=1.12\right)$, implying that apparent ${ }^{1} \mathrm{O}_{2}$ quantum yield values using this actinometry system obtained before 2017 are overestimated.

3.2.1.3.2. 2-Nitrobenzaldehyde (2-NBA). Another popular actinometer is based on the photoisomerization of 2-nitrobenzaldehyde to 2-nitrosobenzoic acid, a reaction that was first described more than a century ago by Ciamician. ${ }^{134}$ In the context of singlet oxygen quantum yield measurements, 2-NBA was employed by four authors as a low OD actinometer, mostly in atmospheric chemistry contexts (Table S4). This actinometer follows the same working principle of PNA(P)py, ${ }^{73}$ but it comes with a few limitations. The first is the high direct photolysis quantum yield $\left(\Phi_{\text {dir }}^{2-\mathrm{NBA}}=0.41 \pm 0.02\right.$ in water), ${ }^{134}$ which typically makes 2-NBA depletion kinetics considerably faster than the ${ }^{1} \mathrm{O}_{2}$ probe. Furthermore, 2nitrosobenzoic acid, the main 2-NBA photodegradation product, strongly absorbs above $300 \mathrm{~nm} .^{137}$ Thus, as the reaction proceeds, 2-NBA degradation kinetics deviate from first-order behavior due to increasing light screening caused by the accumulation of 2-nitrosobenzoic acid. To minimize this drawback, 2-NBA photodegradation is typically followed up to only $70-75 \% 2$-NBA conversion, ${ }^{134}$ which further increases the difference between the time of the actinometer depletion kinetics and the ${ }^{1} \mathrm{O}_{2}$ probe. Lastly, 2-NBA light absorption peaks at $256 \mathrm{~nm}$ and has only a small tail in the UVA (Figure 5). Therefore, it is an actinometer best suited for use with UVC and UVB light sources rather than solar light.

3.2.1.3.3. Ferrioxalate. Ferrioxalate is a high OD actinometer that can be used in the UV and visible regions (250-500 $\mathrm{nm}) .^{138}$ Since its introduction by Hatchard et al. ${ }^{133}$ in 1956 , ferrioxalate has become the reference actinometer in several fields of photochemistry, ${ }^{138}$ and has also been preferentially used in $\Phi_{\Delta}$ measurements since 2014 (Table S4). This actinometer is based on the photochemical production of $\mathrm{Fe}(\mathrm{II})$ from $\mathrm{Fe}$ (III)-oxalate. The amount of $\mathrm{Fe}(\mathrm{II})$ released in solution is quantified spectrophotometrically following complexation with $o$-phenanthroline, and is used to calculate the irradiance of the light source.

Despite the simple working principle, performing an accurate measurement requires great care in handling the reagents. For instance, a major challenge is the photochemical instability of the ferrioxalate solution, which needs to be prepared and handled under dark room conditions to limit spurious $\mathrm{Fe}$ (II) formation. ${ }^{138} \mathrm{~A}$ second issue is related to potential interferences of the $\mathrm{Fe}(\mathrm{II})$-phenanthroline assay, which might lead to up to $40 \%$ variations in reported irradiances. ${ }^{139}$ Identified interferences include $o$-phenanthroline photodegradation products formed upon exposure of the developer solution to fluorescent room light, ${ }^{139,140}$ and competing complexation of phenanthroline with $\mathrm{Fe}(\mathrm{III})$, a problem that was reported mainly when using concentrated ferrioxalate solutions (i.e., $[$ ferrioxalate $\left.]=0.15 \mathrm{~mol} \mathrm{~L}^{-1}\right){ }^{139}$
The first interference can be limited by using freshly prepared developer solutions, developer solutions stored in the dark, or by using other developing agents (such as Ferrozine), ${ }^{129}$ while the second can be overcome by adding fluoride as a $\mathrm{Fe}$ (III) complexing agent ${ }^{139}$ or by using less concentrated ferrioxalate solutions.

Besides the technical challenges, other issues make ferrioxalate nonideal for $\Phi_{\Delta}^{\mathrm{DOM}}$ studies. First, ferrioxalate is a high OD actinometer, and therefore cannot account for internal reflection effects, which can be large in curved glassware (i.e., test tubes). ${ }^{118,128}$ Furthermore, the very high quantum yield $\left(\Phi_{\text {dir }}^{\text {ferrioxalate }}>1\right)^{138}$ makes the actinometry kinetics extremely fast, which leads to the same limitations that were already discussed for 2-NBA. In addition, $\Phi_{\text {dir }}^{\text {ferrioxalate }}$ was reported to be wavelength-dependent, and has also a small dependence on the concentration. ${ }^{138,141,142}$ Following several conflicting reports, Goldstein and Rabani ${ }^{142}$ recently reassessed the quantum yield for ferrioxalate in the range 205-304 $\mathrm{nm}$, obtaining $1.48 \pm 0.02$ at $\Delta \lambda=205-240 \mathrm{~nm}$ and $1.25 \pm$ 0.02 at $\Delta \lambda=270-340 \mathrm{~nm}$, with a steep decrease between 240 and $265 \mathrm{~nm}$.

3.2.1.4. Collection of Absorption Spectra and Data Treatment. Related to the calculation of the rate of light absorption is the measurement of the absorbance of the experimental solution $\left(A_{\lambda}\right)$, which is defined according to the Beer-Lambert law (eq 26). ${ }^{143}$

$$
A_{\lambda}=[\mathrm{A}] \varepsilon_{\lambda} l
$$

where $\varepsilon_{\lambda}$ is the molar extinction coefficient (in $\mathrm{L} \mathrm{mol}^{-1} \mathrm{~cm}^{-1}$ for discrete molecules), [A] is the molar concentration of compound $\mathrm{A}$, and $l$ is the solution path length. The BeerLambert law was originally formulated for discrete molecules, but still holds true for mixture of chromophores such as CDOM. In this case, $\varepsilon_{\lambda}$ is referred to as the specific molar absorption coefficient or apparent molar absorptivity and it is reported in $\mathrm{L} \mathrm{mg}_{\mathrm{C}}^{-1} \mathrm{~m}^{-1}$ or $\mathrm{L} \mathrm{mol}_{\mathrm{C}}{ }^{-1} \mathrm{~cm}^{-1}$, with [A] expressed in $\mathrm{mg}_{\mathrm{C}} \mathrm{L}^{-1}$ or $\mathrm{mol}_{\mathrm{C}} \mathrm{L}^{-1} \cdot 31,52,54-56,58,60,63-66$ However, it is more common to report CDOM spectral features in terms of decadic absorption coefficients $\left(\alpha_{\lambda}\right.$, in $\mathrm{cm}^{-1}$ or $\mathrm{m}^{-1}$, defined as $\left.\alpha_{\lambda}=[\mathrm{A}] \varepsilon_{\lambda}\right)$, a parameter that does not require the measurement of carbon content. Note that $\alpha_{\lambda}$ is the decadic absorption coefficient, which is a factor 2.303 larger than the natural log-based coefficient $\left(a_{\lambda}\right.$, also called Napierian absorption coefficient) often used in environmental studies, particularly by oceanographers and limnologists. ${ }^{144}$ The photochemical calculations presented in this review require the use of decadic absorption coefficients; to use Napierian values, all equations must be adapted accordingly (as done by Peterson et al.; ${ }^{36}$ see also $\mathrm{Hu}$ et al. ${ }^{144}$ ). We also note that, in the past, there has been considerable ambiguity in the use of terms related to light absorption (i.e., absorptivity vs absorption coefficients) in the environmental photochemistry literature. For more details, readers should refer to $\mathrm{Hu}$ et al. ${ }^{144}$

The parameters $\alpha_{\lambda}$ and $\varepsilon_{\lambda}$ are obtained via spectrophotometric measurements. Despite being considered a simple, routine measurement, the accurate collection of UV-vis spectra is a critical step toward calculating accurate apparent ${ }^{1} \mathrm{O}_{2}$ quantum yields (more details in section 3.3). These UVvis measurements can be influenced by a series of minor experimental details that are easy to overlook and can considerably impact the calculated ${ }^{1} \mathrm{O}_{2}$ quantum yields. A critical problem, especially when measuring polychromatic $\Phi_{\Delta}$ values, is the presence of positive or negative offsets in the 
long-wavelength tail of the DOM absorption spectrum. These offsets can originate from light fluctuations within the instrument, from the use of inappropriate blanks, from (micro)bubbles that form inside a cuvette as a refrigerated sample warms to room temperature, or from inaccurate baseline correction. A simple way to overcome this issue is to correct the entire UV-vis spectrum of a sample by subtracting the average absorbance over a portion of the visible range (i.e., average between $700-800 \mathrm{~nm}$ ) to ensure that DOM absorption $>700 \mathrm{~nm}$ is zero. ${ }^{36,37,65,76,77}$ One should also screen for negative absorbance values and set them to zero, as they will artificially decrease the value of $R_{\mathrm{abs}}$. Note that the offset correction is appropriate only for relatively dilute DOM solutions (i.e., $<50-100 \mathrm{mg}_{\mathrm{C}} \mathrm{L}^{-1}$ ), as highly concentrated samples may absorb beyond $700 \mathrm{~nm}$. An alternative solution consists of computing $R_{\mathrm{abs}}$ using an empirical absorbance function (i.e., a monoexponential or a biexponential decay with the offset set to zero) instead of the experimental spectrum, as has been done with single-molecule chromophores. ${ }^{145}$ DOM absorbance in the blue and UV range can also be influenced by similar errors, but cannot be easily corrected postprocessing. If the solution contains large amounts of suspended particles (i.e., if studying ${ }^{1} \mathrm{O}_{2}$ production from humic acid-coated nanoparticles ${ }^{84}$ ), the total absorbance should be corrected for scattering. This correction can be performed directly by employing an integration sphere or indirectly by fitting the visible absorbance to a $1 / \lambda_{n}$ function and subtracting this contribution from the total absorbance. ${ }^{84,146,147}$ If using filtered, clear solutions with TOC $<20 \mathrm{mg}_{\mathrm{C}} \mathrm{L}^{-1}$, we expect this contribution to be negligible.

For these reasons, we recommend keeping the following points in mind when collecting UV-vis spectra. First, the spectra should be corrected for baseline absorption, which can be caused by additional absorption from organic residues on the cuvette walls or from the cuvette itself (when using disposable plastic cuvettes). The baseline spectrum should be collected in the exact same cuvette that will be used to record the DOM spectrum and should contain the actual buffer or reaction media, and not just nanopure water. Second, it is advisible to perform measurements in double-beam mode, using an appropriate blank as the reference. Third, one should avoid collecting spectra with absorbance values above $1-1.5$ in the spectral region of interest to calculate $R_{\mathrm{abs}}$, as, above these values, most spectrophotometers may not provide linear responses (recall that an absorbance of 1 or 2 corresponds to absorption of $90 \%$ or $99 \%$, respectively, of the incoming photons). If absorbance is greater than these values, the solution should be diluted, and the dilution factor should be considered in the calculations; alternatively, one can use a cuvette with shorter path length. ${ }^{76}$ If using values above these thresholds, one should show that the instrument's response is linear. Similarly, one should avoid using spectra below $0.02-$ 0.01 absorbance units, which are typical uncertainties of spectroscopic measurements; ${ }^{148}$ in this case, higher path length cuvettes (i.e., 5 or $10 \mathrm{~cm}$ instead of $1 \mathrm{~cm}$ ) or more concentrated solutions should be employed. Finally, it is advisible to allow the samples to equilibrate to room temperature before collecting UV-vis spectra, ${ }^{36,37}$ as (micro)bubbles forming from dissolved gases might influence the solution absorbance. In addition to all of these points, one can also record spectra in duplicate or triplicate and use the average absorbance for further data analysis. ${ }^{60}$ Averaging is particularly important to get accurate absorbance values in the visible region.

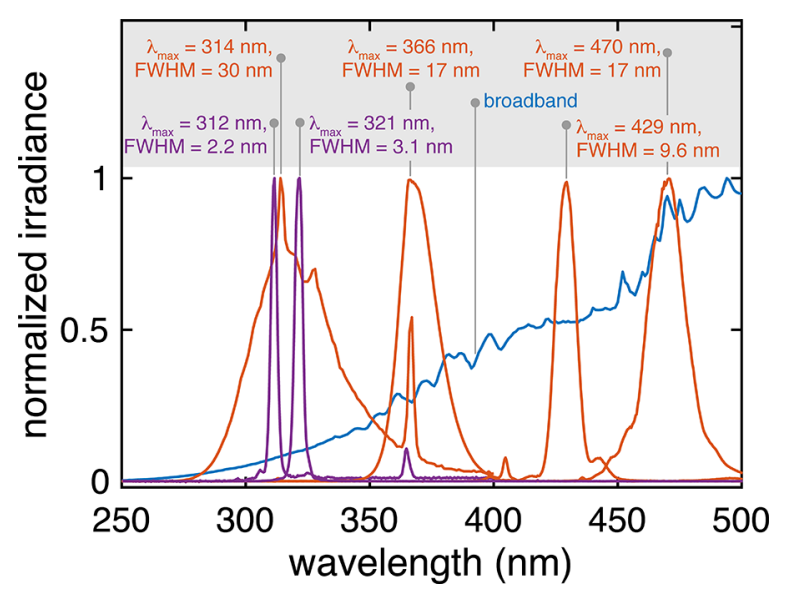

Figure 6. Example of light sources used in apparent ${ }^{1} \mathrm{O}_{2}$ quantum yield measurements (single wavelength, violet; narrow-bandwidth, orange; broad bandwidth, blue). Spectra are from Manfrin et al. ${ }^{9}$ (312 and $366 \mathrm{~nm})$, Janssen et al. ${ }^{72}(314 \mathrm{~nm})$, and Partanen et al. ${ }^{39}$ (321, 429 , and $470 \mathrm{~nm}$ ). The broadband lamp spectrum is from the xenon lamp that we used to collect the experimental data presented in this paper (Newport, $300 \mathrm{~W}$; section S5.3). This spectrum was collected with a spectroradiometer (Jaz, Ocean Optics), and here is not corrected for the absorption of the borosilicate test tube. In this graph, the normalized irradiance is defined as $I_{\lambda} / I_{\lambda_{\max }}$.

For discrete molecules, it is possible to use literature $\varepsilon_{\lambda}$ values for $R_{\mathrm{abs}}$ calculations. For example, accurate molar extinction coefficients are available for PNA in the wavelength interval $250-400 \mathrm{~nm},{ }^{130}$ which can be used directly for actinometric calculations. Spectroscopic data for a wide range of organic and inorganic species can also be downloaded from online databases such as the NIST Chemistry WebBook ${ }^{149}$ and the UV/vis ${ }^{+}$Photochemistry Database. ${ }^{150,151}$ Nevertheless, even if literature values are available, it is advisible to still collect a spectrum of the experimental solution to be able account for stock-specific impurities that might cause light screening (i.e., as when using nonrecrystallized PNA; see sections 3.2.1.3 and S4.2).

3.2.1.5. Selection of Light Sources. An important and often overlooked property of apparent ${ }^{1} \mathrm{O}_{2}$ quantum yields is its wavelength dependence. When dealing with discrete molecular chromophores, such as the reference sensitizer perinaphthenone $(\mathrm{PN}),{ }^{1} \mathrm{O}_{2}$ quantum yields are often constant over a relatively wide wavelength range because they are associated with a well-defined electronic transition. For example, irradiation of a PN solution around $360 \mathrm{~nm}$ triggers only the $S_{0} \rightarrow S_{2}$ transition, which is followed by internal conversion to $S_{1}$ and then by intersystem crossing to $T_{1}$, the first triplet excited state. ${ }^{152}$ Energy transfer from $\mathrm{T}_{1}$ to ground state oxygen results in the formation of ${ }^{1} \mathrm{O}_{2}$ with a uniform quantum yield of $\approx 1$ in water. ${ }^{152}$ In the case of chromophore mixtures like DOM, the situation is more complex, as each wavelength excites a different subset of sensitizers (even without considering further complications related to energy transfer processes). As a result, $\Phi_{\Delta}$ values of chromophore mixtures are expected to change based on the light source, both as a function of its emission maximum and its spectral bandwidth (quantified here as full width at half-maximum, FWHM). 
In the environmental photochemistry literature, three types of light sources have been used for $\Phi_{\Delta}$ measurements: single wavelength (FWHM $<5 \mathrm{~nm}$ ), narrow bandwidth (FWHM = $5-50 \mathrm{~nm}$ ), and broad bandwidth (FWHM >50 nm) sources (Figure 6).

Single wavelength sources include lasers (used most often in ${ }^{1} \mathrm{O}_{2}$ phosphorescence measurements), low-pressure $\mathrm{Hg}$ lamps (UVC; $\lambda_{\max }=253.7 \mathrm{~nm}$ ), and narrowband UVB lamps for medical applications (UVB, $\lambda_{\max }=312 \mathrm{~nm}, \mathrm{FWHM}=2.2 \mathrm{~nm}$; Figure 6), all of which have been rarely used in DOM $\Phi_{\Delta}$ determinations (Table 1). Note that laser emissions for "long" pulse-length lasers (i.e., > 100 ps) are essentially monochromatic, but there is notable broadening for short pulselength lasers (i.e., $100 \mathrm{fs}$ ), which is further exacerbated by nonlinear frequency adjustment techniques. For example, the laser data shown in Figure 6 was collected with an 80 fs pulsewidth laser system modulated with an optical parametric amplifier to produce different emission wavelengths, and shows $9.6 \mathrm{~nm}$ FWHM line width at $470 \mathrm{~nm}$.

Narrow bandwidth sources are more common, and include medium pressure mercury lamps (typically $\lambda_{\max }=366 \mathrm{~nm}$ ), black light bulbs (UVA, $\lambda_{\max }=365-366$ or $370 \mathrm{~nm}$; Figure 6), UVB light bulbs $\left(\lambda_{\max }=314 \mathrm{~nm}\right.$; Figure 6$)$, fluorescent tubes (UVA-UVB), blue light bulbs $\left(\lambda_{\max }=415 \mathrm{~nm}\right)$, filtered xenon light (various wavelengths in the UVB, UVA, and visible), and laser light modified with an optical parametric amplifier (various wavelengths in the UVB, UVA, and visible) (Table 1). Among these possibilities, several authors favored the use of black Rayonet bulbs, which emit in an environmentally relevant spectral region and have a relatively small bandwidth (9-18 nm). ${ }^{9,74-77}$ For this reason, several papers report "monochromatic" ${ }^{1} \mathrm{O}_{2}$ quantum yields at $365 \mathrm{~nm}\left(\Phi_{\Delta}(\right.$ UVA $\left.)\right)$. Note that $\Phi_{\Delta}$ values obtained with the medium pressure mercury lamps that emit at $366 \mathrm{~nm}$ are not necessarily expected to be exactly equivalent to those obtained using the black Rayonet bulbs due to possible variations in spectral shape. On the other hand, filtered xenon light (for steady-state experiments) or OPA-modified laser light (for time-resolved ${ }^{1} \mathrm{O}_{2}$ phosphorescence experiments) are the best choices to measure wavelength-dependent quantum yields, as they allow the easy manipulation of the emitted light to obtain spectral ranges that are not accessible in other commercially available lamps. For example, Halladja et al. ${ }^{70}$ used a xenon lamp equipped with a Schoeffel monochromator to obtain narrow bandwidth light at 320, 340, 375, 400, 425, 450, and $500 \mathrm{~nm}$. Likewise, Partanen and co-workers ${ }^{39}$ used a Ti:sapphire laser and an OPA to achieve high spectral resolution light (i.e., $\lambda=$ $300,320,330,340,365,380,400,430,470,500,540$, and 560 $\mathrm{nm})$.

A common broad band light source in aquatic photochemistry is the xenon lamp, which is among the most popular irradiation sources for $\Phi_{\Delta}^{\mathrm{DOM}}$ quantification via steady-state methods (Table 1, 24 publications) because it can be used to simulate natural sunlight. When used inside solar simulators, xenon lamps are typically equipped with additional filters to better reproduce the solar spectrum at the Earth's surface. Common filters are the air mass (AM) 1.5 filter, which simulates the total actinic flux at zenith angle of $48.2^{\circ}$, and cutoff (long-pass) filters, which eliminate nonenvironmentally relevant wavelengths (i.e., $<290 \mathrm{~nm}$ ) present in the xenon lamp spectrum. Note that cutoff filters can be avoided by using, for example, borosilicate test tubes instead of quartz test tubes (when irradiated from the side). A caveat of xenon lamps is that their emission, albeit similar, is not equivalent to the natural sunlight spectrum. Furthermore, the use of different of cutoff filters (see Table 1) in combination with a variety of experimental geometries (e.g., a beaker or a Petri dish irradiated from the top vs a test tube irradiated from the side; see Table S3) can influence the amount of nonenvironmentally relevant UVB wavelengths in the resulting irradiance spectrum. Even if they comprise only a minor portion of the integrated irradiance (Figure 6), wavelengths below $300 \mathrm{~nm}$ are absorbed by DOM more effectively than visible light, which might bias the final solar-integrated ${ }^{1} \mathrm{O}_{2}$ quantum yield toward higher values.

An important point to stress is that polychromatic quantum yields, like those measured with xenon lamps $\left(\Phi_{\Delta}(\right.$ solar $\left.)\right)$, will be quite different than those measured at specific wavelengths (i.e., $\left.\Phi_{\Delta}(\mathrm{UVA})\right)$. In this respect, Partanen et al. ${ }^{39}$ used their spectrally resolved $\Phi_{\Delta}$ values to compute the expected $\Phi_{\Delta}$ (solar) for SRNOM and PLFA, obtaining $1.0 \%$ and $1.9 \%$, respectively. In comparison, $\Phi_{\Delta}$ (UVA) (measured with OPAmodified Ti:sapphire laser) were $1.4 \%$ and $2.4 \%$ for SRNOM and PLFA, respectively ( $\pm 10 \%$ error), which is indeed very close but not equivalent to the respective polychromatic quantum yields. Instead, the calculated $\Phi_{\Delta}$ (solar) values matched the single-wavelength values at $430 \mathrm{~nm}$ for SRNOM and at $400 \mathrm{~nm}$ for PLFA. It is therefore important to recognize that narrow band $\Phi_{\Delta}$ values can be poor approximations of the polychromatic ${ }^{1} \mathrm{O}_{2}$ quantum yields, especially when attempting to accurately represent environmental behavior (i.e., for environmental modeling purposes). Even though this calculation was performed only for two isolates, the results from Partanen et al. suggest that blue light (i.e., blue LEDs) might be a more appropriate narrow bandwidth light source to measure apparent polychromatic quantum yields.

3.2.2. Measuring ${ }^{1} \mathrm{O}_{2}$ Production. 3.2.2.1. Choice of the ${ }^{1} \mathrm{O}_{2}$ Probe. A critical problem when performing probe-based measurements is the selection of the ${ }^{1} \mathrm{O}_{2}$ probe, which has to fulfill many criteria in order to provide robust measurements (see Nardello et al. ${ }^{153}$ ). In the environmental photochemistry field, furans have historically been used as ${ }^{1} \mathrm{O}_{2}$ probes due to their high reactivity toward this reactive oxygen species. The first authors measuring ${ }^{1} \mathrm{O}_{2}$ quantum yields in natural waters selected 2,5-dimethylfuran (2,5-DMF) as a probe because of its high bimolecular rate constant with ${ }^{1} \mathrm{O}_{2}$ and its well characterized ${ }^{1} \mathrm{O}_{2}$ degradation mechanism. ${ }^{3,67,68}$ However, already in the mid-1980s, the many limitations of 2,5-DMF - the relatively high volatility (which required the use of gastight reaction cells), ${ }^{3,68}$ the potential interference of hydrogen peroxide, and the relatively low water solubilityled to the development of new probes. ${ }^{154}$ In 1984, Haag et al. ${ }^{154}$ proposed furfuryl alcohol (FFA) as an alternative, and since then FFA has become the compound of choice for ${ }^{1} \mathrm{O}_{2}$ measurements in environmental samples, including steadystate concentrations, bimolecular rate constants, and quantum yields. ${ }^{155}$ To date, 49 studies (of 53, 92\%) have used FFA to quantify $\Phi_{\Delta}$ via absolute or relative methods (Table S6); three studies used 2,5-DMF, while one group employed furoin (1,2di(furan-2-yl)-2-hydroxyethanone). Furoin was used during the 1990s by a single research group focused mostly on the photochemistry of soil-extracted humic substances, ${ }^{69,110}$ and never gained attraction within the broader aquatic photochemistry community. 
3.2.2.2. Choice of Data Treatment Approach to Determine $R_{102}$ (Absolute Chemical Probe Methods). All absolute chemical probe methods rely on the direct or indirect quantification of the singlet oxygen formation rate $\left(R_{1 \mathrm{O} 2}\right)$, a parameter that can be obtained from experimental data by approaches based on different approximations.

The most popular approach, with at least 35 studies of 49 total (Table S7), builds on the steady-state approximation, and was already described in section 3.1.1. Briefly, if [P] is low enough not to influence ${ }^{1} \mathrm{O}_{2}$ concentration, $\left(k_{\mathrm{phys}, 1 \mathrm{O} 2}^{\mathrm{P}}+k_{\mathrm{rxn}, 1 \mathrm{O} 2}^{\mathrm{P}}\right)[\mathrm{P}]+k_{\mathrm{d}}^{\Delta} \approx k_{\mathrm{d}}^{\Delta}$, resulting in eq $6 .{ }^{29}$ This approach has different variants that were described in section 3.1.1; in all cases

$$
R_{1 \mathrm{O} 2}=\frac{k_{\mathrm{d}}^{\Delta} k_{\mathrm{obs}, \mathrm{P}}}{k_{\mathrm{rxn}, 1 \mathrm{O} 2}^{\mathrm{P}}}
$$

where $k_{\mathrm{obs}, \mathrm{P}}\left(\mathrm{s}^{-1}\right)$ is the pseudo-first-order degradation rate constant of the probe, $k_{\mathrm{rxn}, 1 \mathrm{O} 2}^{\mathrm{P}}\left(\mathrm{L} \mathrm{mol}^{-1} \mathrm{~s}^{-1}\right)$ is the bimolecular rate constant for the reaction of the probe molecule with ${ }^{1} \mathrm{O}_{2}$, and $k_{\mathrm{d}}^{\Delta}\left(\mathrm{s}^{-1}\right)$ is the aqueous singlet oxygen deactivation rate constant to ground state oxygen. In these experiments, $k_{\mathrm{obs}, \mathrm{P}}$ is obtained from the slope of a $\ln \left([\mathrm{P}] /[\mathrm{P}]_{0}\right)$ vs time plot, while $k_{\mathrm{rxn}, 1 \mathrm{O} 2}^{\mathrm{P}}$ and $k_{\mathrm{d}}^{\Delta}$ are retrieved from the literature. A major drawback of this approach is that it assumes that $\mathrm{P}$ does not influence ${ }^{1} \mathrm{O}_{2}$ steady-state concentrations. However, at typical probe concentrations, the error introduced by this approximation is well below 5\% (see section S1 and below).

On the other hand, a few authors (at least 6 studies of 49; Table S7) quantified $R_{102}$ from the initial degradation rate of the probe molecule (initial rate approach). This strategy builds on the same equations that were used for the steady-state method, but avoid any simplification. For this reason, this approach remains valid also for relatively high $[\mathrm{P}]$ (i.e., for FFA, $0.1-1 \mathrm{mmol} \mathrm{L}^{-1}$ or higher). Assuming that $-\mathrm{d}[\mathrm{P}] / \mathrm{d} t=$ $R_{\mathrm{p}}, R_{1 \mathrm{O} 2}=R_{\mathrm{abs}} \Phi_{\Delta}$, and $k_{\mathrm{phys}, 1 \mathrm{O} 2}^{\mathrm{P}}=0$, eq 4 can be rewritten as follows.

$$
R_{\mathrm{P}}=R_{1 \mathrm{O} 2} \frac{k_{\mathrm{rxn}, 1 \mathrm{O} 2}^{\mathrm{P}}[\mathrm{P}]}{k_{\mathrm{d}}^{\Delta}+k_{\mathrm{rxn}, 1 \mathrm{O} 2}^{\mathrm{P}}[\mathrm{P}]}
$$

Note that $R_{\mathrm{P}}$ will change as a function of $[\mathrm{P}]$, thus as a function of time; for this reason, we select initial concentrations and rates as the reference condition. By rearranging eq 28 , one obtains a formula for the initial ${ }^{1} \mathrm{O}_{2}$ formation rate, $R_{1 \mathrm{O} 2}^{0}$.

$$
R_{1 \mathrm{O} 2}^{0}=R_{\mathrm{P}}^{0} \frac{k_{\mathrm{d}}^{\Delta}+k_{\mathrm{rxn}, 1 \mathrm{O} 2}^{\mathrm{P}}[\mathrm{P}]_{0}}{k_{\mathrm{rxn}, 1 \mathrm{O} 2}^{\mathrm{P}}[\mathrm{P}]_{0}}
$$

Compared to eq 27, this formula relies on the accurate measurement of initial concentration and degradation rate of the probe compound, two quantities that are intrinsically more difficult to quantify accurately than rate constants. In addition, literature parameters such as $k_{\mathrm{d}}^{\Delta}$ and $k_{\mathrm{rxn}, 1 \mathrm{O} 2}^{\mathrm{P}}$ are still required for data analysis. Thus, this approach does not improve some of the limitations of the steady-state approximation.

Authors used different strategies to quantify $R_{\mathrm{p}}^{0}$. In several studies, $R_{\mathrm{P}}^{0}$ was obtained directly from the slope of a $[\mathrm{P}] /[\mathrm{P}]_{0}$ vs time plot assuming zeroth-order kinetics. ${ }^{49,52,73}$ On the other hand, Halladja et al. ${ }^{70}$ calculated $R_{\mathrm{P}}^{0}=\Delta[\mathrm{P}] / \Delta t$ using a single time point that was optimized to limit $\mathrm{P}$ degradation to $6 \%$. For these approach to work, one has to follow the probe depletion kinetics for a limited amount of time, typically
$[\mathrm{P}]_{\text {end }} /[\mathrm{P}]_{0} \approx 0.94-0.70 .{ }^{49,52,70,73}$ Alternatively, one can fit the $[\mathrm{P}] /[\mathrm{P}]_{0}$ vs time plot with a monoexponential decay function and derive the initial degradation rate as $R_{\mathrm{P}}^{0}=k_{\mathrm{obs}, \mathrm{P}}[\mathrm{P}]_{0}{ }^{38}$

We also point out that studies that are based on the steadystate approach but correct for probe quenching also indirectly quantify initial rates. For example, Du et al. ${ }^{32}$ quantified the ${ }^{1} \mathrm{O}_{2}$ production rate as $R_{1 \mathrm{O} 2}=\left[{ }^{1} \mathrm{O}_{2}\right]_{\mathrm{ss}}\left(k_{\mathrm{d}}^{\Delta}+k_{\mathrm{rxn}, 1 \mathrm{O} 2}^{\mathrm{FFA}}[\mathrm{FFA}]\right)$ using their initial FFA concentration $\left(100 \mu \mathrm{mol} \mathrm{L}{ }^{-1}\right)$. At this probe concentration, the difference between the corrected and the uncorrected $R_{102}$ value is $3.5 \%$ (Figure $S 2$ ), and it can be considered negligible. On the other hand, one author that applied the initial rate method also assumed that $k_{\mathrm{d}}^{\Delta}+$ $k_{\text {rxn,1O2}}^{\mathrm{P}}[\mathrm{P}]_{0} \approx k_{\mathrm{d}}^{\Delta}$ and obtained approximate singlet oxygen formation rates (and quantum yields) as $R_{1 \mathrm{O} 2}^{0} \approx 0.048 R_{\mathrm{P}}^{0}$, where $0.048=k_{\mathrm{rxn}, 1 \mathrm{O} 2}^{\mathrm{FFA}}[\mathrm{FFA}]_{0} / k_{\mathrm{d}}^{\Delta}$. 49

In most environmental chemistry studies, $\Phi_{\Delta}$ was quantified from a single experimental condition (typically in duplicate or triplicate). An alternative method consists of repeating the probe degradation experiment for the same sensitizer under slightly different experimental conditions (i.e., different $[\mathrm{P}]_{0}$, light intensity, sensitizer concentration, etc.) and obtaining $\Phi_{\Delta}$ from the parameters of a regression model. As the main drawback, this approach requires a considerably larger number of samples than 1-point determinations and might therefore not be always feasible. Among the studies included in this review, there is only one explicit example of such an approach. Specifically, Aguer et al. ${ }^{69}$ obtained apparent ${ }^{1} \mathrm{O}_{2}$ quantum yields of soil isolates from $\left(R_{\mathrm{P}}^{0}\right)^{-1} \mathrm{vs}[\mathrm{P}]_{0}^{-1}$ plots. For each soil sample, this plot was built by measuring initial rates at different initial probe concentrations. A linear regression of each plot provided slope and intercept, which were used to calculate the apparent ${ }^{1} \mathrm{O}_{2}$ quantum yield. Indeed, by rearranging eq 4 , one obtains the following expression:

$$
\frac{1}{R_{\mathrm{P}}^{0}}=\frac{1}{R_{\mathrm{abs}}^{0} \Phi_{\Delta}}+\frac{k_{\mathrm{d}}^{\Delta}}{R_{\mathrm{abs}}^{0} \Phi_{\Delta} k_{\mathrm{rxn}, 1 \mathrm{O} 2}^{\mathrm{P}}} \cdot \frac{1}{[\mathrm{P}]_{0}}
$$

In this study, they calculated the apparent ${ }^{1} \mathrm{O}_{2}$ quantum yield from the intercept of the plot and the rate of light absorption, a convenient choice as it requires fewer parameters than obtaining it from the slope. On the other hand, they used the ratio slope/intercept $=k_{\mathrm{d}}^{\Delta} / k_{\mathrm{rxn}, 1 \mathrm{O} 2}^{\mathrm{P}}$ (also known as $\beta$; see eq 11 ) as a quality control check. Notably, among the various approaches, this is the one that requires no reference compound and uses the fewest parameters to determine $\Phi_{\Delta}$, i.e., only the intercept and the rate of light absorption.

In addition, Kaur et al. $^{7}$ proposed a different approach based on the steady-state equations to calculate the overall apparent ${ }^{1} \mathrm{O}_{2}$ quantum yield of atmospheric samples. Specifically, they plotted $R_{102}$ (obtained via steady-state) vs $R_{\mathrm{abs}}$ for all of the samples tested and obtained the average $\Phi_{\Delta}$ directly from the slope of the regression line $\left(\Phi_{\Delta}=(3.8 \pm 0.2) \%, R^{2}=0.94, N=\right.$ $14)$. Even if this was not the strategy used in this study, this work shows that $\Phi_{\Delta}$ can be obtained from regression analysis of data from a dilution series of the same DOM sample or a series obtained using different numbers of lamps.

3.2.2.3. Bimolecular Rate Constants of the Probe with ${ }^{1} \mathrm{O}_{2}$ $\left(k_{r \times n, 102}^{P}\right)$. The bimolecular rate constant of the probe with ${ }^{1} \mathrm{O}_{2}$ $\left(k_{\mathrm{rxn}, 1 \mathrm{O} 2}^{\mathrm{P}}\right)$ is a parameter required in all absolute probe compound measurements, which are the most common $\Phi_{\Delta}$ determination techniques. In this section, we limit our discussion to FFA, which is by far the preferred ${ }^{1} \mathrm{O}_{2}$ probe in apparent ${ }^{1} \mathrm{O}_{2}$ quantum yield measurements. 
Three different bimolecular rate constants have been used to describe the reactivity of FFA with ${ }^{1} \mathrm{O}_{2}$ in $\Phi_{\Delta}^{\mathrm{DOM}}$ studies. Still today, the most used value (27 of 53, Table S6) is the one established in 1984 by Haag et al. ${ }^{154}\left(k_{\mathrm{rxn}, 1 \mathrm{O} 2}^{\mathrm{FFA}}=1.2 \times 10^{8} \mathrm{~L}\right.$ $\mathrm{mol}^{-1} \mathrm{~s}^{-1}$ ), while, in the past 15 years, a few authors (5 of 53, Table S6) have adopted the value from Latch et al. ${ }^{156}\left(k_{\mathrm{rxn}, 1 \mathrm{O} 2}^{\mathrm{FF}}\right.$ $\left.=8.3 \times 10^{7} \mathrm{~L} \mathrm{~mol}^{-1} \mathrm{~s}^{-1}\right)$, which is $40 \%$ smaller. Recently, Appiani et al. ${ }^{103}$ undertook a systematic re-evaluation of $k_{\mathrm{rxn}, 1 \mathrm{O} 2}^{\mathrm{FFA}}$ using both steady-state methods (as in Haag et al.) and timeresolved methods (as in Latch et al.) to provide a reference value for this rate constant. The benchmark value is now considered $k_{\mathrm{rxn}, 1 \mathrm{O} 2}^{\mathrm{FFA}}=1.0 \times 10^{8} \mathrm{~L} \mathrm{~mol}^{-1} \mathrm{~s}^{-1}$ at $22{ }^{\circ} \mathrm{C}$ in distilled water. ${ }^{103}$ This rate constant is not affected by $\mathrm{pH}$ over the $\mathrm{pH}$ range of $3-12$, yet it is slightly impacted by temperature $\left(\sim+2 \%\right.$ per $\left.{ }^{\circ} \mathrm{C}\right)$ and ionic strength $(+13.4 \%$ in artificial seawater). ${ }^{103}$ Appiani et al. ${ }^{103}$ also provided a rationale for the higher ${ }^{1} \mathrm{O}_{2}$ reaction rate constant measured by Haag et al. and the lower value obtained by Latch et al., further highlighting the need to use the updated, more accurate $k_{\mathrm{rxn}, 1 \mathrm{O} 2}^{\mathrm{FFA}}$ value. At this time, $66 \%$ of the 2018 publications, at least $36 \%$ of the 2019 publications, and 50\% of the 2020 publications (as of March) still use the historical Haag value (Table S6). Even if at some temperatures the difference is minimal, Wenk et al. ${ }^{66}$ conclude that the use of the updated rate constant can result in $\left[{ }^{1} \mathrm{O}_{2}\right]_{\text {ss }}$ values that are up to $24 \%$ higher compared to values obtained with the Haag's rate constant. Furthermore, a few authors have used the updated value but did not account for its temperature dependence (Table S6), which also introduces errors.

3.2.2.4. Pseudo-First Order Rate Constant of the Probe with ${ }^{1} \mathrm{O}_{2}\left(k_{\text {obs,p }}\right)$. The measurement of pseudo-first-order rate constants of the probe with ${ }^{1} \mathrm{O}_{2}\left(k_{\mathrm{obs}, \mathrm{P}}\right)$ is required for relative probe compound methods and for absolute methods that rely on the steady-state approximation. Absolute methods that utilize the initial rate approach do not strictly require the determination of this parameter, even if exception to this general rule can be found in the $\Phi_{\Delta}^{\mathrm{DOM}}$ literature (section 3.2.2.2).

In general, $k_{\mathrm{obs}, \mathrm{P}}$ is obtained empirically by irradiating a solution containing the ${ }^{1} \mathrm{O}_{2}$ probe, typically FFA, and the chromophore of interest. The loss of the probe over time is quantified via HPLC with UV-vis detection, and the plot of $\ln \left([\mathrm{P}] /[\mathrm{P}]_{0}\right)$ vs time provides $k_{\mathrm{obs}, \mathrm{P}}$ as the slope of the linear regression line. It is a good practice to follow the degradation kinetics for at least one half-life. ${ }^{71,157}$

In these experiments, the probe concentration should be low enough so that ${ }^{1} \mathrm{O}_{2}$ concentrations are not influenced by the probe itself (i.e., $k_{\mathrm{d}}^{\Delta} \gg\left(k_{\text {phys, } 1 \mathrm{O} 2}^{\mathrm{P}}+k_{\mathrm{rxn}, 1 \mathrm{O} 2}^{\mathrm{P}}\right)[\mathrm{P}]$; see eq 4$)$; alternatively, one should treat the data to account for ${ }^{1} \mathrm{O}_{2}$ losses due to reaction with P. Specifically, in order to have $<5 \%$ variation in $\left[{ }^{1} \mathrm{O}_{2}\right]_{\mathrm{ss}}$ due to probe quenching, $[\mathrm{FFA}]_{0}<145$ $\mu \mathrm{mol} \mathrm{L}^{-1},[2,5-\mathrm{DMF}]_{0}<19 \mu \mathrm{mol} \mathrm{L}{ }^{-1}$, and [furoin $]_{0}<81$ $\mu \mathrm{mol} \mathrm{L} \mathrm{L}^{-1}$ (section $\mathrm{S} 1$ and Figure S2). Overall, FFA concentrations fulfill this requirement, ranging from 1 to 100 $\mu \mathrm{mol} \mathrm{L}^{-1}$ (Table S6). To the best of our knowledge, only two studies employed $[\mathrm{FFA}]_{0}>100 \mu \mathrm{mol} \mathrm{L}^{-1}$ and did not correct for probe quenching, ${ }^{51,59}$ while three other authors used considerably higher FFA concentrations $\left(1-50 \mathrm{mmol} \mathrm{L}^{-1}\right)$ but adjusted the calculation to account for the effect of the probe on $\left[{ }^{1} \mathrm{O}_{2}\right]_{s s}{ }^{33,38,47}$ On the other hand, the three early studies that employed 2,5-DMF as the ${ }^{1} \mathrm{O}_{2}$ probe worked with concentrations from $10 \mu \mathrm{mol} \mathrm{L}-1$ to $1 \mathrm{mmol} \mathrm{L}^{-1}$ but did not account for ${ }^{1} \mathrm{O}_{2}$ quenching by the probe. ${ }^{3,67,68}$
There are a few possible additional corrections that need to be considered, including direct photolysis, dark reactions, and reactions with other PPRIs. Due to its widespread use, we will limit here the discussion to FFA. Direct photolysis is not expected to play a major role in FFA photodegradation because of the negligible absorption of FFA at wavelengths longer than $220 \mathrm{~nm} .{ }^{79}$ Nonetheless, a few authors reported measurable FFA photodegradation in their blanks, typically on the order of a few percent, and corrected $k_{\text {obs,P }}$ accordingly. ${ }^{38,63,73,76}$ We should point out that freshly distilled FFA is a colorless liquid $\left(\lambda_{\max }=219 \mathrm{~nm}\right)$, but it slowly turns yellow when exposed to air, ${ }^{158,159}$ also if stored at $4{ }^{\circ} \mathrm{C}$. Thus, the small direct photolysis observed by some authors might be the result of indirect photodegradation sensitized by the yellow impurities. Dark reactions are also negligible on the time scale of photochemistry experiments. ${ }^{6,49,59,75}$

Conversely, the reaction of FFA with other PPRIs needs to be more closely examined. A few studies have reported nonnegligible reactivity of FFA with hydroxyl radicals $(\bullet \mathrm{OH}),{ }^{9,43,46,49,55}$ which could lead to an overestimation of ${ }^{1} \mathrm{O}_{2}$-induced degradation. There is experimental evidence that this behavior is wavelength dependent, with ${ }^{\bullet} \mathrm{OH}$ contributions being significant in the UVC (up to $50 \%$ with high pressure mercury lamps), ${ }^{46}$ less important in the UVB (9-32\% with $310 \mathrm{~nm}$ lamps $)^{9}$ and negligible in the UVA (1-2\% with 365 nm lamps), ${ }^{9}$ probably as a result of the different wavelengthdependencies of ${ }^{1} \mathrm{O}_{2}$ and ${ }^{\bullet} \mathrm{OH} .{ }^{39}$ For xenon lamp photolysis, the ${ }^{\bullet} \mathrm{OH}$ contribution is estimated to be on the order of $20 \%$ of the overall FFA reactivity. ${ }^{55}$ On the other hand, some authors observed no changes in FFA degradation kinetics with the addition of ${ }^{\bullet} \mathrm{OH}$ quenchers, ${ }^{6,66,70}$ while others estimated it to be negligible based on typical PPRIs concentrations during their experiments. ${ }^{38,62}$ Overall, these results suggest that the - OH contribution to FFA loss is also DOM and sample matrix dependent (i.e., nitrate and iron content). An easy strategy to account for this problem is to amend the solution with a small amount of ${ }^{\bullet} \mathrm{OH}$ quenchers, such as methanol $\left(100 \mathrm{mmol} \mathrm{L}^{-1}\right)$, isopropyl alcohol $\left(10 \mathrm{mmol} \mathrm{L}^{-1}\right)$, or $t$-butyl alcohol $(50-100$ mmol L ${ }^{-1}$; Table S6). Alternatively, one can quantify $\left[{ }^{\bullet} \mathrm{OH}\right]_{\mathrm{ss}}$ with appropriate chemical probes (see Rosario-Ortiz and Canonica ${ }^{155}$ ) and correct $k_{\text {obs,FFA }}$ for the additional degradation due to ${ }^{\bullet} \mathrm{OH}$. Specifically, the corrected pseudo-first order rate constant can be obtained as $k_{\mathrm{obs}, \mathrm{FFA}}^{\mathrm{corr}}=k_{\mathrm{obs}, \mathrm{FFA}}-k_{\mathrm{rxn}, \mathrm{OH}}^{\mathrm{FFA}}$ $\left[{ }^{\bullet} \mathrm{OH}\right]_{\mathrm{ss}}$ where $k_{\mathrm{rxn},}^{\mathrm{FFA}}=1.5 \times 10^{10} \mathrm{~L} \mathrm{~mol}^{-1} \mathrm{~s}^{-1}$. 9,50 Other PPRIs that might induce additional non- ${ }^{1} \mathrm{O}_{2}$ degradation are triplet excited states. To date, triplet-induced FFA degradation has only been observed with anthraquinone-2-sulfonate, ${ }^{160}$ a highly oxidizing triplet excited state sensitizer $\left(E_{\mathrm{SHE}}^{0 *}=2.28 \mathrm{~V}\right.$, where $E_{\mathrm{SHE}}^{0 *}$ is the excited state reduction potential vs $\left.\mathrm{SHE}\right){ }^{5}$ Triplet excited state-mediated FFA degradation was also hypothesized to be occurring in whole water samples from Vaccarès (estuary) and Canal Fumemorte (freshwater) in Camargue, France. ${ }^{161}$ In this last study, sodium azide, a wellknown ${ }^{1} \mathrm{O}_{2}$ quencher, ${ }^{162}$ was added to the experimental solutions at a concentration that would remove $90 \%$ of the photogenerated ${ }^{1} \mathrm{O}_{2}$, but resulted in only $65 \%$ and $47 \%$ reduction in FFA reactivity for the Vaccarès and Canal Fumemorte samples, respectively. Note that similar azide quenching experiments performed by others yielded experimental results in agreement with the current understanding of FFA reactivity, ${ }^{46,70}$ suggesting that, if present, non- ${ }^{1} \mathrm{O}_{2}$ FFA degradation might be sample specific. 
Apparent ${ }^{1} \mathrm{O}_{2}$ quantum yields measurements have been performed using a wide range of DOM concentrations (0.59$150 \mathrm{mg}_{\mathrm{C}} \mathrm{L}^{-1}$, see Table S8), but this parameter should not influence dramatically the final $\Phi_{\Delta}$ value, considering that internal light screening is already accounted for if using the nonsimplified $R_{\text {abs }}$ equation (eq 18) and that ${ }^{1} \mathrm{O}_{2}$ quenching by DOM is negligible in $\mathrm{H}_{2} \mathrm{O}$ (Figure S1A).

3.2.2.5. Reference Sensitizers. Reference sensitizers are a central part of relative probe compound calculations and ${ }^{1} \mathrm{O}_{2}$ phosphorescence methods. Overall, perinaphthenone (PN) has been the most popular reference sensitizer ( 8 studies out of $12)$; other reference sensitizers that have been used in $\Phi_{\Delta}$ studies include rose Bengal (RB; 2 studies), meso-tetra( $N$ methyl-4-pyridyl)porphyrin (TMPyP; 1 study), and Suwannee River fulvic acid (SRFA; 2 studies; Table S8).

The widespread use of PN is not surprising, given that this molecule is considered a universal reference compound for determining $\Phi_{\Delta}$ in several fields of chemistry. ${ }^{163,165}$ For our purposes, a major advantage of $\mathrm{PN}$ is that its absorption spectrum resembles that of DOM (Figure 7A), which minimizes errors associated with the integration range of $R_{\mathrm{abs}}$ (section 3.2.1.1 and Figure 4). In addition, its ${ }^{1} \mathrm{O}_{2}$ quantum yield in water is well characterized and wavelengthindependent in the UVA range $(337-436 \mathrm{~nm}) .{ }^{163} \mathrm{An}$ experimental difficulty is the relatively low water solubility
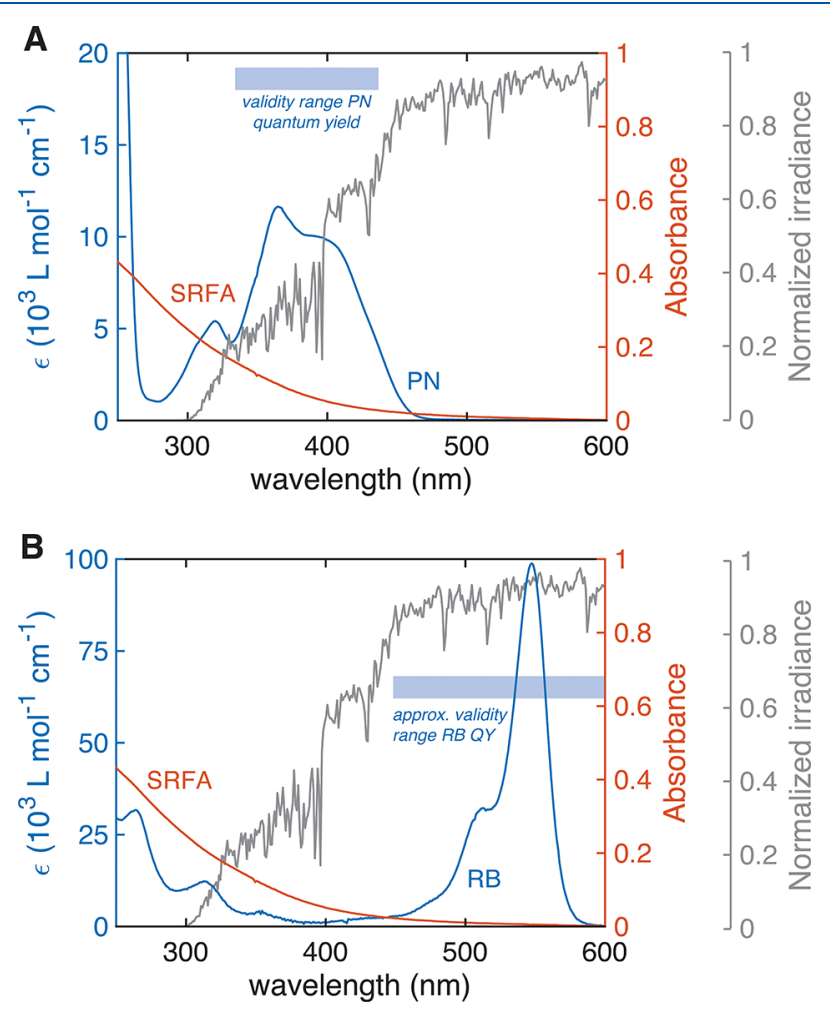

Figure 7. (A) Comparison between PN molar extinction coefficients (blue), SRFA absorption spectrum $\left(10 \mathrm{mg}_{\mathrm{C}} \mathrm{L}^{-1}\right)$, and the normalized solar irradiance (gray; values from Apell and $\mathrm{McNeill}^{135}$ for $40^{\circ} \mathrm{N}$, normalized by the maximum irradiance value). In the graph, we also indicate the validity range for the PN quantum yield value of 1.01 according to Schmidt et al. ${ }^{163}$ (B) Comparison between RB molar extinction coefficients (blue), SRFA absorption spectrum, and the normalized solar irradiance (gray). In the graph, we also indicate the approximate validity range for the $\mathrm{RB}$ quantum yield value of 0.76 by Gandin et al. ${ }^{164}$ ( $\mathrm{pH} 7$ in phosphate buffer). $\left(\sim 5 \mathrm{mg} \mathrm{L}^{-1}\right){ }^{166}$ The low solubility does not affect its use as a reference because of its high efficiency for producing ${ }^{1} \mathrm{O}_{2}$ but does make it challenging to prepare aqueous solutions. This drawback can be overcome by mixing for extended periods of time or using small amounts of a cosolvent such as ethanol or acetonitrile. Remarkably, PN has $\Phi_{\Delta}$ close to 1 in a wide range of solvents, and thus the presence of cosolvents will not alter the value of its aqueous quantum yield. ${ }^{165}$ An alternative approach to deal with PN's low water solubility that has so far not been explored in our field is to use more water-soluble versions of PN (i.e., 1-H-phenalen-1-one-2-sulfonic acid or trialkyl ammonium PN derivatives), which have dramatically higher water solubility yet still retain the excellent ${ }^{1} \mathrm{O}_{2}$ photosensitizing ability of $\mathrm{PN}\left(\Phi_{\Delta} \geq 0.97\right) .{ }^{167,168}$

The majority of the authors used a PN quantum yield of $0.98( \pm 0.08)$, but one study had $0.95 \pm 0.05$ (Table S8). Both values were reported by Schmidt et al. ${ }^{163}$ in the same article, but were obtained via different approaches: the first $(0.98 \pm$ $0.08)$ is an average of five determinations in various methanolwater mixtures, while the second $(0.95 \pm 0.05)$ is an extrapolated value recommended for any air-saturated organic solvent or $\mathrm{O}_{2}$-saturated micellar systems of low viscosity. The paper also reports a value in pure water $(1.01 \pm 0.03)$, which has never been used in relative $\Phi_{\Delta}^{\mathrm{DOM}}$ studies (besides from Sandvik and coauthors, ${ }^{37}$ who used an approximated quantum yield of 1). One author working in $\mathrm{D}_{2} \mathrm{O}^{84}$ used $\Phi_{\Delta}=0.97$ referring to Schmidt et al., but this value is not listed in the original publication. It is worth noting that all of these values are equal within their experimental error, meaning that, with appropriate error analysis, the resulting $\Phi_{\Delta}^{\mathrm{DOM}}$ values should also be statistically equivalent. Overall, all studies used PN within its applicability range, even though some used it for wavelengths below $337 \mathrm{~nm}$ (Table S8).

Other reference sensitizers-like Rose Bengal, a halogenated fluorescein derivative, and TMPyP, a water-soluble porphyrinhave been used to measure apparent ${ }^{1} \mathrm{O}_{2}$ quantum yields in the visible range, where $\mathrm{PN}$ does not absorb (Table S8 and Figure 7). Even if well suited for experiments in the visible, RB suffers a series of limitations in polar solvents, including photobleaching, aggregation, and $\mathrm{pH}$-dependent ${ }^{1} \mathrm{O}_{2}$ generation. ${ }^{165}$

A general problem when using these reference sensitizers is their disproportionately high ${ }^{1} \mathrm{O}_{2}$ quantum yield $(75-100 \%)$ compared to the value expected for DOM $(\approx 1-5 \%)$. Similar to what has been discussed for chemical actinometers, this difference makes the reference experiment considerably faster than the DOM-sensitized kinetics, even when using low reference sensitizer concentrations. Thus, the most optimal condition for $\Phi_{\Delta}^{\mathrm{DOM}}$ studies would be to use a reference sensitizer with ${ }^{1} \mathrm{O}_{2}$ quantum yields on the order of $0.5-10 \%$. Possible candidates include coumarin $\left(\Phi_{\Delta}=(1.68 \pm\right.$ $0.18) \%),{ }^{52} 7$-hydroxycoumarin $\left.\left(\Phi_{\Delta}=(1.65 \pm 0.25) \%\right)\right)^{\frac{t}{52}}$ and 6-methylcoumarin $\left(\Phi_{\Delta} \approx 1 \%\right),{ }^{169}$ as well as derivatives of 9-phenylphenalenones $\left(\Phi_{\Delta} \approx 0.2-8 \%\right),{ }^{170}$ natural products based on the perinaphthenone skeleton. ${ }^{171}$ However, a systematic reassessment of their photophysical properties (i.e., photostability, wavelength dependence, $\mathrm{pH}$, and solvent dependence) is needed before adopting them as reference sensitizers. Another alternative would be to use DOM itself as a reference sensitizer, as done by Sharpless et al. ${ }^{42}$ and Dalrymple et al. ${ }^{48}$ In these studies, the authors measured $\Phi_{\Delta}^{\text {SRFA }}$ and used it as a reference for other DOM samples measured on the exact same experimental setup. While this approach is wellsuited for discussing overall trends between DOM samples, it 
cannot be used to determine absolute values because of the lack of consensus on $\Phi_{\Delta}^{\mathrm{DOM}}$. Furthermore, Cawley et al. ${ }^{56}$ have sounded the warning that Suwannee River stocks from different sampling campaigns might have different photosensitizing abilities, as suggested by the comparison of apparent ${ }^{1} \mathrm{O}_{2}$ quantum yield values from two independent studies that employed two different SRNOM stocks (stock 2R101N: $(2.87 \pm 0.29) \%{ }^{56}$ stock $1 \mathrm{R} 101 \mathrm{~N}:(2.02 \pm$ $\left.0.23) \%^{55}\right)$. Even if variations of this order of magnitude between independent publications can easily results from other factors, as we extensively discuss in section 3.3, natural temporal fluctuations in DOM source material cannot be excluded. In addition, these organic matter samples are isolated in finite amounts, meaning that, when stocks are consumed, they can no longer be purchased (as happened recently for Pony Lake fulvic acid and other samples from the IHSS ${ }^{172}$ ). Furthermore, a good property for a reference material is that it can be purified easily, and "purification" is not well-defined for mixtures such as DOM.

3.2.2.6. Singlet Oxygen Deactivation Rate Constant $\left(k_{d}^{\Delta}\right)$. The ${ }^{1} \mathrm{O}_{2}$ deactivation rate constant in water is defined as the inverse of the natural ${ }^{1} \mathrm{O}_{2}$ lifetime (i.e., $k_{\mathrm{d}}^{\Delta}=1 / \tau_{\Delta}$ ) and is an important value needed in absolute chemical probe calculations. Several different $k_{\mathrm{d}}^{\Delta}$ values have been used in $\Phi_{\Delta}^{\mathrm{DOM}}$ determinations, ranging from 2.2 to $2.81 \times 10^{5} \mathrm{~s}^{-1}$ (Table S9). The most commonly used value is $2.5 \times 10^{5} \mathrm{~s}^{-1}$ (at least 24 papers of 48), which generally references to the work of Rodgers and Snowden, ${ }^{173}$ who were the first to measure $\tau_{\Delta}$ in $\mathrm{H}_{2} \mathrm{O}$. Rodgers and Snowden estimated two different ${ }^{1} \mathrm{O}_{2}$ lifetimes in water using either a direct measurement $\left(\tau_{\Delta}=\right.$ $4.4 \mu \mathrm{s})$ or by extrapolating $\tau_{\Delta}$ in pure water from measurements in $\mathrm{H}_{2} \mathrm{O} / \mathrm{D}_{2} \mathrm{O}$ mixtures $\left(\tau_{\Delta}=3.9 \mu \mathrm{s}\right)$. These lifetimes correspond to $k_{\mathrm{d}}^{\Delta}=2.27 \times 10^{5} \mathrm{~s}^{-1}$ and $2.56 \times 10^{5} \mathrm{~s}^{-1}$, respectively, with an average value of $2.4 \times 10^{5} \mathrm{~s}^{-1} .{ }^{173}$ This fact partially explains why authors have reported different $k_{\mathrm{d}}^{\Delta}$ values even when referencing the same paper (see Table S9). Interestingly, neither the initial work by Rodgers and Snowden $^{193}$ nor a subsequent paper by Rodgers ${ }^{194}$ report the widespread $k_{\mathrm{d}}^{\Delta}$ value of $2.5 \times 10^{5} \mathrm{~s}^{-1}$ (i.e., $\tau_{\Delta}=4 \mu \mathrm{s}$ ) in their experimental results. This value was obtained in a previous study that used an indirect photobleaching method, and it is only briefly mentioned at the end of the Rodgers and Snowden paper. ${ }^{173}$ We thus believe that the $2.5 \times 10^{5} \mathrm{~s}^{-1}$ value that is most commonly used in environmental photochemistry is the result of a rounding approximation (i.e., $3.9-4.4 \approx 4 \mu \mathrm{s}$, thus $\left.k_{\mathrm{d}}^{\Delta}=2.5 \times 10^{5} \mathrm{~s}^{-1}\right)$. We also highlight that, due to the low lifetime in $\mathrm{H}_{2} \mathrm{O}$ and the low temporal resolution and sensitivity of the experimental devices available at that time, the accurate determination of $\tau_{\Delta}$ in water was not a trivial task at the time of the Rodgers and Snowden paper. ${ }^{90,108,173}$ For these reasons, we do not recommend the use of $2.5 \times 10^{5} \mathrm{~s}^{-1}$ in future studies.

Instead, considering the advances over the past 50 years in measuring ${ }^{1} \mathrm{O}_{2}$ kinetic pathways via spectroscopy, ${ }^{90,108,109}$ we advocate for the use of a more updated $k_{\mathrm{d}}^{\Delta}$ value. Recently, Appiani et al. ${ }^{103}$ measured the singlet oxygen lifetime in water via time-resolved ${ }^{1} \mathrm{O}_{2}$ phosphorescence and found $k_{\mathrm{d}}^{\Delta}=(2.76$ $\pm 0.02) \times 10^{5} \mathrm{~s}^{-1}$ at $20{ }^{\circ} \mathrm{C}$, which corresponds to $\tau_{\Delta}=3.6 \mu \mathrm{s}$. Similarly, Davis et al. ${ }^{102}$ measured $k_{\mathrm{d}}^{\Delta}=(2.81 \pm 0.05) \times 10^{5}$ $\mathrm{s}^{-1}$ with the same experimental setup, which also gives $\tau_{\Delta}=3.6$ $\mu \mathrm{s}$. These results are in excellent agreement with the average of all the values reported in a comprehensive review $\left(\tau_{\Delta}=(3.7 \pm\right.$ $0.4) \mu \mathrm{s}),{ }^{87}$ and with recent measurements performed with a ultrafast laser setup $\left(\tau_{\Delta}=3.5 \mu \mathrm{s}\right.$ at $20{ }^{\circ} \mathrm{C}^{175}$ and $(3.5 \pm 0.1) \mu \mathrm{s}$ at $\left.25{ }^{\circ} \mathrm{C}^{101,106}\right)$. Similarly to $k_{\mathrm{rxn}, 1 \mathrm{O} 2}^{\mathrm{FF}},{ }^{1} \mathrm{O}_{2}$ lifetime does also have a weak temperature dependence. ${ }^{101,103,175}$

\subsection{Error Analysis}

In the previous section, we critically analyzed all the parameters that can be the origin of the variability of $\Phi_{\Delta}^{\mathrm{DOM}}$ values. Our analysis highlights the presence of small variations in experimental details and data analysis approaches across the environmental photochemistry literature. Considered alone, each of these factors is expected to introduce only small variations in $\Phi_{\Delta}^{\mathrm{DOM}}$, but, when considered cumulatively, they are likely to result in significant relative errors across publications. This fact also justifies the relatively good data consistency within, but not across, studies.

In Table 2, we summarize possible sources of errors related to each of the parameters discussed in the previous sections. These errors can be either experimental (i.e., improper

Table 2. Summary of Possible Sources of Errors in $\Phi_{\Delta}^{\text {DOM }}$ Studies

\begin{tabular}{|c|c|c|}
\hline parameter & possible source of error & effect $^{a}$ \\
\hline \multicolumn{3}{|c|}{ measuring light absorption } \\
\hline \multirow[t]{3}{*}{$R_{\mathrm{abs}}$} & using too narrow of an integration range & $\mathrm{L}$ \\
\hline & using simplified equation & $S$ \\
\hline & $\begin{array}{l}\text { not accounting for additional chromophores in } \\
\text { solution (i.e., nitrate) }\end{array}$ & $\mathrm{n} / \mathrm{a}$ \\
\hline \multirow[t]{4}{*}{$\begin{array}{l}I_{0, \lambda} \text { (via } \\
\text { radiometry) }\end{array}$} & $\begin{array}{l}\text { not accounting for glassware lens and reflection } \\
\text { effects }\end{array}$ & $\mathrm{n} / \mathrm{a}$ \\
\hline & improper radiometer orientation & $\mathrm{n} / \mathrm{a}$ \\
\hline & no periodic radiometer recalibration & $\mathrm{n} / \mathrm{a}$ \\
\hline & $\begin{array}{l}\text { measurement not performed for the whole } \\
\text { experiment }\end{array}$ & $\mathrm{n} / \mathrm{a}$ \\
\hline \multirow{5}{*}{$\begin{array}{l}I_{0, \lambda}(\text { via } \\
\quad \text { actinometry })\end{array}$} & using outdated $\Phi_{\mathrm{dir}}^{\text {Act }}$ value & M \\
\hline & (using nonpurified PNA) $^{b}$ & $\mathrm{n} / \mathrm{a}$ \\
\hline & using simplified equation & S \\
\hline & too fast actinometry kinetics (2-NBA, ferrioxalate) & $\mathrm{n} / \mathrm{a}$ \\
\hline & $\begin{array}{l}\text { not accounting for glassware lens and reflection } \\
\text { effects (ferrioxalate) }\end{array}$ & $\mathrm{n} / \mathrm{a}$ \\
\hline \multirow[t]{4}{*}{$\varepsilon_{\lambda}, \alpha_{\lambda}, A_{\lambda}$} & $\begin{array}{l}\text { not correcting for offsets in the long-wavelength } \\
\text { (visible) absorption }\end{array}$ & $\mathrm{L}$ \\
\hline & $\begin{array}{l}\text { improper collection of absorption spectra (i.e., } A> \\
1.5, A<0.01 \text {, no or inappropriate baseline or } \\
\text { blank collection, etc.) }\end{array}$ & $\mathrm{L}$ \\
\hline & $\begin{array}{l}\text { not accounting for presence of stock-specific } \\
\text { impurities (PNA, FFA) }\end{array}$ & $\mathrm{n} / \mathrm{a}$ \\
\hline & inaccurate quantification of optical path length & $S$ \\
\hline \multicolumn{3}{|c|}{ measuring singlet oxygen production } \\
\hline \multirow[t]{2}{*}{$k_{\mathrm{rxn}, 1 \mathrm{O} 2}^{\mathrm{P}}(\mathrm{FFA})$} & using outdated $k_{\mathrm{rxn}, 1 \mathrm{O} 2}^{\mathrm{FFA}}$ & M \\
\hline & $\begin{array}{l}\text { using updated } k_{\mathrm{rxn}, 1 \mathrm{O} 2}^{\mathrm{FFA}} \text { but not accounting for } \\
\text { variations in } \mathrm{T} \text { and ionic strength }\end{array}$ & $\mathrm{M} / \mathrm{S}$ \\
\hline \multirow[t]{2}{*}{$k_{\text {obs, } \mathrm{P}}(\mathrm{FFA})$} & using $[\mathrm{FFA}]_{0}>145 \mu \mathrm{mol} \mathrm{L}-1 c$ & $\mathrm{n} / \mathrm{a}$ \\
\hline & (not accounting for $^{\bullet} \mathrm{OH}$ reactivity) ${ }^{d}$ & $\mathrm{n} / \mathrm{a}$ \\
\hline \multirow[t]{3}{*}{$\Phi_{\Delta}^{\text {Sens,ref }}$} & using $\Phi_{\Delta}^{\text {Sens,ref }}$ outside its applicability range & $\mathrm{n} / \mathrm{a}$ \\
\hline & too fast Sens $_{\text {ref }}$ kinetics & $\mathrm{n} / \mathrm{a}$ \\
\hline & using incorrect $\Phi_{\Delta}^{\text {Sens,ref }}$ & $\mathrm{n} / \mathrm{a}$ \\
\hline$k_{\mathrm{d}}^{\Delta}$ & using outdated $k_{\mathrm{d}}^{\Delta}$ & $\mathrm{M} / \mathrm{S}$ \\
\hline
\end{tabular}

${ }^{a}$ Effect evaluated as large ( $\mathrm{L}, \geq 25 \%$ variation), medium (M, 10-25\% variation) or small (S, $\leq 10 \%$ variation) based on calculations reported in the main text for absolute actinometry-based quantum yield measurements. Note that effects of some errors were not evaluated (indicated as $\mathrm{n} / \mathrm{a}$ ). ${ }^{b}$ Without an appropriate data analysis; see eq 25 . 'When using a data analysis approach based on the steadystate approximation. ${ }^{d}$ For some DOM and light sources, this effect is negligible. 
radiometer orientation), mathematical (i.e., using too narrow of an integration range for $R_{\mathrm{abs}}$ ), or can result from the use of outdated literature parameters (i.e., using $\Phi_{\mathrm{dir}}^{\mathrm{PNA}}$ from Dulin and Mill). The first type of errors can hardly be corrected a posteriori and should be minimized as much as possible at the time of data collection. Mathematical errors can only be corrected if the entire raw data set, including lamp and DOM spectra, is available, which is typically not the case. Literature errors are the only ones that can be corrected a posteriori, but, again, only if the values of the parameters used during data analysis are reported in the manuscript.

In a first attempt to evaluate the effect of errors in literature parameters, we harmonized the available ${ }^{1} \mathrm{O}_{2}$ quantum yield values for Suwannee River fulvic acid with the most updated values for $k_{\mathrm{rxn}, 1 \mathrm{O} 2}^{\mathrm{FFA}}, k_{\mathrm{d}}^{\Delta}, \Phi_{\Delta}^{\mathrm{Sens}}$, and $\Phi_{\mathrm{dir}}^{\mathrm{PNA}}$ (Table 3). In this

Table 3. Average $\Phi_{\Delta}$ ( \pm Standard Deviation) for SRFA before and after Harmonization ${ }^{a}$

$\begin{array}{ccccc}\text { light source } & N & \Phi_{\Delta} \text { (orig) (\%) } & \Phi_{\Delta} \text { (corr) }(\%) & \Phi_{\Delta} \text { (corr)* (\%) } \\ \text { all } & 25 & 1.96 \pm 1.07 & 2.14 \pm 1.03 & 1.68 \pm 0.89 \\ & & (55 \%) & (48 \%) & (53 \%) \\ \text { solar } & 11 & 2.00 \pm 0.76 & 2.45 \pm 1.00 & \mathbf{1 . 3 9} \pm \mathbf{0 . 6 0} \\ & & (38 \%) & (41 \%) & (\mathbf{4 3} \%) \\ \text { blue } & 1 & 0.57 & \mathbf{0 . 5 7} & \\ \text { UVA } & 10 & 1.44 \pm 0.44 & \mathbf{1 . 5 5} \pm \mathbf{0 . 5 3} & \\ & & (31 \%) & (\mathbf{3 4 \%} \% & \\ \text { UVB } & 2 & 4.40 \pm 1.41 & \mathbf{3 . 2 9} \pm \mathbf{0 . 3 1} & \\ & & (32 \%) & (\mathbf{9 \%}) & \\ \text { UVC } & 1 & 3.20 & \mathbf{3 . 9 9} & \end{array}$

${ }^{a_{T}}$ The value in parentheses is the relative error calculated as the standard deviation divided by the average. $\Phi_{\Delta}($ corr $) *$ is the average of the harmonized values corrected for the upper integration range (Table S10). Note that $\Phi_{\Delta}(\text { corr })^{*}$ should be considered only indicative (see section S5.1 for details). Bold data are best values. Data for SRNOM, SRHA, and PLFA are provided in Table S11.

analysis, we included $\Phi_{\Delta}$ values measured via absolute chemical probe methods (actinometry, $N=7$; radiometry, $N$ $=13)$, relative chemical probe methods $(N=3)$, and ${ }^{1} \mathrm{O}_{2}$ phosphorescence methods $(N=2$; Figure $8 \mathrm{~A}$ and Table S10). To harmonize the literature data, we multiplied or divided the original $\Phi_{\Delta}$ value by correction factors (one for each literature parameter) obtained as the ratio of the updated value (i.e., $\left.k_{\mathrm{rxn}, 1 \mathrm{O} 2}^{\mathrm{FFA}}(\mathrm{upd})\right)$ to the value used in each publication (i.e., $k_{\mathrm{rxn}, 1 \mathrm{O} 2}^{\mathrm{FFA}}($ orig)). For instance, for the absolute probe compound method based on actinometry, we used the following equation.

$$
\Phi_{\Delta}(\text { corr })=\Phi_{\Delta}(\text { orig }) \cdot \frac{k_{\mathrm{rxn}, 1 \mathrm{O} 2}^{\mathrm{FFA}}(\text { orig })}{k_{\mathrm{rxn}, 1 \mathrm{O} 2}^{\mathrm{FFA}}(\text { upd })} \cdot \frac{\Phi_{\mathrm{dir}}^{\mathrm{PNA}}(\text { upd })}{\Phi_{\text {dir }}^{\mathrm{PNA}}(\text { orig })} \cdot \frac{k_{\mathrm{d}}^{\Delta} \text { (upd) }}{k_{\mathrm{d}}^{\Delta} \text { (orig) }}
$$

where $k_{\mathrm{rxn}, 1 \mathrm{O} 2}^{\mathrm{FFA}}$ (upd) changes as a function of temperature, and $\Phi_{\text {dir }}^{\mathrm{PNA}}$ (upd) varies as a function of pyridine concentration. When the experimental temperature was not available, it was assumed to be $25^{\circ} \mathrm{C}$. If $[\mathrm{py}]_{0}$ was not available and $\Phi_{\mathrm{dir}}^{\mathrm{PNA}}$ (orig) was from Dulin and Mill, $\Phi_{\mathrm{dir}}^{\mathrm{PNA}}($ upd $) / \Phi_{\mathrm{dir}}^{\mathrm{PNA}}($ orig $) \approx 0.29 / 0.44$ $=0.66$. If the parameters used in the original publication were not available, we assigned most likely values based on the publication year or based on values used by the same authors in other studies (Table S10). We employed analogous equations to harmonize the quantum yield values obtained with other methods (section S5.1).

Figure $8 \mathrm{~A}$ provides an overview of the effects of harmonization. Overall, we observed the most substantial effects for absolute probe compound methods, which is a direct consequence of the fact that more literature parameters are needed to calculate the quantum yield. Typically, the correction resulted in a slight increase in $\Phi_{\Delta}$, which directly stems from the use of $k_{\mathrm{rxn}, 1 \mathrm{O} 2}^{\mathrm{FFA}}$ from Haag et al. ${ }^{154}$ and $k_{\mathrm{d}}^{\Delta}=2.5$ $\times 10^{5} \mathrm{~s}^{-1}$ in most publications (Table S10). Considerable is the reduction for the Janssen et al. ${ }^{72}$ value, which dropped from $5.4 \%$ to $3.1 \%$, thus becoming comparable to the other quantum yield measured with UVB lamps (3.5\%). ${ }^{9}$ Note also that this correction reduced the spread of literature values for SRFA, which now ranges from $0.46 \%$ to $4.0 \%$. Typically, relative methods are characterized by a smaller variability associated with literature parameters, as they only rely on the ${ }^{1} \mathrm{O}_{2}$ quantum yield of the model sensitizer. This parameter is typically well constrained (i.e., for $\mathrm{PN}, \Phi_{\Delta}=0.95-1.01$; section 3.2.2.5) and scales linearly with $\Phi_{\Delta}$ (eqs 14 and 17), resulting in a maximum relative variability of $\pm 6 \%$.

Even if harmonizing apparent ${ }^{1} \mathrm{O}_{2}$ quantum yields values for the variation in literature parameters does eliminate some variability, it still does not entirely solve the problem. Indeed, the average ${ }^{1} \mathrm{O}_{2}$ quantum yield for SRFA before harmonization is equivalent to the one after correction (original: (1.96 \pm $1.07) \%$; harmonized: $(2.14 \pm 1.03) \%$; Table 3$)$, as well as the
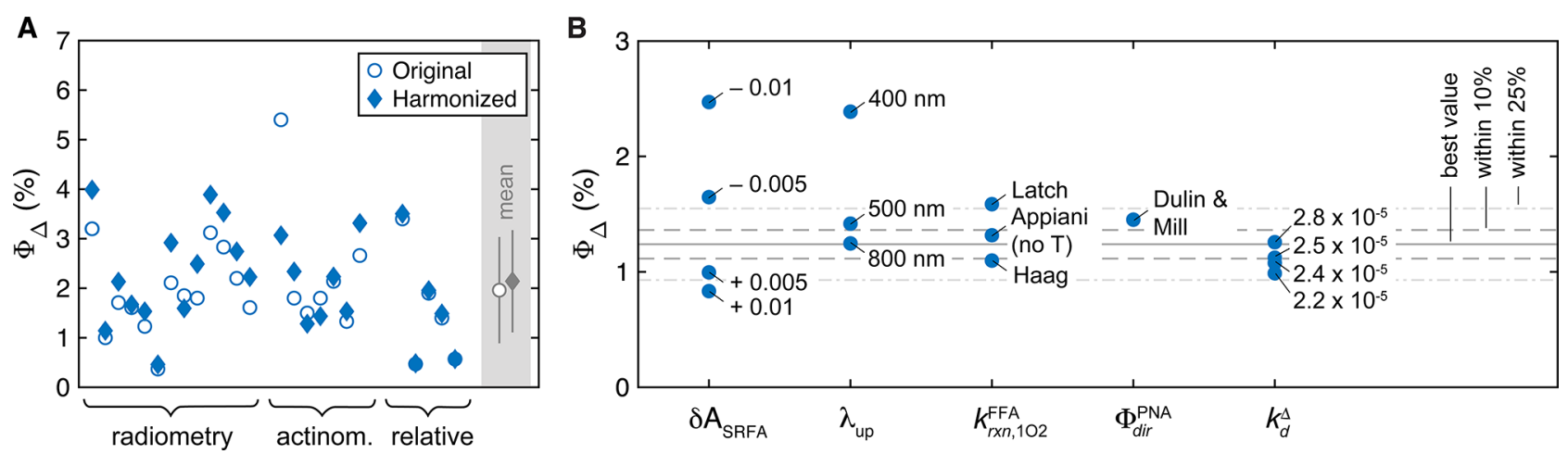

Figure 8. (A) Effect of literature parameter harmonization on $\Phi_{\Delta}$ for SRFA classified by measurement technique. Numerical values and details of the harmonization procedure are provided in Table S10 and section S5.1, respectively. (B) Analysis of most relevant parameters responsible for errors in $\Phi_{\Delta}$ values in absolute quantum determinations (actinometry). The continuuous gray line represents $\Phi_{\Delta}$ for SRFA obtained using the most updated values and an upper integration range of $650 \mathrm{~nm}\left(\Phi_{\Delta}=1.24 \%\right)$. The dashed gray line indicate the range within $10 \%$ of the best value $(1.12-1.36 \%)$, while the dash-dot line is the $25 \%$ range $(0.93-1.55 \%) . \delta \mathrm{A}_{\mathrm{SRFA}}$ indicate a variation across the entire absorbance spectrum of SRFA. 
associated relative error (original: $\pm 55 \%$; harmonized: \pm $48 \%$ ). The harmonization procedure yielded comparable results for Suwannee River NOM, humic acid, and for Pony Lake fulvic acid (Table S11). These results suggest that other factors are responsible for a larger fraction of variability in $\Phi_{\Delta}$.

To better constrain possible sources of errors, we measured the apparent ${ }^{1} \mathrm{O}_{2}$ quantum yield of SRFA using a xenon lamp and PNA as the chemical actinometer (experimental details in section S5.3). We first worked up the raw data using the most updated literature parameters for $k_{\mathrm{rxn}, 1 \mathrm{O} 2}^{\mathrm{FFA}}, k_{\mathrm{d}}^{\Delta}$, and $\Phi_{\mathrm{dir}}^{\mathrm{PNA}}$ (Table $4)$, and an upper integration range of $650 \mathrm{~nm}$. From this analysis, we obtained $\Phi_{\Delta}=1.24 \%$, in agreement with the values reported in Table 3 (i.e., $(1.39 \pm 0.60) \%)$. We then repeated the calculation varying one parameter at a time to simulate the effect of some of the errors listed in Table 2 on the final quantum yield value. Within this list, we identified eleven possible errors that could be reliably evaluated at the data analysis stage with our experimental data (details in section S5.4).

Figure $8 \mathrm{~B}$ reports the five most relevant parameters that influence ${ }^{1} \mathrm{O}_{2}$ quantum yield values ranked from the most to the least relevant. By far, $R_{\mathrm{abs}}$ appears as the most sensitive variable in apparent quantum yield determinations. In section 3.2.1.1, we have already highlighted the effect of the upper wavelength $\left(\lambda_{\text {up }}\right)$ used in $R_{\mathrm{abs}}$ calculations, which can result in a quantum yield overestimation of up to $92 \%$ for SRFA ( $\lambda_{\text {up }}=$ $400 \mathrm{~nm}$; Figure 4). The exact magnitude of the error depends on the DOM absorption spectrum (Figure S6A), and it is likely a major cause for the overall high solar-integrated $\Phi_{\Delta}$ values reported in absolute probe compound determinations (see also Table 3). Indeed, correction of literature $\Phi_{\Delta}^{\mathrm{SRFA}}$ values for differences in the upper integration wavelength resulted in a considerable decrease in the average quantum yield (i.e., from $(2.45 \pm 1.00) \%$ to $(1.39 \pm 0.60) \%$; see column $\Phi_{\Delta}($ corr $) *$ in Table 3). Similar corrections (i.e., harmonization and correction for $\lambda_{\text {up }}$ ) were performed on available literature values for PLFA, SRNOM, and SRHA, and also resulted in a considerable decrease of solar-integrated $\Phi_{\Delta}$ (section S5.2 and Table S11). It is worth noting that corrected (solar-integrated) $\Phi_{\Delta}$ values are comparable to solar-integrated ${ }^{1} \mathrm{O}_{2}$ quantum yields calculated by Partanen et al. ${ }^{39}$ using polychromatic quantum yields obtained via ${ }^{1} \mathrm{O}_{2}$ phosphorescence measurement. Specifically, for PLFA, the calculated quantum yield from Partanen et al. was $1.9 \%, \Phi_{\Delta}$ (orig) was $(2.98 \pm 1.62) \%$, and $\Phi_{\Delta}$ (corr)* was $(1.86 \pm 1.06) \%(N=9)$, while, for SRNOM, the calculated value from Partanen et al. was $1.0 \%$, $\Phi_{\Delta}$ (orig) was $(2.01 \pm 0.87) \%$, and $\Phi_{\Delta}$ (corr)* was $(1.17 \pm$ $0.50) \%(N=14)$. In addition, for the four isolates, apparent ${ }^{1} \mathrm{O}_{2}$ quantum yields followed the order $\Phi_{\Delta}(\mathrm{UVC})>\Phi_{\Delta}(\mathrm{UVB})$ $>\Phi_{\Delta}($ UVA $)>\Phi_{\Delta}($ solar $) \approx \Phi_{\Delta}$ (blue) only after harmonization and correction for $\lambda_{\text {up }}$ (Table 3 and S11), which is also expected based on known wavelength dependencies for these two isolates (section 5.4). ${ }^{39,71}$ Before correction for $\lambda_{\text {up' }}$ $\Phi_{\Delta}$ (solar) $\gg \Phi_{\Delta}$ (UVA), which is not expected based on the theoretical calculation of Partanen et al. ${ }^{39}$ and further strengthens our point that most of the solar-integrated values in the literature are inflated.

Besides the upper integration wavelength, we found that small variations in the DOM absorption spectrum also result in significant effects on the final quantum yield. To evaluate the effect of this parameter, we added a positive and a negative offset $\left(\delta \mathrm{A}_{\mathrm{SRFA}}\right)$ of 0.01 and 0.005 absorbance units across the entire SRFA spectrum (Figure 9A). This calculation simulates
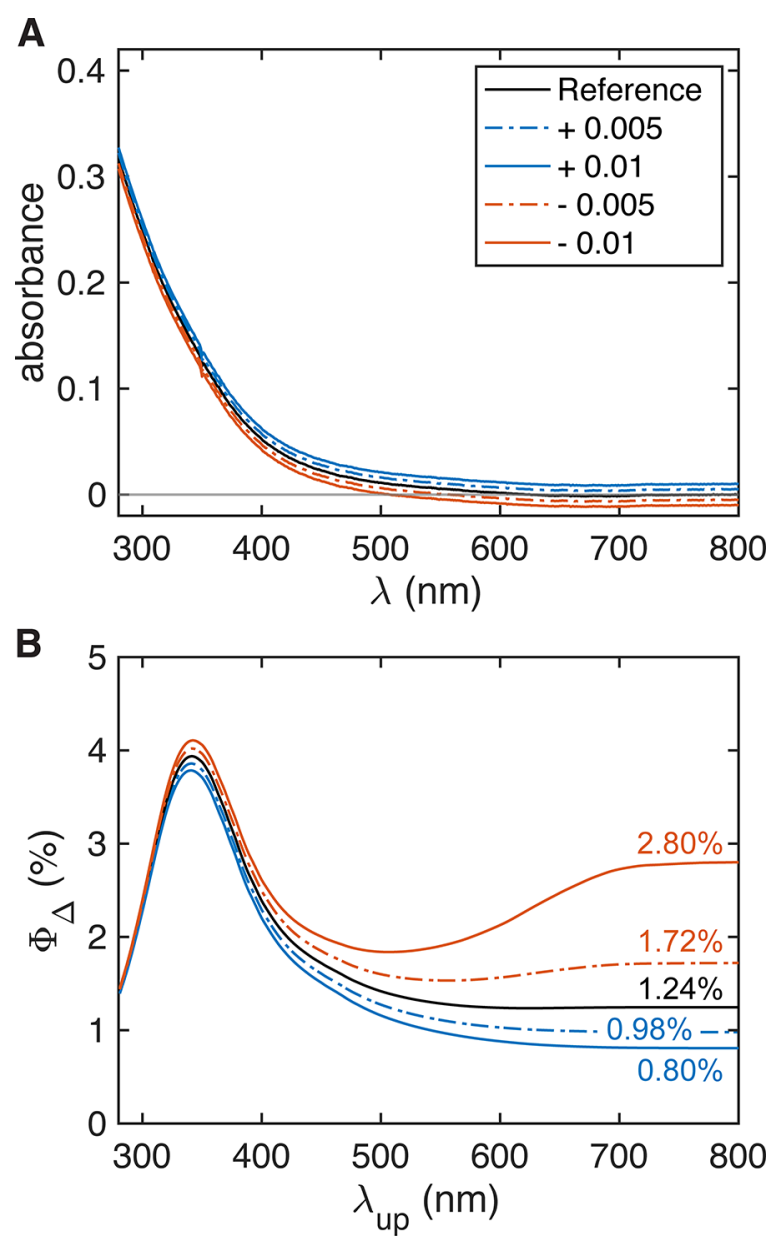

Figure 9. (A) Simulated variation in the absorption spectrum of Suwannee River fulvic acid $\left(\sim 10 \mathrm{mg}_{\mathrm{C}} \mathrm{L}^{-1}\right)$. (B) Resulting variation in apparent ${ }^{1} \mathrm{O}_{2}$ quantum yield relative to the absorption spectra in (A) as a function of upper integration range wavelength. The numerical values are apparent ${ }^{1} \mathrm{O}_{2}$ quantum yields at $\lambda_{\text {up }}=800 \mathrm{~nm}$.

common errors in the measurement of absorption spectra that can result from inaccurate baseline correction, scratched or contaminated cuvettes, or an inappropriate correction for longwavelength absorption. Note also that, for a concentration of approximately $10 \mathrm{mg}_{\mathrm{C}} \mathrm{L}^{-1}$ of SRFA, these variations are equivalent to $\pm 2 \%( \pm 0.005$ absorbance unit $)$ and $\pm 4 \%( \pm 0.01$ absorbance unit) of the absorbance at $300 \mathrm{~nm}$ and are thus easy to overlook. The cause of the substantial effect in $\Phi_{\Delta}$ (i.e., from $+99 \%$ to $-33 \%$; Figure $8 \mathrm{~B}$ ) stems directly from the fact that xenon lamp (and solar) emission is large where DOM absorption is small or zero. As a result, small variations in longwavelength DOM absorption get amplified in $R_{\mathrm{abs}}$ calculations. Consistently with this explanation, in the presence of offsets in the DOM absorption spectrum, errors in the final ${ }^{1} \mathrm{O}_{2}$ quantum yield are proportional to $\lambda_{\text {up }}$ (Figure 9B). This fact is particularly evident in the presence of negative offsets (orange lines in Figure 9B), as the error increases with $\lambda_{\text {up }}$, reaching values of $+125 \%$ of the converged quantum yield $\left(\lambda_{\text {up }}\right.$ $=800 \mathrm{~nm}$ ).

Even if not evaluated directly, the use of noncrystallized PNA without accounting for the effect of impurities on $R_{\mathrm{abs}}$ is expected to result in comparable relative errors as it also involves the accurate quantification of the solution absorbance. Likewise, errors in the determination of the incident irradiance 
in the absolute method based on radiometry can also result in small to large variations in the final ${ }^{1} \mathrm{O}_{2}$ quantum yield value (section S5.5 and Figure S8).

Errors in literature parameters contribute to small (within $10 \%$ ) or medium (within 25\%) variations in apparent ${ }^{1} \mathrm{O}_{2}$ quantum yields, and only in one case resulted in an overestimation larger than $25 \%$ of the actual value (Figure $8 \mathrm{~B})$. Note that these errors can be both positive and negative, meaning that a fortuitous combination of the wrong parameters might result in an accurate result. All other parameters analyzed resulted in small errors in the final quantum yield value (Figure S7). In particular, the use of a simplified equation for $R_{\mathrm{abs}}$ calculation resulted in negligible variations in the final output $(-2 \%)$. However, this approximation can result in more relevant errors when employing higher PNA concentrations (Figure S4). Empirical parameters such as $[\mathrm{py}]_{0},[\mathrm{PNA}]_{0}, k_{\mathrm{obs}, \mathrm{FFA}}$ and $k_{\mathrm{obs}, \mathrm{PNA}}$ are typically characterized by small relative errors (i.e., normally $\leq 5 \%)$ and scale linearly with $\Phi_{\Delta}$. Relative errors to up to $20 \%$ in the path length have negligible effects on $\Phi_{\Delta}$ when using actinometry because they propagate in the $R_{\mathrm{abs}}$ calculation for both PNA and DOM. Interestingly, the same minimal effect is observed also when the incident irradiance is quantified via radiometry (Figure S8). In this case, errors in path length cancel out as $l$ appears both on the numerator and the denominator of the $R_{\mathrm{abs}}$ expression (eq 18).

We want to stress further that our conclusions strictly apply only to actinometry-based determinations of apparent ${ }^{1} \mathrm{O}_{2}$ quantum yields of SRFA under our experimental conditions. These conditions were selected to be representative of typical absolute quantum yield determinations. However, experimental variables such as solution $\mathrm{pH}$, lamp intensity and spectral range, concentrations of PNA, pyridine, FFA, and DOM, and DOM type might influence the quantitative results presented above, resulting in slightly larger or smaller effects. Thus, these conclusions should be considered only semiquantitative trends valid for solar integrated quantum yield measurements.

Similar analyses conducted with the same approach for radiometry-based and relative probe compound methods also indicated that $R_{\mathrm{abs}}$ is the most sensitive parameter in these other approaches (sections S5.5 and S5.6). This result further strengthens the conclusion that accurate $\Phi_{\Delta}$ determination rely on precise and accurate measurements of both absorption spectra and absolute spectral irradiance, and justify the spread in literature values for SRFA also after harmonization (Figure $8 \mathrm{~A}$ ) and correction for $\lambda_{\text {up }}$ (Table 3). Overall, the relative method appears as the most robust approach among the three techniques investigated, both because it relies on a limited number of parameters that are typically characterized by small errors (i.e., $k_{\mathrm{obs}, \mathrm{FFA}}^{\mathrm{DOM}}, k_{\mathrm{obs}, \mathrm{FFA},}^{\mathrm{Sen}, \mathrm{ref}}$ and $\Phi_{\Delta}^{\text {Sens,ref }}$ ) and because of the fortuitous insensitivity of $\Phi_{\Delta}$ toward $\lambda_{\text {up }}$ (section S5.6).

\subsection{Experimental Recommendations and Research Gaps}

In this section, we present our recommendations for minimizing errors in $\Phi_{\Delta}$ measurements. It is worth noting that these recommendations, while specific to ${ }^{1} \mathrm{O}_{2}$ and DOM, are also useful when considering quantum yield determinations in other systems and for other processes, such as quantum yields for the production of other PPRIs, for the production of DOM photodegradation products, or for direct photochemical transformations of micropollutants.

A first key aspect of apparent quantum yield measurements is the selection of the light source. Being a complex mixture of organic molecules, apparent quantum yields depend not only on the photophysical features of DOM, but also on the bandwidth and spectral emission region of the lamp. Overall, the selection of the light source should be motivated by the general objective of the study. Broadband sources can be appropriate to determine solar-integrated $\Phi_{\Delta}$ values for modeling purposes (in the near-surface only; for depthintegrated simulation, wavelength-dependent quantum yields are needed $\left.{ }^{73}\right)$. However, depending on the lamp filters and the design of the experimental setup, the results might not represent the "true" solar-integrated value due to spectral differences between real sunlight and the simulator. An alternative approach is to determine the spectral dependence with single-wavelength or narrow band light sources and use this data to calculate the solar-integrated quantum yield. Despite being more accurate, this strategy is time-consuming and requires more material. Recent work by Partanen et al. ${ }^{39}$ showed that apparent ${ }^{1} \mathrm{O}_{2}$ quantum yields measured at $400-$ $430 \mathrm{~nm}$ reproduce well the calculated solar-integrated polychromatic values, indicating that blue light LEDs or fluorescent lamps can be valid surrogates of the xenon lamp. However, further work needs to confirm that this trend is valid in general and not only for SRNOM and PLFA. On the other hand, narrow band UVA light sources appear the most convenient choice for comparison purposes or for studies of fundamental photophysical properties of DOM.

Going beyond the light source, our analysis leads us to recommend the use of relative methods (i.e., those that use reference sensitizers) for future measurements of apparent ${ }^{1} \mathrm{O}_{2}$ quantum yields of DOM and atmospheric samples. Relative methods can either be probe-based or ${ }^{1} \mathrm{O}_{2}$ phosphorescencebased; however, due to the lower requirements in terms of equipment and technical expertise, we anticipate that most researchers will favor the use of relative probe-based methods. In both of these cases, the amount of singlet oxygen produced by DOM is compared to the amount produced by a calibrated, well-defined sensitizer like PN. By comparison, absolute methods, which rely on radiometry or actinometry, require more parameters, and can therefore lead to larger relative errors. Similar to the other approaches, the critical step remains the accurate determination of the solution absorbance. The main caveat of the relative probe compound method with PN as the reference sensitizer is the high ${ }^{1} \mathrm{O}_{2}$ quantum yield of $\mathrm{PN}$, which makes the reference sensitizer kinetics considerably faster compared to DOM. In addition, $\mathrm{PN}$ is a suitable sensitizer only in the UVA and blue range. To overcome these limitations, there is a need for single-molecule reference sensitizers with $\Phi_{\Delta}$ on the order of $0.5-10 \%$ that can cover the entire UVB-visible range. A well-accepted and tested list of reference sensitizers for environmental photochemistry applications has not yet been developed. Possible candidates might include coumarins and 9-phenylphenalenones. ${ }^{52,169,170}$ However, a thorough and comprehensive assessment of their properties (i.e., photochemical stability, $\mathrm{pH}$ dependence, water solubility, reactivity with other PPRIs, etc.) is required before the broad adoption of new reference sensitizers for $\Phi_{\Delta}^{\mathrm{DOM}}$ measurements. This step is critical to avoid creating reproducibility issues also in future literature.

In addition, we argue that a quality control step should be incorporated in $\Phi_{\Delta}^{\mathrm{DOM}}$ measurement protocols. A possibility is to perform a ${ }^{1} \mathrm{O}_{2}$ quantum yield measurement of a singlemolecule sensitizer under the chosen experimental condition and compare the result to the published literature. For 
Table 4. Summary of Recommended Experimental Conditions and Parameters for $\Phi_{\Delta}$ Measurements via Steady-State Methods

\begin{tabular}{|c|c|c|}
\hline & recommended & possible limitations, remarks \\
\hline \multicolumn{3}{|c|}{ absolute method (chemical probe) } \\
\hline probe molecule & FFA & use purified FFA (i.e., vacuum-distilled) \\
\hline$k_{\mathrm{rxn}, 1 \mathrm{O} 2}^{\mathrm{FFA}}\left(\mathrm{L} \mathrm{mol}^{-1} \mathrm{~s}^{-1}\right)$ & $1.00 \times 10^{8}\left(22^{\circ} \mathrm{C}\right)$ & $\begin{array}{l}\text { correct for temperature dependence }\left(k_{\mathrm{rxn}, 1 \mathrm{OO} 2}^{\mathrm{FFA}}\left[\mathrm{L} \mathrm{mol}^{-1} \mathrm{~s}^{-1}\right]=1.00 \times 10^{8}+2.1 \times 10^{6}\left(\mathrm{~T}\left[{ }^{\circ} \mathrm{C}\right]-22\right) \text {, valid }\right. \\
\text { for distilled water }) \text { and ionic strength dependence }\left(k_{\mathrm{fxn}, 1 \mathrm{~F} 2}^{\mathrm{FFA}}\left[\mathrm{L} \mathrm{mol}^{-1} \mathrm{~s}^{-1}\right]=9.7 \times 10^{7}+1.2 \times 10^{7} \mathrm{C}_{\mathrm{NaCl}}\right. \\
\left.\left[\mathrm{mol} \mathrm{L}^{-1}\right] \text {, valid for } T=20{ }^{\circ} \mathrm{C} \text {; see Appiani et al. }{ }^{103}\right)\end{array}$ \\
\hline$k_{\mathrm{obs}, \mathrm{FFA}}\left(\mathrm{s}^{-1}\right)$ & $\begin{array}{l}{[\mathrm{FFA}]_{0}<145 \mu \mathrm{mol} \mathrm{L}{ }^{-1}} \\
\quad\left(5-40 \mu \mathrm{mol} \mathrm{L}^{-1}\right)\end{array}$ & $\begin{array}{l}\text { test for the importance of }{ }^{\bullet} \mathrm{OH} \text {; account for additional FFA reactivity using quenchers or by quantifying } \\
{\left[{ }^{\bullet} \mathrm{OH}\right]_{\mathrm{ss}} \text {; test for and correct for "apparent" direct photolysis (nondistilled FFA); follow kinetics for at }} \\
\text { least } 1 \text { half-life }\end{array}$ \\
\hline$k_{\mathrm{d}}^{\Delta}\left(\mathrm{s}^{-1}\right)$ & $2.76 \times 10^{5}\left(20^{\circ} \mathrm{C}\right)$ & small temperature dependence $\left(2.78 \times 10^{5}\right.$ at $25^{\circ} \mathrm{C}, 2.90 \times 10^{5}$ at $30^{\circ} \mathrm{C}$; see Appiani et al. $\left.{ }^{103}\right)$ \\
\hline irradiance method & actinometry & \\
\hline actinometer & PNA-py & $\begin{array}{l}\text { use recrystallized PNA; PNAP might be more appropriate for DOM with very low } \Phi_{\Delta} \text { (i.e., for long } \\
\text { reaction times) }\end{array}$ \\
\hline$\Phi_{\mathrm{dir}}^{\mathrm{PNA}}(-)$ & $0.29[\mathrm{py}]+2.9 \times 10^{-4}$ & value in water, valid from 300 to $400 \mathrm{~nm}$ (Laszakovitz et al. ${ }^{130}$ ) \\
\hline$\varepsilon_{\lambda}^{\mathrm{PNA}}\left(\mathrm{L} \mathrm{mol}^{-1} \mathrm{~cm}^{-1}\right)$ & Laszakovitz et al. ${ }^{130}$ & \\
\hline$k_{\mathrm{obs}, \mathrm{PNA}}\left(\mathrm{s}^{-1}\right)$ & & follow kinetics for at least 1 half-life \\
\hline$\alpha_{\lambda}\left(\mathrm{cm}^{-1}\right.$ or $\left.\mathrm{m}^{-1}\right)$ & $\alpha_{\lambda}=A_{\lambda} / l$ & $\begin{array}{l}\text { correct for baseline absorption and long-wavelength offsets; if } \alpha_{\lambda}<0 \text {, replace with } \alpha_{\lambda}=0 \text { (more } \\
\text { recommendations in section 3.2.1.4) }\end{array}$ \\
\hline \multicolumn{3}{|c|}{ reference method (chemical probe) } \\
\hline reference sensitizer & $\mathrm{PN}$ & cosolvent might be needed (i.e., acetonitrile) \\
\hline$\Phi_{\Delta}^{\mathrm{PN}}(-)$ & $1.01 \pm 0.03$ & value in water, valid from 337 to $436 \mathrm{~nm}$ (Schmidt et al. ${ }^{163}$ ) \\
\hline probe molecule & FFA & \\
\hline$k_{\mathrm{obs}, \mathrm{FFA}}^{\mathrm{DOM}}\left(\mathrm{s}^{-1}\right), k_{\mathrm{obs}, \mathrm{FFA}}^{\text {Sens,ref }}\left(\mathrm{s}^{-1}\right)$ & $\begin{array}{l}{[\mathrm{FFA}]_{0}<145 \mu \mathrm{mol} \mathrm{L}^{-1}} \\
\quad\left(5-40 \mu \mathrm{mol} \mathrm{L}^{-1}\right)\end{array}$ & $\begin{array}{l}\text { test for the importance of }{ }^{\bullet} \mathrm{OH} \text {; account for additional FFA reactivity using quenchers or by quantifying } \\
{\left[{ }^{\bullet} \mathrm{OH}\right]_{\mathrm{ss}} ; \text { follow kinetics for at least } 1 \text { half-life }}\end{array}$ \\
\hline$\alpha_{\lambda}^{\mathrm{DOM}}, \alpha_{\lambda}^{\mathrm{Sens, \textrm {ref }}}\left(\mathrm{cm}^{-1}\right.$ or $\left.\mathrm{m}^{-1}\right)$ & $\alpha_{\lambda}=A_{\lambda} / l$ & $\begin{array}{l}\text { correct for baseline absorption and long-wavelength offsets; if } \alpha_{\lambda}<0 \text {, replace with } \alpha_{\lambda}=0 \text {; (more } \\
\text { recommendations in section 3.2.1.4) }\end{array}$ \\
\hline
\end{tabular}

example, Partanen et al. ${ }^{39}$ used benzophenone (in acetonitrile) as the validation compound, and obtained $(38.9 \pm 2.0) \%$, which is in good agreement with the published value of $37 \%$. Absence of agreement with the literature hints to biases or errors in both the experimental design and/or the data analysis protocol and can be easily identified and corrected at the first stage of data collection. Similar to PN, benzophenone might not be the most appropriate validation compound for our purposes, mostly because of its low solubility in water. Again, there is a need for an optimal validation compound, which would have a well-characterized ${ }^{1} \mathrm{O}_{2}$ quantum yield between $0.5 \%$ and $5 \%$, would be soluble in water, and could be used in the UVB, UVA, and visible range. Some authors have used SRFA as their validation compound; ${ }^{42,48}$ however, the large spread in $\Phi_{\Delta}$ literature values (Table 3) makes it unsuitable for validation purposes.

In addition to a validation compound, we also propose to always measure at least one reference DOM sample (i.e., SRFA, SRNOM, or both; authors should also report the lot number of the isolate used) under the specific experimental conditions employed in each study. This value can later be used by other authors to (semi)qualitatively compare results across studies in a more reliable way (see section 4.2 and 4.3 for practical examples).

Our analyses also highlighted general principles and guidelines that should be considered for any future work based on chemical probe methods. In general, we recommend the use of FFA as ${ }^{1} \mathrm{O}_{2}$ probe under pseudo-first-order conditions, i.e., $[\mathrm{FFA}]_{0} \ll 145 \mu \mathrm{mol} \mathrm{L}{ }^{-1}$ (Table 4). This approach allows one to apply the steady-state approximation and to arrive to the apparent ${ }^{1} \mathrm{O}_{2}$ quantum yield via eqs 7 (absolute methods) or 14 (relative method). The observed FFA degradation rate constant $\left(k_{\mathrm{obs}, \mathrm{FFA}}\right)$ should be obtained from kinetic data (i.e., most authors obtained it from the slope of $\ln \left([\mathrm{P}] /[\mathrm{P}]_{0}\right)$ vs time plots $)$. It is also advisible to perform control experiments to verify that "apparent" direct degradation (especially if FFA was not freshly distilled), ${ }^{\bullet} \mathrm{OH}$-induced degradation, or any other degradation pathway do not introduce artifacts in $k_{\mathrm{obs}, \mathrm{FFA}}$. Note that ${ }^{\circ} \mathrm{OH}$-induced degradation is wavelength- and DOM-dependent, and also depends on the presence of other species, such as nitrate, iron, and halides. Furthermore, one should use updated literature parameters (summarized in Table 4) adjusted to their experimental conditions (i.e., temperature).

Our examination of the sources of error in determining $\Phi_{\Delta}$ values revealed that $R_{\mathrm{abs}}$ is the most likely source of error in all measurement approaches, implying that extra care should be used when measuring both absorption spectra and the absolute incident irradiance (in absolute methods). If $I_{0, \lambda}$ is measured with a spectroradiometer, one should include all possible setup-dependent correction factors to convert the radiometer output into in-solution incident irradiance (see Bolton and Linden ${ }^{127}$ ). However, this approach is not recommended as it might also be subject to operator errors that are impossible to spot and correct a posteriori. Instead, we encourage the use of the chemical actinometer PNA(P)-py as a way to quantify $I_{0, \lambda}$. In this case, it is advisible to obtain $\gamma$ via eq 25 , which relies only on the pseudo-first-order rate constant (and not initial rates, as eq 22) and does not necessarily require the use of recrystallized PNA.

To calculate $R_{\mathrm{abs}}$, we recommend the use of eq 18 (or one of its nonsimplified variants; see section S2). In the presence of additional chromophores or impurities, eq 19 should be used instead. In all cases, we advocate for the use of nonsimplified equations to quantify $R_{\mathrm{abs}}$. Another critical parameter, particularly when measuring solar-integrated quantum yields, is the upper integration wavelength $\left(\lambda_{\text {up }}\right)$, whose optimal value lies in general between 600 and $750 \mathrm{~nm}$. We recommend performing a preliminary data analysis to identify the optimal $\lambda_{\text {up }}$ for the particular DOM type analyzed. It is worth nothing 

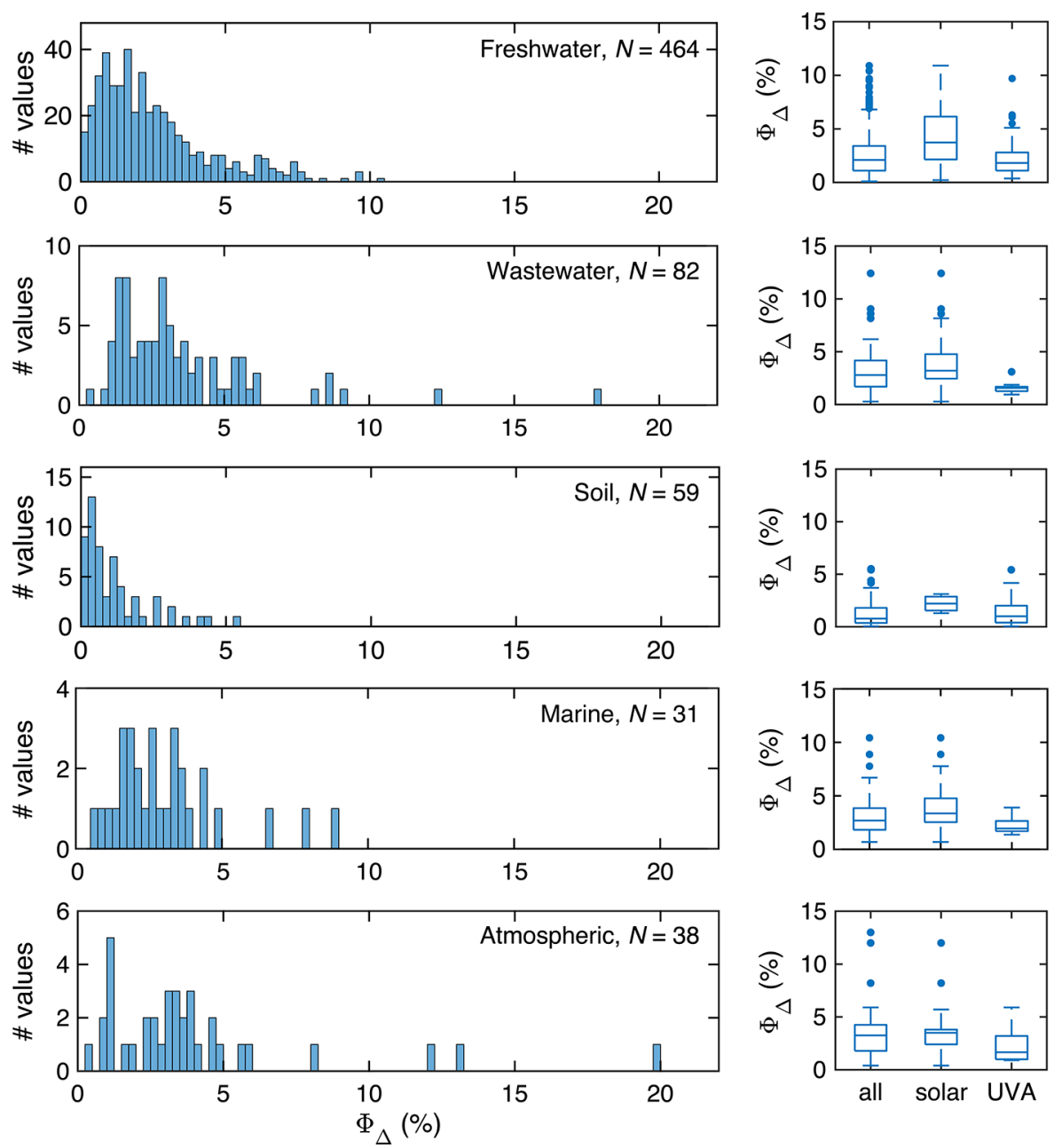

Figure 10. Overview of singlet oxygen quantum yields divided by environment (the database category "other DOM types" is not shown). Left: Data shown as histograms (bin size $=0.25 \%$ ). Right: Data shown as boxplots (all values, left; solar values, center; UVA values, right). In this analysis, we included only data for untreated and extracted DOM samples (database field "Treatment" = "none", "none (isolate *)", "extr").

that such an analysis can also offer insights on the presence of offsets in the absorption spectra (Figure 8B). In relative methods that use PN as the reference sensitizer, $\lambda_{\text {up }}$ is a less critical parameter, and can be generally be set to $650 \mathrm{~nm}$ (when using a broad band light source).

\section{SINGLET OXYGEN QUANTUM YIELDS IN ENVIRONMENTALLY RELEVANT SAMPLES}

In this section, we first present the structure of the ${ }^{1} \mathrm{O}_{2}$ quantum yield database that we compiled with the available data from the literature (section 4.1). Next, we discuss literature findings and general trends in apparent ${ }^{1} \mathrm{O}_{2}$ quantum yields as a function of DOM source environment (section 4.2, overview in Figure 10). In particular, we focus on freshwater DOM (section 4.2.1), wastewater DOM (section 4.2.2), soil organic matter (section 4.2.3), marine DOM (section 4.2.4), atmospheric organic matter (section 4.2.5), and other organic matter samples (section 4.2.6). We further provide an overview of isolation procedures for aquatic and soil samples (section 4.3.1) and atmospheric particulate matter (section 4.3.2), and we highlight possible biases on apparent ${ }^{1} \mathrm{O}_{2}$ quantum yield values. In section 4.4 , we briefly summarize the main finding of this section and we highlight main knowledge gaps to be address in future work.
The main purpose of section 4 is to provide a general overview of environments that have received the most attention to help identifying opportunities for future studies. When relevant, we also discuss general trends reported within selected studies. Semiquantitative comparisons across studies are performed only in terms of relative quantum yields, i.e., apparent ${ }^{1} \mathrm{O}_{2}$ quantum yields referred to the same reference material, typically one Suwannee River isolate. For consistency, these ratios are calculated among whole waters and reverse osmosis isolates (i.e., $\Phi_{\Delta}^{\text {whole }} / \Phi_{\Delta}^{\mathrm{SRNOM}}$ or $\Phi_{\Delta}^{\mathrm{NOM}} / \Phi_{\Delta}^{\mathrm{SRNOM}}$ ), fulvic acids (i.e., $\Phi_{\Delta}^{\mathrm{FA}} / \Phi_{\Delta}^{\mathrm{SRFA}}$ ), or humic acids (i.e., $\Phi_{\Delta}^{\mathrm{HA}} / \Phi_{\Delta}^{\mathrm{SRHA}}$ ) measured in the same study and under the same experimental conditions. If more than one value is available, relative quantum yields are reported as the average of the ratio calculated for each sample or for each study, and the error indicates the standard deviation of the ratios. Numerical data used in these calculations can be found in the ${ }^{1} \mathrm{O}_{2}$ database and were not subjected to harmonization or any type of correction as described in section 3.3. We want to stress that this way of reporting results does harmonize for variations in literature values, but might still be subjected to bias (as, for example, correction factors for the upper integration wavelength change based on DOM type; see section 3.3 and Figure S6A). For this reason, these results and discussions should be considered only semiquantitative. 
Table 5. Overview of Possible Values for Relevant Fields of the Apparent ${ }^{1} \mathrm{O}_{2}$ Quantum Yield Database

\begin{tabular}{|c|c|}
\hline field name & values \\
\hline treatment & none, none (isolate $*$ ), extr, photo, ox, red, extr-red, ads \\
\hline DOM origin & $\begin{array}{l}\text { freshwater (lake), freshwater (river), wastewater, soil, marine (estuarine), marine (coastal), marine (open ocean), marine (mariculture), } \\
\text { atmospheric (rain), atmospheric (fog), atmospheric (PM), atmospheric (lab SOA), other (algal), other (commercial), other (urban waste), } \\
\text { other (lab NOM), other (nanoparticle) }\end{array}$ \\
\hline reference $^{a}$ & $\begin{array}{l}\text { n, Ala FA, Ald HA, Contech FA, ESFA, ESHA, Fluka HA, Henan FA, LauFA, LauHA, LSHA, MRNOM, NLFA, NLHA, NLNOM, PLFA, PPFA, } \\
\text { PPHA, SRFA, SRHA, SRNOM, WPOM }\end{array}$ \\
\hline solvent ${ }^{b}$ & buffer (phosphate), buffer (acetate), buffer (borate), buffer (phosphate, D2O), water (DI), water (D2O), water (DI) + Fe + EDTA \\
\hline probe & FFA, 2,5-DMF, furoin, phosphorescence \\
\hline lamp & blue bulbs, laser, mercury, UVA bulbs, UVB bulbs, xenon, xenon (filters) \\
\hline light reference & $\begin{array}{l}\text { actinometry (2NBA), actinometry (ferrioxalate), actinometry (PNA-py), actinometry (PNAP-py), radiometry (bolometer), radiometry } \\
\text { (spectroradiometer), relative (PN), relative (RB), relative (SRFA), relative (TMPyP) }\end{array}$ \\
\hline
\end{tabular}

\subsection{Structure of the Database}

The database lists 857 apparent ${ }^{1} \mathrm{O}_{2}$ quantum yield values of environmental samples collected from 59 studies published between 1977 and March 2020. When available, these values were acquired from the main text or from tables within each publication. In most cases, apparent ${ }^{1} \mathrm{O}_{2}$ quantum yield values were presented in the main text in graphical forms, and their numerical values were listed in tables in the Supporting Information. When the numerical values were not present neither in the main text nor in the supplementary files, we contacted the corresponding authors (identified with an asterisk in the database field "Literature reference"). If we could not successfully get in contact with the authors, we extracted the most relevant quantum yield values from graphs using WebPlotDigitizer $4.2^{176}$ (identified with double asterisk in the database field "Literature reference" and colored in red). This strategy was applied only in a few cases, and only when it was possible to unambiguously identify the sample name from the available data. With an accurate calibration of the axis values, WebPlotDigitizer allowed us to extract data with good accuracy and precision (a linear regression analysis of measured vs extracted data $(N=40)$ provided slope $=$ $(1.0017 \pm 0.0013)$, intercept $=-(0.010 \pm 0.005)$, and $\mathrm{R}^{2}=$ $0.99993)$. In a limited number of cases, we could not contact the author, could not attribute an accurate value to the samples, or could not unequivocally identify the sample name from the plot legend. Therefore, we did not include these samples in the database ( $\sim 10-20$ values $)$.

For each entry, we first provide information on the original publication (fields "Literature reference" and "Publication DOI"), the publication year (field "Year"), and the sample name in the study (fields "Publication ID" and "Publication ID explained"). We further classify each sample based on its origin (field "DOM origin") and relevant treatments (field "Treatment"). The field "Treatment" identifies whole water samples ("none") from samples that were extracted ("extr"), subjected to general oxidative treatments ("ox"), photooxidized ("photo"), reduced with sodium borohydride ("red" or "extrred"), amended with halogens or other salts ("hal"), or adsorbed onto particles ("ads"). Note that samples classified as "none" include whole water samples that were used as received or filtered with $0.2 \mu \mathrm{m}, 0.45 \mu \mathrm{m}$, or $0.7 \mu \mathrm{m}$ filters. In most cases, such samples were stored at $4{ }^{\circ} \mathrm{C}$ or frozen for a limited period of time before analysis. Extracted samples ("extr") include samples subjected to solid phase extraction, size fractionation, humic and fulvic acid extraction, reverse osmosis, electrodialysis, and other isolation procedures. Note that commercially available isolates that were not subjected to additional treatment (besides dissolution and filtration) are identified as "none (isolate *)". We further report geographical coordinates expressed in decimal degrees (fields "Latitude (deg)" and "Longitude (deg)"); the field "Exact" indicates whether the coordinates are exact ("y", i.e., as indicated in the publication) or approximate ("n"). The field "Reference" tags commercially available samples including, among others, Suwannee River fulvic acid (SRFA), humic acid (SRHA), and natural organic matter (SRNOM), Pony Lake fulvic acid (PLFA), and Elliott soil humic acid (ESHA). A list of reference DOM samples, their commercial source, and their abbreviation is provided in Table S12. If available, we report total organic carbon concentrations (field "TOC"), solution $\mathrm{pH}$ (field "pH"), and solvent (field "solvent") of the apparent ${ }^{1} \mathrm{O}_{2}$ quantum yield experiment. TOC concentrations are normally in $\mathrm{mg}_{\mathrm{C}} \mathrm{L}^{-1}$; concentrations in $\mathrm{mg} \mathrm{L}^{-1}$ are shown in blue. Further, we report details pertinent to $\Phi_{\Delta}^{\mathrm{DOM}}$ measurements, namely the specific ${ }^{1} \mathrm{O}_{2}$ probe employed (field "Probe"), the lamp used (field "Lamp") and its approximate wavelength maximum (field "Wavelength"), the light reference used to obtain the absolute irradiance (field "Light reference"), and, when relevant, the integration range (field "Integration range"). Note that the wavelength maximum was rounded to either 5 or the closest decimal unit. When authors used a mercury lamp not equipped with a monochromator to select one of its emission lines, we indicate the emission range instead of the maximum intensity wavelength. Last, we report the apparent ${ }^{1} \mathrm{O}_{2}$ quantum yield value (field "1O2 QY value") and, if available, its error (field "1O2 QY error") and the E2:E3 value (field "E2E3"; authors that used as E2 the absorbance at 250 and $254 \mathrm{~nm}$ are indicated in blue and black, respectively). Table 5 summarizes the possible values of relevant fields present the database.

\subsection{DOM Types}

4.2.1. Freshwater DOM. Overall, freshwater environments have been the most studied in terms of $\Phi_{\Delta}^{\mathrm{DOM}}$, with 590 apparent ${ }^{1} \mathrm{O}_{2}$ quantum yield values available in the literature (out of $857,69 \%$ ). These values are available for widely diverse environments, including bogs, swamps, ponds, alpine lakes, Arctic and Antarctic lakes, great North American lakes, rivers, and streams, and span from negligible to $10.9 \%$ (for untreated samples; see Figure 10). So far, the majority of the studies focused on samples collected in temperate midlatitudes of North America and Europe, mostly in the geographical area between the Tropic of Cancer and the Arctic Circle. There are a few values available for Antarctic lakes (mostly relative to Pony Lake) and for Chinese rivers, while tropical and equatorial areas including South America, Africa, and East 

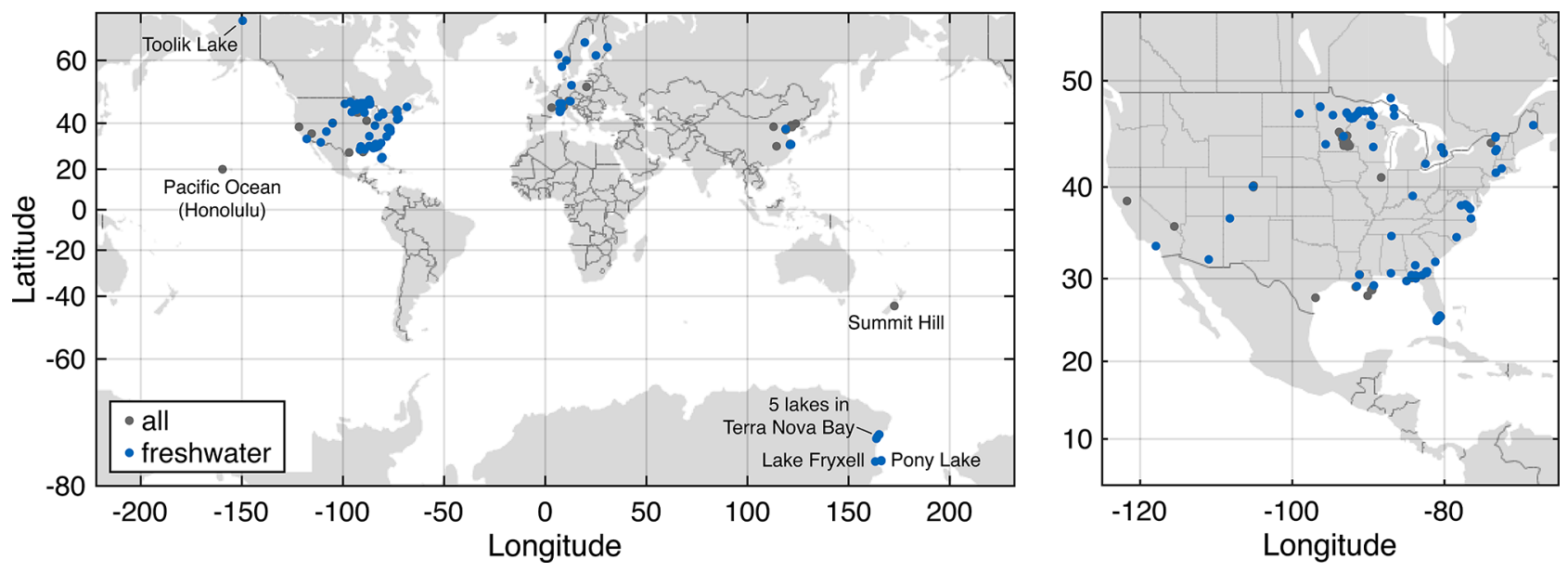

Figure 11. Geographical distribution of available apparent ${ }^{1} \mathrm{O}_{2}$ quantum yield values. The right panel is a zoomed view of North America. In this analysis, we included only data for untreated and extracted DOM samples (field "Treatment" = "none", "none (isolate *)", "extr"). The map is from MATLAB Basemap Data - grayterrain.

Asia are still largely unexplored (Figure 11). This focus is not surprising, and it is related to proximity of research laboratories and universities conducting the research. In addition, several authors have used freshwater DOM samples from the International Humic Substances Society (IHSS) only to provide a reference value, which partially inflates the true number of studies dealing with freshwater DOM. Among the most popular IHSS isolates for reference purposes are humic acids, fulvic acid, and natural organic matter from the Suwannee River, a blackwater river originating from Okefenokee Swamp in Georgia (US), ${ }^{177}$ and fulvic acids isolated from Pony Lake in Antarctica. A further reason for selecting Suwannee River and Pony Lake as references is their well-established difference in DOM source, with the former being a terrestrial (or allochthonous) end-member, and the latter a microbial (or autochthonous) DOM end-member. ${ }^{177-179}$ An overview of aggregated and corrected literature values for Suwannee River and Pony Lake isolates can be found in Table 3 and Table S11, respectively (bold values).

An inspection of the available literature supports general knowledge that PLFA (i.e., fulvic acid derived from microbially produced DOM) has a higher apparent ${ }^{1} \mathrm{O}_{2}$ quantum yield then SRFA (i.e., terrestrial fulvic acids), as $\Phi_{\Delta}^{\mathrm{PLFA}} / \Phi_{\Delta}^{\mathrm{SRFA}}=1.63$ $\pm 0.63(N=13){ }^{31,32,39,42,43,53-55,58,63,66,74,80,81}$ Nevertheless, we point out that a large scatter exists within experimental data, with $\Phi_{\Delta}^{\mathrm{PLFA}} / \Phi_{\Delta}^{\mathrm{SRFA}}$ values ranging from 0.72 to 3.07 (median of 1.58). In addition to Pony Lake, other freshwater environments that are instead characterized by terrestrial DOM inputs have higher photochemical reactivity compared to Suwannee River. For example, for Upper Mississippi River $\mathrm{NOM}, \Phi_{\Delta}^{\mathrm{MRNOM}} / \Phi_{\Delta}^{\mathrm{SRNOM}}=1.31 \pm 0.18(N=3),{ }^{32,39,53}$ while freshwater marshes from the Florida Everglades (US) with DOM inputs from soil and aquatic vegetation have $\Phi_{\Delta}^{\mathrm{NOM}} /$ $\Phi_{\Delta}^{\text {SRNOM }}=2.45 \pm 0.23(N=9) .{ }^{35}$ Organic matter from Scandinavian lakes have variable $\Phi_{\Delta}^{\text {NOM }} / \Phi_{\Delta}^{\text {SRNOM }}$ ratios from 0.70 to 3.96 (median of 1.30 ), with a mean value of $1.79 \pm$ $1.03(N=15){ }^{83}$ The photoreactivity of Nordic Lake, another boreal lake, seems to be more comparable to that of Suwannee River, even if differences exist between the fulvic acid fractions and the reverse osmosis isolates $\left(\Phi_{\Delta}^{\mathrm{NLNOM}} / \Phi_{\Delta}^{\mathrm{SRNOM}}=1.30 \pm\right.$ $0.41, N=6 ; \Phi_{\Delta}^{\mathrm{NLFA}} / \Phi_{\Delta}^{\mathrm{SRFA}}=0.72 \pm 0.12, N=$ 3). ${ }^{32,39,42,48,53,71,80,83}$ On the other hand, organic matter from the Great Dismal Swamp (Virginia, US), which receives input from decomposing plant material and is considered a terrestrial end-member, ${ }^{180}$ had $\Phi_{\Delta}^{\mathrm{NOM}} / \Phi_{\Delta}^{\mathrm{SRNOM}}=0.56 .{ }^{39}$ Other midlatitude swamps, bogs, and lakes have $\Phi_{\Delta}^{\mathrm{NOM}} / \Phi_{\Delta}^{\mathrm{SRNOM}}<1$ (Etang de la Gruère, 0.44; ${ }^{39}$ Lake Bradford, 0.78; ${ }^{39}$ Mt. Pleasant Lake, $0.83^{48}$ ). Thus, based on the available literature, microbial end members have higher apparent ${ }^{1} \mathrm{O}_{2}$ quantum yields compared to Suwannee River, while DOM with terrestrial contributions appear to have more variable $\Phi_{\Delta}$ values.

4.2.2. Wastewater DOM. Wastewater DOM (also called effluent organic matter, EfOM) is the second preferred DOM type in ${ }^{1} \mathrm{O}_{2}$ quantum yield studies, with 102 values (of 857 , $12 \%$ ) over 12 publications. A common motivation of these studies is to understand the effect of EfOM on the photochemistry of the receiving waters, ${ }^{40,41,55}$ as the discharge might impact the river's ability to degrade micropollutants via photochemical processes. From a molecular point of view, EfOM shares similarities with microbially derived natural organic matter, as it consists of a mixture of the influent NOM remaining after the wastewater treatment, soluble microbial products formed during the treatment process, as well as organic contaminants and their degradation products. ${ }^{41,55,63,78}$ Consistently, several authors have reported overall higher apparent ${ }^{1} \mathrm{O}_{2}$ quantum yields for EfOM compared to terrestrial DOM, ${ }^{40,78-80}$ similar to what has been observed for PLFA. In agreement with this view, $\Phi_{\Delta}^{\mathrm{EfOM}} / \Phi_{\Delta}^{\mathrm{SRNOM}}=1.85 \pm 0.34(\mathrm{~N}=$ $6) .{ }^{53,55,57}$ This result naturally leads to the idea that EfOMinfluencing receiving waters could have enhanced photoreactivity. However, this notion is tempered by the findings of Bodhipaksha and coauthors, ${ }^{40,41}$ who observed an absence of conservative mixing in ${ }^{1} \mathrm{O}_{2}$ quantum yields, and overall small variations in $\Phi_{\Delta}$ between samples collected upstream and downstream of wastewater treatment plans. Based on their calculations, the authors conclude that modest EfOM contributions $(<25 \%)$ are unlikely to impact the photochemistry (only ${ }^{1} \mathrm{O}_{2}$-related processes; other PPRIs behave differently) of receiving water bodies. ${ }^{41}$ The higher $\Phi_{\Delta}$ of EfOM compared to allochthonous DOM might be due to the high proportion of microbially derived material, the reduced molecular weight resulting from oxidative treatments (i.e., ozonation, chlorination, etc.), or a combination of these factors. ${ }^{55}$ Increased $\Phi_{\Delta}$ values as a function of oxidation is a 
well-established trend that will be discussed in more detail section 5.3.2.

4.2.3. Soil Organic Matter. Concerning soil organic matter (SOM), only 14 studies included samples from this environmental compartment, reporting a total of 62 values (of $857,7 \%$ ) ranging from $0.039 \%$ to $5.5 \%$ (Figure 10 ). Soil OM is typically subjected to alkaline extraction in order to isolate humic (HA; soluble at basic $\mathrm{pH}$ and insoluble at acidic $\mathrm{pH}$ ) and fulvic acids (FA; soluble at all $\mathrm{pH}$ ), which are later redissolved in water or buffer for photochemical experiments (see also section 4.3.1). We note that, despite being a wellestablished approach, the environmental significance of alkaline extracts is the subject of lively discussions in the soil chemistry community. ${ }^{181-183}$ Nkhili et al. ${ }^{49}$ also isolated the water-extractable (WE) fraction, the portion of SOM that can be obtained by simple agitation of the soil in neutral water, a procedure that is expected to better mimic leaching of organic matter from the soil to aquatic environments. For Elliott soil, a fertile prairie soil in Illinois, US, ${ }^{177}$ Nkhili and co-workers report ${ }^{1} \mathrm{O}_{2}$ quantum yields in the order of $\mathrm{FA}(5.4 \%)>\mathrm{HA}$ $(2.1 \%)>$ WE $(1.0-1.4 \%$, depending on the extraction temperature). ${ }^{49}$

Other authors used Elliott soil fulvic and humic acids, as they have been isolated by the IHSS and are commercially available. Other IHSS soil samples include Leonardite HA (naturally oxidized lignite), and Pahokee Peat humic and fulvic acids (agricultural soil in the Everglades, US). ${ }^{177}$ Non-IHSS commercial isolates such as Fluka HA and Sigma HA are also considered soil organic matter samples, and have been typically used for reference purposes (however, their use as reference is arguable given the well-known uncertainties on their origin and extraction protocols ${ }^{184}$ ). Only a handful of authors analyzed the ${ }^{1} \mathrm{O}_{2}$-sensitizing abilities of noncommercially available soilderived OM samples. For example, Aguer et al. ${ }^{69}$ reported higher $\Phi_{\Delta}$ for humic substances isolated from Ranker soil in eastern Europe (mountain acid soils; FA: 5.5\%; HA: 3.6\%) compared to Aldrich HA (2.0\%) and HA isolated from Rendzina, a forested soil on limestone (1.1\%). Also Frimmel and coauthors ${ }^{33}$ isolated fulvic acids from a Rendzina soil in Germany, while Nkhili et al. ${ }^{49}$ used carbon-rich fertile black soils from France and Poland to get water extractable-SOM. Analysis of the available literature data reveals that both fulvic and humic acids isolated from soil have overall higher photochemical reactivity than Suwannee River organic matter $\left(\Phi_{\Delta}^{\mathrm{HA}} / \Phi_{\Delta}^{\mathrm{SRHA}}=2.52 \pm 0.58, N=6 ; \Phi_{\Delta}^{\mathrm{FA}} / \Phi_{\Delta}^{\mathrm{SRFA}}=2.07 \pm 1.35\right.$, $N=3) .{ }^{32,33,83}$ However, we acknowledge that this trend might be biased toward differences in isolation procedures between aquatic and soil humic and fulvic acids (more details in section 4.3.1). Overall, there is still very limited knowledge on the variation of $\Phi_{\Delta}$ as a function of soil type and horizon, and on the effect of these variations on the photochemistry of the nearby water bodies.

4.2.4. Marine DOM. Similar to soil organic matter, only a limited number of peer-reviewed publications focused on marine DOM. Apparent ${ }^{1} \mathrm{O}_{2}$ quantum yield values are available for DOM isolates or whole water samples collected in estuarine (16 values), ${ }^{31,35,37,65}$ coastal (pristine and maricultureimpacted; 13 values), 3,31,35,37,53,64,65 and open-ocean environments ( 2 values). ${ }^{39,64}$ At the current state of knowledge, it is difficult to say how apparent ${ }^{1} \mathrm{O}_{2}$ quantum yields of marine DOM compare to freshwater samples. Sandvik et al. ${ }^{37}$ measured $\Phi_{\Delta}$ values of DOM isolates (obtained via ultrafiltration) along salinity transects of Mississippi and
Atchafalaya Rivers and observed overall constant quantum yield values $(1.4 \%-3.9 \%)$. On the other hand, Zhou et al. ${ }^{31}$ reported a clear decreasing trend from freshwater to seawater for whole water samples collected in the Bohai Bay, China (freshwater: $(7.67 \pm 0.25) \%$; estuarine water: $(6.70 \pm 0.35) \%$; seawater: $(1.94 \pm 0.04) \%)$, while Wang et al. $^{65}$ reported slightly higher values for coastal water isolates (via reverse osmosis) compared to terrestrially derived reference materials (coastal DOMs: $(3.36 \pm 0.46) \%$ and $(2.75 \pm 0.32) \%$; SRFA: $(2.20 \pm 0.10) \%)$. Other authors have also reported higher $\Phi_{\Delta}$ values for marine isolates compared to Suwannee River NOM. $^{39,53}$

Overall, marine DOM is characterized by a lower degree of aromaticity and lower chromophore and fluorophore content; ${ }^{185}$ it is therefore expected to exhibit photochemical behavior similar to that of autochthonous freshwater DOM (i.e., PLFA) and effluent organic matter. The fact that this trend is generally observed only in studies employing reverse osmosis isolates hints that both the isolation procedure and the presence of high halide concentrations might introduce biases in the final quantum yield values. Indeed, Sandvik et al. ${ }^{37}$ recognized that ultrafiltration might not be the most appropriate isolation procedure for marine samples, as it tends to remove low-molecular-weight material. Low-molecular-weight DOM is abundant in high salinity waters and it is typically characterized by high $\Phi_{\Delta}$. On the other hand, the effect of high halide concentrations on ${ }^{1} \mathrm{O}_{2}$ reactivity has not yet been thoroughly evaluated (see section 5.1) and could potentially impact FFA reactivity in high ionic strength solutions, ${ }^{103}$ affect DOM photophysics (as for triplet organic matter), ${ }^{186}$ or a combination of both. Furthermore, due to the limited number of samples, it is currently unknown if and how $\Phi_{\Delta}$ of marine samples change as a function of latitude and other geographical parameters.

4.2.5. Atmospheric Organic Matter. Concerning atmospheric samples, photosensitized singlet oxygen production has been investigated most extensively in hydrometeors. Hydrometeors include all forms of liquid and solid water in air, namely fog, cloud droplets, rain, and snow. These atmospheric samples are collected with simple equipment that does not introduce sampling bias, and therefore they can be considered atmospheric whole waters. For example, fog and cloud droplets can be collected with cloudwater collectors, ${ }^{6,8}$ which consist of water-repellant strings arranged as a sieve where water droplets can condense or adsorb on. ${ }^{187}$ Rainwater is sampled with simple buckets called bulk collectors, ${ }^{50}$ while snow can be picked up from the ground. ${ }^{11}$ So far, apparent singlet oxygen quantum yields have been measured only in fog $^{8}$ and rainwater, ${ }^{6,50}$ with values spanning the range of $(0.4 \pm 1.3) \%$ to $20 \%$ (14 values). Note that the first study of ${ }^{1} \mathrm{O}_{2}$ quantum yields in atmospheric samples has considerably higher values $(4.8-20 \%)^{6}$ than other reports $((0.4 \pm 1.3) \%-(8.2 \pm$ $1.5) \%),{ }^{8,50}$ suggesting that the actual range might be narrower.

Apparent ${ }^{1} \mathrm{O}_{2}$ quantum yields have also been measured in aerosols, both lab-generated secondary organic aerosols (SOAs) and authentic atmospheric samples. ${ }^{7,9}$ Aerosols samples were collected using PM filters $(2.5$ and $10 \mu \mathrm{m}$ for $\mathrm{PM}_{2.5}$ and $\mathrm{PM}_{10}$, respectively), and the water-soluble fraction was used for quantum yield measurements (more details on extraction procedures in section 4.3.2). For these extracts, $\Phi_{\Delta}$ values range from negligible to $(5.9 \pm 0.7) \%$ (24 values). ${ }^{7,9}$ Manfrin et al. ${ }^{9}$ also showed that synthetic SOAs exhibit different $\Phi_{\Delta}$ depending on the source material, with $\alpha$-pinene- 
derived SOAs having a considerably weaker ${ }^{1} \mathrm{O}_{2}$ photosensitizing ability compared to samples generated from aromatic precursors such as 1,8-dimethyl naphthalene. Thus, depending on the aerosol source material, $\Phi_{\Delta}$ values might display more significant variabilities than observed in terrestrial aquatic and oceanic samples.

4.2.6. Other Organic Matter Samples. Apparent singlet oxygen quantum yields have also been measured for other environmental samples that do not belong to the groups described above, such as dissolved black carbon (DBC), ${ }^{32,59}$ extracellular organic matter (EOM), ${ }^{62}$ urban waste-derived organic matter, ${ }^{61}$ synthetic organic matter samples, ${ }^{69,83}$ and humic acid-coated nanoparticles. ${ }^{84}$

Dissolved black carbon is the water-soluble fraction of black carbon, the residue formed during the incomplete combustion of biomass (biochar) and fossil fuels, and it is a ubiquitous component of DOM $(<2 \%$ to $19 \%$, depending on the environment). ${ }^{32,188,189}$ DBC is one of the least studied components of dissolved organic matter, even if previous work showed that it might be one of the most photoreactive ones. ${ }^{189,190}$ In agreement with this view, Du et al. ${ }^{32}$ measured considerably higher $\Phi_{\Delta}$ values for DBC obtained from combustion of $\mathrm{C} 3$ and $\mathrm{C} 4$ plants compared to standard humic acids (range DBC: $3.46-6.13 \%$, average DBC: $(4.52 \pm$ $0.98) \%, N=9$; range HA: $1.26-3.57 \%$, average HA: $(2.51 \pm$ $0.91) \%, N=9)$. Fu and coauthors ${ }^{59}$ obtained comparable results with biochar from bamboo shavings.

The term extracellular OM refers to organic material exuded by algae and mostly consists of polysaccharides, proteins, nucleic acids and lipids. ${ }^{62,191,192}$ Tenorio and co-workers ${ }^{62}$ investigated the photosensitizing abilities of EOM excreted by Chlamydomonas reinhardtii during the various phases of growth and observed $\Phi_{\Delta}$ values overall comparable to SRNOM, which was the reference material (EOM: 1.3-2.7\%; SRNOM: 1.4$2.5 \%)$. We also note that this study was conducted in the context of microalgae bioreactors for wastewater treatment purposes, meaning that these results might not be directly transferrable to EOM present in natural systems due to possible interference from the algae growing media, which contains EDTA, $\mathrm{Fe}^{3+}$, and bicarbonate. In a similar context, organic matter derived from home gardening and urban biowaste was investigated as a possible photosensitizer for wastewaters remediation. ${ }^{61}$ Preliminary results indicated that its apparent ${ }^{1} \mathrm{O}_{2}$ quantum yield is smaller or comparable to natural DOM (waste-derived OM: 1.4-2.3\%; SRNOM: $2.4 \%){ }^{61}$ suggesting that amendments of biowaste-derived OM might not improve the ${ }^{1} \mathrm{O}_{2}$-induced attenuation of micropollutants and pathogens already exerted by effluent organic matter, which is naturally present in the wastewater treatment plan.

A few authors measured apparent ${ }^{1} \mathrm{O}_{2}$ quantum yields of synthetic organic matter samples. In the context of atmospheric studies, Manfrin and coauthors ${ }^{9}$ generated "synthetic" secondary aerosols in a smog chamber in the presence of a SOA precursor, $\mathrm{H}_{2} \mathrm{O}_{2}$, and UVB light. Besides its limitations, ${ }^{193}$ this procedure is well-established in the atmospheric chemistry field and resulted in the production of aerosols whose $\Phi_{\Delta}$ values were comparable to authentic environmental samples (see section 4.2.5). ${ }^{9}$ Synthetic samples have also been produced in an attempt to mimic soil organic matter, but their environmental relevance is debatable. For example, Paul et al. ${ }^{83}$ used a synthetic sample produced via autoxidation of hydroquinone, which showed negligible ${ }^{1} \mathrm{O}_{2}$ production. Aguer et al. ${ }^{69,194}$ obtained comparable results using synthetic DOM generated via photooxidation (UVC) of a concentrated phenol solution.

Carlos and coauthors ${ }^{84}$ investigated the singlet oxygen sensitizing ability of soil-derived humic acids adsorbed on the surface of magnetite nanoparticles and found that humic acids are better ${ }^{1} \mathrm{O}_{2}$ sensitizers when freely dissolved in solution. For instance, Leonardite humic acid had $\Phi_{\Delta}=(0.40 \pm 0.02) \%$ and $(0.066 \pm 0.008) \%$ in the free and adsorbed form, respectively. Even if they did not report apparent quantum yields, Appiani and $\mathrm{McNeill}{ }^{195}$ reached the same conclusion when investigating the ${ }^{1} \mathrm{O}_{2}$ production ability of silica nanoparticles coated with Aldrich HA, which were used as surrogates for particulate organic matter (POM). Possible explanations for the lower $\Phi_{\Delta}$ of sorbed vs dissolved humic acids include increasing quenching of triplet excited states ${ }^{84,195}$ and selective uptake of poor ${ }^{1} \mathrm{O}_{2}$ sensitizers (i.e., hydrophobic DOM components) during particle coating. ${ }^{195}$ Despite the modest apparent quantum yields, Appiani and McNeill pointed out that particulate organic matter might still be an important photosensitizer for molecules adsorbed on POM due to the high ${ }^{1} \mathrm{O}_{2}$ levels on the particles surface. ${ }^{195,196}$

\subsection{Isolation and Extraction Procedures}

4.3.1. Aquatic and Soil Samples. Apparent ${ }^{1} \mathrm{O}_{2}$ quantum yields have been measured on both whole water samples and organic matter isolates. Standard isolation techniques in $\Phi_{\Delta}$ studies of aquatic and soil samples are solid-phase extraction (SPE), reverse osmosis (RO), reverse osmosis used in combination with electrodialysis, and (tangential flow) ultrafiltration. Lyophilization, sonication, and freeze-drying have also been used by some authors. Most of these techniques are suitable both to concentrate DOM, to isolate specific fractions based on hydrophobicity or operational definitions (i.e., humic and fulvic acids), and to remove ionic components of the environmental matrix that might interfere with ${ }^{1} \mathrm{O}_{2}$ quantum yield determinations.

Extraction of humic and fulvic acid is the most common isolation procedure for humic substances. For aquatic substances, these fractions are typically isolated via solidphase extraction using Amberlite XAD resins (Figure 12A), while, for soil samples, alkaline extraction is still the most popular technique (Figure 12B), despite criticisms on the environmental relevance of this approach. ${ }^{181-183}$ For soilderived fulvic acids, the IHSS used a hybrid procedure involving alkaline extraction followed by XAD-8 solid-phase extraction (Figure 12B). ${ }^{199}$ We also note that, despite the same name, aquatic and soil humic acids are operationally different, as the former are typically isolated from the hydrophobic acid fraction (HPOA), while soil HA obtained via alkaline extraction still contains both hydrophobic and hydrophilic fractions. In addition, even if well established, the XADisolation procedure selectively targets the hydrophobic acid fraction (Figure 12), meaning that apparent ${ }^{1} \mathrm{O}_{2}$ quantum yields measured for aquatic fulvic and humic acids and soil fulvic acids from the IHSS will not necessarily represent the value of the original natural sample.

So far, only three authors investigated the singlet oxygen sensitizing abilities of other DOM fractions. Partanen and coauthors $^{39}$ reported higher $\Phi_{\Delta}$ for hydrophobic organic neutral fractions (HPON) isolated from the Everglades and William lake as compared to hydrophobic acid and transphilic acids (TPIA) isolates. The authors hypothesized that this 

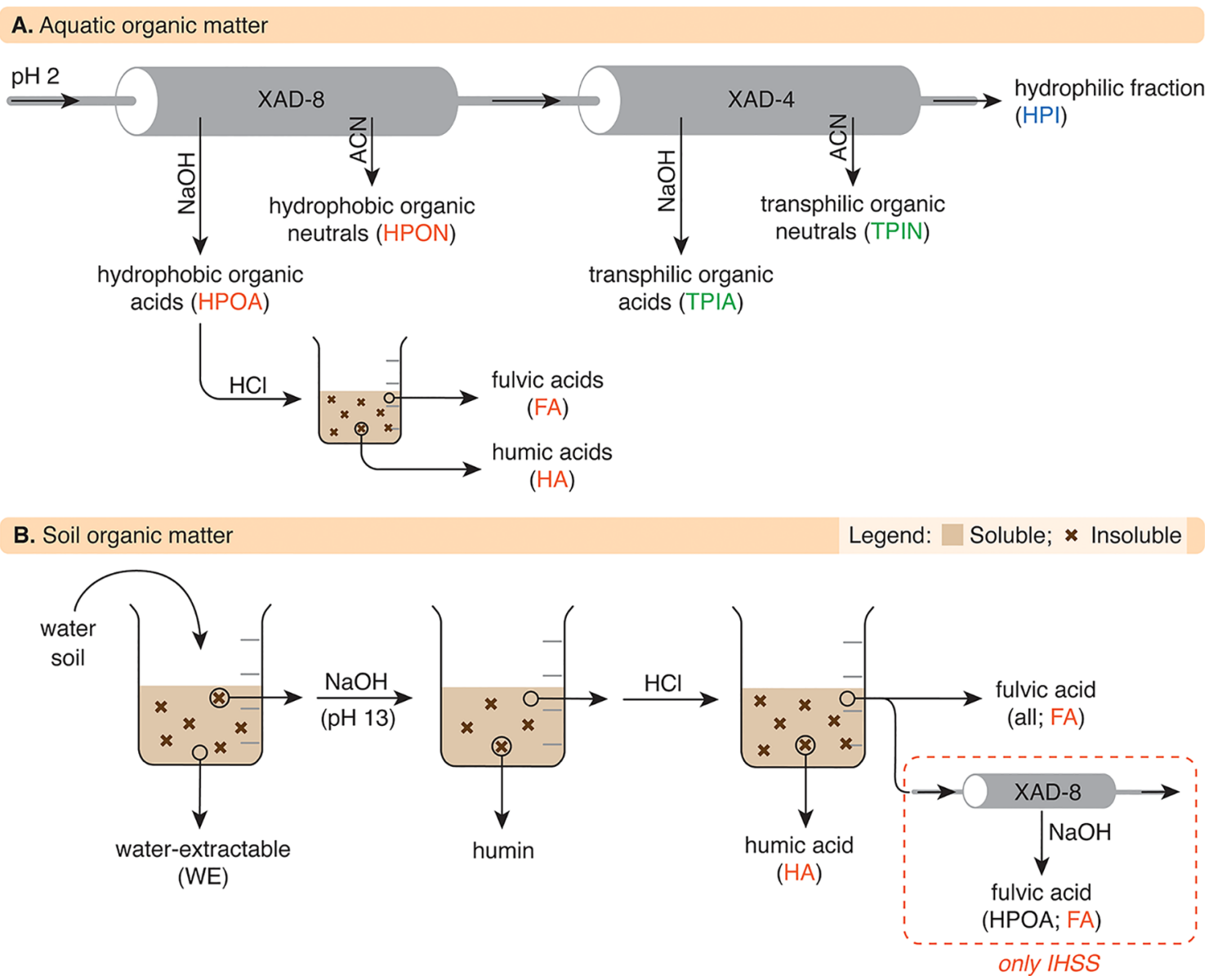

Figure 12. (A) Schematic procedure for extraction of aquatic DOM samples based on established XAD extraction protocols. ${ }^{197-199}$ Note that soil $\mathrm{HA}$ isolated via alkaline extraction contains both hydrophobic and transphilic fractions, and is not operationally equivalent to aquatic HA. ${ }^{199}$ Zhang et al. ${ }^{58}$ and Zhou et al. ${ }^{63}$ isolated a single hydrophobic (HPO) and transphilic (TPI) fraction eluting the resins adsorbates with methanol; thus, in these papers, HPO $\approx$ HPOA + HPON, while TPI $\approx$ TPIA + TPIN. (B) Schematic procedure for extraction of soil organic matter. ${ }^{49,182,199}$ Note that IHSS fulvic acid extracts contain only the HPOA fraction, ${ }^{199}$ while the regular alkaline extraction yields fulvic acids that contain all hydrophobicity fractions.

result might explain the lower ${ }^{1} \mathrm{O}_{2}$ quantum yield that they obtained for SRFA (1.4\%) and SRHA (0.6\%) compared to whole water samples (1.6\%) and reverse osmosis (1.8\%) Suwannee River isolates. This same picture is overall supported by analysis of other literature values for Suwannee River $\left(\Phi_{\Delta}^{\mathrm{SRNOM}} / \Phi_{\Delta}^{\mathrm{SRFA}}=0.92 \pm 0.30, N=9\right)^{32,52,54,55,63,65,71,80,83}$ and Nordic Lake $\left(\Phi_{\Delta}^{\mathrm{NLNOM}} / \Phi_{\Delta}^{\mathrm{NLFA}}=2.02 \pm 1.14, N=2\right) .^{32,48}$ Furthermore, two studies observed considerably higher $\Phi_{\Delta}$ values for hydrophilic fractions (HPI; 8.2-9.0\%) as compared to hydrophobic fractions $(3.2-4.0 \%)$, transphilic fractions (2.5-5.3\%), and whole waters $(2.7-6.1 \%)$ for effluent organic matter samples collected in wastewater treatment plants in China. 58,63 The authors speculated that HPI might be the largest source of ${ }^{1} \mathrm{O}_{2}$ due to the high percentage of proteins and peptides, and potentially because of lower ${ }^{1} \mathrm{O}_{2}$ quenching rate constants as compared to the hydrophobic and transphilic fractions. ${ }^{58}$ Besides these sparse reports, little is currently known on the effect of hydrophobicity fractionation on apparent ${ }^{1} \mathrm{O}_{2}$ quantum yields.

In addition, analysis of literature values indicates that both aquatic and soil humic acids have overall lower apparent ${ }^{1} \mathrm{O}_{2}$ quantum yields than fulvic acids isolated from the same source. Indeed, for Suwannee River, $\Phi_{\Delta}^{\mathrm{HA}} / \Phi_{\Delta}^{\mathrm{FA}}=0.63 \pm 0.13(N=$ $10),^{31,39,46,53-55,58,63,80,83}$ for Nordic Lake, $\Phi_{\Delta}^{\mathrm{HA}} / \Phi_{\Delta}^{\mathrm{FA}}=0.58(N$
$=1),{ }^{48}$ for Elliot soil, $\Phi_{\Delta}^{\mathrm{HA}} / \Phi_{\Delta}^{\mathrm{FA}}=0.33 \pm 0.08(N=2),{ }^{49,83}$ while for Pehokee Peat $\Phi_{\Delta}^{\mathrm{HA}} / \Phi_{\Delta}^{\mathrm{FA}}=0.58(N=1){ }^{32}$

Besides XAD, other solid-phase extraction (SPE) resins have been used to isolate DOM from salts or other water-soluble components. In particular, several authors employed PPL cartridges (a styrene-divinylbenzene polymer) to isolate effluent organic matter from its matrix. ${ }^{40,66,75,80}$ Maizel and Remucal $^{75}$ quantitatively assessed the effects of SPE on optical and photochemical parameters of freshwater and wastewater DOM, observing no statistically significant differences in $\mathrm{SUVA}_{254}, \mathrm{E} 2: \mathrm{E} 3$, and rate of light absorption compared to standardized whole water solutions (i.e., adjusted to match $\mathrm{pH}$ and [DOC] of SPE isolate solutions). For quantum yields and quantum yields coefficients of several PPRIs, they observed only a slight increase compared to values measured in standardized whole water solutions. For example, for ${ }^{1} \mathrm{O}_{2}$, they report an average quantum yield increase of $(21 \pm$ $15) \%{ }^{75}$ In agreement with this finding, control experiments performed by others showed no or minimal increase of $\Phi_{\Delta}$ upon SPE extraction. $35,40,66$

Reverse osmosis (RO) is a high throughput technique that allows processing large volumes of water with minimal interferences. ${ }^{200}$ Different from XAD-8 resin isolation, isolates obtained via RO still contain hydrophobic and hydrophilic 
fractions, as well as other naturally occurring organic solutes, which makes them highly representative natural organic matter samples. ${ }^{199,200}$ The IHSS provides RO isolates, including Suwannee River NOM and other reference NOM samples that have been used by several authors. ${ }^{199}$ Besides these isolates, only the work by Paul et al. ${ }^{83}$ has a relatively large data set of apparent ${ }^{1} \mathrm{O}_{2}$ quantum yields for non-IHSS reverse osmosis isolates, which were obtained in the context of a larger project. Experimental results by Partanen and co-workers ${ }^{39}$ confirm the suitability of RO as an extraction technique, as evidenced that, under the same irradiation conditions, the $\Phi_{\Delta}$ of a Suwannee River whole water sample (collected close to the IHSS collection site) had a comparable ${ }^{1} \mathrm{O}_{2}$ quantum yield to the one measured for the IHSS isolate (whole water: $1.6 \%$; RO isolate: $1.8 \%$; error $\sim 10 \%$ ).

Reverse osmosis coupled to electrodialysis (RO/ED) is a popular technique for isolating marine DOM samples for apparent ${ }^{1} \mathrm{O}_{2}$ quantum yield studies. ${ }^{53,64,65}$ This technique was also employed to isolate the Mississippi River NOM IHSS 2013 sample. ${ }^{199}$ This system simultaneously allows to isolate NOM without losing representative organic matter fractions and decrease its salt content, and thus combines advantages of RO and SPE. On the negative side, typically salts are not entirely removed, as can be achieved with SPE. ${ }^{201}$ As we discussed in section 4.2.4, seawater matrix effects observed for marine samples make it hard to assess whether RO/ED introduces biases in $\Phi_{\Delta}$ measurements. Based on the results available for RO, we expect this technique to be similarly robust; however, further studies are needed to confirm the absence of artifacts.

Ultrafiltration or tangential flow ultrafiltration are other isolation techniques that have been employed both to isolate and to fractionate DOM (see also section 5.2.1). ${ }^{37,48,83}$ Ultrafiltration exploits the larger hydrodynamic radius of high molecular weight material compared to other dissolved inorganic species. ${ }^{22}$ Besides removing salts, this process inherently leads to selective isolation of high molecular weight DOM fractions, ${ }^{202}$ which are not necessarily representative of the original DOM sample. This fractionation might be a potential problem when comparing ${ }^{1} \mathrm{O}_{2}$ quantum yields of DOM samples collected in different environments, as they might be characterized by different size distributions. For example, Sandvik et al. ${ }^{37}$ showed that $\Phi_{\Delta}$ values of ultrafiltrate marine DOM samples are considerably lower than the true quantum yield, as ultrafiltration removes a significant portion of the low-molecular-weight material responsible for the high $\Phi_{\Delta}$ of autochthonous DOM samples. This result was also supported by the noticeable change in spectral slope between whole waters and the ultrafiltrates. ${ }^{37}$

Lyophilization is a convenient technique to concentrate DOM samples, but has only rarely been used in $\Phi_{\Delta}$ studies. $^{37,86}$ In these studies, DOM was lyophilized to prepare more concentrated solutions in a different solvent $\left(\mathrm{D}_{2} \mathrm{O}\right)$, which was needed to overcome the low sensitivity of the ${ }^{1} \mathrm{O}_{2}$ detection method (steady-state ${ }^{1} \mathrm{O}_{2}$ phosphorescence). ${ }^{37,86}$ Due to its limited application, the effect of lyophilization on apparent singlet oxygen quantum yields is currently unknown.

Authors working with dissolved black carbon adopted a stepwise extraction protocol involving the dissolution of solid black carbon samples in deionized water followed by sonication, filtration $(0.45 \mu \mathrm{m})$, and freeze-drying of the filtrate. $^{32,59,203}$ This procedure resembles the one employed to extract organic matter from PM filters (see section 4.3.2), except for the sonication step. Even if sonication is featured in standard extraction protocols (also for PM filter extracts; see for instance Roper et al. ${ }^{204}$ ), Milijevic and coauthors ${ }^{205}$ have warned that reactive oxygen species can also be formed during short sonication periods (i.e., $15 \mathrm{~min}$ ). These reactive species react with organic matter, artifactually modifying the chemical composition of the extract. Future research needs to establish if sonication-induced changes have an effect on apparent ${ }^{1} \mathrm{O}_{2}$ quantum yield values.

4.3.2. Atmospheric Samples. In the context of atmospheric chemistry, the term organic matter isolate generally indicates particulate material isolated from the air, either the natural atmosphere or a smoke chamber. In a typical procedure, the particulate material $\left(\mathrm{PM}_{2.5}\right.$ or $\mathrm{PM}_{10}$, depending on the filter pore size) is first collected by passing air through filters with the help of a vacuum pump. The filters are later submerged in deionized water at room temperature for several hours to extract the water-soluble PM fraction. ${ }^{7,9,10}$ The extract can be further filtered (i.e., with $0.22 \mu \mathrm{m}$ filters) ${ }^{7}$ to remove insoluble particles and aggregates that might cause light scattering during the photochemical experiment. ${ }^{7,10}$ However, experiments performed on road dust suggested that the particles removed during this additional filtration step are also good ${ }^{1} \mathrm{O}_{2}$ sensitizers, ${ }^{10}$ similarly to what is known for aquatic particular organic matter. ${ }^{195}$ Thus, atmospheric extracts might show only a fraction of the photochemical reactivity present in the natural environment, similar to what discussed for aquatic extracts (section 4.3.1). Note also that this extraction protocol is the most common in ${ }^{1} \mathrm{O}_{2}$ photochemistry studies, but it is not the only available one. ${ }^{204,206}$

Furthermore, the use of water as extraction solvent targets only the water-soluble sensitizers present within the particulate matter. Work by Cote et al. ${ }^{10}$ showed that hexane-soluble chromophores are not a significant portion of the pool of ${ }^{1} \mathrm{O}_{2}$ sensitizers in road dust samples. However, more polar organic solvents such as methanol or acetonitrile, or multisolvent extraction techniques, ${ }^{207}$ might help extract additional pools of ${ }^{1} \mathrm{O}_{2}$ sensitizers from PM samples, allowing the assessment of the true ${ }^{1} \mathrm{O}_{2}$ photosensitizing ability of atmospheric organic matter. Alternatively, one could use a liquid impinger, which is often used to sample bioaerosols, ${ }^{208,209}$ to directly collect whole aerosol samples in water.

\subsection{Short Summary and Research Gaps}

So far, freshwater environments in the northern boreal latitudes have received the most attention in terms of apparent ${ }^{1} \mathrm{O}_{2}$ quantum yield measurements. Nevertheless, the lack of standardization and shared protocols makes it hard to include all of the available data in a global framework, and to use it to advance our understanding of DOM photochemistry. For example, we still lack convincing evidence that microbial DOM has higher $\Phi_{\Delta}$ than terrestrial DOM, even if this trend is assumed valid by most researchers in our field. For this reason, we advocate for the development of a shared and approved protocol for apparent quantum yield measurements (see also section 3.4). Such a protocol needs to include a validation step and the measurement of one of two reference DOM samples (i.e., SRFA, SRNOM, or both). The reference samples are needed to "normalize" quantum yield values for the particular experimental conditions and, eventually, perform meaningful semiqualitative comparisons across studies. 
Besides boreal freshwaters, many environments have received little attention so far in terms of photochemical reactivity studies. Specifically, tropical and equatorial regions appear highly understudied, and to date, there are no data for South America, Africa, Australia, and Middle East Asia. Soil organic matter has also received little attention, likely because most of the studies focused on processes occurring in aqueous phases, not on soil surfaces. Less understandably, only a few studies investigated the ${ }^{1} \mathrm{O}_{2}$ sensitizing ability of marine DOM. Besides basic knowledge, understanding DOM photoreactivity in coastal environments might be beneficial for modeling micropollutants degradation in areas exploited for mariculture or other anthropogenic activities. In the past years, there has also been an increased interest in measuring $\Phi_{\Delta}$ of various atmospheric samples and dissolved black carbon, which is considered one of the most photoactive components of DOM. For these DOM types, many open questions remain. Besides DOM, investigations on the role of particulate organic matter in producing ${ }^{1} \mathrm{O}_{2}$ and affecting the production of singlet oxygen and other PPRIs from DOM is still in its infancy. Given the ubiquitous presence of suspended particles in aquatic environments, we anticipate this to be a relevant area of future research. To this end, researchers should take inspiration from other fields (for instance, see Bregnhøj et al. ${ }^{210}$ and references therein) to develop a robust protocol to correctly and accurately quantify DOM's and POM's rate of light absorption in the presence of suspended particles.

In all of these environments, the selection of isolation or extraction technique is a critical point of apparent quantum yield measurements, as different procedures isolate different DOM pools that are not expected to show the same photoreactivity. In our opinion, the selection of the isolation or extraction procedure should be motivated by the general aim of the study. Whole water samples or reverse osmosis isolates are more appropriate for modeling studies, as they provide a more complete picture of the photoreactivity in environmentally relevant conditions. Solid-phase extraction can be a convenient choice when one wants to or needs to reduce salt content; however, one must be aware that both $\mathrm{XAD}$ resins and other SPE techniques selectively retain specific DOM components. ${ }^{197,198,211-213}$ The choice of whether or not to include the environmental matrix is particularly critical in marine DOM studies, as salt-free samples have a different photoreactivity than whole waters. Thus, we argue that modeling studies should include the environmental matrix, while authors interested in understanding photoreactivity differences between organic matter samples from different environments might prefer to use marine DOM isolates. On the other hand, lyophilization or/and rotary evaporation ${ }^{198}$ might be a convenient yet underexplored choice to concentrate diluted freshwater samples. Furthermore, the use of apparent ${ }^{1} \mathrm{O}_{2}$ quantum yields of aquatic humic and fulvic acids might not be appropriate for modeling purposes. Preliminary results from a few research groups have hinted that fulvic and humic acids, which are part of the hydrophobic organic acid fraction, have overall lower $\Phi_{\Delta}$ than the remaining transphilic and hydrophilic fractions and of whole water samples. Between the two fractions, humic acids appear to have consistently lower $\Phi_{\Delta}$ than fulvic acid isolated from the same organic matter sample; the reason for this difference is still an active area of research. Furthermore, care must be used when comparing apparent ${ }^{1} \mathrm{O}_{2}$ quantum yields of soil and aquatic humic acids, as, despite the name, they are operationally different. ${ }^{199}$ Similarly, atmos- pheric PM extracts have mostly characterized the photoreactivity of water-soluble sensitizers, which might not represent the entirety of the ${ }^{1} \mathrm{O}_{2}$ sensitizing pool. Further work should focus on improving the extraction efficiency (i.e., using different solvents or multisolvent extraction methods) to be able to better and more fully characterize the photoreactivity of atmospheric samples.

\section{TRENDS AND INTERPRETATION OF RESULTS}

In the following sections, we describe literature findings related to $\Phi_{\Delta}$ values of environmental samples, including trends as a function of bulk water chemistry parameters (section 5.1); spectroscopic, photochemical, and molecular DOM features (section 5.2 and Table 6), and chemical modifications of DOM (section 5.3). We further describe known wavelength trends (section 5.4), as well as spatial and temporal variations in apparent ${ }^{1} \mathrm{O}_{2}$ quantum yields (section 5.5). Due to the challenges of comparing values obtained by different authors, we focus on qualitative and, when possible, semiquantitative views of the available literature. In section 5.6, we briefly summarize the main finding of this section and we highlight main knowledge gaps to be address in future work.

\subsection{Water Chemistry}

Several authors have observed a negative correlation between $\Phi_{\Delta}$ and $\mathrm{pH}$, i.e., high $\Phi_{\Delta}$ at low $\mathrm{pH} .{ }^{33,43,48,75,82}$ This trend correlates to changes in DOM absorbance as a function of $\mathrm{pH}$, which results in increasing $R_{\mathrm{abs}}$ from acidic to basic $\mathrm{pH}^{75}$ Sharpless and Blough interpreted the variation in $\Phi_{\Delta}$ and spectroscopic properties as a function of $\mathrm{pH}$ in terms of a charge transfer (CT) model. ${ }^{214}$ They suggested that deprotonation of aromatic acids at basic $\mathrm{pH}$ increases the likelihood of forming charge transfer complexes, which in turn causes a red-shift in the absorption spectra (thus, an increase in $R_{\mathrm{abs}}$ ) and a decrease in apparent ${ }^{3} \mathrm{CDOM}^{*}$ quantum yield. On the other hand, the effect of $\mathrm{pH}$ on the reactivity of the chemical probe is minimal or absent, ${ }^{75,215}$ in agreement with the fact that both FFA and 2,5-DMF do not have ionizable groups at environmentally relevant $\mathrm{pH}$ values. ${ }^{103}$ In early work, Zepp and coauthors ${ }^{215}$ warn that, above $\mathrm{pH} 10,{ }^{1} \mathrm{O}_{2}$ quenching by $\mathrm{OH}^{-}$should be taken into account in $\Phi_{\Delta}$ calculations.

One recent study reported a strong positive trend of $\Phi_{\Delta}$ as a function of $\mathrm{pH}^{85}$ but this result has not been replicated by others. A possible explanation is that apparent ${ }^{1} \mathrm{O}_{2}$ quantum yields were obtained using visible light at $532 \mathrm{~nm}$; thus, at these high wavelengths, $R_{\mathrm{abs}}$ might display an opposite trend as a function of $\mathrm{pH}$. However, literature precedent allows us to rule out this hypothesis (see, for instance, Schendorf et al. ${ }^{216}$ ). Furthermore, $\Phi_{\Delta}$ in the visible range is typically 10 -fold lower than in the UVA and UVB region, which might increase the relative error associated with the quantum yield determination. Additionally, Leresche et al. ${ }^{43}$ observed a positive correlation between $\Phi_{\Delta}$ and $\mathrm{pH}$ for SRFA and PLFA samples treated with high $\mathrm{O}_{3}$ doses, which they interpreted qualitatively as due to different products distribution as a function of ozonation $\mathrm{pH}$.

Dissolved oxygen is another bulk water parameter that has been investigated. In particular, Sharpless ${ }^{71}$ observed a decrease of apparent ${ }^{1} \mathrm{O}_{2}$ quantum yields (at $370 \mathrm{~nm}$ ) below air saturation $\left(\left[\mathrm{O}_{2}\right]=0-0.29 \mathrm{mmol} \mathrm{L} \mathrm{L}^{-1}\right)$ and no changes above air saturation $\left(\left[\mathrm{O}_{2}\right]=0.29-1.4 \mathrm{mmol} \mathrm{L}^{-1}\right)$. Other authors reported constant $\Phi_{\Delta}$ or rate constants of the ${ }^{1} \mathrm{O}_{2}$ probe as a function of $\left[\mathrm{O}_{2}\right]$ above air saturation. ${ }^{68,70}$ This trend qualitatively agrees with our understanding of ${ }^{1} \mathrm{O}_{2}$ production 
in the natural environment, as $\Phi_{\Delta}$ is expected to change as a function of dissolved oxygen according to eq 2 . The relationship between $\Phi_{\Delta}$ and $\left[\mathrm{O}_{2}\right]$ has two significant consequences. First, $\Phi_{\Delta}$ is expected to change considerably as a function of $\mathrm{O}_{2}$ in environmentally relevant ranges (Figure 13A). Even though near-surface waters are typically oxic, their
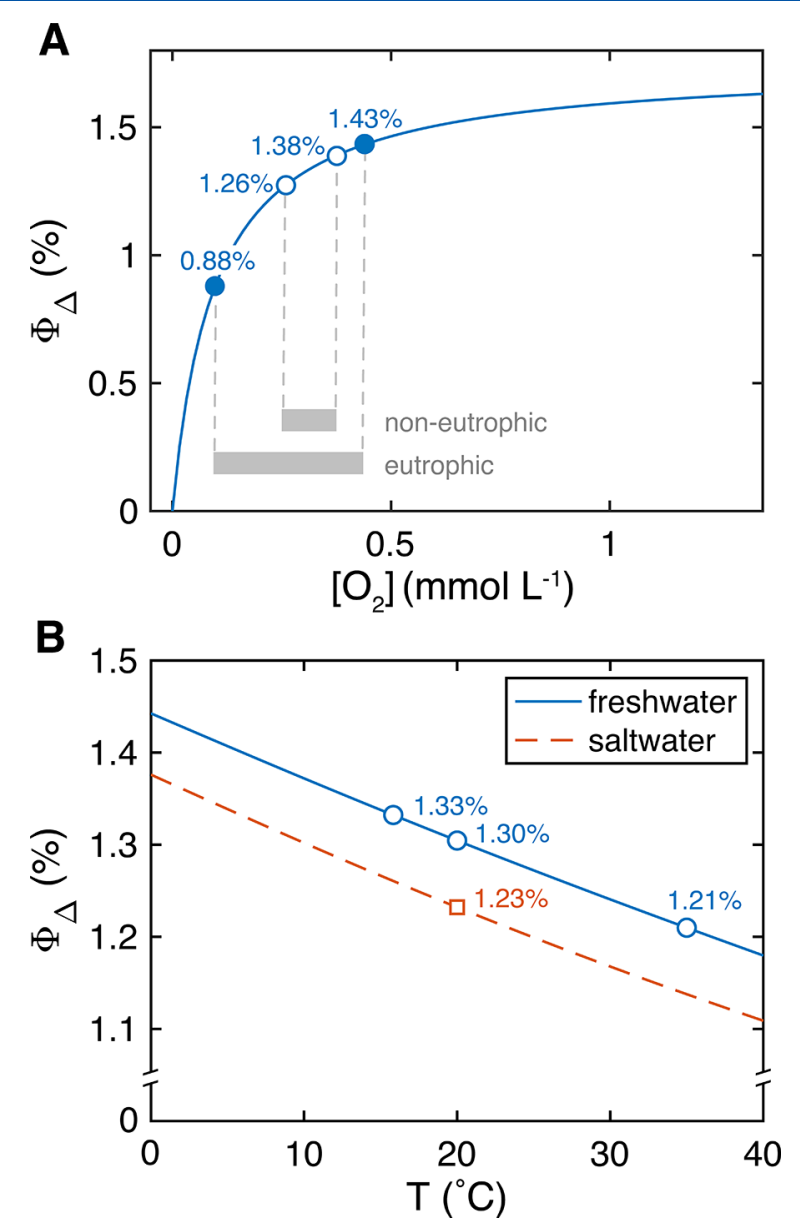

Figure 13. (A) Changes in $\Phi_{\Delta}$ as a function of dissolved $\mathrm{O}_{2}$ (eq 2) computed for SRNOM using literature data $\left(\Phi_{\mathrm{T}}=4.1 \%,{ }^{95} k_{\mathrm{O} 2}=8.1\right.$ $\times 10^{8} \mathrm{~L} \mathrm{~mol}^{-1} \mathrm{~s}^{-1}, 222 k_{\mathrm{d}}^{\mathrm{T}}=7.8 \times 10^{4} \mathrm{~s}^{-1}, 222 f_{\Delta}=0.426 ; f_{\Delta}$ was obtained from the previous parameters using $\Phi_{\Delta}=1.30 \%$ at $\left[\mathrm{O}_{2}\right]=$ $254 \mu \mathrm{mol} \mathrm{L}{ }^{-1}$ from Partanen et al. ${ }^{39}$ ). Highlighted data indicate computed diurnal changes in $\Phi_{\Delta}$ in eutrophic (filled symbols) and noneutrophic (empty symbols) streams with oxygen saturation at $\sim 10$ $\mathrm{mg} \mathrm{L}^{-1}\left(0.31 \mathrm{mmol} \mathrm{L}^{-1}, 15^{\circ} \mathrm{C}\right) .^{96}$ (B) Changes in $\Phi_{\Delta}$ as a function of temperature (at oxygen saturation) for freshwater and saltwater. Oxygen concentration at saturation in this temperature interval ranges from 6.4 to $14.6 \mathrm{mg} \mathrm{L}^{-1}\left(0.20-0.45 \mathrm{mmol} \mathrm{L}^{-1}\right) .{ }^{96}$

$\mathrm{O}_{2}$ concentration can vary as a function of biotic and abiotic processes occurring within the water body. ${ }^{96,217}$ For example, dissolved oxygen is known to have a diel cycle in streams and rivers, which is due to a balance of photosynthetic activity and heterotrophic consumption. ${ }^{96}$ This variation is considerably enhanced in eutrophic streams, which, also during daylight, can experience dissolved oxygen variations of half an order of magnitude (Figure 13A). ${ }^{96,218}$ In addition, water bodies with high organic matter content are typically undersaturated due to photochemical or biological consumption processes. ${ }^{96,217,219}$ Significant oxygen variations in aquatic environments are also expected to occur as a result of climate change. ${ }^{220}$ The second important aspect is that $\left[\mathrm{O}_{2}\right]$, thus $\Phi_{\Delta}$, change as a function of temperature, salinity, and pressure. ${ }^{96,221}$ We calculated that $\Phi_{\Delta}$ should decrease almost linearly as a function of temperature in the range $0-40{ }^{\circ} \mathrm{C}$ (Figure 13B). Even though this effect causes minimal changes in $\Phi_{\Delta}$ (estimated $\pm 7 \%$ variation for $\Delta T=15{ }^{\circ} \mathrm{C}$ ), it might explain part of the variability in literature values, as apparent ${ }^{1} \mathrm{O}_{2}$ quantum yields measurements have been conducted between 20 and $33{ }^{\circ} \mathrm{C}$ (Table S6). Similarly, increasing the salinity from 0 to $35 \mathrm{~g} \mathrm{~kg}^{-1}$ should decrease $\Phi_{\Delta}$ by $\sim 5 \%$ due to changes in dissolved $\mathrm{O}_{2}$ (Figure 13B).

So far, the effect of increasing salt content on $\Phi_{\Delta}$ has only been partially explored, one reason being the large preference on freshwater systems in apparent ${ }^{1} \mathrm{O}_{2}$ quantum yield studies. A few authors reported (weak) positive correlations between $\Phi_{\Delta}$ and salt content. For instance, McCabe and Arnold ${ }^{34}$ noted a significant correlation $\left(R^{2}=0.51\right)$ between $\Phi_{\Delta}$ and conductivity (range $0-5 \mathrm{mS} \mathrm{cm}^{-1}$ ) in whole water samples collected in temporary and semipermanent prairie pothole wetlands near Jamestown, North Dakota (US). On the other hand, McKay and coauthors ${ }^{35}$ found a weak positive trend $\left(R^{2}\right.$ $=0.17$ ) between $\Phi_{\Delta}$ and salinity (range 0-40 salinity units) in whole water samples collected in the Everglades, Florida (US). In these studies, variations in apparent ${ }^{1} \mathrm{O}_{2}$ quantum yields might be the result of several factors, including change in DOM type. Glover and Rosario-Ortiz ${ }^{54}$ performed a more systematic assessment of the effect of halides on apparent ${ }^{1} \mathrm{O}_{2}$ quantum yields using reference materials, and found a systematic enhancement in $\Phi_{\Delta}$ as a function of increasing chloride and bromide content. For example, for SRFA, they reported an increase in quantum yield from $0.37 \%$ (reference) to $3.16 \%$ and $3.19 \%$ for $\left[\mathrm{Cl}^{-}\right]=500 \mathrm{mmol} \mathrm{L}^{-1}$ and $\left[\mathrm{Br}^{-}\right]=0.8$ mmol L $\mathrm{L}^{-1}$, respectively. ${ }^{54}$ Note that this enhancement is considerably larger than the expected decrease in apparent ${ }^{1} \mathrm{O}_{2}$ quantum yields due to change in $\mathrm{O}_{2}$ content (see above).

Despite of these observations, a convincing explanation on the photophysics of this enhancement is still lacking. Work by Parker et al. ${ }^{223}$ showed that halides enhance triplet steady-state concentrations by increasing ${ }^{3} \mathrm{CDOM}^{*}$ lifetimes (i.e., decreasing $\left.k_{\mathrm{d}}^{\mathrm{T}}\right)$, which might result in an increase in $\Phi_{\Delta}$ based on eq 2 . However, many points remain to be to clarified, including the heavy atom effect (i.e., larger enhancement for $\mathrm{Br}^{-}$compared to $\mathrm{Cl}^{-}$when present at the same concentrations), which was observed in $\Phi_{\Delta}$ measurements but not for ${ }^{3} \mathrm{CDOM}^{*}{ }^{54,223}$ Further studies need to confirm the role of halides in increasing apparent ${ }^{1} \mathrm{O}_{2}$ quantum yields, which would allow better understanding the origin of the overall higher $\Phi_{\Delta}$ in marine environments as compared to freshwater systems (section 4.2.4).

Concerning other ionic species, Paul et al. ${ }^{83}$ noted a strong positive correlation between $\Phi_{\Delta}$ and nitrate $(r=0.875)$ for ten humic acid samples collected in Scandinavia. In this study, apparent ${ }^{1} \mathrm{O}_{2}$ quantum yields were obtained via time-resolved phosphorescence; thus, artifacts related to ${ }^{\circ} \mathrm{OH}$-induced degradation of the ${ }^{1} \mathrm{O}_{2}$ probe can be excluded (see also section 3.2.2.4). On the other hand, there is evidence that phosphate (up to $30 \mathrm{mmol} \mathrm{L}^{-1}$ ) and carbonate (up to $2 \mathrm{mmol}$ $\mathrm{L}^{-1}$ ) do not affect apparent ${ }^{1} \mathrm{O}_{2}$ quantum yields. ${ }^{47}$ Similarly, no effect on $\Phi_{\Delta}$ was noted upon changing the solvent from $\mathrm{H}_{2} \mathrm{O}$ to $\mathrm{D}_{2} \mathrm{O}$ in steady-state determinations based on probe compounds. $^{71}$

Wan and coauthors ${ }^{79}$ investigated the effect of metal ions on apparent quantum yields of various photochemically produced reactive intermediates, including ${ }^{1} \mathrm{O}_{2}$, and found a strong 
Table 6. Summary of the Most Relevant Correlations between Apparent ${ }^{1} \mathrm{O}_{2}$ Quantum Yields and Molecular, Optical, and Photochemical DOM Features

\begin{tabular}{|c|c|c|c|}
\hline parameter & symbol & correlation $^{a}$ & refs \\
\hline \multicolumn{4}{|l|}{ molecular features } \\
\hline average molecular weight ${ }^{b}$ & MW & -- & $33,37,55-57,60,74,77$ \\
\hline electron donating capacity & EDC & - & 40,42 \\
\hline electron accepting capacity & EAC & - & 40,42 \\
\hline antioxidant capacity & $\mathrm{A}_{x} \mathrm{~A}_{50}$ & - & 35 \\
\hline organic carbon content & {$[\mathrm{DOC}]$} & n.s. & $34,37,39,40,75$ \\
\hline aromatic ketones contribution & & ++ & 63 \\
\hline weighted C-normalized double bond equivalents & $\mathrm{DBE} / \mathrm{C}_{\mathrm{avg}}$ & -- & 76,77 \\
\hline weighted $\mathrm{H}: \mathrm{C}$ ratio & $\mathrm{H}: \mathrm{C}_{\text {avg }}$ & ++ & 76,77 \\
\hline \multicolumn{4}{|l|}{ optical features } \\
\hline E2:E3 & $\mathrm{E} 2: \mathrm{E} 3$ & ++ & $32,34-36,40,43,48,53,55-57,60,61,63,71,74-77,79$ \\
\hline spectral slope & $S_{300-600}, S_{275-295}$ & ++ & $32,35,36,43,57$ \\
\hline spectral slope ratio & $S_{\mathrm{R}}$ & n.s. to ++ & $32,35,53$ \\
\hline specific ultraviolet absorbance at $254 \mathrm{~nm}$ & SUVA $_{254}$ & - & $32,34,35,43,75,77$ \\
\hline fluorescence index & FI & + & 32,57 \\
\hline humification index & HIX & - & 32,35 \\
\hline biological fluorescence index & BIX & + & 32 \\
\hline \multicolumn{4}{|l|}{ photophysical features } \\
\hline triplet quantum yield coefficient & $f_{\mathrm{TMP}}$ & ++ & $35,63,77$ \\
\hline fluorescence quantum yield & $\Phi_{\mathrm{F}}$ & + & 43 \\
\hline
\end{tabular}

${ }^{a}$ Legend: $(--)$, strong negative correlation; $(-)$, weak negative correlation; n.s., nonsignificant correlation; $(+)$, weak positive correlation; $(++)$, strong positive correlation. These classifications should be considered only qualitative and indicative; see text for more details. ${ }^{b}$ Only direct average molecular weight measurements.

negative correlation between $\Phi_{\Delta}$ and $\log K_{\mathrm{ML}}\left(K_{\mathrm{ML}}\right.$ is the stability constant for complex formation between the metal ions and DOM). In particular, they observed that strongly bound paramagnetic metals, such as $\mathrm{Fe}^{3+}$ and $\mathrm{Cu}^{2+}$, result in more significant static and dynamic ${ }^{3} \mathrm{CDOM}^{*}$ quenching, which in turn reduces apparent ${ }^{1} \mathrm{O}_{2}$ quantum yields. This effect is also DOM dependent, as humic acids have a stronger metal binding ability compared to fulvic acids and effluent organic matter. To the authors' knowledge, the effect of metal content on apparent ${ }^{1} \mathrm{O}_{2}$ quantum yields under environmentally relevant conditions has not yet been investigated.

\subsection{Molecular, Optical, and Photophysical DOM Features}

5.2.1. Macroscopic and Microscopic Molecular Features. Average DOM molecular weight appears to be the primary parameter that explains most of the variations reported in $\Phi_{\Delta}$ values. Indeed, most of the trends with spectroscopic parameters such as E2:E3 or spectral slopes are ultimately related to trends in molecular weight (section 5.2.2). Similarly, variations in quantum yields as a function of DOM origin (i.e., autochthonous vs allochthonous) and subfractions (i.e., fulvic acids vs humic acids) can also be interpreted as due to differences in molecular weight. Quantum yields of other reactive intermediates and fluorescence quantum yields are also correlated to molecular weight (section 5.2.3), while effects of oxidative treatments of organic matter are typically explained in terms of changes in the average molecular size (section 5.3.2). In addition, several authors directly analyzed the impact of the size distribution on $\Phi_{\Delta}$ using a variety of techniques including gel permeation chromatography, ${ }^{33}$ size exclusion chromatography, ${ }^{57}$ ultrafiltration, ${ }^{37,53-57,60,74}$ and high-resolution mass spectrometry (HRMS) ${ }^{77}$ and confirmed the presence of a negative correlation between molecular weight and apparent ${ }^{1} \mathrm{O}_{2}$ quantum yield in a wide range of DOM samples. McKay and coauthors ${ }^{60}$ obtained similar results using chemical size fractionation techniques such as flocculation via base modification and coagulation with alum (aluminum sulfate decahydrate), two common wastewater treatment strategies.

Despite this large body of evidence, the photophysical basis of the correlation between $\Phi_{\Delta}$ and molecular weight is still under debate. One popular explanation builds on the charge transfer (CT) model, ${ }^{28,214}$ which has been used to justify several optical and photochemical properties of DOM (even if recent work has triggered discussions on the validity of this interpretation $\left.{ }^{224-226}\right)$. According to this model, a significant portion of DOM absorbance is due to the presence of charge transfer interactions between donors ( $D$, proposed to be phenols and methoxyphenols) and acceptors (A, proposed to be quinones, aromatic ketones, and other aromatic carbonyls). Both donors and acceptors may be formed through oxidation of lignin-derived precursors. In the presence of $\mathrm{D}-\mathrm{A}$ interactions, excited states of $\mathrm{CDOM}$ (both ${ }^{1} \mathrm{CDOM}^{*}$ and ${ }^{3} \mathrm{CDOM}^{*}$ ) can be deactivated via their conversion to chargeseparated complexes $\left(\mathrm{CDOM}^{+\bullet /-\bullet}\right)$ that are not photochemically active, which results in a decrease in fluorescence and triplet quantum yields. In turn, a decrease in $\Phi_{\mathrm{T}}$ leads to a reduction of ${ }^{1} \mathrm{O}_{2}$ production, and therefore in a decrease in $\Phi_{\Delta}$. The current understanding is that larger molecules engage in more CT interactions than smaller compounds, resulting in lower triplet and singlet oxygen quantum yields. ${ }^{57,60,74}$

A second explanation is that lower-molecular-weight fractions are better photosensitizers because of their reduced absorption compared to high molecular weight fractions, and not because they inherently produce more ${ }^{1} \mathrm{O}_{2} \cdot{ }^{33,55,57,74}$ This interpretation is supported by the changes in the absorption spectra of size-fractionated DOM compared to whole water samples, which typically show decreased absorbance for lowmolecular-weight fractions and increased absorbance for highmolecular-weight fractions as compared to whole waters. $^{57,74,227,228}$ Mostafa and Rosario-Ortiz. ${ }^{55}$ proposed two 
additional interpretations for the molecular weight trends. The first builds on the work of Latch and McNeill, ${ }^{196}$ who observed significantly higher singlet oxygen steady-state concentrations within the DOM microenvironment as compared to the bulk of the solution. The authors proposed that more ${ }^{1} \mathrm{O}_{2}$ can escape the hydrophobic microenvironment in low-molecularweight fractions due to decreased self-quenching, which results in artificially higher "bulk" singlet oxygen concentrations. Even if reasonable, this hypothesis has never been tested experimentally. The second interpretation is based on the observation that high-molecular-weight compounds have typically a higher degree of conjugation. Increased conjugation results in the formation of low energy singlet and triplet excited states, which have low lifetimes due to an increased likelihood of decay via nonradiative pathways.

A few studies analyzed the correlation between apparent ${ }^{1} \mathrm{O}_{2}$ quantum yields and redox active moieties quantified in terms of electron donating or accepting capacity (EDC and EAC, respectively $)^{40,42}$ or antioxidant activity $\left(\mathrm{A}_{x} \mathrm{~A}_{50}\right){ }^{35} \mathrm{EDC}$ and EAC are measured via mediated electrochemical oxidation, and are indicative of the concentration of phenol and quinone moieties, respectively. ${ }^{229}$ The antioxidant activity is obtained via the $\mathrm{DPPH}^{\bullet}$ (2,2-diphenyl-1-picrylhydrazyl) assay, and it is linearly correlated with EDC according to the equation $\mathrm{A}_{x} \mathrm{~A}_{50}$ $=17.48$ EDC $-0.12 .{ }^{230}$ Apparent ${ }^{1} \mathrm{O}_{2}$ quantum yields displayed negative correlations with $\mathrm{EDC}, \mathrm{A}_{x} \mathrm{~A}_{50}, \mathrm{EAC}$, and the total redox capacity $(\mathrm{EDC}+\mathrm{EAC}) .^{35,40,42}$ McKay et al. ${ }^{35}$ found a linear correlation between antioxidant activity and $\Phi_{\Delta}$ only for $A_{x} A_{50}$ between 5 and 20; for larger ranges of $A_{x} A_{50}$ values (up to 50 ), a strong positive linear correlation was found between $1 / \Phi_{\Delta}$ and $A_{x} A_{50}$ instead. Correlations between apparent singlet oxygen quantum yields and EDC or $\mathrm{A}_{x} \mathrm{~A}_{50}$ are interpreted as due to a combination of $\mathrm{CT}$ interactions and intramolecular quenching. ${ }^{35,42}$ More specifically, high EDC is indicative of high concentrations of electron donors (i.e., phenols) that can engage in CT interactions. Thus, the higher the concentration of donors, the higher the probability of CT interactions, and hence, the lower the apparent singlet oxygen quantum yields. On the other hand, phenols are also efficient triplet quenchers, and, similar to CT interactions, can lower $\left[{ }^{3} \mathrm{CDOM}^{*}\right]_{\mathrm{ss}}$ by reacting with triplets. Again, suppressed triplet concentrations would result in a decrease in $\Phi_{\Delta} \cdot{ }^{35,42}$ On the other hand, Sharpless et al. $^{42}$ interpreted the correlation between $\Phi_{\Delta}$ and EAC or (EAC+EDC) as evidence of the important role of redox-active moieties in DOM photochemistry.

Zhou et al. ${ }^{63}$ found positive correlations between apparent ${ }^{1} \mathrm{O}_{2}$ quantum yields and aromatic ketone content $\left(\mathrm{R}^{2}=0.87 ; \mathrm{N}\right.$ $=10)$ for IHSS isolates and hydrophobicity-fractionated effluent organic matter samples, which further strengths the hypothesis that aromatic ketones are implicated in the formation of ${ }^{1} \mathrm{O}_{2}$. In their work, the authors quantified the contribution of (reducible) aromatic ketones based on the decrease of the FFA degradation rate constant upon $\mathrm{NaBH}_{4}$ reduction. In addition, Paul and coauthors ${ }^{83}$ reported a positive trend $(r=0.818)$ between the FT-IR absorbance between 1000 and $1060 \mathrm{~cm}^{-1}$ (relative to polysaccharides and methoxy groups) and the apparent ${ }^{1} \mathrm{O}_{2}$ quantum yields of twenty-seven humic substances isolated from soil and freshwater sources. On the other hand, $\mathrm{Du}$ et al. ${ }^{32}$ tried to use ${ }^{1} \mathrm{H}$ NMR indexes (i.e., percent composition of methyl, aliphatic, aromatic, etc.) to produce a multiple linear regression model for $\Phi_{\Delta}$ but found that these parameters have a poor predictive power as compared to spectroscopic features such as E2:E3 or spectral slopes (see section 5.2.2). The authors justify this result in terms of the $\mathrm{CT}$ model, pointing out that ${ }^{1} \mathrm{H}$ NMR indexes cannot quantify accurately donor-acceptor interactions. $^{32}$

Remucal's group went further to analyze correlations between apparent quantum yields and microscopic molecular parameters quantified via Fourier-transform ion cyclotron resonance mass spectrometry (FT-ICR-MS) and found results in agreement with standard bulk measurements. More specifically, they observed that $\Phi_{\Delta}$ (and $\Phi_{\mathrm{T}}$ ) correlates positively with the weighted $\mathrm{H}: \mathrm{C}$ average $\left(\mathrm{H}: \mathrm{C}_{\text {avg }} ; R^{2}=0.98\right.$, $N=7$ ), and negatively with the C-normalized average double bond equivalent $\left(\mathrm{DBE} / \mathrm{C}_{\text {avg }} ; \mathrm{R}^{2}=0.94, N=7\right.$ ) in $\mathrm{DOM}$ samples collected in seven lakes of different trophic status in North Wisconsin, US. ${ }^{76}$ These two parameters are directly related to bulk DOM aromaticity as quantified by $\mathrm{SUVA}_{254}$, with $\mathrm{H}: \mathrm{C}_{\mathrm{avg}}$ showing a negative and $\mathrm{DBE} / \mathrm{C}_{\text {avg }}$ a positive linear correlation, respectively. They also looked for relationships between the relative intensity of individual $\mathrm{CHON}_{0-1}$ molecular formulas and triplet and ${ }^{1} \mathrm{O}_{2}$ quantum yields, and found positive correlations for less aromatic and less oxidized formulas (i.e., more saturated). ${ }^{76}$ In addition, they observed that $97 \%$ of the formulas that correlated with $\Phi_{\mathrm{T}}$ are also correlated with E2:E3, ${ }^{76}$ demonstrating that there is a molecular justification for the well-known trend between $\Phi_{\mathrm{T}}$ (thus, $\Phi_{\Delta}$ ) and E2:E3 observed in bulk measurements (see section 5.2.2). These results were further corroborated by a following publication that used DOM samples collected along the St. Louis river. ${ }^{77}$

Several authors reported minimal effects of increasing DOM concentrations on $\Phi_{\Delta}$ when working in $\mathrm{H}_{2} \mathrm{O} \cdot{ }^{34,37,39,40,75}$ In particular, Partanen and coauthors ${ }^{39}$ saw a linear response of the ${ }^{1} \mathrm{O}_{2}$ phosphorescence signal in $\mathrm{H}_{2} \mathrm{O}$ for concentrations between 17.5 and $350 \mathrm{mg} \mathrm{L}^{-1}$, and negligible variations in $\Phi_{\Delta}$ for different samples and excitation wavelengths using DOM concentrations between 70 and $700 \mathrm{mg} \mathrm{L}^{-1}$. These observations agree with our calculations that showed negligible ${ }^{1} \mathrm{O}_{2}$ quenching by DOM in $\mathrm{H}_{2} \mathrm{O}$ even for very high [DOC]; however, quenching becomes significant when switching to $\mathrm{D}_{2} \mathrm{O}$ (section $\mathrm{S} 1$ and Figure $\mathrm{S} 1$ ).

5.2.2. Optical Features. The most popular spectroscopic index for predicting apparent ${ }^{1} \mathrm{O}_{2}$ quantum yields is E2:E3, which is defined as the ratio of the absorbance at 254 or at 250 $\mathrm{nm}$ to that at $365 \mathrm{~nm} \cdot{ }^{45,214,231} \mathrm{E} 2: \mathrm{E} 3$ is known to be (strongly) negatively correlated with the average DOM molecular weight, with lower E2:E3 values for high-molecular-weight material. $^{44,231}$ Several studies reported a strong positive (linear) correlation between E2:E3 and $\Phi_{\Delta}$ (see Table 6 for refs), implying that DOM with low average molecular weight is more efficient in producing ${ }^{1} \mathrm{O}_{2}$. Based on this observation, several authors proposed the use of E2:E3 as a predictor of apparent ${ }^{1} \mathrm{O}_{2}$ quantum yields and provided linear equations to be used in models (Table 7). A literature analysis by $\mathrm{Du}$ and coworkers $^{32}$ showed that the relationship between $\Phi_{\Delta}$ and E2:E3 holds valid when comparing values for different DOM types and studies (for the same irradiation source), suggesting a common photophysical base for this correlation. At least two studies have explored multiple linear regression models for the prediction of $\Phi_{\Delta}$ from spectroscopic and molecular parameters but found that including more information did not improve the predictions performed with E2:E3 alone. ${ }^{32,35}$ This result was 
Table 7. Summary of Parameters Relative to the Regression Line $\Phi_{\Delta}=a(\mathrm{E} 2: \mathrm{E} 3)+b^{a}$

\begin{tabular}{|c|c|c|c|c|c|}
\hline reference & slope & intercept & $R^{2}$ & E2:E3 range & $N$ \\
\hline \multicolumn{6}{|l|}{ xenon lamp (solar) } \\
\hline Mostafa and Rosario-Ortiz, 2013 & 0.72 & 0.20 & $0.56^{\dagger}$ & $3.1-11.1^{\S}$ & 23 \\
\hline Cawley et al., $2014^{\dagger}$ & $2.2 \pm 0.4$ & $-(7 \pm 2)$ & 0.97 & $4.2-6.0^{\S}$ & 3 \\
\hline Mostafa et al., 2014 & $1.20 \pm 0.12$ & $-(2.9 \pm 0.6)$ & 0.94 & $3.2-7.8^{\S}$ & 8 \\
\hline McCabe and Arnold, 2016 & 0.62 & -0.11 & 0.83 & $4.3-16.3$ & 45 \\
\hline McKay et al., 2016 & 1.59 & -5.29 & 0.73 & $4.5-6$ & 14 \\
\hline McKay et al., 2017 & 0.64 & n.s. & 0.69 & $5.3-16.9$ & 22 \\
\hline Silva et al., $2017^{\dagger}$ & $0.08 \pm 0.06$ & $0.64 \pm 0.17$ & 0.64 & $2.4-3.6^{\S}$ & 3 \\
\hline Zhou et al., 2017 & $b$ & $b$ & 0.95 & $3.4-6.2^{\S}$ & 12 \\
\hline Du et al., 2018 & 0.63 & -0.19 & 0.78 & $2.7-9.7^{\S}$ & 18 \\
\hline Leresche et al., $2019^{\dagger c}$ & $0.63 \pm 0.05$ & $0.008 \pm 0.640$ & 0.71 & $4.8-24.2$ & 58 \\
\hline Wang et al., $2019^{\dagger}$ & $0.24 \pm 0.19$ & $1.7 \pm 1.0$ & 0.42 & $2.2-4.4$ & 4 \\
\hline average & $0.86 \pm 0.64$ & $-(0.7 \pm 3.0)$ & & & \\
\hline \multicolumn{6}{|c|}{ UVA (mercury, xenon with filter, Rayonet lamps) } \\
\hline Dalrymple et al., 2010 & 0.87 & -1.53 & 0.65 & $3.1-7.0^{\S}$ & 18 \\
\hline Peterson et al., 2012 & 0.20 & 0.61 & 0.59 & $4.7-21.4$ & 50 \\
\hline Sharpless, $2012^{d}$ & $\mathrm{n} / \mathrm{a}$ & $\mathrm{n} / \mathrm{a}$ & $\mathrm{n} / \mathrm{a}$ & $\mathrm{n} / \mathrm{a}$ & $\mathrm{n} / \mathrm{a}$ \\
\hline Bodhipaksha et al., $2015^{\dagger}$ & $1.7 \pm 0.3$ & $-(5.7 \pm 1.3)$ & 0.73 & $3.9-7.2^{\S}$ & 20 \\
\hline Maizel and Remucal, 2017 (ESPI) $)^{\dagger e}$ & $0.05 \pm 0.04$ & $1.0 \pm 0.2$ & 0.12 & $4.7-10.5$ & 16 \\
\hline Maizel and Remucal, 2017 (ES\&T) $)^{\dagger, c}$ & $1.2 \pm 0.3$ & $-(3.8 \pm 1.3)$ & 0.72 & $3.8-5.9$ & 10 \\
\hline Maizel et al., 2017 & 0.22 & 0.42 & 0.91 & $4.5-9.8$ & 7 \\
\hline Berg et al., 2019 & $0.18 \pm 0.05$ & $-(0.16 \pm 0.23)$ & 0.72 & $4.2-6.3$ & 10 \\
\hline Zhou et al., 2020 & 0.52 & -0.8 & 0.91 & $3.0-8.1^{\S}$ & 7 \\
\hline average & $0.62 \pm 0.59$ & $-(1.2 \pm 2.4)$ & & & \\
\hline
\end{tabular}

$a_{a}$ is the slope and $b$ is the intercept of the linear regression. When not specified, E2 is the absorbance at 250 nm; authors that used the absorbance at $254 \mathrm{~nm}$ are indicated with the symbol $\S$. In some cases, the regression parameters were not reported in the studies and were calculated using the data from the database (indicated as $\dagger$; errors are standard errors of the regression parameters). ${ }^{b}$ Exponential fit. ${ }^{c}$ Overall slope considering SRFA and PLFA together. ${ }^{d}$ E2:E3 data not available. ${ }^{e}$ All data from ambient conditions and SPE isolate conditions included. A stronger regression was observed when wastewater effluents were excluded from the fit. ${ }^{75}$ Legend: n.s., nonsignificant; n/a, not available.

partly explained by the presence of intercorrelations between the parameters used for the regression.

However, there are still a few points to be clarified before adopting this spectral parameter as a robust quantum yield predictor. In particular, there is a lack of consensus on slope and intercept of the linear regression equation, as hinted by the range of values reported in the literature also for the same light source (Table 7). Furthermore, McKay et al. ${ }^{35}$ pointed out that the slope changes significantly as a function of the range of E2:E3 values used in the regression. In their analysis, they obtained a slope of $1.16 \pm 0.18$ and $0.661 \pm 0.035$ using literature values with E2:E3 between $3.1-6$ and 3.1-16.9, respectively. Interestingly, this bimodal behavior was not evident when using the spectral slope as the $\Phi_{\Delta}$ predictor. $^{35}$ On a similar line, Zhou et al. ${ }^{63}$ found an exponential (instead of linear) increase of $\Phi_{\Delta}$ as a function of E2:E3 for IHSS isolates and whole and fractionated effluent organic matter samples (E2:E3 = 3.4-6.2). Other authors suggested that slopes are not only dependent on E2:E3, but also on the DOM type, ${ }^{42,60,74}$ the degree of oxidation, ${ }^{42,55}$ and on the use of whole water samples vs isolates. ${ }^{36} \mathrm{We}$ also highlight that some authors calculated E2:E3 using the absorbance at $250 \mathrm{~nm}$, while others used the value at $254 \mathrm{~nm}$ (Table 7). Albeit minimal, this inconsistency might explain some of the variability observed in slopes and intercepts.

Several studies also reported strong positive correlations between apparent ${ }^{1} \mathrm{O}_{2}$ quantum yields and spectral slopes. ${ }^{32,35,36,43,57}$ Spectral slopes are calculated by fitting absorption spectra (in Napierian units) with an exponential decay function over a well-defined wavelength range, typically
$275-295 \mathrm{~nm}\left(S_{275-295}\right)$ or $300-600 \mathrm{~nm}\left(S_{300-600}\right) \cdot{ }^{44,231}$ A few authors also reported correlations between $\Phi_{\Delta}$ and the spectral slope ratio $\left(S_{\mathrm{R}}\right){ }^{32,35,53}$ which is defined as $S_{275-295} / S_{350-400 \cdot}{ }^{231}$ Similarly to E2:E3, spectral slope parameters correlate negatively with molecular weight, resulting in higher $S$ (or $S_{\mathrm{R}}$ ) for lower-molecular-weight material. ${ }^{214,231}$ Both $S_{275-295}{ }^{32}$ and $S_{300-600} 35,36,43,57$ (but not $\left.S_{350-400}\right)^{32}$ are good $\Phi_{\Delta}$ predictors, and, for the same data set, they often provide stronger correlations than with E2:E3. ${ }^{35,36}$ On the other hand, authors found strong $\left(R^{2}=0.93\right),{ }^{53} \operatorname{good}\left(R^{2}=0.43\right),{ }^{32}$ and nonsignificant ${ }^{35}$ correlations between $S_{R}$ and $\Phi_{\Delta}$.

Correlations between E2:E3, spectral slopes, and $\Phi_{\Delta}$ are also typically justified in terms of charge transfer (CT) interactions. ${ }^{32,214}$ In particular, both E2:E3 and spectral slopes are thought to be indicative of the number of charge transfer contacts. Soil humic acids and terrestrially derived DOM, which have high lignin content, are typically interpreted as having a high degree of CT interactions, which is reflected in low E2:E3 and $S$ values. ${ }^{214}$ Increasing charge transfer interactions are theorized to result in low $\Phi_{\Delta}$ according to the mechanism explained above. Furthermore, CT contacts are probabilistically more abundant in high-molecular-weight fractions, which explains the correlation between these optical parameters and average molecular weight.

Several studies looked for trends between apparent ${ }^{1} \mathrm{O}_{2}$ quantum yields and other spectroscopic parameters, but correlations were not as strong as with E2:E3 or spectral slopes. For example, several authors found negative correlations between $\Phi_{\Delta}$ and $\mathrm{SUVA}_{254}, 32,34,35,43,75,77,77$ the DOCnormalized absorbance at $254 \mathrm{~nm}, \mathrm{SUVA}_{280},{ }^{32} \mathrm{SUVA}_{300}{ }^{35}$ and 
$\mathrm{SUVA}_{400} \cdot{ }^{83}$ In agreement with the framework above, $\mathrm{SUVA}_{254}$ increases with average molecular weight and aromaticity; thus, higher $\mathrm{SUVA}_{254}$ values are associated with lower $\Phi_{\Delta}$ due to increasing average molecular weight. A couple of authors also reported (weak) positive correlations with the fluorescence index $(\mathrm{FI})^{32,57}$ and the biological fluorescence index (BIX), ${ }^{32}$ and negative correlations with the humification index (HIX). ${ }^{32,35}$ All of these relationships can be qualitatively explained by the higher apparent ${ }^{1} \mathrm{O}_{2}$ quantum yields of autochthonous DOM (high FI and BIX, low HIX) as compared to terrestrial DOM (low FI and BIX, high HIX).

5.2.3. Photophysical Features. Given that triplet excited states of CDOM are the direct precursors of singlet oxygen (Figure 2), correlations between the formation yields of these two species are expected. Indeed, apparent singlet oxygen quantum yields are strongly positively correlated with the triplet quantum yield coefficient $\left(f_{\mathrm{TMP}}\right){ }^{35,63,77}$ with reported $R^{2}$ values close to $\sim 0.9 .^{35,63}$ The triplet quantum yield coefficient is calculated as the ratio of the observed firstorder rate constant of 2,4,6-trimethylphenol (TMP, the probe compound for $\left.{ }^{3} \mathrm{CDOM}^{*}\right)^{155}$ with CDOM and the rate of light absorption, and is directly proportional to $\Phi_{\mathrm{T}} \cdot{ }^{75}$ This strong correlation is not only consistent with the fact that ${ }^{3} \mathrm{CDOM}^{*}$ is the precursor of ${ }^{1} \mathrm{O}_{2}$ (Figure 2), but it also implies that a very similar DOM pool is responsible for both the oxidation of TMP and the formation of ${ }^{1} \mathrm{O}_{2} .{ }^{77}$ Consistent with this view, several authors reported strong positive correlations between $f_{\text {TMP }}$ and E2:E3 or spectral slope parameters, ${ }^{76,77}$ and negative correlations between $f_{\text {TMP }}$ and SUVA $_{254},{ }^{77}$ exactly as it is observed for $\Phi_{\Delta}$. For this reason, McKay et al. ${ }^{35}$ proposed to use apparent ${ }^{1} \mathrm{O}_{2}$ quantum yields as a predictor of ${ }^{3} \mathrm{CDOM}^{*}$ reactivity, a more challenging oxidant to characterize compared to singlet oxygen due to its heterogeneous nature. ${ }^{5}$

Leresche and coauthors ${ }^{43}$ observed a positive correlation between $\Phi_{\Delta}$ and the fluorescence quantum yield $\left(\Phi_{\mathrm{F}}\right)$ for two organic matter isolates treated with increasing levels of ozone. They also noted that this relationship was valid only for relatively low quantum yield values, and they interpreted this result as an indication that there are two distinct CDOM pools responsible for fluorescence and ${ }^{1} \mathrm{O}_{2}$ generation. ${ }^{43}$

\subsection{Chemical Treatments}

5.3.1. Reductive Treatments. Reduction of DOM with $\mathrm{NaBH}_{4}$ has been conducted with the goal of examining the role of aromatic ketones in ${ }^{1} \mathrm{O}_{2}$ production and to test the validity of the CT model. ${ }^{59,60,63,71}$ In these experiments, DOM was first treated with $\mathrm{NaBH}_{4}$ under anoxic conditions to reduce aromatic ketones, aldehydes, and quinones to the corresponding alcohols and hydroquinones. In a second step, the DOM solution was reoxygenated to convert hydroquinones back to quinones and to eliminate the excess of $\mathrm{NaBH}_{4}{ }^{232}$ These borohydride reduction experiments have consistently shown a decrease in visible absorption, which increases E2:E3, and an increase and a blue-shift in DOM fluorescence, all empirical observations that support the charge transfer model. ${ }^{214,232}$ The spectroscopic data combined with the extensive prior literature on borohydride reduction support the loss of ketones, which have been hypothesized as being important ${ }^{1} \mathrm{O}_{2}$ sensitizers. However, these studies typically report no or minimal decrease in apparent ${ }^{1} \mathrm{O}_{2}$ quantum yields in diverse organic matter samples, including IHSS isolates, effluent organic matter isolates, and dissolved black carbon. ${ }^{59,60,63,71}$ Similar results were obtained for TMP reactivity, indicating little change in quantum yield efficiency of ${ }^{3} \mathrm{CDOM}^{*}$ production. ${ }^{63,71}$ This result fits with the idea that there is a good overlap between the ${ }^{3} \mathrm{CDOM}^{*}$ pool that can oxidize TMP and the one that forms ${ }^{1} \mathrm{O}_{2} \cdot{ }^{71}$

Interpreting the quantum yield invariance following borohydride reduction is challenging, as there are different possible explanations. It could be that borohydride-reactive ketones and borohydride-unreactive moieties both have similar quantum yields, such that the removal of ketones does not change the efficiency of ${ }^{1} \mathrm{O}_{2}$ production. Sharpless ${ }^{42}$ hypothesized that, instead of two chemically distinct pools, there is a single pool of sensitizers that cannot be completely reduced by $\mathrm{NaBH}_{4}$ (for example, because of electrostatic repulsion between $\mathrm{BH}_{4}^{-}$and $\mathrm{DOM}$ at high $\mathrm{pH}$ ). Sharpless interpreted the decrease in UV-vis absorption upon reduction as due to a change in sensitizers' concentration, which does not affect $\Phi_{\Delta}$ because the decrease in absorbance is proportional to the suppression in ${ }^{1} \mathrm{O}_{2}$ production. This interpretation is consistent with the view that most of the visible absorption of DOM involves chromophores with ${ }^{1} \mathrm{O}_{2}$ sensitizing abilities (i.e., aromatic ketones), and it is in line with the CT model, where visible absorbance comes from CT interactions involving ketones as acceptors. Other explanations involving two or more pools of sensitizers have been put forward to justify the invariance or the decrease in $\Phi_{\Delta}$ and its relationships with trends in TMP reactivity; some authors also hypothesized that $\mathrm{NaBH}_{4}$ reduction might alter the rates of ${ }^{1} \mathrm{O}_{2}$ loss within the DOM microenvironment. ${ }^{60,63,71}$ At the present moment, all of the above explanations are highly speculative and lack convincing empirical confirmations.

5.3.2. Oxidative Treatments. Oxidative treatments include photooxidation, ozonation, and oxidation with hypochlorous acid ( $\mathrm{HOCl}) .^{42,43,55}$ To date, Sharpless and coauthors ${ }^{42}$ performed the most systematic study on the effects of photooxidation on apparent quantum yields of PPRIs, including ${ }^{1} \mathrm{O}_{2}$. In this work, they observed a net increase of $\Phi_{\Delta}$ for SRFA, Nordic Aquatic FA, and Elliot soil HA upon $59 \mathrm{~h}$ of solar irradiation, while apparent ${ }^{\bullet} \mathrm{OH}$ quantum yields and $f_{\text {TMP }}$ decreased. ${ }^{42}$ Concomitant with these changes, they reported a decrease in molecular weight, EDC, and $\mathrm{SUVA}_{280}$, and an increase in E2:E3. To reconcile the simultaneous decrease in $\Phi_{\Delta}$ and increase in $f_{\mathrm{TMP}}$ for all three isolates, the authors posited that photooxidation has the net effect of decreasing ${ }^{1} \mathrm{O}_{2}$ quenching (via electron transfer) in the DOM microenvironment. This interpretation is supported by the observation of a strong decrease in EDC upon oxidation, which indicates loss of phenolic moieties, thus electron transfer quenchers. In their view, this explanation does not contradict other reports that found a large overlap between the ${ }^{3} \mathrm{CDOM}^{*}$ pool responsible for ${ }^{1} \mathrm{O}_{2}$ formation and the one responsible for TMP oxidation. ${ }^{70,71}$ A second hypothesis is that there exist two pools of ${ }^{1} \mathrm{O}_{2}$ sensitizers, one photostable (i.e., quinones), and one photolabile (i.e., aromatic ketones). The photolabile pool is partially responsible for UVA and visible light absorption and it is selectively removed during photooxidation, justifying the trends in optical parameters. This rational is also in agreement with the minimal changes in EAC (quantifying the amount of quinones) upon photooxidation, and the fact that Elliot soil HA, which has a larger proportion of quinones as compared to aquatic fulvic acids, showed the largest increase in $\Phi_{\Delta}$. 
Other authors invoked photooxidation to explain seasonal trends in apparent ${ }^{1} \mathrm{O}_{2}$ quantum yields (discussed also in section 5.5), but several points remain to be clarified. For example, Paul and coauthors ${ }^{83}$ explained the decrease in $\Phi_{\Delta}$ observed between spring and autumn in humic acids isolated from Scandinavian lakes as due to photochemical degradation occurring during the summer months. We should note that this work was published well before Sharpless et al. ${ }^{42}$ showed that photodegradation increases apparent ${ }^{1} \mathrm{O}_{2}$ quantum yields. Thus, the experimental observations by Paul and coauthors indicate that factors other than photodegradation control the seasonal dynamics of ${ }^{1} \mathrm{O}_{2}$ in these boreal lakes. On the other hand, McCabe and Arnold ${ }^{34}$ detected an increase in $\Phi_{\Delta}$ for DOM isolated from different types of Prairie Pothole lakes throughout the growing season, and hypothesized that photodegradation was responsible for this trend. Nevertheless, they also reported an increase in apparent quantum yields for other PPRIs, which is in contrasts with the result from Sharpless et al. $^{42}$ and weakens the hypothesis that photodegradation is the only driver of seasonal change in DOM photoreactivity in this environment.

Similar to photodegradation, ozonation and chlorination were also found to increase $\Phi_{\Delta}$ of both effluent organic matter and aquatic fulvic acids. ${ }^{43,55}$ Consistently, these oxidative treatments decreased the overall absorbance, $\mathrm{SUVA}_{254}$, and increased E2:E3 and the spectral slope, even if the magnitude of the changes varied based on the oxidant. ${ }^{43,55}$ Early work from Mostafa and Rosario-Ortiz ${ }^{55}$ interpreted these variations in terms of changes in average molecular weight, a parameter that is well-known to be inversely correlated to apparent ${ }^{1} \mathrm{O}_{2}$ quantum yields (section 5.2.1). Other explanations that have been put forward involve the presence of two pools of sensitizers, and a reduced ${ }^{1} \mathrm{O}_{2}$ quenching efficiency upon oxidation, $^{55}$ similar to what Sharpless and coauthors ${ }^{42}$ proposed to explain trends of $\Phi_{\Delta}$ as a function of photooxidation. More recently, Leresche et al. ${ }^{43}$ suggested that the increase in apparent ${ }^{1} \mathrm{O}_{2}$ quantum yields upon ozonation is caused by the formation of quinone-like moieties. Ozone is known to be a selective oxidant that converts phenols into quinones, with a product distribution that depends on the solution $\mathrm{pH}^{233-236}$ In their work, the authors observed a dramatic increase in $\Phi_{\Delta}$ for both SRFA and PLFA upon treatment with up to $1 \mathrm{mmol}_{\mathrm{O} 3} \mathrm{mmol}_{\mathrm{C}}{ }^{-1}$, with different trends depending on the ozonation $\mathrm{pH} .{ }^{43}$ For instance, for PLFA at $\mathrm{pH} 3, \Phi_{\Delta}$ increased from $2.9 \%$ to $13.1 \%$, while, at $\mathrm{pH} 7$, it raised from $2.7 \%$ to $17.0 \%$. They further noted that quantum yields in the tens of percent were also measured in fog waters collected in two Californian cities, ${ }^{8}$ and speculated that atmospheric ozone oxidation might justify the high $\Phi_{\Delta}$ of these atmospheric samples. The latter is an intriguing hypothesis that has yet to be tested.

\subsection{Wavelength Dependence}

Even if they are often reported as solar-integrated values, apparent ${ }^{1} \mathrm{O}_{2}$ quantum yields do vary as a function of wavelength. Quantifying the spectral dependence of $\Phi_{\Delta}$ is particularly important for modeling of singlet oxygen steadystate concentrations in natural water bodies, as different wavelengths have different penetration depths in the water column. Thus, the use of monochromatic or solar-integrated quantum yields in environmental models results in erroneous $\left[{ }^{1} \mathrm{O}_{2}\right]_{\text {ss }}$ depth profiles. ${ }^{73}$
Apparent ${ }^{1} \mathrm{O}_{2}$ quantum yields were generally found to decrease as a function of wavelength (Figure 14). ${ }^{39,47,70,71,73,83}$
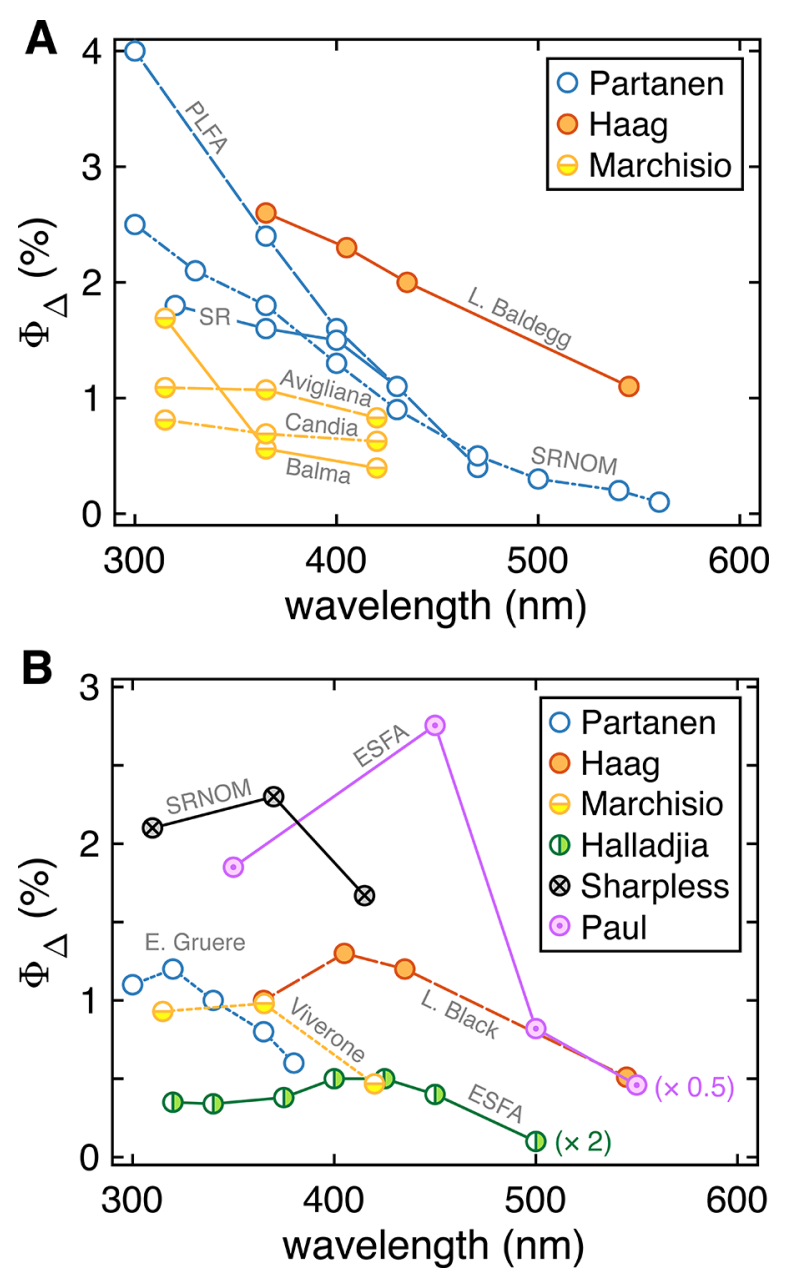

Figure 14. Wavelength dependence of $\Phi_{\Delta}$. In panel (A), we show waters displaying a monotonic decrease (linear or a bimodal linear) of apparent ${ }^{1} \mathrm{O}_{2}$ quantum yield as a function of wavelength, while in panel (B) are reported samples with a growth-decay trend. In panel (B), data from Paul et al. ${ }^{83}$ and Halladjia et al. ${ }^{70}$ are multiplied by 0.5 and 2, respectively, to aid visualizing spectral trends. Error bars are omitted for clarity. Legend: PLFA, Pony Lake fulvic acid; SRNOM, Suwannee River natural organic matter (IHSS isolate); SR, Suwannee River whole water sample; ESFA, Elliott Soil fulvic acid.

Several authors reported a relatively gentle decrease of $\Phi_{\Delta}$ with increasing wavelength in the UVB range, and a steeper decrease at wavelengths longer than $400-450 \mathrm{~nm}$, with values approaching zero for $\lambda>560 \mathrm{~nm} \cdot{ }^{39,47,71,83}$ This bimodal trend was observed, with some differences, for several DOM types including IHSS isolates such as SRFA and PLFA, and humic acids and organic matter samples isolated from freshwater bodies (Figure 14A). Two independent publications have reported a growth-decay trend in quantum yields vs wavelength with a maximum around $400-450 \mathrm{~nm}$ for fulvic acids extracted from soil samples (ESFA, Figure 14B). ${ }^{70,83}$ In an early work, Haag et al. ${ }^{47}$ measured the same growth-decay trend for Black lake humic acids (however, when considering the error bars, the trend might be more similar to the general bimodal one) and no wavelength-dependence for Fluka humic acids. Other authors also observed a growth-decay trend for other aquatic humic substances (Figure 14B)..$^{39,71,73}$ On the 
other hand, some DOM samples displayed an almost linear decrease in $\Phi_{\Delta}$ over relatively wide wavelength ranges (Figure 14A). ${ }^{39,47}$ To date, there is too little data to be able to make generalizations on the spectral dependence of $\Phi_{\Delta}$, also because studies often focused on different wavelength ranges, collected only a limited amount of experimental points (typically, $N=$ 3 ), and used lamps with very diverse spectral bandwidth. For example, Partanen et al. ${ }^{39}$ observed an almost linear decrease in PLFA $\Phi_{\Delta}$ between 300 and $450 \mathrm{~nm}$ ( $N=6$ wavelengths), while, for the same isolate, Sharpless ${ }^{71}$ obtained a "bimodal" decrease between 310 and $415 \mathrm{~nm}(N=3)$.

A common point is that ${ }^{1} \mathrm{O}_{2}$ quantum yields spectral dependence is significantly different from that of other photochemically produced reactive intermediates, ${ }^{73,237-240}$ or other DOM photodegradation products such as OCS, $\mathrm{CS}_{2}$, low-molecular-weight aldehydes, $\mathrm{CO}$, and $\mathrm{CO}_{2}{ }^{27,241-244}$ For these species, apparent quantum yields are typically characterized by steep exponential decrease as a function of wavelength (even if, in some cases, a leveling off above 380 $\mathrm{nm}$ is observed). ${ }^{241}$ Particularly remarkable is the difference between $\Phi_{\mathrm{T}}$ (measured via TMP oxidation) and $\Phi_{\Delta}$, as ${ }^{3} \mathrm{CDOM}^{*}$ is the precursor of ${ }^{1} \mathrm{O}_{2}$. Marchisio and coauthors ${ }^{73}$ showed that, for the same samples, $\Phi_{\mathrm{T}}$ decreases rapidly as a function of wavelength, while $\Phi_{\Delta}$ is still significant under visible blue light. The authors provided two possible explanations to justify these results. The first is that blue light excites only low-energy chromophores, which can be quenched by $\mathrm{O}_{2}$ but cannot react with TMP. In other words, $\Phi_{\mathrm{T}}$ measured via TMP loss is likely to provide only the wavelength-dependence of high-energy triplets, and not of the entire ${ }^{3} \mathrm{CDOM}^{*}$ pool. Another hypothesis is that $\Phi_{\Delta}$ quantified via FFA depletion detects two pools of ${ }^{1} \mathrm{O}_{2}$ (i.e., ${ }^{1} \mathrm{O}_{2}$ formed directly in the bulk phase by low-molecular-weight chromophores, and ${ }^{1} \mathrm{O}_{2}$ that diffuses from hydrophobic cores of high-molecular-weight sensitizers to the bulk), while TMP can only measure the triplets formed directly in the solution bulk. Under blue light, only high-molecular-weight compounds absorb light: under this condition, FFA can still detect the ${ }^{1} \mathrm{O}_{2}$ that diffuses out of these large sensitizers, resulting in a measurable ${ }^{1} \mathrm{O}_{2}$ quantum yield; on the other hand, TMP cannot react with these triplets, yielding an apparent $\Phi_{\mathrm{T}}=0$.

The fact that $\Phi_{\Delta}$ extends into the visible has important consequences for modeling ${ }^{1} \mathrm{O}_{2}$ steady-state concentration in water bodies, and therefore on the predictions of micropollutant's half-lives. Using their $\Phi_{\Delta}$ spectral dependence and a summer reference irradiance spectrum at $40^{\circ} \mathrm{N}$, Partanen and coauthors ${ }^{39}$ found that visible light (i.e., $>400 \mathrm{~nm}$ ) accounts for 25\% (PLFA) and 42\% (SRNOM) of the total near-surface daily ${ }^{1} \mathrm{O}_{2}$ production. This result indicates that ${ }^{1} \mathrm{O}_{2}$ production is still relevant in systems where UV light is attenuated, such as in lower depths of the water column.

Early authors found it "striking" that $\Phi_{\Delta}$ does change as a function of wavelength, as direct photolysis quantum yields of micropollutants are typically wavelength-independent. ${ }^{47}$ Nevertheless, they did acknowledge the complex nature of humic acids, and hypothesized that energy transfer between higher- and lower-energy excited states chromophores could explain the $\Phi_{\Delta}$ spectral dependence. ${ }^{47}$ More recently, Partanen and co-workers ${ }^{39}$ justified the wavelength-dependence in terms of the energy-gap rule. The energy-gap rule states that chromophores with small $S_{0}-S_{1}$ energy gap (i.e., having a red-shifted absorption; $S_{0}$ and $S_{1}$ indicates the ground state and the first singlet excited state, respectively) have a more favorable $S_{1} \rightarrow S_{0}$ internal conversion (i.e., radiationless decay) ${ }^{88}$ Internal conversion competes with fluorescence and intersystem crossing (Figure 2), resulting in low fluorescence and triplet quantum yields. Since $\Phi_{\Delta}$ is directly proportional to $\Phi_{\mathrm{T}}$ (eq 2), a decrease in triplet quantum yield will lead to a reduction in $\Phi_{\Delta}$. Indeed, reports of apparent triplet quantum yields as a function of wavelength confirmed the expected trend (see also above), even if the use of TMP might bias the results toward high-energy triplets. ${ }^{73}$ On the other hand, small $S_{0}-S_{1}$ energy gap will also lead to a small $S_{0}-T_{1}$ energy gap, as triplet excited states are lower in energy than the corresponding singlets. Thus, the $\mathrm{T}_{1} \rightarrow \mathrm{S}_{0}$ relaxation will be more efficient, resulting in larger $k_{\mathrm{d}}^{\mathrm{T}}$ values and therefore smaller triplet lifetimes. Since $k_{\mathrm{d}}^{\mathrm{T}}$ is inversely proportional to $\Phi_{\Delta}$ (eq 2), apparent ${ }^{1} \mathrm{O}_{2}$ quantum yields are expected to decrease. To date, only Sharpless ${ }^{71}$ collected wavelengthdependence data of triplet CDOM lifetimes, showing that, in the range $310-415 \mathrm{~nm}, \tau_{\mathrm{T}}$ is independent of excitation wavelength. Thus, in this wavelength interval, differences in $\Phi_{\Delta}$ among different wavelengths cannot be explained by differences in triplet lifetimes. A third possibility is that longwavelength chromophores have lower $\mathrm{O}_{2}$ quenching rates constants or produce ${ }^{1} \mathrm{O}_{2}$ less efficiently when quenched by $\mathrm{O}_{2}$ (i.e., have lower $f_{\Delta}$, the fraction of $\mathrm{O}_{2}$ quenching that leads to ${ }^{1} \mathrm{O}_{2}$ formation; see eq 2). There is not enough data yet to assess the viability of this third possibility.

The $\Phi_{\Delta}$ wavelength-dependence was also explained in the context of the charge transfer model. ${ }^{71}$ In this case, the low triplet (thus, singlet oxygen) quantum yields in the visible are the result of an increased proportion of charge transfer interactions. Involvement of CT complexes either prevents the formation of triplets or causes their deactivation, resulting in a low population of ${ }^{3} \mathrm{CDOM}^{*}$ states.

\subsection{Seasonal and Spatial Variability}

Some authors investigated the spatial and seasonal variability in apparent ${ }^{1} \mathrm{O}_{2}$ quantum yields in various watersheds, mostly located in the United States (Table 8). In all cases, ${ }^{1} \mathrm{O}_{2}$ dynamics appeared to be highly influenced by the particular characteristics of the watershed, including vegetation type and cover, climate, the extent of urbanization, and anthropogenic inputs, all factors that have direct effects on dissolved organic matter inputs. Below, we summarize relevant results that illustrate the correlations between geographical and ecosystem properties and DOM photoreactivity. In a few cases, the authors reported spatial ${ }^{34}$ or temporal ${ }^{83}$ trends but did not analyze in detail the possible causes for the variability and are therefore not included in this discussion. In these studies, DOM photodegradation was invoked as the main cause of seasonal trends, even if several lines of evidence indicated that other factors played a role (see section 5.3.2).

5.5.1. Freshwater to Saltwater Systems. In 2000, Sandvik and coauthors ${ }^{37}$ published the first study investigating temporal and spatial $\Phi_{\Delta}$ dynamics along salinity transects of the Mississippi and Atchafalaya river plumes inside the Gulf of Mexico. In this work, the authors reported almost no changes in $\Phi_{\Delta}$ from 0 to $30 \mathrm{ppt}$ of salinity for the Mississippi plume, and a slight (but not significant) increase for the Atchafalaya plume. In addition, no significant variations were detected between the June and the February cruise. As the authors point out, these results describe only the behavior of high-molecularweight fractions $(>3 \mathrm{kDa})$, as ${ }^{1} \mathrm{O}_{2}$ quantum yields were measured on ultrafiltrate isolates, and not on whole waters (see 
Table 8. Studies Investigating Seasonal and Spatial Variability in Apparent ${ }^{1} \mathrm{O}_{2}$ Quantum Yields

\begin{tabular}{|c|c|c|c|}
\hline reference & location & seasonal variability & spatial variability \\
\hline Sandvik et al., $2000^{37}$ & $\begin{array}{l}\text { Mississippi and Atchafalaya river } \\
\text { plumes, US }\end{array}$ & no changes ${ }^{a}$ & no changes ${ }^{a}$ \\
\hline Paul et al., $2004^{83}$ & Scandinavian lakes and streams & spring $>$ fall & \\
\hline Peterson et al., $2012^{36}$ & Lake Superior, US & spring $<$ summer & $\begin{array}{l}\text { harbor < offshore (spring); max } 5.6 \mathrm{~km} \text { from harbor } \\
\quad(\text { summer })\end{array}$ \\
\hline McCabe and Arnold, $2016^{34}$ & Prairie Pothole peatlands, US & spring $<$ summer $\leq$ fall & semipermanent $>$ temporary peatlands \\
\hline McKay et al., $2017^{35}$ & Florida Everglades, US & wet (Dec-May) < dry (Jun-Nov) & marsh $>$ estuarine \\
\hline Berg et al., $2019^{77}$ & St. Louis river, US & & headwater > lake estuary \\
\hline
\end{tabular}

section 4.3.1 for drawbacks of ultrafiltration). Significant changes in optical parameters (i.e., spectral slope) between whole waters and isolates confirmed that the proportion of low-molecular-weight material, which was lost during ultrafiltration, increased along the salinity transect.

McKay and coauthors ${ }^{35}$ investigated the spatial and temporal $\Phi_{\Delta}$ variations in water samples collected in the Everglades National Park in Florida, US. The Everglades are the largest subtropical wetlands in the US and are characterized by large variability in DOM type across subenvironments. ${ }^{245,246}$ Whole water samples were collected both in the dry (December to May) and the wet (June to November) season in several locations along the Shark River Slough, the Taylor Slough, and in the Florida Bay, which are close to the southern-most edge of the National Park. Overall, apparent ${ }^{1} \mathrm{O}_{2}$ quantum yields in the two sloughs increased from the wet to the dry season, which is consistent with changes in DOM dynamics in the estuaries. Indeed, during the dry season, there is an inflow of marine seagrass-derived DOM in the slough estuary, which is characterized by low aromaticity and molecular weight (thus, high $\Phi_{\Delta}$ ), while, in the wet season, slough DOM is dominated by terrestrial inputs. In addition, there was an overall decrease in apparent ${ }^{1} \mathrm{O}_{2}$ quantum yields from north to south (i.e., along a salinity transect toward the sea), which might be caused either by photobleaching or by changes in DOM inputs. The authors ruled out the photobleaching hypothesis, mostly because of the high level of shading due to aquatic vegetation and elevated CDOM content. We also note that photobleaching has been found to increase $\Phi_{\Delta}{ }^{42}$ which would give the opposite of what was observed. Therefore, changes in DOM sources appeared as the most likely explanation and were confirmed by bulk optical measurements. Freshwater marshes (present in the North) are characterized by soil (peat and marl) and aquatic vegetation (periphyton) inputs, with overall high molecular weight and intermediate aromaticity. On the other hand, Southern environments are characterized by mangrove forests, which release high-molecular-weight and high-aromaticity DOM. A large part of mangrove leachates consists of tannins, ${ }^{247}$ which are good antioxidants but poor ${ }^{1} \mathrm{O}_{2}$ sensitizers. The author speculated that the lower $\Phi_{\Delta}$ values in the southern sites are the result of a decreased amount of ${ }^{3} \mathrm{CDOM}^{*}$ precursors, implying that mangroves are the main photoreactivity driver in the southern sites.

5.5.2. Lake Superior. Peterson et al. ${ }^{36}$ performed the first multiyear study of apparent ${ }^{1} \mathrm{O}_{2}$ dynamics in whole water samples collected in Lake Superior, the largest freshwater lake on Earth. The authors collected samples in selected tributaries, including the St. Louis river that feeds into the DuluthSuperior Harbor, and $5 \mathrm{~m}$-depth lake samples in several locations offshore. The sampling campaign was conducted in late spring (May-June) and late summer (August-September) between 2007 and 2009. Overall, samples collected in the same season in different years showed a remarkably reproducible behavior, with apparent ${ }^{1} \mathrm{O}_{2}$ quantum yields being overall higher in the summer months (late spring: $0.93-3.3 \%$; late summer: $2.4-5.5 \%)$. Within each season, $\Phi_{\Delta}$ showed very diverse spatial trends: in spring, apparent ${ }^{1} \mathrm{O}_{2}$ quantum yields increased monotonically from river to off-shore, while, in summer, $\Phi_{\Delta}$ showed a pronounced growth-decay trend, with a maximum at $5.6 \mathrm{~km}$ offshore of the harbor. The authors speculated that the summer "spike" must be related to changes in DOM composition, possibly because of in situ production of microbial DOM, photodegradation of riverine DOM, anthropogenic inputs, or a combination of these factors. On the other hand, in both seasons, singlet oxygen production rates showed a well-behaved linear decrease as a function of distance from the harbor, indicating that, overall, changes in [DOC] were more important than variations in $\Phi_{\Delta}$ in defining ${ }^{1} \mathrm{O}_{2}$ production rates.

A few years later, Berg et al. ${ }^{77}$ investigated the spatial $\Phi_{\Delta}$ dynamics along the St. Louis river, which is the second largest tributary of Lake Superior. This river was selected because of its highly diverse watershed, which is characterized by abundant terrestrial DOM inputs from forests and wetlands in the riverhead, and by a larger proportion of urban-derived inputs as the river flows toward the lake. Grab samples were collected in August 2016 in eight locations along the river and the lake. Bulk optical and mass spectroscopy measurements confirmed that DOM becomes smaller and less aromatic as it travels downstream as a result of environmental processing and/or microbial input. Consistently, $f_{\text {TMP }}$ and $\Phi_{\Delta}$ increased moving from the upper watershed toward Lake Superior, with a spike at a wastewater treatment plan effluent discharge point.

\subsection{Short Summary and Research Gaps}

Among bulk water chemistry parameters, dissolved oxygen and inorganic salts are two factors that can potentially exert a considerable influence on apparent ${ }^{1} \mathrm{O}_{2}$ quantum yields in environmentally relevant conditions but have not been thoroughly investigated. There is a solid mechanistic justification for the observed changes in $\Phi_{\Delta}$ as a function of $\left[\mathrm{O}_{2}\right]$, which is particularly evident at $\left[\mathrm{O}_{2}\right]<200-250 \mu \mathrm{mol}$ $\mathrm{L}^{-1}$ (Figure 13A). Dissolved oxygen levels below this threshold are not unrealistic and might result from a series of factors including increasing temperature, increasing DOC content, and changes in the trophic status of the water body. ${ }^{96,217,219,220}$ Also, oxygen dependence makes apparent ${ }^{1} \mathrm{O}_{2}$ quantum yields temperature dependent, albeit indirectly, which goes against the commonly held view that photochemical reactions are almost insensitive to temperature. A notable consequence is 
that quantum yield measurements performed under standardized laboratory conditions might not be well suited to reproduce quantum yields in the natural environment. Notably, there have not yet been apparent ${ }^{1} \mathrm{O}_{2}$ quantum yield measurements performed outside of the laboratory. Comparison of laboratory measurements to those performed outdoor (i.e., under realistic environmental conditions in terms of temperature and $\mathrm{O}_{2}$ levels) will help clarifying this issue.

The effect of salt content on $\Phi_{\Delta}$, which is crucial to improve our understanding of ${ }^{1} \mathrm{O}_{2}$ photochemistry in marine environments, is still mostly unexplored. Glover and Rosario-Ortiz ${ }^{54}$ observed an increase in $\Phi_{\Delta}$ with increasing salt content, likely due to increased triplet lifetimes; however, more investigations are needed to reconcile some of the discrepancies in the photochemical reactivity of ${ }^{1} \mathrm{O}_{2}$ and triplet organic matter in the presence of halides.

A recent study ${ }^{79}$ found that metals decrease $\Phi_{\Delta}$ values proportionally to their binding ability with dissolved organic matter due to an increased proportion of static and dynamic quenching. To date, the role of metals in environmental samples has not yet been investigated. This aspect might be relevant to explain variability in photoreactivity in environments experiencing metal pollution or metal leaching from natural sources.

Changes in average molecular weight explain most of the reported trends in apparent ${ }^{1} \mathrm{O}_{2}$ quantum yields as a function of optical and molecular features, as well as the results of DOM fractionation experiments and (photo)chemical oxidation. The general understanding is that low-molecular-weight fractions have higher $\Phi_{\Delta}$ than high-molecular-weight fractions. This result is often justified in terms of a charge transfer model, ${ }^{28,214}$ even if other explanations have also been proposed. There is also evidence in support of the role of redox-active moieties (i.e., phenols and quinones) and aromatic ketones in the photochemical production of ${ }^{1} \mathrm{O}_{2}$ from CDOM. Furthermore, correlations between apparent ${ }^{1} \mathrm{O}_{2}$ and triplet quantum yields (measured via TMP oxidation) corroborate the idea that ${ }^{3} \mathrm{CDOM}^{*}$ is a precursor of ${ }^{1} \mathrm{O}_{2}$, and that there is a substantial overlap between the ${ }^{3} \mathrm{CDOM}^{*}$ pool that oxidizes TMP and the one that produces ${ }^{1} \mathrm{O}_{2}$. Several authors have proposed the use of easily measured spectral features such as E2:E3 or $S_{300-600}$ as predictors of apparent ${ }^{1} \mathrm{O}_{2}$ quantum yields. However, the overall lack of reproducibility in slopes and intercepts of the linear regression models (likely resulting from systematic errors in measuring $\Phi_{\Delta}$ ) does not yet allow a confident use of these spectroscopic parameters as quantum yield predictors. Improvements in measurement protocols (see section 3.4) should also address this standing challenge.

Several authors used reductive treatments to confirm the involvement of aromatic ketones in ${ }^{1} \mathrm{O}_{2}$ production and verify the validity of the charge transfer model. However, difficulties in interpreting the results of these experiments raises the question of whether $\mathrm{NaBH}_{4}$ reduction is a meaningful experiment to test such hypotheses. Quantum yields depend not only on the ${ }^{1} \mathrm{O}_{2}$ production rate but also on the rate of light absorption, which are two parameters that can be influenced to a different extent by the reduction process. In addition, if aromatic ketones were the only photosensitizer present, a decrease in their concentration will cause a decrease in both ${ }^{1} \mathrm{O}_{2}$ production and light absorption rates, which should result in no net changes in $\Phi_{\Delta}$, not in its decrease. Furthermore, synthetic chemistry protocols for $\mathrm{NaBH}_{4}$ reduction reactions in water typically require an extraction step with dichloromethane or diethyl ether to isolate the alcohol from the boron salts; ${ }^{248-251}$ also general protocols in organic solvents require acidification and extraction. ${ }^{252}$ In 2016, Schendorf et al. ${ }^{253}$ developed a standard protocol for $\mathrm{NaBH}_{4}$ reduction of humic substances that also involves a purification step (i.e., size-exclusion chromatography) to remove the borate salts. This protocol does represent an advancement compared to previous procedures, but still does not ensure that all hydroxyl-borate complexes within DOM are hydrolyzed. Schendorf et al. ${ }^{253}$ reported minimal changes in optical properties before and after chromatographic purification, suggesting that borate salts have negligible effects on DOM absorption. However, previous research showed that caffeic acid-borate complexes have a similar absorption spectrum but a different reactivity compared to the uncomplexed molecule. ${ }^{254}$ In future studies, control experiments should confirm that the presence of boron complexes with phenols and other alcoholic groups does not influence the interpretation of the photochemical results. On the other hand, oxidative treatments overall cause an increase in $\Phi_{\Delta}$. Despite being generally interpreted as due to a decrease in molecular weight, results for other PPRIs and other spectroscopic parameters suggest that, in addition to molecular weight, other factors might decrease apparent ${ }^{1} \mathrm{O}_{2}$ quantum yields. In addition, it is still unclear if and to what extent environmentally relevant oxidative conditions (i.e., photooxidation or reactivity with ozone) might influence spatial and temporal variability of $\Phi_{\Delta}$ in natural samples

Assessing the wavelength dependence of apparent quantum yields is crucial for modeling steady-state concentrations in water bodies. This fact is especially relevant for ${ }^{1} \mathrm{O}_{2}$ as, different from other PPRIs, its production is still quantitatively important with visible blue light. Thus, the use of solarintegrated $\Phi_{\Delta}$ values for modeling purposes might not be appropriate. More studies should focus on determining the spectral dependence of this parameter.

\section{FINAL REMARKS}

The study of photochemistry in environmental systems has grown tremendously over the past decades, and along with it the study of ${ }^{1} \mathrm{O}_{2}$ in environmental waters. In particular, interest in measuring apparent singlet oxygen quantum yields of a wide range of environmental samples has steadily increased over the past years. In the first part of this review, we summarized the main challenges related to the measurement of this parameter, and we provided practical recommendations to improve current measurement protocols (see section 3.4). Although some experimental details still need to be optimized, we believe that the current guidelines will help eliminate a good fraction of the interstudy variability that has so far hindered our understanding of ${ }^{1} \mathrm{O}_{2}$ production in natural systems. These technical advancements will improve the possibility of confidently aggregating results from independent studies, and this, in turn, will help to answer many of the still open questions pertaining to ${ }^{1} \mathrm{O}_{2}$ in the natural environment-many of which were reviewed and summarized in the second part of this review (see sections 4.4 and 5.6). We anticipate that an improved understanding of ${ }^{1} \mathrm{O}_{2}$ production and variability in natural systems will lead to more accurate predictive models for micropollutants' fate and biogeochemical cycles.

To conclude, we want to stress that many of the technical issues highlighted in this review are not specific to singlet oxygen. Specifically, all of the points raised in section 3.2.1 
(Measuring light absorption) are directly valid for quantum yield measurements of any photochemically produced reactive species (i.e., ${ }^{\bullet} \mathrm{OH},{ }^{3} \mathrm{CDOM}^{*}, \mathrm{O}_{2}^{\bullet}$, etc.) or photodegradation product (i.e., $\mathrm{CO}_{2}, \mathrm{CO}$, ammonia, etc.) of natural organic matter samples or complex organic mixtures. For example, $\mathrm{Hu}$ et al. $^{144}$ point out several technical problems in dissolved inorganic carbon (i.e., $\mathrm{CO}_{2}$ ) photoproduction quantum yield measurements that closely match what we describe here for ${ }^{1} \mathrm{O}_{2}$. We have hope that our work will benefit many researchers in the broad environmental chemistry community and beyond.

\section{ASSOCIATED CONTENT}

\section{SI Supporting Information}

The Supporting Information is available free of charge at https://pubs.acs.org/doi/10.1021/acs.chemrev.0c00781.

Estimation of self-quenching of ${ }^{1} \mathrm{O}_{2}$ by DOM and chemical probes; $R_{\mathrm{abs}}$ equations: variants and approximations; errors induced by the choice of the upper integration wavelength $\left(\lambda_{\text {up }}\right)$; derivation of actinometry equations; estimation of errors in apparent ${ }^{1} \mathrm{O}_{2}$ quantum yields; supplementary tables with technical data from papers reporting apparent ${ }^{1} \mathrm{O}_{2}$ quantum yield values of natural samples (PDF)

Singlet oxygen quantum yields database (XLSX)

\section{AUTHOR INFORMATION}

\section{Corresponding Author}

Kristopher McNeill - Institute of Biogeochemistry and Pollutant Dynamics (IBP), Department of Environmental Systems Science, ETH Zürich, 8092 Zürich, Switzerland; ๑ orcid.org/0000-0002-2981-2227;

Email: kristopher.mcneill@env.ethz.ch

\section{Authors}

Rachele Ossola - Institute of Biogeochemistry and Pollutant Dynamics (IBP), Department of Environmental Systems Science, ETH Zürich, 8092 Zürich, Switzerland; ○ orcid.org/0000-0003-4648-5958

Oskar Martin Jönsson - Institute of Biogeochemistry and Pollutant Dynamics (IBP), Department of Environmental Systems Science, ETH Zürich, 8092 Zürich, Switzerland

Kyle Moor - Utah Water Research Laboratory, Department of Civil and Environmental Engineering, Utah State University, 84322 Logan, Utah, United States; 10 orcid.org/ 0000-0002-3152-0354

Complete contact information is available at: https://pubs.acs.org/10.1021/acs.chemrev.0c00781

\section{Notes}

The authors declare no competing financial interest. Nine additional papers reporting ${ }^{1} \mathrm{O}_{2}$ quantum yield values (Wang et al., ${ }^{255}$ Palma et al., ${ }^{256}$ Chen et al., ${ }^{257}$ Wasswa et al., ${ }^{258}$ Wan et al., ${ }^{259}$ Guo et al., ${ }^{260}$ Leresche et al., ${ }^{261}$ Wang et al., ${ }^{262}$ and Zhou et al. ${ }^{263}$ ) have been published after March 2020 and are not included in this review.

\section{Biographies}

Rachele Ossola received her undergraduate degree in Chemistry from the University of Milan, Italy (2014) and her Master's degree in Molecular and Biological Chemistry from EPFL, Switzerland (2016). During her Master's program, she conducted research on ultrafast spectroscopy of perovskites for dye-sensitized solar cells and on solar disinfection of human viruses under the supervision of Prof. JacquesE. Moser and Prof. Tamar Kohn. Since 2016, she is a Ph.D. student with Prof. Kristopher McNeill at ETH Zurich, where she works on the photochemistry and photodegradation mechanisms of dissolved organic matter.

Oskar M. Jönsson received his Bachelor's (2017) and Master's (2020) degrees in Environmental Sciences from ETH Zurich, Switzerland. During his bachelor thesis, he worked on photochemically produced reactive intermediates in northern peat bogs under the supervision of Dr. Michael Sander and Prof. Kristopher McNeill. In the following year, he worked in the McNeill group as a student assistant. During his Master's degree, he left the chemistry sphere to work on sustainability in international trade. He is now an Advisor at the State Secretariat for Economic Affairs in Switzerland.

Kyle Moor received his B.S. in chemistry from Virginia Polytechnic Institute and State University (2010) and his Ph.D. in environmental engineering from Yale University (2016) with Prof. Jaehong Kim. Following his Ph.D., he was awarded an ETH Postdoctoral Fellowship and joined the Environmental Chemistry group at ETH Zurich with Prof. McNeill, where he developed laser spectroscopy techniques (i.e., time-resolved ${ }^{1} \mathrm{O}_{2}$ phosphorescence) to study the photochemistry of dissolved organic matter. He is currently an assistant professor at Utah State University in the Department of Civil and Environmental Engineering. His research at the Utah Water Research Laboratory focuses on understanding pollutant degradation in aquatic systems and elucidating reaction pathways with laser spectroscopy.

Kristopher McNeill received his B.A. in chemistry (1992) from Reed College and his Ph.D. in chemistry (1997) from the University of California, Berkeley, under Profs. Robert Bergman and Richard Andersen. Following a postdoctoral research position (1997-1999) with Prof. Philip Gschwend at MIT, he started his independent career in the Department of Chemistry at the University of Minnesota (2000-2009). He took up his current position as Professor of Environmental Chemistry at ETH Zurich in 2009. His research group studies reaction mechanisms involved in the transformation of organic molecules in aquatic environments. His group has had a strong focus on photochemical transformations, including on the role of singlet oxygen in the fate of contaminants, viruses, and naturally occurring organic compounds in surface waters.

\section{ACKNOWLEDGMENTS}

We thank Gordon Getzinger and Baptiste Clerc for helpful discussions and Nadine Borduas-Dedekind and Sebastian Zala for their contributions on sections 4.2.5 and 4.3.2. Oskar M. Jönsson gratefully acknowledges funding from the Master Scholarship Programme by the Department of Environmental Systems Science at ETH Zürich. We also thank Bill Arnold, Laleen Bodhipaksha, Frank Leresche, Meghan O'Connor, Xiaolei Qu, Fernando Rosario-Ortiz, Charlie Sharpless, Weihua Song, Davide Vione, Jannis Wenk, and Huaxi Zhou for providing us access to their numerical data. Last, we acknowledge the anonymous reviewers for their insightful comments and suggestions on the manuscript. We gratefully acknowledge funding from ETH Zurich (Master Scholarship Programme, USYS) and Swiss National Science Foundation (SNSF Grant No. 200020_188565).

\section{ABBREVIATIONS LIST}

$A$ absorbance

$A_{\lambda}$ absorbance at wavelength $\lambda$ 
(C)DOM (chromophoric) dissolved organic matter

${ }^{3} \mathrm{CDOM}^{*}$ triplet excited state of chromophoric dissolved organic matter

CT charge transfer

2,5-DMF 2,5-dimethylfuran

DBC dissolved black carbon

$E_{\mathrm{SHE}}^{0 *}$ excited state reduction potential vs standard hydrogen electrode

E2:E3 ratio of the absorbance at 250 or $254 \mathrm{~nm}$ to that at $365 \mathrm{~nm}$

EfOM effluent organic matter

EOM extracellular organic matter

EDC electron donating capacity

EAC electron accepting capacity

FFA furfuryl alcohol

FWHM full width at half-maximum

FA fulvic acid

$f_{\Delta}$ fraction of triplet physical quenching that leads to singlet oxygen production

$f_{\mathrm{P}}$ quantum yield coefficient measured with probe $\mathrm{P}$

HA humic acid

HPOA hydrophobic organic acid fraction

HPON hydrophobic organic neutral fraction

HPI hydrophilic fraction

IC internal conversion

ISC intersystem crossing

$I_{0, \lambda}$ absolute spectral irradiance (at the liquid surface)

$I_{\lambda, \text { rel }}$ relative spectral irradiance

$I_{\lambda, \mathrm{m}}$ measured spectral irradiance

IHSS International Humic Substances Society

$k_{\mathrm{d}}^{\mathrm{T}}$ (natural) triplet decay rate constant

$k_{\mathrm{d}}^{\Delta}$ (natural) singlet oxygen decay rate constant

$k_{\mathrm{O} 2}$ bimolecular rate constants for the physical quenching of excited triplet states with $\mathrm{O}_{2}$

$k_{\mathrm{rxn}, 1 \mathrm{O} 2}^{\mathrm{A}}$ bimolecular rate constant for the chemical reaction of A with singlet oxygen

$k_{\mathrm{rxn}, \bullet \mathrm{OH}}^{\mathrm{A}}$ bimolecular rate constant for the chemical reaction of A with hydroxyl radicals

$k_{\text {phys, } 1 \mathrm{O} 2}^{\mathrm{A}}$ bimolecular rate constant for the physical quenching of A with singlet oxygen

$k_{\text {obs,A }}$ pseudo-first-order rate constant of A

$k_{\mathrm{obs}, \mathrm{A}}^{\mathrm{Sens}}$ pseudo-first-order rate constant of A measured in the presence of a sensitizer Sens

$k_{\mathrm{r}}^{\Delta}$ radiative relaxation rate constant of singlet oxygen to ground state oxygen

$l$ optical path length

$N$ number of samples

2-NBA 2-nitrobenzaldehyde

NOM natural organic matter

${ }^{1} \mathrm{O}_{2}$ singlet oxygen

$\left[{ }^{1} \mathrm{O}_{2}\right]_{\text {ss }}$ singlet oxygen steady-state concentration

OD optical density

- OH hydroxyl radicals

$\left[{ }^{\bullet} \mathrm{OH}\right]_{\text {ss }}$ hydroxyl radicals steady-state concentration

OM organic matter

$\mathrm{P}$ generic singlet oxygen probe

PPRI photochemically produced reactive intermediate

PNA $p$-nitroanisole

py pyridine

PNAP $p$-nitroacetophenone

PN perinaphthenone

PLFA Pony Lake fulvic acid

PM particulate matter
$R_{\mathrm{abs}}$ rate of light absorption

$R_{\mathrm{abs}}^{i}$ rate of light absorption of component $i$

$R_{\mathrm{abs}}^{0}$ initial rate of light absorption

$R_{1 \mathrm{O} 2}$ singlet oxygen formation rate

$R_{1 \mathrm{O} 2}^{0}$ initial singlet oxygen formation rate

$R_{\mathrm{P}}$ probe (P) degradation rate

$R_{\mathrm{P}}^{0}$ initial probe degradation rate

$\mathrm{RB}$ Rose Bengal

$\mathrm{RO}$ reverse osmosis

$S$ generic singlet oxygen signal

$S_{\lambda}^{\text {sol }}$ solution screening factor at wavelength $\lambda$

SRFA Suwannee River fulvic acid

SRHA Suwannee River humic acid

SRNOM Suwannee River natural organic matter

$\mathrm{SUVA}_{254}$ specific ultraviolet absorbance at $254 \mathrm{~nm}$

SOM soil organic matter

SOA secondary organic aerosol

SPE solid-phase extraction

$S_{300-600}$ spectral slope over the $300-600 \mathrm{~nm}$ wavelength

range

$S_{\mathrm{R}}$ spectral slope ratio

TMPyP meso-tetra( $N$-methyl-4-pyridyl)porphyrin

TOC total organic carbon

TPIA transphilic organic acid fraction

TPIN transphilic organic neutral fraction

TMP 2,4,6-trimethylphenol (triplet probe)

WE water-extractable soil organic matter

$\alpha_{\lambda}^{\mathrm{A}}$ decadic absorption coefficient of $\mathrm{A}$

$\varepsilon_{\lambda}^{\mathrm{A}}$ decadic molar extinction coefficient of $\mathrm{A}$

$\gamma$ scaling factor needed to converted relative into absolute spectral irradiance

$\Phi_{\Delta}$ or $\Phi_{\Delta}^{\text {Sens }}$ (apparent, if referred to DOM) singlet oxygen quantum yield of a sensitizer Sens

$\lambda_{\text {up }}$ upper integration wavelength (in rate of light absorption calculations)

$\Phi_{\mathrm{T}}$ (apparent, if referred to DOM) triplet quantum yield

$\Phi_{\mathrm{F}}$ fluorescence quantum yield

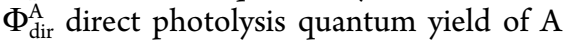

$\tau_{\Delta}$ (natural) singlet oxygen lifetime

$\tau_{\mathrm{T}}$ (natural) triplet excited state lifetime

\section{REFERENCES}

(1) Latch, D. E. The Role of Singlet Oxygen in Surface Water Photochemistry. In Surface Water Photochemistry; The Royal Chemical Society, 2015; pp 139-165.

(2) Schwarzenbach, R. P.; Gschwend, P. M.; Imboden, D. M. Indirect Photolysis: Reactions with Photooxidants in Natural Waters and in the Atmosphere. In Environmental Organic Chemistry; John Wiley \& Sons, Inc., 2002; pp 655-686.

(3) Zepp, R. G.; Wolfe, N. L.; Baughman, G. L.; Hollis, R. C. Singlet Oxygen in Natural Waters. Nature 1977, 267, 421-423.

(4) Larson, R. A.; Marley, K. A. Singlet Oxygen in the Environment. In Environmental Photochemistry; Springer: Berlin, Heidelberg, 1999; pp 123-137.

(5) McNeill, K.; Canonica, S. Triplet State Dissolved Organic Matter in Aquatic Photochemistry: Reaction Mechanisms, Substrate Scope, and Photophysical Properties. Environ. Sci. Process. Impacts 2016, 18, 1381-1399.

(6) Faust, B. C.; Allen, J. M. Aqueous-Phase Photochemical Sources of Peroxyl Radicals and Singlet Molecular Oxygen in Clouds and Fog. J. Geophys. Res. 1992, 97, 12913-12926.

(7) Kaur, R.; Labins, J. R.; Helbock, S. S.; Jiang, W.; Bein, K. J.; Zhang, Q.; Anastasio, C. Photooxidants from Brown Carbon and Other Chromophores in Illuminated Particle Extracts. Atmos. Chem. Phys. 2019, 19, 6579-6594. 
(8) Kaur, R.; Anastasio, C. Light Absorption and the Photoformation of Hydroxyl Radical and Singlet Oxygen in Fog Waters. Atmos. Environ. 2017, 164, 387-397.

(9) Manfrin, A.; Nizkorodov, S. A.; Malecha, K. T.; Getzinger, G. J.; McNeill, K.; Borduas-Dedekind, N. Reactive Oxygen Species Production from Secondary Organic Aerosols: The Importance of Singlet Oxygen. Environ. Sci. Technol. 2019, 53, 8553-8562.

(10) Cote, C. D.; Schneider, S. R.; Lyu, M.; Gao, S.; Gan, L.; Holod, A. J.; Chou, T. H. H.; Styler, S. A. Photochemical Production of Singlet Oxygen by Urban Road Dust. Environ. Sci. Technol. Lett. 2018, 5, 92-97.

(11) Bower, J. P.; Anastasio, C. Degradation of Organic Pollutants in/on Snow and Ice by Singlet Molecular Oxygen $\left({ }^{1} \mathrm{O}_{2} *\right)$ and an Organic Triplet Excited State. Environ. Sci. Process. Impacts 2014, 16, $748-756$.

(12) Remucal, C. K. The Role of Indirect Photochemical Degradation in the Environmental Fate of Pesticides: A Review. Environ. Sci. Process. Impacts 2014, 16, 628-653.

(13) Lundeen, R. A.; Janssen, E. M.-L.; Chu, C.; McNeill, K. Environmental Photochemistry of Amino Acids, Peptides and Proteins. Chimia 2014, 68, 812-817.

(14) Cadet, J.; Douki, T.; Ravanat, J.-L.; Mascio, P. D. Reactions of Singlet Oxygen with Nucleic Acids. In Singlet Oxygen: Applications in Biosciences and Nanosciences; The Royal Chemical Society, 2016; Vol. 1 , pp 393-407.

(15) García, N. A. New Trends in Photobiology: Singlet-MolecularOxygen-Mediated Photodegradation of Aquatic Phenolic Pollutants. A Kinetic and Mechanistic Overview. J. Photochem. Photobiol., B 1994, 22, 185-196.

(16) Di Mascio, P.; Martinez, G. R.; Miyamoto, S.; Ronsein, G. E.; Medeiros, M. H. G.; Cadet, J. Singlet Molecular Oxygen Reactions with Nucleic Acids, Lipids, and Proteins. Chem. Rev. 2019, 119, 2043-2086

(17) Nelson, K. L.; Boehm, A. B.; Davies-Colley, R. J.; Dodd, M. C.; Kohn, T.; Linden, K. G.; Liu, Y.; Maraccini, P. A.; McNeill, K.; Mitch, W. A.; et al. Sunlight-Mediated Inactivation of Health-Relevant Microorganisms in Water: A Review of Mechanisms and Modeling Approaches. Environ. Sci. Process. Impacts 2018, 20, 1089-1122.

(18) Kohn, T.; Nelson, K. L. Sunlight-Mediated Inactivation of MS2 Coliphage via Exogenous Singlet Oxygen Produced by Sensitizers in Natural Waters. Environ. Sci. Technol. 2007, 41, 192-197.

(19) Kohn, T.; Grandbois, M.; McNeill, K.; Nelson, K. L. Association with Natural Organic Matter Enhances the SunlightMediated Inactivation of MS2 Coliphage by Singlet Oxygen. Environ. Sci. Technol. 2007, 41, 4626-4632.

(20) Mattle, M. J.; Vione, D.; Kohn, T. Conceptual Model and Experimental Framework to Determine the Contributions of Direct and Indirect Photoreactions to the Solar Disinfection of MS2, PhiX174, and Adenovirus. Environ. Sci. Technol. 2015, 49, 334-342.

(21) Rosado-Lausell, S. L.; Wang, H.; Gutiérrez, L.; RomeroMaraccini, O. C.; Niu, X.-Z.; Gin, K. Y. H.; Croué, J.-P.; Nguyen, T. H. Roles of Singlet Oxygen and Triplet Excited State of Dissolved Organic Matter Formed by Different Organic Matters in Bacteriophage MS2 Inactivation. Water Res. 2013, 47, 4869-4879.

(22) Richards-Henderson, N. K.; Pham, A. T.; Kirk, B. B.; Anastasio, C. Secondary Organic Aerosol from Aqueous Reactions of Green Leaf Volatiles with Organic Triplet Excited States and Singlet Molecular Oxygen. Environ. Sci. Technol. 2015, 49, 268-276.

(23) George, C.; Ammann, M.; D’Anna, B.; Donaldson, D. J.; Nizkorodov, S. A. Heterogeneous Photochemistry in the Atmosphere. Chem. Rev. 2015, 115, 4218-4258.

(24) Cory, R. M.; McNeill, K.; Cotner, J. B.; Amado, A.; Purcell, J. M.; Marshall, A. G. Singlet Oxygen in the Coupled Photochemical and Biochemical Oxidation of Dissolved Organic Matter. Environ. Sci. Technol. 2010, 44, 3683-3689.

(25) Ward, C. P.; Cory, R. M. Assessing the Prevalence, Products, and Pathways of Dissolved Organic Matter Partial Photo-Oxidation in Arctic Surface Waters. Environ. Sci. Process. Impacts 2020, 22, 12141223.
(26) Waggoner, D. C.; Wozniak, A. S.; Cory, R. M.; Hatcher, P. G. The Role of Reactive Oxygen Species in the Degradation of Lignin Derived Dissolved Organic Matter. Geochim. Cosmochim. Acta 2017, 208, 171-184.

(27) Mopper, K.; Kieber, D. J.; Stubbins, A. Marine Photochemistry of Organic Matter: Processes and Impacts. In Biogeochemistry of Marine Dissolved Organic Matter, 2nd ed.; Academic Press: Boston, 2015; pp 389-450.

(28) McKay, G. Emerging Investigator Series: Critical Review of Photophysical Models for the Optical and Photochemical Properties of Dissolved Organic Matter. Environ. Sci. Process. Impacts 2020, 22, 1139-1165.

(29) Bresolí-Obach, R.; Torra, J.; Zanocco, R. P.; Zanocco, A. L.; Nonell, S. Singlet Oxygen Quantum Yield Determination Using Chemical Acceptors. In Reactive Oxygen Species: Methods and Protocols; Springer: New York, US, 2021; pp 165-188.

(30) Bodrato, M.; Vione, D. APEX (Aqueous Photochemistry of Environmentally Occurring Xenobiotics): A Free Software Tool to Predict the Kinetics of Photochemical Processes in Surface Waters. Environ. Sci. Process. Impacts 2014, 16, 732-740.

(31) Zhou, C.; Chen, J.; Xie, H.; Zhang, Y.; Li, Y.; Wang, Y.; Xie, Q.; Zhang, S. Modeling Photodegradation Kinetics of Organic Micropollutants in Water Bodies: A Case of the Yellow River Estuary. J. Hazard. Mater. 2018, 349, 60-67.

(32) Du, Z.; He, Y.; Fan, J.; Fu, H.; Zheng, S.; Xu, Z.; Qu, X.; Kong, A.; Zhu, D. Predicting Apparent Singlet Oxygen Quantum Yields of Dissolved Black Carbon and Humic Substances Using Spectroscopic Indices. Chemosphere 2018, 194, 405-413.

(33) Frimmel, F. H.; Bauer, H.; Putzien, J.; Murasecco, P.; Braun, A. M. Laser Flash Photolysis of Dissolved Aquatic Humic Material and the Sensitized Production of Singlet Oxygen. Environ. Sci. Technol. 1987, 21, 541-545.

(34) McCabe, A. J.; Arnold, W. A. Seasonal and Spatial Variabilities in the Water Chemistry of Prairie Pothole Wetlands Influence the Photoproduction of Reactive Intermediates. Chemosphere 2016, 155, 640-647.

(35) McKay, G.; Huang, W.; Romera-Castillo, C.; Crouch, J. E.; Rosario-Ortiz, F. L.; Jaffé, R. Predicting Reactive Intermediate Quantum Yields from Dissolved Organic Matter Photolysis Using Optical Properties and Antioxidant Capacity. Environ. Sci. Technol. 2017, 51, 5404-5413.

(36) Peterson, B. M.; McNally, A. M.; Cory, R. M.; Thoemke, J. D.; Cotner, J. B.; McNeill, K. Spatial and Temporal Distribution of Singlet Oxygen in Lake Superior. Environ. Sci. Technol. 2012, 46, $7222-7229$

(37) Sandvik, S. L. H.; Bilski, P.; Pakulski, J. D.; Chignell, C. F.; Coffin, R. B. Photogeneration of Singlet Oxygen and Free Radicals in Dissolved Organic Matter Isolated from the Mississippi and Atchafalaya River Plumes. Mar. Chem. 2000, 69, 139-152.

(38) De Laurentiis, E.; Buoso, S.; Maurino, V.; Minero, C.; Vione, D. Optical and Photochemical Characterization of Chromophoric Dissolved Organic Matter from Lakes in Terra Nova Bay, Antarctica. Evidence of Considerable Photoreactivity in an Extreme Environment. Environ. Sci. Technol. 2013, 47, 14089-14098.

(39) Partanen, S. B.; Erickson, P. R.; Latch, D. E.; Moor, K. J.; McNeill, K. Dissolved Organic Matter Singlet Oxygen Quantum Yields: Evaluation Using Time-Resolved Singlet Oxygen Phosphorescence. Environ. Sci. Technol. 2020, 54, 3316-3324.

(40) Bodhipaksha, L. C.; Sharpless, C. M.; Chin, Y.-P.; Sander, M.; Langston, W. K.; MacKay, A. A. Triplet Photochemistry of Effluent and Natural Organic Matter in Whole Water and Isolates from Effluent-Receiving Rivers. Environ. Sci. Technol. 2015, 49, 3453-3463.

(41) Bodhipaksha, L. C.; Sharpless, C. M.; Chin, Y.-P.; MacKay, A. A. Role of Effluent Organic Matter in the Photochemical Degradation of Compounds of Wastewater Origin. Water Res. 2017, 110, 170179.

(42) Sharpless, C. M.; Aeschbacher, M.; Page, S. E.; Wenk, J.; Sander, M.; McNeill, K. Photooxidation-Induced Changes in Optical, 
Electrochemical, and Photochemical Properties of Humic Substances. Environ. Sci. Technol. 2014, 48, 2688-2696.

(43) Leresche, F.; McKay, G.; Kurtz, T.; von Gunten, U.; Canonica, S.; Rosario-Ortiz, F. L. Effects of Ozone on the Photochemical and Photophysical Properties of Dissolved Organic Matter. Environ. Sci. Technol. 2019, 53, 5622-5632.

(44) Helms, J. R.; Stubbins, A.; Ritchie, J. D.; Minor, E. C.; Kieber, D. J.; Mopper, K. Absorption Spectral Slopes and Slope Ratios as Indicators of Molecular Weight, Source, and Photobleaching of Chromophoric Dissolved Organic Matter. Limnol. Oceanogr. 2008, 53, 955-969.

(45) Peuravuori, J.; Pihlaja, K. Molecular Size Distribution and Spectroscopic Properties of Aquatic Humic Substances. Anal. Chim. Acta 1997, 337, 133-149.

(46) Lester, Y.; Sharpless, C. M.; Mamane, H.; Linden, K. G. Production of Photo-Oxidants by Dissolved Organic Matter During UV Water Treatment. Environ. Sci. Technol. 2013, 47, 11726-11733.

(47) Haag, W. R.; Hoigné, J.; Gassman, E.; Braun, A. M. Singlet Oxygen in Surface Waters - Part II: Quantum Yields of Its Production by Some Natural Humic Materials as a Function of Wavelength. Chemosphere 1984, 13, 641-650.

(48) Dalrymple, R. M.; Carfagno, A. K.; Sharpless, C. M. Correlations between Dissolved Organic Matter Optical Properties and Quantum Yields of Singlet Oxygen and Hydrogen Peroxide. Environ. Sci. Technol. 2010, 44, 5824-5829.

(49) Nkhili, E.; Boguta, P.; Bejger, R.; Guyot, G.; Sokołowska, Z.; Richard, C. Photosensitizing Properties of Water-Extractable Organic Matter from Soils. Chemosphere 2014, 95, 317-323.

(50) Hong, J.; Liu, J.; Wang, L.; Kong, S.; Tong, C.; Qin, J.; Chen, L.; Sui, Y.; Li, B. Characterization of Reactive Photoinduced Species in Rainwater. Environ. Sci. Pollut. Res. 2018, 25, 36368-36380.

(51) Li, M.; Bao, F.; Zhang, Y.; Sheng, H.; Chen, C.; Zhao, J. Photochemical Aging of Soot in the Aqueous Phase: Release of Dissolved Black Carbon and the Formation of ${ }^{1} \mathrm{O}_{2}$. Environ. Sci. Technol. 2019, 53, 12311-12319.

(52) Zhao, J.; Zhou, Y.; Li, C.; Xie, Q.; Chen, J.; Chen, G.; Peijnenburg, W. J. G. M.; Zhang, Y.; Qu, J. Development of a Quantitative Structure-Activity Relationship Model for Mechanistic Interpretation and Quantum Yield Prediction of Singlet Oxygen Generation from Dissolved Organic Matter. Sci. Total Environ. 2020, $712,136450$.

(53) Zhou, C.; Xie, Q.; Wang, J.; Chen, X.; Niu, J.; Chen, J. Effects of Dissolved Organic Matter Derived from Freshwater and Seawater on Photodegradation of Three Antiviral Drugs. Environ. Pollut. 2020, 258, 113700 .

(54) Glover, C. M.; Rosario-Ortiz, F. L. Impact of Halides on the Photoproduction of Reactive Intermediates from Organic Matter. Environ. Sci. Technol. 2013, 47, 13949-13956.

(55) Mostafa, S.; Rosario-Ortiz, F. L. Singlet Oxygen Formation from Wastewater Organic Matter. Environ. Sci. Technol. 2013, 47, 8179-8186.

(56) Cawley, K. M.; Korak, J. A.; Rosario-Ortiz, F. L. Quantum Yields for the Formation of Reactive Intermediates from Dissolved Organic Matter Samples from the Suwannee River. Environ. Eng. Sci. 2015, 32, 31-37.

(57) Mostafa, S.; Korak, J. A.; Shimabuku, K.; Glover, C. M.; Rosario-Ortiz, F. L. Relation between Optical Properties and Formation of Reactive Intermediates from Different Size Fractions of Organic Matter. In Advances in the Physicochemical Characterization of Dissolved Organic Matter: Impact on Natural and Engineered Systems; ACS Symposium Series; American Chemical Society, 2014; pp 159179.

(58) Zhang, D.; Yan, S.; Song, W. Photochemically Induced Formation of Reactive Oxygen Species (ROS) from Effluent Organic Matter. Environ. Sci. Technol. 2014, 48, 12645-12653.

(59) Fu, H.; Liu, H.; Mao, J.; Chu, W.; Li, Q.; Alvarez, P. J. J.; Qu, X.; Zhu, D. Photochemistry of Dissolved Black Carbon Released from Biochar: Reactive Oxygen Species Generation and Phototransformation. Environ. Sci. Technol. 2016, 50, 1218-1226.
(60) McKay, G.; Couch, K. D.; Mezyk, S. P.; Rosario-Ortiz, F. L. Investigation of the Coupled Effects of Molecular Weight and ChargeTransfer Interactions on the Optical and Photochemical Properties of Dissolved Organic Matter. Environ. Sci. Technol. 2016, 50, 80938102.

(61) Silva, M. P.; Lastre-Acosta, A. M.; Mostafa, S.; McKay, G.; Linden, K. G.; Rosario-Ortiz, F. L.; Teixeira, A. C. S. C. Photochemical Generation of Reactive Intermediates from UrbanWaste Bio-Organic Substances under UV and Solar Irradiation. Environ. Sci. Pollut. Res. 2017, 24, 18470-18478.

(62) Tenorio, R.; Fedders, A. C.; Strathmann, T. J.; Guest, J. S. Impact of Growth Phases on Photochemically Produced Reactive Species in the Extracellular Matrix of Algal Cultivation Systems. Environ. Sci. Water Res. Technol. 2017, 3, 1095-1108.

(63) Zhou, H.; Lian, L.; Yan, S.; Song, W. Insights into the PhotoInduced Formation of Reactive Intermediates from Effluent Organic Matter: The Role of Chemical Constituents. Water Res. 2017, 112, $120-128$.

(64) Chen, X.; Wang, J.; Chen, J.; Zhou, C.; Cui, F.; Sun, G. Photodegradation of 2-(2-Hydroxy-5-Methylphenyl)Benzotriazole (UV-P) in Coastal Seawaters: Important Role of DOM. J. Environ. Sci. 2019, 85, 129-137.

(65) Wang, J.; Chen, J.; Qiao, X.; Zhang, Y.; Uddin, M.; Guo, Z. Disparate Effects of DOM Extracted from Coastal Seawaters and Freshwaters on Photodegradation of 2,4-Dihydroxybenzophenone. Water Res. 2019, 151, 280-287.

(66) Wenk, J.; Nguyen, M. T.; Nelson, K. L. Natural Photosensitizers in Constructed Unit Process Wetlands: Photochemical Characterization and Inactivation of Pathogen Indicator Organisms. Environ. Sci. Technol. 2019, 53, 7724-7735.

(67) Baxter, R. M.; Carey, J. H. Reactions of Singlet Oxygen in Humic Waters. Freshwater Biol. 1982, 12, 285-292.

(68) Zepp, R. G.; Schlotzhauer, P. F.; Sink, R. M. Photosensitized Transformations Involving Electronic Energy Transfer in Natural Waters: Role of Humic Substances. Environ. Sci. Technol. 1985, 19, $74-81$.

(69) Aguer, J.-P.; Richard, C.; Andreux, F. Comparison of the Photoinductive Properties of Commercial, Synthetic and SoilExtracted Humic Substances. J. Photochem. Photobiol., A 1997, 103, $163-168$.

(70) Halladja, S.; ter Halle, A.; Aguer, J.-P.; Boulkamh, A.; Richard, C. Inhibition of Humic Substances Mediated Photooxygenation of Furfuryl Alcohol by 2,4,6-Trimethylphenol. Evidence for Reactivity of the Phenol with Humic Triplet Excited States. Environ. Sci. Technol. 2007, 41, 6066-6073.

(71) Sharpless, C. M. Lifetimes of Triplet Dissolved Natural Organic Matter (DOM) and the Effect of $\mathrm{NaBH}_{4}$ Reduction on Singlet Oxygen Quantum Yields: Implications for DOM Photophysics. Environ. Sci. Technol. 2012, 46, 4466-4473.

(72) Janssen, E. M.-L.; Erickson, P. R.; McNeill, K. Dual Roles of Dissolved Organic Matter as Sensitizer and Quencher in the Photooxidation of Tryptophan. Environ. Sci. Technol. 2014, 48, 4916-4924.

(73) Marchisio, A.; Minella, M.; Maurino, V.; Minero, C.; Vione, D. Photogeneration of Reactive Transient Species upon Irradiation of Natural Water Samples: Formation Quantum Yields in Different Spectral Intervals, and Implications for the Photochemistry of Surface Waters. Water Res. 2015, 73, 145-156.

(74) Maizel, A. C.; Remucal, C. K. Molecular Composition and Photochemical Reactivity of Size-Fractionated Dissolved Organic Matter. Environ. Sci. Technol. 2017, 51, 2113-2123.

(75) Maizel, A. C.; Remucal, C. K. The Effect of Probe Choice and Solution Conditions on the Apparent Photoreactivity of Dissolved Organic Matter. Environ. Sci. Process. Impacts 2017, 19, 1040-1050.

(76) Maizel, A. C.; Li, J.; Remucal, C. K. Relationships Between Dissolved Organic Matter Composition and Photochemistry in Lakes of Diverse Trophic Status. Environ. Sci. Technol. 2017, 51, 96249632. 
(77) Berg, S. M.; Whiting, Q. T.; Herrli, J. A.; Winkels, R.; Wammer, K. H.; Remucal, C. K. The Role of Dissolved Organic Matter Composition in Determining Photochemical Reactivity at the Molecular Level. Environ. Sci. Technol. 2019, 53, 11725-11734.

(78) O'Connor, M.; Helal, S. R.; Latch, D. E.; Arnold, W. A. Quantifying Photo-Production of Triplet Excited States and Singlet Oxygen from Effluent Organic Matter. Water Res. 2019, 156, $23-33$.

(79) Wan, D.; Sharma, V. K.; Liu, L.; Zuo, Y.; Chen, Y. Mechanistic Insight into the Effect of Metal Ions on Photogeneration of Reactive Species from Dissolved Organic Matter. Environ. Sci. Technol. 2019, 53, 5778-5786.

(80) Zhou, H.; Yan, S.; Lian, L.; Song, W. Triplet-State Photochemistry of Dissolved Organic Matter: Triplet-State Energy Distribution and Surface Electric Charge Conditions. Environ. Sci. Technol. 2019, 53, 2482-2490.

(81) Cory, R. M.; Cotner, J. B.; McNeill, K. Quantifying Interactions between Singlet Oxygen and Aquatic Fulvic Acids. Environ. Sci. Technol. 2009, 43, 718-723.

(82) Chu, C.; Lundeen, R. A.; Sander, M.; McNeill, K. Assessing the Indirect Photochemical Transformation of Dissolved Combined Amino Acids through the Use of Systematically Designed HistidineContaining Oligopeptides. Environ. Sci. Technol. 2015, 49, 1279812807.

(83) Paul, A.; Hackbarth, S.; Vogt, R. D.; Röder, B.; Burnison, B. K.; Steinberg, C. E. W. Photogeneration of Singlet Oxygen by Humic Substances: Comparison of Humic Substances of Aquatic and Terrestrial Origin. Photochem. Photobiol. Sci. 2004, 3, 273-280.

(84) Carlos, L.; Cipollone, M.; Soria, D. B.; Sergio Moreno, M.; Ogilby, P. R.; García Einschlag, F. S.; Mártire, D. O. The Effect of Humic Acid Binding to Magnetite Nanoparticles on the Photogeneration of Reactive Oxygen Species. Sep. Purif. Technol. 2012, 91, 23-29.

(85) Pozdnyakov, I. P.; Salomatova, V. A.; Parkhats, M. V.; Dzhagarov, B. M.; Bazhin, N. M. Efficiency of Singlet Oxygen Generation by Fulvic Acids and Its Influence on UV Photodegradation of Herbicide Amitrole in Aqueous Solutions. Mendeleev Commun. 2017, 27, 399-401.

(86) Bilski, P.; Burkhart, J. G.; Chignell, C. F. Photochemical Characterization of Water Samples from Minnesota and Vermont Sites with Malformed Frogs: Potential Influence of Photosensitization by Singlet Molecular Oxygen $\left({ }^{1} \mathrm{O}_{2}\right)$ and Free Radicals on Aquatic Toxicity. Aquat. Toxicol. 2003, 65, 229-241.

(87) Wilkinson, F.; Helman, W. P.; Ross, A. B. Rate Constants for the Decay and Reactions of the Lowest Electronically Excited Singlet State of Molecular Oxygen in Solution. An Expanded and Revised Compilation. J. Phys. Chem. Ref. Data 1995, 24, 663-677.

(88) Turro, N. J.; Ramamurthy, V.; Scaiano, J. C. Modern Molecular Photochemistry of Organic Molecules; University Science Books: Sausalito, CA, 2010.

(89) Schweitzer, C.; Schmidt, R. Physical Mechanisms of Generation and Deactivation of Singlet Oxygen. Chem. Rev. 2003, 103, 16851758.

(90) Ogilby, P. R. Singlet Oxygen: There Is Indeed Something New under the Sun. Chem. Soc. Rev. 2010, 39, 3181-3209.

(91) Nosaka, Y.; Nosaka, A. Y. Generation and Detection of Reactive Oxygen Species in Photocatalysis. Chem. Rev. 2017, 117, 11302-11336.

(92) Nonell, S.; Flors, C. Singlet Oxygen: Applications in Biosciences and Nanosciences; The Royal Society of Chemistry, 2016; Vol. 1.

(93) Nonell, S.; Flors, C. Singlet Oxygen: Applications in Biosciences and Nanosciences; The Royal Society of Chemistry, 2016; Vol. 2.

(94) Boyle, E. S.; Guerriero, N.; Thiallet, A.; Vecchio, R. D.; Blough, N. V. Optical Properties of Humic Substances and CDOM: Relation to Structure. Environ. Sci. Technol. 2009, 43, 2262-2268.

(95) Schmitt, M.; Erickson, P. R.; McNeill, K. Triplet-State Dissolved Organic Matter Quantum Yields and Lifetimes from Direct Observation of Aromatic Amine Oxidation. Environ. Sci. Technol. 2017, 51, 13151-13160.
(96) Wetzel, R. G. Oxygen. In Limnology, 3rd ed.; Academic Press: San Diego, 2001; pp 151-168.

(97) Paterson, M. J.; Christiansen, O.; Jensen, F.; Ogilby, P. R. Overview of Theoretical and Computational Methods Applied to the Oxygen-Organic Molecule Photosystem. Photochem. Photobiol. 2006, $82,1136-1160$.

(98) Wilkinson, F.; Helman, W. P.; Ross, A. B. Quantum Yields for the Photosensitized Formation of the Lowest Electronically Excited Singlet State of Molecular Oxygen in Solution. J. Phys. Chem. Ref. Data 1993, 22, 113-262.

(99) Cabrerizo, F. M.; Arnbjerg, J.; Denofrio, M. P.; Erra-Balsells, R.; Ogilby, P. R. Fluorescence Quenching by Oxygen: "Debunking" a Classic Rule. ChemPhysChem 2010, 11, 796-798.

(100) Bregnhøj, M.; Blázquez-Castro, A.; Westberg, M.; Breitenbach, T.; Ogilby, P. R. Direct $765 \mathrm{Nm}$ Optical Excitation of Molecular Oxygen in Solution and in Single Mammalian Cells. J. Phys. Chem. B 2015, 119, 5422-5429.

(101) Bregnhøj, M.; Westberg, M.; Jensen, F.; Ogilby, P. R. SolventDependent Singlet Oxygen Lifetimes: Temperature Effects Implicate Tunneling and Charge-Transfer Interactions. Phys. Chem. Chem. Phys. 2016, 18, 22946-22961.

(102) Davis, C. A.; McNeill, K.; Janssen, E. M.-L. Non-Singlet Oxygen Kinetic Solvent Isotope Effects in Aquatic Photochemistry. Environ. Sci. Technol. 2018, 52, 9908-9916.

(103) Appiani, E.; Ossola, R.; Latch, D. E.; Erickson, P. R.; McNeill, K. Aqueous Singlet Oxygen Reaction Kinetics of Furfuryl Alcohol: Effect of Temperature, $\mathrm{pH}$, and Salt Content. Environ. Sci. Process. Impacts 2017, 19, 507-516.

(104) Clennan, E. L. Overview of the Chemical Reactions of Singlet Oxygen. In Singlet Oxygen: Applications in Biosciences and Nanosciences; The Royal Society of Chemistry, 2016; Vol. 1, pp 351-367.

(105) Ouannes, C.; Wilson, T. Quenching of Singlet Oxygen by Tertiary Aliphatic Amines. Effect of DABCO (1,4Diazabicyclo[2.2.2] Octane). J. Am. Chem. Soc. 1968, 90, 6527-6528.

(106) Bregnhøj, M.; Westberg, M.; Minaev, B. F.; Ogilby, P. R. Singlet Oxygen Photophysics in Liquid Solvents: Converging on a Unified Picture. Acc. Chem. Res. 2017, 50, 1920-1927.

(107) Thorning, F.; Jensen, F.; Ogilby, P. R. Modeling the Effect of Solvents on Nonradiative Singlet Oxygen Deactivation: Going beyond Weak Coupling in Intermolecular Electronic-to-Vibrational Energy Transfer. J. Phys. Chem. B 2020, 124, 2245-2254.

(108) Krasnovsky, A. A., Jr. Photonics of Molecular Oxygen in Aqueous Solutions. Phys. Wave Phenom. 2020, 28, 116-134.

(109) Jiménez-Banzo, A.; Ragàs, X.; Kapusta, P.; Nonell, S. TimeResolved Methods in Biophysics. 7. Photon Counting vs. Analog Time-Resolved Singlet Oxygen Phosphorescence Detection. Photochem. Photobiol. Sci. 2008, 7, 1003-1010.

(110) Aguer, J. P.; Richard, C. Use of Furoin as Probe Molecule for the Involvement of Singlet Oxygen in Aqueous Phase Photoinduced Transformations. Toxicol. Environ. Chem. 1993, 39, 217-227.

(111) Ogawa, S.; Fukui, S.; Hanasaki, Y.; Asano, K.; Uegaki, H.; Sumiko, F.; Ryosuke, S. Determination Method of Singlet Oxygen in the Atmosphere by Use of $\alpha$-Terpinene. Chemosphere 1991, 22, $1211-1225$

(112) Gomes, A.; Fernandes, E.; Lima, J. L. F. C. Fluorescence Probes Used for Detection of Reactive Oxygen Species. J. Biochem. Biophys. Methods 2005, 65, 45-80.

(113) You, Y. Chemical Tools for the Generation and Detection of Singlet Oxygen. Org. Biomol. Chem. 2018, 16, 4044-4060.

(114) Hideg, É. A Comparative Study of Fluorescent Singlet Oxygen Probes in Plant Leaves. Open Life Sci. 2008, 3, 273-284.

(115) Flors, C.; Fryer, M. J.; Waring, J.; Reeder, B.; Bechtold, U.; Mullineaux, P. M.; Nonell, S.; Wilson, M. T.; Baker, N. R. Imaging the Production of Singlet Oxygen in Vivo Using a New Fluorescent Sensor, Singlet Oxygen Sensor Green ${ }^{\circledR}$. J. Exp. Bot. 2006, 57, 17251734.

(116) Koh, E.; Fluhr, R. Singlet Oxygen Detection in Biological Systems: Uses and Limitations. Plant Signaling Behav. 2016, 11, No. e1192742. 
(117) Lemp, E.; Zanocco, A. L. Singlet Oxygen Chemical Acceptors. In Singlet Oxygen: Applications and Biosciences and Nanosciences; The Royal Society of Chemistry, 2016; Vol. 2, pp 83-101.

(118) Haag, W. R.; Hoigné, J. Singlet Oxygen in Surface Waters. 3. Photochemical Formation and Steady-State Concentrations in Various Types of Waters. Environ. Sci. Technol. 1986, 20, 341-348.

(119) Nonell, S.; Flors, C. Steady-State and Time-Resolved Singlet Oxygen Phosphorescence Detection in the Near-IR. In Singlet Oxygen: Applications in Biosciences and Nanosciences; The Royal Society of Chemistry, 2016; Vol. 2, pp 7-26.

(120) Boso, G.; Ke, D.; Korzh, B.; Bouilloux, J.; Lange, N.; Zbinden, H. Time-Resolved Singlet-Oxygen Luminescence Detection with an Efficient and Practical Semiconductor Single-Photon Detector. Biomed. Opt. Express 2016, 7, 211-224.

(121) Parker, J. G.; Stanbro, W. D. Dependence of Photosensitized Singlet Oxygen Production on Porphyrin Structure and Solvent. Prog. Clin. Biol. Res. 1984, 170, 259-284.

(122) Braslavsky, S. E. Glossary of Terms Used in Photochemistry, Third Edition (IUPAC Recommendations 2006). Pure Appl. Chem. 2007, 79, 293-465.

(123) Verhoeven, J. W. Glossary of Terms Used in Photochemistry (IUPAC Recommendations 1996). Pure Appl. Chem. 1996, 68, 22232286.

(124) Shaw, H.; Toby, S. Light Absorption in Photochemistry. J. Chem. Educ. 1966, 43, 408-410.

(125) Björn, L. O. The Measurement of Light. In Photobiology: The Science of Light and Life; Björn, L. O., Ed.; Springer: New York, 2015; pp 37-49.

(126) Braun, A. M.; Maurette, M.-T.; Oliveros, E. Radiometry and Actinometry. In Photochemical Technology; John Wiley \& Sons: Chichester, UK, 1991; pp 51-106.

(127) Bolton, J. R.; Linden, K. G. Standardization of Methods for Fluence (UV Dose) Determination in Bench-Scale UV Experiments. J. Environ. Eng. 2003, 129, 209-215.

(128) Dulin, D.; Mill, T. Development and Evaluation of Sunlight Actinometers. Environ. Sci. Technol. 1982, 16, 815-820.

(129) Asenath-Smith, E.; Ambrogi, E. K.; Moores, L. C.; Newman, S. D.; Brame, J. A. Leveraging Chemical Actinometry and Optical Radiometry to Reduce Uncertainty in Photochemical Research. J. Photochem. Photobiol., A 2019, 372, 279-287.

(130) Laszakovits, J. R.; Berg, S. M.; Anderson, B. G.; O’Brien, J. E.; Wammer, K. H.; Sharpless, C. M. $p$-Nitroanisole/Pyridine and $p$ Nitroacetophenone/Pyridine Actinometers Revisited: Quantum Yield in Comparison to Ferrioxalate. Environ. Sci. Technol. Lett. 2017, 4, $11-14$.

(131) Leifer, A. Kinetics of Environmental Aquatic Photochemistry; American Chemical Society, WA, 1988.

(132) Schwarzenbach, R. P.; Gschwend, P. M.; Imboden, D. M. Direct Photolysis. In Environmental Organic Chemistry; John Wiley \& Sons, Inc., 2002; pp 611-654.

(133) Hatchard, C. G.; Parker, C. A.; Bowen, E. J. A New Sensitive Chemical Actinometer - II. Potassium Ferrioxalate as a Standard Chemical Actinometer. Proc. R. Soc. London Ser. Math. Phys. Sci. 1956, 235, 518-536.

(134) Galbavy, E. S.; Ram, K.; Anastasio, C. 2-Nitrobenzaldehyde as a Chemical Actinometer for Solution and Ice Photochemistry. J. Photochem. Photobiol., A 2010, 209, 186-192.

(135) Apell, J. N.; McNeill, K. Updated and Validated Solar Irradiance Reference Spectra for Estimating Environmental Photodegradation Rates. Environ. Sci. Process. Impacts 2019, 21, 427-437.

(136) Armarego, W. L. F.; Chai, C. C. L. Purification of Organic Chemicals - Aromatic Compounds. In Purification of Laboratory Chemicals, 6th ed.; Butterworth-Heinemann, 2009; p 316.

(137) Morales, R. G. E.; Java, G. P.; Cabrera, S. Solar Ultraviolet Radiation Measurements by $o$-Nitrobenzaldehyde Actinometry. Limnol. Oceanogr. 1993, 38, 703-705.

(138) Kuhn, H. J.; Braslavsky, S. E.; Schmidt, R. Chemical Actinometry (IUPAC Technical Report). Pure Appl. Chem. 2004, $76,2105-2146$.
(139) Kirk, A. D.; Namasivayam, C. Errors in Ferrioxalate Actinometry. Anal. Chem. 1983, 55, 2428-2429.

(140) Bowman, W. D.; Demas, J. N. Ferrioxalate Actinometry. A Warning on Its Correct Use. J. Phys. Chem. 1976, 80, 2434-2435.

(141) Leifer, A.. Part II.B. Direct Photoreaction Test Methods Laboratory Determination of the Direct Photoreaction Quantum Yield in Aqueous Solution and Sunlight Photoreaction Rates. In Kinetics of Environmental Aquatic Photochemistry; American Chemical Society, Washington, DC, 1988; pp 139-169.

(142) Goldstein, S.; Rabani, J. The Ferrioxalate and Iodide-Iodate Actinometers in the UV Region. J. Photochem. Photobiol., A 2008, 193, $50-55$.

(143) Wardle, B. Light Absorption and Electronically-Excited States. In Principles and Applications of Photochemistry; John Wiley \& Sons: Chichester, UK, 2009; pp 29-46.

(144) Hu, C.; Muller-Karger, F. E.; Zepp, R. G. Absorbance, Absorption Coefficient, and Apparent Quantum Yield: A Comment on Common Ambiguity in the Use of These Optical Concepts. Limnol. Oceanogr. 2002, 47, 1261-1267.

(145) Apell, J. N.; Pflug, N. C.; McNeill, K. Photodegradation of Fludioxonil and Other Pyrroles: The Importance of Indirect Photodegradation for Understanding Environmental Fate and Photoproduct Formation. Environ. Sci. Technol. 2019, 53, 11240-11250.

(146) Ruiz, A. E.; Caregnato, P.; Arce, V. B.; Schiavoni, M. d. 1. M.; Mora, V. C.; Gonzalez, M. C.; Allegretti, P. E.; Mártire, D. O. Synthesis and Characterization of Butoxylated Silica Nanoparticles. Reaction with Benzophenone Triplet States. J. Phys. Chem. C 2007, $111,7623-7628$.

(147) Eckhoff, D. A.; Sutin, J. D. B.; Clegg, R. M.; Gratton, E.; Rogozhina, E. V.; Braun, P. V. Optical Characterization of Ultrasmall $\mathrm{Si}$ Nanoparticles Prepared through Electrochemical Dispersion of Bulk Si. J. Phys. Chem. B 2005, 109, 19786-19797.

(148) Milazzo, G.; Caroli, S.; Palumbo-Doretti, M.; Violante, N. Reference Materials for Ultraviolet Spectrophotometry. Anal. Chem. 1977, 49, 711-717.

(149) National Institute of Standards and Technology. NIST Chemistry WebBook https://webbook.nist.gov/chemistry/ (accessed Oct 19, 2020).

(150) Science-softCon. UV/Vis ${ }^{+}$Photochemistry Database https:// science-softcon.de/spectra/ (accessed Oct 19, 2020).

(151) Noelle, A.; Vandaele, A. C.; Martin-Torres, J.; Yuan, C.; Rajasekhar, B. N.; Fahr, A.; Hartmann, G. K.; Lary, D.; Lee, Y.-P.; Limão-Vieira, P.; Locht, R.; McNeill, K.; Orlando, J. J.; Salama, F.; Wayne, R. P. UV/Vis ${ }^{+}$Photochemistry Database: Structure, Content and Applications. J. Quant. Spectrosc. Radiat. Transfer 2020, 253, 107056.

(152) Segado, M.; Reguero, M. Mechanism of the Photochemical Process of Singlet Oxygen Production by Phenalenone. Phys. Chem. Chem. Phys. 2011, 13, 4138-4148.

(153) Nardello, V.; Azaroual, N.; Cervoise, I.; Vermeersch, G.; Aubry, J.-M. Synthesis and Photooxidation of Sodium 1,3-Cyclohexadiene-1,4-Diethanoate: A New Colorless and Water-Soluble Trap of Singlet Oxygen. Tetrahedron 1996, 52, 2031-2046.

(154) Haag, W. R.; Hoigné, J.; Gassman, E.; Braun, A. M. Singlet Oxygen in Surface Waters - Part I: Furfuryl Alcohol as a Trapping Agent. Chemosphere 1984, 13, 631-640.

(155) Rosario-Ortiz, F. L.; Canonica, S. Probe Compounds to Assess the Photochemical Activity of Dissolved Organic Matter. Environ. Sci. Technol. 2016, 50, 12532-12547.

(156) Latch, D. E.; Stender, B. L.; Packer, J. L.; Arnold, W. A.; McNeill, K. Photochemical Fate of Pharmaceuticals in the Environment: Cimetidine and Ranitidine. Environ. Sci. Technol. 2003, 37, 3342-3350.

(157) Meek, S. J.; Pitman, C. L.; Miller, A. J. M. Deducing Reaction Mechanism: A Guide for Students, Researchers, and Instructors. J. Chem. Educ. 2016, 93, 275-286.

(158) Iroegbu, A. O.; Hlangothi, S. P. Effects of the Type of Catalyst on the Polymerisation Mechanism of Furfuryl Alcohol and Its Resultant Properties. Chem. Afr. 2018, 1, 187-197. 
(159) Iroegbu, A. O.; Hlangothi, S. P. Furfuryl Alcohol a Versatile, Eco-Sustainable Compound in Perspective. Chem. Afr. 2019, 2, 223239.

(160) Maddigapu, P. R.; Bedini, A.; Minero, C.; Maurino, V.; Vione, D.; Brigante, M.; Mailhot, G.; Sarakha, M. The pH-Dependent Photochemistry of Anthraquinone-2-Sulfonate. Photochem. Photobiol. Sci. 2010, 9, 323-330.

(161) al Housari, F.; Vione, D.; Chiron, S.; Barbati, S. Reactive Photoinduced Species in Estuarine Waters. Characterization of Hydroxyl Radical, Singlet Oxygen and Dissolved Organic Matter Triplet State in Natural Oxidation Processes. Photochem. Photobiol. Sci. 2010, 9, 78-86.

(162) Haag, W. R.; Mill, T. Rate Constants for Interaction if ${ }^{1} \mathrm{O}_{2}\left({ }^{1} \Delta_{\mathrm{g}}\right)$ with Azide Ion in Water. Photochem. Photobiol. 1987, 45, 317-321.

(163) Schmidt, R.; Tanielian, C.; Dunsbach, R.; Wolff, C. Phenalenone, a Universal Reference Compound for the Determination of Quantum Yields of Singlet Oxygen $\mathrm{O}_{2}\left({ }^{1} \Delta_{\mathrm{g}}\right)$ Sensitization. J. Photochem. Photobiol., A 1994, 79, 11-17.

(164) Gandin, E.; Lion, Y.; Van de Vorst, A. Quantum Yield of Singlet Oxygen Production by Xanthene Derivatives. Photochem. Photobiol. 1983, 37, 271-278.

(165) Fresnadillo, D. G.; Lacombe, S. Reference Photosensitizers for the Production of Singlet Oxygen. In Singlet Oxygen: Applications in Biosciences and Nanosciences 2016, 1, 105-143.

(166) Phenalenone, Chem Spider - Properties - Predicted EPISuite http://www.chemspider.com/Chemical-Structure.10582.html (accessed October 10, 2019).

(167) Späth, A.; Leibl, C.; Cieplik, F.; Lehner, K.; Regensburger, J.; Hiller, K.-A.; Bäumler, W.; Schmalz, G.; Maisch, T. Improving Photodynamic Inactivation of Bacteria in Dentistry: Highly Effective and Fast Killing of Oral Key Pathogens with Novel Tooth-Colored Type-II Photosensitizers. J. Med. Chem. 2014, 57, 5157-5168.

(168) Martí, C.; Jürgens, O.; Cuenca, O.; Casals, M.; Nonell, S. Aromatic Ketones as Standards for Singlet Molecular Oxygen $\mathrm{O}_{2}\left({ }^{1} \Delta_{\mathrm{g}}\right)$ Photosensitization. Time-Resolved Photoacoustic and nearIR Emission Studies. J. Photochem. Photobiol., A 1996, 97, 11-18.

(169) Redmond, R. W.; Gamlin, J. N. A Compilation of Singlet Oxygen Yields from Biologically Relevant Molecules. Photochem. Photobiol. 1999, 70, 391-475.

(170) Flors, C.; Ogilby, P. R.; Luis, J. G.; Grillo, T. A.; Izquierdo, L. R.; Gentili, P.-L.; Bussotti, L.; Nonell, S. Phototoxic Phytoalexins. Processes That Compete with the Photosensitized Production of Singlet Oxygen by 9-Phenylphenalenones. Photochem. Photobiol. 2006, 82, 95-103.

(171) Cooke, R. G.; Edwards, J. M. Naturally Occurring Phenalenones and Related Compounds. In Fortschritte der Chemie organischer Naturstoffe/Progress in the Chemistry of Organic Natural Products; Springer: Vienna, 1981; pp 153-190.

(172) International Humic Substances Society https://ihss. humicsubstances.org/ (accessed Jun 2, 2020).

(173) Rodgers, M. A. J.; Snowden, P. T. Lifetime of Oxygen $\left(\mathrm{O}_{2}\left({ }^{1} \Delta_{\mathrm{g}}\right)\right)$ in Liquid Water as Determined by Time-Resolved Infrared Luminescence Measurements. J. Am. Chem. Soc. 1982, 104, 55415543.

(174) Rodgers, M. A. J. Solvent-Induced Deactivation of Singlet Oxygen: Additivity Relationships in Nonaromatic Solvents. J. Am. Chem. Soc. 1983, 105, 6201-6205.

(175) Jensen, R. L.; Arnbjerg, J.; Ogilby, P. R. Temperature Effects on the Solvent-Dependent Deactivation of Singlet Oxygen. J. Am. Chem. Soc. 2010, 132, 8098-8105.

(176) WebPlot Digitizer - Copyright 2010-2019 Ankit Rohatgi https://apps.automeris.io/wpd/ (accessed May 11, 2020).

(177) Source Materials for IHSS Samples. IHSS. http://humicsubstances.org/source-materials-for-ihss-samples/ (accessed February 2, 2020).

(178) Brown, A.; McKnight, D. M.; Chin, Y.-P.; Roberts, E. C.; Uhle, M. Chemical Characterization of Dissolved Organic Material in
Pony Lake, a Saline Coastal Pond in Antarctica. Mar. Chem. 2004, 89, 327-337.

(179) Averett, R. C.; Leenheer, J. A.; McKnight, D. M.; Thorn, K. A. Humic Substances in the Suwannee River, Georgia; Interactions, Properties, and Proposed Structures; Water Supply Paper; USGS Numbered Series 2373; U.S. G.P.O.; U.S. Geological Survey, Map Distribution, 1994.

(180) Fimmen, R. L.; Cory, R. M.; Chin, Y.-P.; Trouts, T. D.; McKnight, D. M. Probing the Oxidation-Reduction Properties of Terrestrially and Microbially Derived Dissolved Organic Matter. Geochim. Cosmochim. Acta 2007, 71, 3003-3015.

(181) Olk, D. C.; Bloom, P. R.; Perdue, E. M.; McKnight, D. M.; Chen, Y.; Farenhorst, A.; Senesi, N.; Chin, Y.-P.; Schmitt-Kopplin, P.; Hertkorn, N.; Harir, M. Environmental and Agricultural Relevance of Humic Fractions Extracted by Alkali from Soils and Natural Waters. J. Environ. Qual. 2019, 48, 217-232.

(182) Lehmann, J.; Kleber, M. The Contentious Nature of Soil Organic Matter. Nature 2015, 528, 60-68.

(183) Kleber, M.; Lehmann, J. Humic Substances Extracted by Alkali Are Invalid Proxies for the Dynamics and Functions of Organic Matter in Terrestrial and Aquatic Ecosystems. J. Environ. Qual. 2019, 48, 207-216.

(184) Malcolm, R. L.; MacCarthy, P. Limitations in the Use of Commercial Humic Acids in Water and Soil Research. Environ. Sci. Technol. 1986, 20, 904-911.

(185) Esteves, V. I.; Otero, M.; Duarte, A. C. Comparative Characterization of Humic Substances from the Open Ocean, Estuarine Water and Fresh Water. Org. Geochem. 2009, 40, 942-950.

(186) Parker, K. M.; Mitch, W. A. Halogen Radicals Contribute to Photooxidation in Coastal and Estuarine Waters. Proc. Natl. Acad. Sci. U. S. A. 2016, 113, 5868-5873.

(187) Baumgardner, R. E.; Kronmiller, K. G.; Anderson, J. B.; Bowser, J. J.; Edgerton, E. S. Development of an Automated Cloud Water Collection System for Use in Atmospheric Monitoring Networks. Atmos. Environ. 1997, 31, 2003-2010.

(188) Jaffé, R.; Ding, Y.; Niggemann, J.; Vähätalo, A. V.; Stubbins, A.; Spencer, R. G. M.; Campbell, J.; Dittmar, T. Global Charcoal Mobilization from Soils via Dissolution and Riverine Transport to the Oceans. Science 2013, 340, 345-347.

(189) Wagner, S.; Jaffé, R.; Stubbins, A. Dissolved Black Carbon in Aquatic Ecosystems. Limnol. Oceanogr. Lett. 2018, 3, 168-185.

(190) Stubbins, A.; Niggemann, J.; Dittmar, T. Photo-Lability of Deep Ocean Dissolved Black Carbon. Biogeosciences 2012, 9, 16611670.

(191) Henderson, R. K.; Baker, A.; Parsons, S. A.; Jefferson, B. Characterisation of Algogenic Organic Matter Extracted from Cyanobacteria, Green Algae and Diatoms. Water Res. 2008, 42, 3435-3445.

(192) Pivokonsky, M.; Safarikova, J.; Baresova, M.; Pivokonska, L.; Kopecka, I. A Comparison of the Character of Algal Extracellular versus Cellular Organic Matter Produced by Cyanobacterium, Diatom and Green Alga. Water Res. 2014, 51, 37-46.

(193) Malecha, K. T.; Nizkorodov, S. A. Photodegradation of Secondary Organic Aerosol Particles as a Source of Small, Oxygenated Volatile Organic Compounds. Environ. Sci. Technol. 2016, 50, 99909997.

(194) Aguer, J. P.; Richard, C. Photochemical Behaviour of Humic Acid Synthesized from Phenol. J. Photochem. Photobiol., A 1994, 84, 69-73.

(195) Appiani, E.; McNeill, K. Photochemical Production of Singlet Oxygen from Particulate Organic Matter. Environ. Sci. Technol. 2015, 49, 3514-3522.

(196) Latch, D. E.; McNeill, K. Microheterogeneity of Singlet Oxygen Distributions in Irradiated Humic Acid Solutions. Science 2006, 311, 1743-1747.

(197) Aiken, G. R.; McKnight, D. M.; Thorn, K. A.; Thurman, E. M. Isolation of Hydrophilic Organic Acids from Water Using Nonionic Macroporous Resins. Org. Geochem. 1992, 18, 567-573. 
(198) Spencer, R. G. M.; Aiken, G. R.; Dyda, R. Y.; Butler, K. D.; Bergamaschi, B. A.; Hernes, P. J. Comparison of XAD with Other Dissolved Lignin Isolation Techniques and a Compilation of Analytical Improvements for the Analysis of Lignin in Aquatic Settings. Org. Geochem. 2010, 41, 445-453.

(199) Isolation of IHSS Samples. IHSS. http://humic-substances. org/isolation-of-ihss-samples/ (accessed February 2, 2020).

(200) Sun, L.; Perdue, E. M.; McCarthy, J. F. Using Reverse Osmosis to Obtain Organic Matter from Surface and Ground Waters. Water Res. 1995, 29, 1471-1477.

(201) Collection of IHSS Samples. IHSS. http://humic-substances. org/collection-of-ihss-samples/ (accessed February 25, 2020).

(202) Repeta, D. J. Chemical Characterization and Cycling of Dissolved Organic Matter. In Biogeochemistry of Marine Dissolved Organic Matter, 2nd ed.; Academic Press: Boston, 2015; pp 21-63.

(203) Qu, X.; Fu, H.; Mao, J.; Ran, Y.; Zhang, D.; Zhu, D. Chemical and Structural Properties of Dissolved Black Carbon Released from Biochars. Carbon 2016, 96, 759-767.

(204) Roper, C.; Delgado, L. S.; Barrett, D.; Massey Simonich, S. L.; Tanguay, R. L. $\mathrm{PM}_{2.5}$ Filter Extraction Methods: Implications for Chemical and Toxicological Analyses. Environ. Sci. Technol. 2019, 53, 434-442.

(205) Miljevic, B.; Hedayat, F.; Stevanovic, S.; Fairfull-Smith, K. E.; Bottle, S. E.; Ristovski, Z. D. To Sonicate or Not to Sonicate PM Filters: Reactive Oxygen Species Generation Upon Ultrasonic Irradiation. Aerosol Sci. Technol. 2014, 48, 1276-1284.

(206) Bein, K. J.; Wexler, A. S. A High-Efficiency, Low-Bias Method for Extracting Particulate Matter from Filter and Impactor Substrates. Atmos. Environ. 2014, 90, 87-95.

(207) Bein, K. J.; Wexler, A. S. Compositional Variance in Extracted Particulate Matter Using Different Filter Extraction Techniques. Atmos. Environ. 2015, 107, 24-34.

(208) Yu, K.-P.; Chen, Y.-P.; Gong, J.-Y.; Chen, Y.-C.; Cheng, C.-C. Improving the Collection Efficiency of the Liquid Impinger for Ultrafine Particles and Viral Aerosols by Applying Granular Bed Filtration. J. Aerosol Sci. 2016, 101, 133-143.

(209) Haig, C. W.; Mackay, W. G.; Walker, J. T.; Williams, C. Bioaerosol Sampling: Sampling Mechanisms, Bioefficiency and Field Studies. J. Hosp. Infect. 2016, 93, 242-255.

(210) Bregnhøj, M.; Rodal-Cedeira, S.; Pastoriza-Santos, I.; Ogilby, P. R. Light Scattering versus Plasmon Effects: Optical Transitions in Molecular Oxygen near a Metal Nanoparticle. J. Phys. Chem. C 2018, 122, 15625-15634.

(211) Dittmar, T.; Koch, B.; Hertkorn, N.; Kattner, G. A Simple and Efficient Method for the Solid-Phase Extraction of Dissolved Organic Matter (SPE-DOM) from Seawater. Limnol. Oceanogr.: Methods 2008, 6, 230-235.

(212) Minor, E. C.; Swenson, M. M.; Mattson, B. M.; Oyler, A. R. Structural Characterization of Dissolved Organic Matter: A Review of Current Techniques for Isolation and Analysis. Environ. Sci. Process. Impacts 2014, 16, 2064-2079.

(213) Raeke, J.; Lechtenfeld, O. J.; Wagner, M.; Herzsprung, P.; Reemtsma, T. Selectivity of Solid Phase Extraction of Freshwater Dissolved Organic Matter and Its Effect on Ultrahigh Resolution Mass Spectra. Environ. Sci. Process. Impacts 2016, 18, 918-927.

(214) Sharpless, C. M.; Blough, N. V. The Importance of ChargeTransfer Interactions in Determining Chromophoric Dissolved Organic Matter (CDOM) Optical and Photochemical Properties. Environ. Sci. Process. Impacts 2014, 16, 654-671.

(215) Zepp, R. G.; Baughman, G. L.; Schlotzhauer, P. F. Comparison of Photochemical Behavior of Various Humic Substances in Water: II. Photosensitized Oxygenations. Chemosphere 1981, 10, 119-126.

(216) Schendorf, T. M.; Del Vecchio, R.; Bianca, M.; Blough, N. V. Combined Effects of $\mathrm{PH}$ and Borohydride Reduction on Optical Properties of Humic Substances (HS): A Comparison of Optical Models. Environ. Sci. Technol. 2019, 53, 6310-6319.
(217) Cox, B. A. A Review of Dissolved Oxygen Modelling Techniques for Lowland Rivers. Sci. Total Environ. 2003, 314-316, 303-334.

(218) Williams, R. J.; White, C.; Harrow, M. L.; Neal, C. Temporal and Small-Scale Spatial Variations of Dissolved Oxygen in the Rivers Thames, Pang and Kennet, UK. Sci. Total Environ. 2000, 251-252, 497-510.

(219) Andrews, S. S.; Caron, S.; Zafiriou, O. C. Photochemical Oxygen Consumption in Marine Waters: A Major Sink for Colored Dissolved Organic Matter? Limnol. Oceanogr. 2000, 45, 267-277.

(220) Whitehead, P. G.; Wilby, R. L.; Battarbee, R. W.; Kernan, M.; Wade, A. J. A Review of the Potential Impacts of Climate Change on Surface Water Quality. Hydrol. Sci. J. 2009, 54, 101-123.

(221) Fondriest Environmental, Inc. Dissolved Oxygen. Fundamentals of Environmental Measurements. https://www.fondriest.com/ environmental-measurements/parameters/water-quality/dissolvedoxygen/ (accessed Mar 2, 2020).

(222) Erickson, P. R.; Moor, K. J.; Werner, J. J.; Latch, D. E.; Arnold, W. A.; McNeill, K. Singlet Oxygen Phosphorescence as a Probe for Triplet-State Dissolved Organic Matter Reactivity. Environ. Sci. Technol. 2018, 52, 9170-9178.

(223) Parker, K. M.; Pignatello, J. J.; Mitch, W. A. Influence of Ionic Strength on Triplet-State Natural Organic Matter Loss by Energy Transfer and Electron Transfer Pathways. Environ. Sci. Technol. 2013, 47, 10987-10994.

(224) McKay, G.; Korak, J. A.; Erickson, P. R.; Latch, D. E.; McNeill, K.; Rosario-Ortiz, F. L. The Case Against Charge Transfer Interactions in Dissolved Organic Matter Photophysics. Environ. Sci. Technol. 2018, 52, 406-414.

(225) Blough, N. V.; Del Vecchio, R. Comment on The Case Against Charge Transfer Interactions in Dissolved Organic Matter Photophysics. Environ. Sci. Technol. 2018, 52, 5512-5513.

(226) McKay, G.; Korak, J. A.; Erickson, P. R.; Latch, D. E.; McNeill, K.; Rosario-Ortiz, F. L. Response to Comment on The Case Against Charge Transfer Interactions in Dissolved Organic Matter Photophysics. Environ. Sci. Technol. 2018, 52, 5514-5516.

(227) Chin, Y.-P.; Aiken, G.; O’Loughlin, E. Molecular Weight, Polydispersity, and Spectroscopic Properties of Aquatic Humic Substances. Environ. Sci. Technol. 1994, 28, 1853-1858.

(228) Remucal, C. K.; Cory, R. M.; Sander, M.; McNeill, K. Low Molecular Weight Components in an Aquatic Humic Substance As Characterized by Membrane Dialysis and Orbitrap Mass Spectrometry. Environ. Sci. Technol. 2012, 46, 9350-9359.

(229) Aeschbacher, M.; Graf, C.; Schwarzenbach, R. P.; Sander, M. Antioxidant Properties of Humic Substances. Environ. Sci. Technol. 2012, 46, 4916-4925.

(230) Romera-Castillo, C.; Jaffé, R. Free Radical Scavenging (Antioxidant Activity) of Natural Dissolved Organic Matter. Mar. Chem. 2015, 177, 668-676.

(231) Derrien, M.; Yang, L.; Hur, J. Lipid Biomarkers and Spectroscopic Indices for Identifying Organic Matter Sources in Aquatic Environments: A Review. Water Res. 2017, 112, 58-71.

(232) Ma, J.; Del Vecchio, R.; Golanoski, K. S.; Boyle, E. S.; Blough, N. V. Optical Properties of Humic Substances and CDOM: Effects of Borohydride Reduction. Environ. Sci. Technol. 2010, 44, 5395-5402.

(233) Mvula, E.; von Sonntag, C. Ozonolysis of Phenols in Aqueous Solution. Org. Biomol. Chem. 2003, 1, 1749-1756.

(234) von Sonntag, C.; von Gunten, U. Aromatic Compounds. In Chemistry of Ozone in Water and Wastewater Treatment: From Basic Principles to Applications; IWA Publishing: 2012; pp 109-130.

(235) Önnby, L.; Salhi, E.; McKay, G.; Rosario-Ortiz, F. L.; von Gunten, U. Ozone and Chlorine Reactions with Dissolved Organic Matter - Assessment of Oxidant-Reactive Moieties by Optical Measurements and the Electron Donating Capacities. Water Res. 2018, 144, 64-75.

(236) Tentscher, P. R.; Bourgin, M.; von Gunten, U. Ozonation of Para-Substituted Phenolic Compounds Yields p-Benzoquinones, Other Cyclic $\alpha, \beta$-Unsaturated Ketones, and Substituted Catechols. Environ. Sci. Technol. 2018, 52, 4763-4773. 
(237) Cooper, W. J.; Zika, R. G.; Petasne, R. G.; Plane, J. M. C. Photochemical Formation of Hydrogen Peroxide in Natural Waters Exposed to Sunlight. Environ. Sci. Technol. 1988, 22, 1156-1160.

(238) O’Sullivan, D. W.; Neale, P. J.; Coffin, R. B.; Boyd, T. J.; Osburn, C. L. Photochemical Production of Hydrogen Peroxide and Methylhydroperoxide in Coastal Waters. Mar. Chem. 2005, 97, 1433.

(239) Qian, J.; Mopper, K.; Kieber, D. J. Photochemical Production of the Hydroxyl Radical in Antarctic Waters. Deep Sea Res., Part I 2001, 48, 741-759.

(240) Vaughan, P. P.; Blough, N. V. Photochemical Formation of Hydroxyl Radical by Constituents of Natural Waters. Environ. Sci. Technol. 1998, 32, 2947-2953.

(241) Zhu, Y.; Kieber, D. J. Concentrations and Photochemistry of Acetaldehyde, Glyoxal, and Methylglyoxal in the Northwest Atlantic Ocean. Environ. Sci. Technol. 2019, 53, 9512-9521.

(242) White, E. M.; Kieber, D. J.; Sherrard, J.; Miller, W. L.; Mopper, K. Carbon Dioxide and Carbon Monoxide Photoproduction Quantum Yields in the Delaware Estuary. Mar. Chem. 2010, 118, 1121.

(243) Xie, H.; Moore, R. M.; Miller, W. L. Photochemical Production of Carbon Disulphide in Seawater. J. Geophys. Res. Oceans 1998, 103, 5635-5644.

(244) Blough, N. V. Photochemistry in the Sea-Surface Microlayer. In The Sea Surface and Global Change; Cambridge University Press: Cambridge, UK, 1997; pp 383-424.

(245) Chen, M.; Maie, N.; Parish, K.; Jaffé, R. Spatial and Temporal Variability of Dissolved Organic Matter Quantity and Composition in an Oligotrophic Subtropical Coastal Wetland. Biogeochemistry 2013, $115,167-183$.

(246) Maie, N.; Yang, C.; Miyoshi, T.; Parish, K.; Jaffé, R. Chemical Characteristics of Dissolved Organic Matter in an Oligotrophic Subtropical Wetland/Estuarine. Limnol. Oceanogr. 2005, 50, 23-35.

(247) Maie, N.; Pisani, O.; Jaffé, R. Mangrove Tannins in Aquatic Ecosystems: Their Fate and Possible Influence on Dissolved Organic Carbon and Nitrogen Cycling. Limnol. Oceanogr. 2008, 53, 160-171.

(248) Murphree, S. S.; Mason, J. D.; Bean, T. G.; Perry, M. C. Rapid Aqueous Borohydride Reduction of Carbonyls Under Sealed-Tube Microwave Conditions. Synth. Commun. 2012, 42, 1979-1986.

(249) Zeynizadeh, B.; Setamdideh, D. Water as a Green Solvent for Fast and Efficient Reduction of Carbonyl Compounds with $\mathrm{NaBH}_{4}$ under Microwave Irradiation. J. Chin. Chem. Soc. 2005, 52, 11791184.

(250) Denis, C.; Laignel, B.; Plusquellec, D.; Le Marouille, J.-Y.; Botrel, A. Highly Regio- and Stereoselective Reductions of Carbonyl Compounds in Aqueous Glycosidic Media. Tetrahedron Lett. 1996, $37,53-56$.

(251) Schmitzer, A. R.; Franceschi, S.; Perez, E.; Rico-Lattes, I.; Lattes, A.; Thion, L.; Erard, M.; Vidal, C. Reactivity at the Interface of Chiral Amphiphilic Dendrimers. High Asymmetric Reduction by $\mathrm{NaBH}_{4}$ of Various Prochiral Ketones. J. Am. Chem. Soc. 2001, 123, 5956-5961.

(252) Brown, W. H.; Foote, C. S.; Iverson, B. L.; Anslyn, E. V.; Novak, B. M. Aldehydes and Ketones. In Organic Chemistry, 6th ed.; Mary Finch, 2012; pp 581-648.

(253) Schendorf, T. M.; Del Vecchio, R.; Koech, K.; Blough, N. V. A Standard Protocol for $\mathrm{NaBH}_{4}$ Reduction of CDOM and HS. Limnol. Oceanogr.: Methods 2016, 14, 414-423.

(254) Bell, C. F.; Gallagher, B. C.; Lott, K. A. K.; Short, E. L.; Walton, L. Boric Acid Complexes of Phenolic Acids. Polyhedron 1991, 10, 613-618.

(255) Wang, H.; Zhou, H.; Ma, J.; Nie, J.; Yan, S.; Song, W. Triplet Photochemistry of Dissolved Black Carbon and Its Effects on the Photochemical Formation of Reactive Oxygen Species. Environ. Sci. Technol. 2020, 54, 4903-4911.

(256) Palma, D.; Sleiman, M.; Voldoire, O.; Beauger, A.; Parlanti, E.; Richard, C. Study of the Dissolved Organic Matter (DOM) of the Auzon Cut-off Meander (Allier River, France) by Spectral and
Photoreactivity Approaches. Environ. Sci. Pollut. Res. 2020, 27, 26385-26394.

(257) Chen, Y.; Hozalski, R. M.; Olmanson, L. G.; Page, B. P.; Finlay, J. C.; Brezonik, P. L.; Arnold, W. A. Prediction of Photochemically Produced Reactive Intermediates in Surface Waters via Satellite Remote Sensing. Environ. Sci. Technol. 2020, 54, 66716681.

(258) Wasswa, J.; Driscoll, C. T.; Zeng, T. Photochemical Characterization of Surface Waters from Lakes in the Adirondack Region of New York. Environ. Sci. Technol. 2020, 54, 10654-10667.

(259) Wan, D.; Kong, Y.; Selvinsimpson, S.; Luo, F.; Chen, Y. Effect of $\mathrm{UV}_{254}$ Disinfection on the Photoformation of Reactive Species from Effluent Organic Matter of Wastewater Treatment Plant. Water Res. 2020, 185, 116301.

(260) Guo, Z.; Wang, J.; Chen, X.; Cui, F.; Wang, T.; Zhou, C.; Song, G.; Zhang, S.; Chen, J. Photochemistry of Dissolved Organic Matter Extracted from Coastal Seawater: Excited Triplet-States and Contents of Phenolic Moieties. Water Res. 2021, 188, 116568.

(261) Leresche, F.; Torres-Ruiz, J. A.; Kurtz, T.; Gunten von, U.; Rosario-Ortiz, F. L. Optical Properties and Photochemical Production of Hydroxyl Radical and Singlet Oxygen after Ozonation of Dissolved Organic Matter. Environ. Sci. Water Res. Technol. 2020. DOI: $10.1039 /$ DOEW00878H.

(262) Wang, Z.; Lv, J.; Zhang, S.; Christie, P.; Zhang, S. Interfacial Molecular Fractionation on Ferrihydrite Reduces the Photochemical Reactivity of Dissolved Organic Matter. Environ. Sci. Technol. 2021. DOI: 10.1021/acs.est.0c07132.

(263) Zhou, Y.; Cheng, F.; He, D.; Zhang, Y.; Qu, J.; Yang, X.; Chen, J.; Peijnenburg, W. J. G. M. Effect of UV/Chlorine Treatment on Photophysical and Photochemical Properties of Dissolved Organic Matter. Water Res. 2021, 192, 116857. 